\title{
Mimicking Noncanonical Oxidations with Redox-Neutral Photocatalysis
}

\author{
Zheng Huang and Jean-Philip Lumb* \\ Department of Chemistry, McGill University, Montreal, QC H3A OB8, Canada. \\ *Corresponding author. Email: jean-philip.lumb@mcgill.ca
}

\section{ABSTRACT}

Noncanonical oxygenases are a family of Fe-containing enzymes that catalyze oxidative radical cyclizations. Despite creating key structural features that often define a natural product's complexity, the mechanisms of these oxidations remain poorly understood and difficult to mimic. In this work, we show that noncanonical cyclizations from lignan biosynthesis can be recreated when presumed biosynthetic radicals are generated using photocatalysis. These conditions afford the ensuing electron rich radicals sufficient time to undergo challenging 5- or 11-membered ring formation that create the defining structural features of the highly oxidized lignans taiwankadsurins $A, B$ and kadsuphilin N. By showing that these cyclizations can occur without enzymatic assistance, we provide a more general strategy for mimicking noncanonical transformations that should broaden their use in organic synthesis.

\section{INTRODUCTION}

Many of the unique shapes and functional properties of natural products emerge in the late stages of biosynthesis following $\mathrm{C}-\mathrm{H}$ oxidation. ${ }^{1}$ Cytochrome P450s and non-heme iron oxygenases that carry out these transformations have been the focus of many studies aiming to emulate their mechanisms of $\mathrm{C}-\mathrm{H}$ oxidation, as well as their timing in synthetic strategy. ${ }^{2-3}$ While the majority of examples studied thus far involve $\mathrm{C}-\mathrm{H}$ hydroxylation by an established mechanism of rebound, ${ }^{3}$ there is growing awareness of noncanonical oxygenases that divert this pathway to create more pronounced structural changes (Scheme 1A). ${ }^{4-5}$ Examples include the ansa-bridge of streptorubin $B,{ }^{6}$ the aryltetralin of (-)-deoxypodophyllotoxin ${ }^{7}$ and the fused macrocycles of vancomycin ${ }^{8}$ (Scheme 1B), in which oxidative cyclizations rigidify relatively flexible substrates to create the unique three dimensional architectures of the natural products. Because they create the defining structural features that lead to complexity, ${ }^{9}$ these transformations can be strategic targets for retrosynthetic simplification. However, unlike canonical hydroxylation, for which there is a deep mechanistic understanding and many synthetic alternatives, ${ }^{1,3}$ the mechanisms of noncanonical oxidations remain poorly understoo ${ }^{10}$ and the transformations themselves are difficult to replicate in the absence of the associated enzymes. ${ }^{11}$ This creates a significant hurdle for their incorporation into a synthetic plan, particularly when the associated enzyme is either unknown or difficult to express in a synthetically useful form. ${ }^{11}$ In these cases, a synthetic equivalent that could reconstitute the structural changes under practical conditions would be a welcome alternative, which might also provide insights into the mechanisms of these poorly understood transformations. 


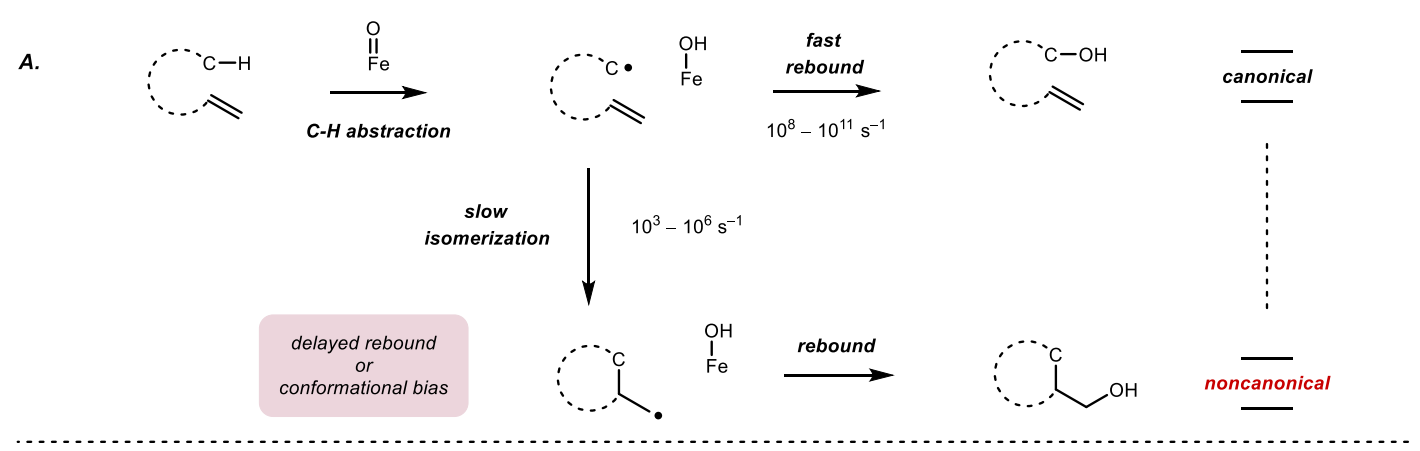

B.
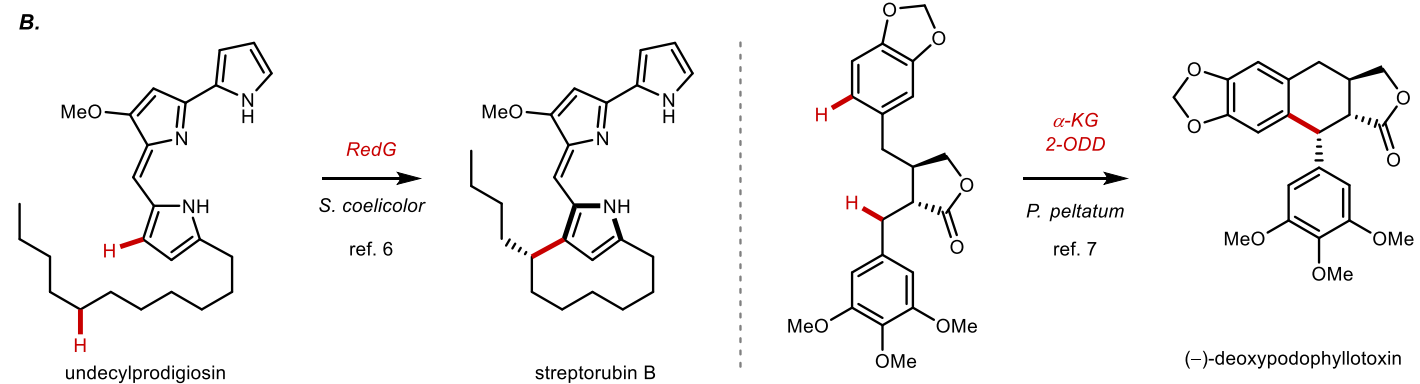

(-)-deoxypodophyllotoxin
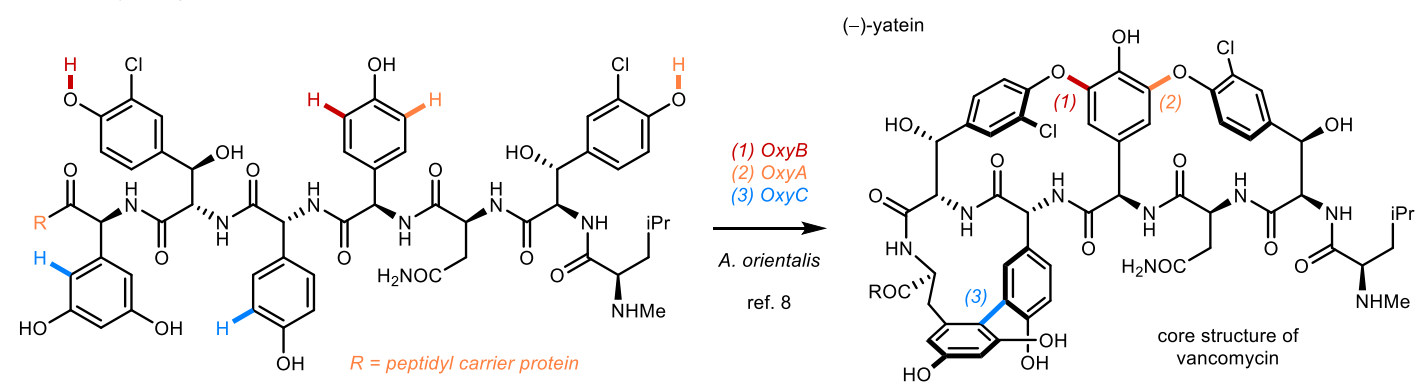

Scheme 1. (A) Canonical and non-canonical oxidation mediated by iron-containing oxygenases. (B) Examples of noncanonical oxidations in the biosynthesis of streptorubin $B,(-)$-deoxypodophyllotoxin and vancomycin.

Our interest in developing such a tool stems from a striking example of noncanonical biosynthesis that occurs in medicinal plants of Kadsura (Scheme 2A). In a remarkable display of divergent biosynthesis, ${ }^{12}$ the dibenzocyclooctadienes (DBCODs) ${ }^{13}$ schizanrin $F(\mathbf{1})$ and kadsurarin (2) are oxidized on their phenyl-methyl ethers to create the defining 5- and 11-membered rings of heteroclitin J (3) ${ }^{14}$ and kadsuphilin $\mathrm{N}(4),{ }^{15}$ respectively. The intricate framework of the taiwankadsurins $(\mathbf{5 a} / \mathbf{5 b})^{16}$ is then further developed by oxidative cleavage of the dienone in 3, to complete the deconstructive functionalization that diversifies the biphenyl into the bridging pyran. ${ }^{17-18}$ While the oxidation of phenyl-methyl ethers to phenoxy methyl radicals is a relatively common metabolic step, it typically affords phenols by a canonical mechanism of hydroxylation and fragmentation. ${ }^{5}$ Therefore, the cyclizations leading to $\mathbf{3}$ and $\mathbf{4}$ raise questions about how the associated oxygenases delay or divert rebound to create these more pronounced structural changes. Rebound $\left(10^{8}-10^{11} \mathrm{~s}^{-1}\right)^{3}$ is typically faster than radical cyclization $\left(10^{3}-10^{6} \mathrm{~s}^{-1}\right)^{19}$ by several orders of magnitude, suggesting that these enzymes either segregate the phenoxy-methyl radical from the Fe-(III)-OH to delay rebound, or accelerate cyclization by creating a conformational bias. This mechanistic difference has important implications for biomimicry, since recreating the secondary interactions of a protein's active site in order to change a molecule's conformational preferences remains quite challenging. ${ }^{20}$ Instead, a chemical synthesis could more readily attempt to mimic these noncanonical steps by relying on the innate reactivity of the proposed biosynthetic radicals, under the assumption that they undergo these relatively challenging cyclizations in the absence of an external conformational bias. 

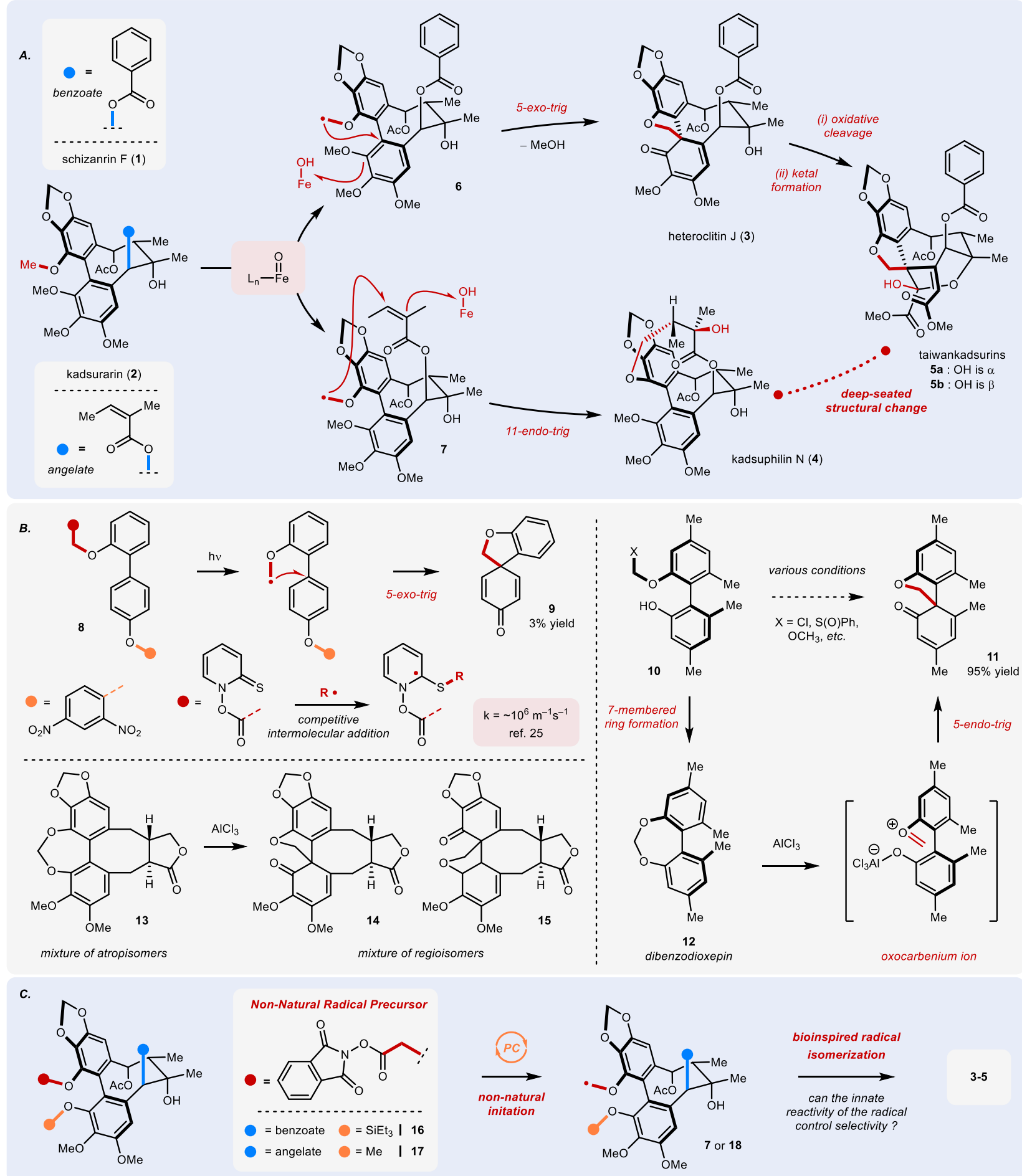

Scheme 2. (A) Biosynthetic hypothesis for 1-3 via noncanonical oxidation. (B) Previous studies of the 5-membered spirocyclization. (C) This work: mimicking biosynthetic noncanonical oxidations using photoredox catalysis.

While there has not been a total synthesis of an oxidized DBCOD family member, there have been three studies examining the 5-membered spirocyclization (Scheme 2B). A radical mechanism consists of a stereoelectronically allowed 5-exo-trig cyclization, but requires a nucleophilic carbon centered radical to add to an electron rich aromatic ring. This is an uncommon, polarity mismatched radical addition ${ }^{19,21}$ that was investigated by Whiting and coworkers in 1998. ${ }^{22}$ Using Barton's conditions for the generation of the phenoxy-methyl radical and a 2,4-dinitro 
arene as a radical trap, they reported only a 3\% yield for the spirocyclization of $\mathbf{8}$ to spirodienone $\mathbf{9}$. Coleman and co-workers subsequently investigated a polarity aligned, cationic alternative, recognizing that the biosynthetic cyclization might involve oxidation of the phenoxy-methyl radical to the oxocarbenium ion prior to cyclization. Similar mechanistic proposals have been made for other noncanonical oxygenases, reflecting persistent questions about the Fe-(III)-OH that is formed following $\mathrm{C}-\mathrm{H}$ abstraction, and whether it can oxidize radicals to cations as a means of avoiding rebound. ${ }^{5-6,10}$ There is also the possibility that these enzymes do not avoid rebound, and that cationic mechanisms of cyclization initiate from alcohols by more classical Lewis acid activation. Coleman's attempts to cyclize biphenyl ethers $\mathbf{1 0}$ representative of this pathway uncovered a competitive 7-membered ring formation that led selectively to dibenzodioxepin 12 . The stereoelectronically disfavored 5-endo-trig cyclization onto the oxocarbenium ion could only be effected in a second step by treating $\mathbf{1 2}$ with a strong Lewis acid. ${ }^{23}$ While successful in a symmetrical model system, subsequent efforts by Baran and co-workers to use these conditions on a nonsymmetrical substrate $\mathbf{1 3}$, more appropriate for a synthesis of $\mathbf{3}$, led to a complex mixture of products that reflected poor regioselectivity in the ionization of the dibenzodioxepin. ${ }^{24}$

In light of these studies, we sought to mimic the noncanonical steps leading to 3-5 by generating the presumed biosynthetic radicals under conditions that could better tolerate relatively slow carbocyclizations. We noted that Barton's conditions are susceptible to a competitive radical addition to the thiocarbonyl of the starting material, which occurs with a second order rate constant of $\sim 10^{6} \mathrm{~m}^{-1} \mathrm{~s}^{-1}$ (Scheme 2B). ${ }^{25}$ Therefore, we considered more recently developed photoredox mediated conditions, ${ }^{26}$ in which carbon centered radicals are formed by one electron reduction of an ( $\mathrm{N}$-acyloxy)-phthalimide (NHPI) redox-active ester (Scheme $2 \mathrm{C}$ ). ${ }^{27}$ By generating the phenoxy methyl radical catalytically under redox neutral conditions, we hoped to approximate the biosynthetic environment of the oxygenase's active site, under the assumption that it prolongs radical lifetime without necessarily influencing its innate reactivity. This led us to identify radical progenitors $\mathbf{1 6}$ and $\mathbf{1 7}$ as strategic molecules from which to explore this hypothesis.

Before being able to evaluate these radical cyclizations, we needed to address a longstanding challenge of atroposelective biaryl bond formation, which has been a defining feature of the DBCOD literature (Scheme 3). ${ }^{13,28}$ The particular challenge, as it relates to $\mathbf{1 6}$ and $\mathbf{1 7}$, is the relative $(P)$-configuration of the biaryl axis and the configurations of the benzylic esters at $\mathrm{C} 6$ and C9. Both reside in pseudo axial positions of the twist-boat-chair conformation, and, as configured, create a conformational bias that would favor the opposite atropisomer $(M, 6 S, 9 R)-16 \mathrm{~b}$ or $17 \mathrm{~b}$ in the cyclization of a linear precursor $19 .{ }^{29}$ This challenge in medium-sized ring formation has not been solved by previous DBCOD syntheses, and helps to explain why there is only a single previous synthesis of a "fully-substituted" DBCOD, containing the complete stereotetrad in the 8-membered ring. ${ }^{30}$ Therefore, to address this challenge, we chose to invert the stereochemistry at $\mathrm{C} 6$ and tether the benzylic alcohol at $\mathrm{C} 9$, in anticipation that these changes would allow a stereoselective intramolecular cyclization (Scheme 3). 


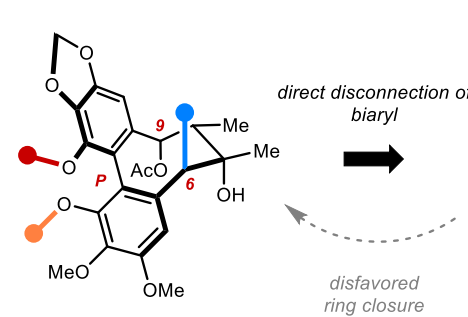

$(P, 6 S, 9 R)-16$ a or 17a

(1) epimerize $C 6$

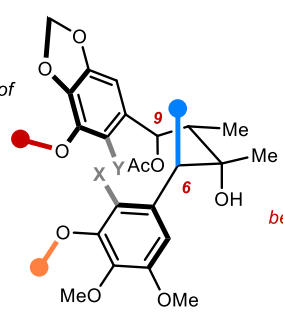

19-a required but disfavored conformation
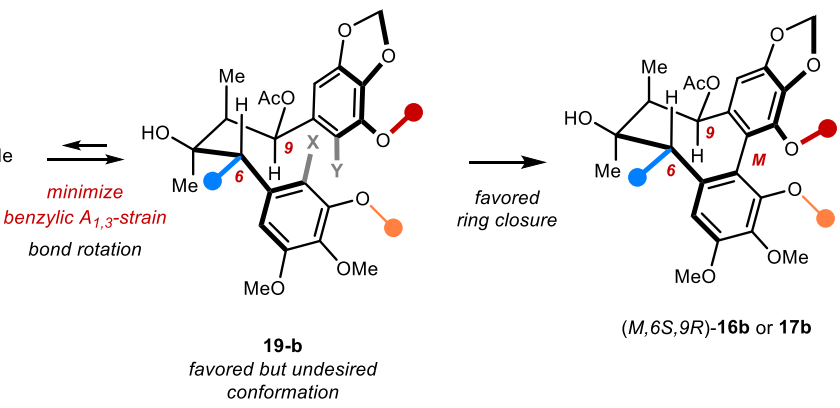

$(M, 6 S, 9 R)-16 b$ or $17 \mathbf{b}$ conformation

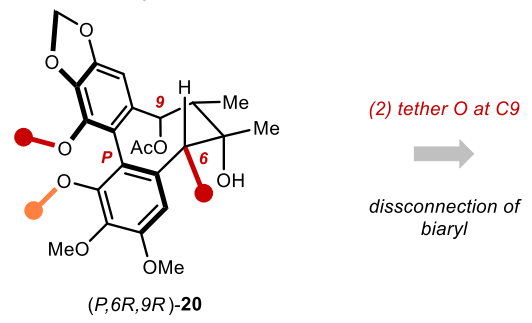

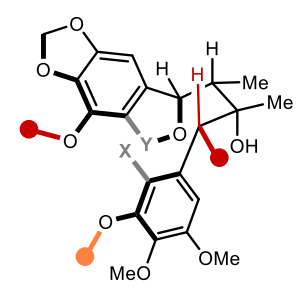

$\bar{\equiv}$

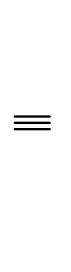

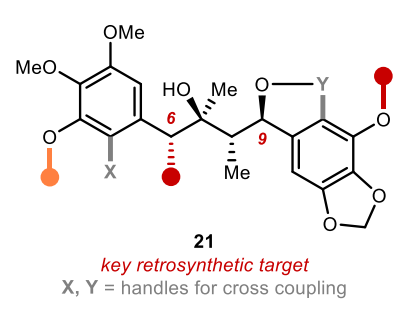

Scheme 3. Retrosynthetic analysis for the atroposelective biaryl bond formation.

Our syntheses of $\mathbf{1 6}$ and $\mathbf{1 7}$ began by a 4-step preparation of $(E)-\mathrm{Cy}_{2} \mathrm{~B}$-enolate $\mathbf{2 5}$, in which the benzylic stereocenter at C6 (DBCOD numbering) was set in 93\% ee using North's catalytic asymmetric hydrocyanation (Scheme 4A). ${ }^{31}$ This stereocenter was then used to control the ensuing anti-aldol (TS-I) with good 1, 3-syn-induction (20:1 dr) to provide 27 in $66 \%$ yield and high enantiopurity ( $>99 \%$ ee) following recrystallization on $13 \mathrm{~g}$ scale. Tertiary alcohol $\mathbf{2 8}$ was then prepared under Bartoli's conditions in a 70\% yield with 3:1 diastereoselectivity on $14 \mathrm{~g}$ scale by pseudo-axial addition of a methyl nucleophile to the 6-membered titanium chelate (TS-II). ${ }^{32}$ In preparation for the ensuing biaryl bond formation, we installed a cyclic borinic ester to tether the C9-alcohol over a two-step sequence, and then selectively deprotected the phenol to direct halogenation at C16. ${ }^{33}$ The ensuing intramolecular Suzuki coupling was

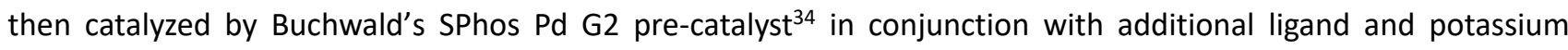
phosphate $\left(\mathrm{K}_{3} \mathrm{PO}_{4}\right)$ to afford 30 in $87 \%$ yield on $6 \mathrm{~g}$ scale. Given the well-documented challenges of forming tetrasubstituted biaryl bonds stereoselectively, ${ }^{28}$ the efficiency of these conditions is noteworthy. We believe that the $(P)$-configuration of the chiral axis results from a conformational bias to minimize $\mathrm{A}_{1,3}$-strain at $\mathrm{C} 6$, and the confinement of the $\mathrm{C9}-\mathrm{O}$ bond within the borinic ester (Scheme 4B). This conformation should be maintained in a stereodetermining transmetalation (TS-III), ${ }^{35}$ which ensures a syn co-planar orientation of the C9-O bond and the forming biaryl axis, as well as a conformation that eclipses the aryl-Pd-(II) and the benzylic hydrogen at $\mathrm{C} 6$ to minimize steric interactions with the silyl ether. From 30, we then used a series of functional group manipulations to install the acetate at $\mathbf{C 9}$, invert the stereochemistry at $\mathbf{C 6}$, and retain differentiation of the phenyl ethers, providing a point of divergence in the form of $\mathbf{3 1}$. Carboxylic acids $\mathbf{3 3}$ and $\mathbf{3 6}$ were then prepared over 5-step sequences that incorporated benzoate or angelate esters at $\mathrm{C} 6$, and either $\mathrm{O}$-silyl or $\mathrm{O}$-Me substitution at $\mathrm{C} 1$. These stable radical progenitors could be prepared on gram scale over a longest linear sequence of 20 steps. 


$$
\text { A. }
$$
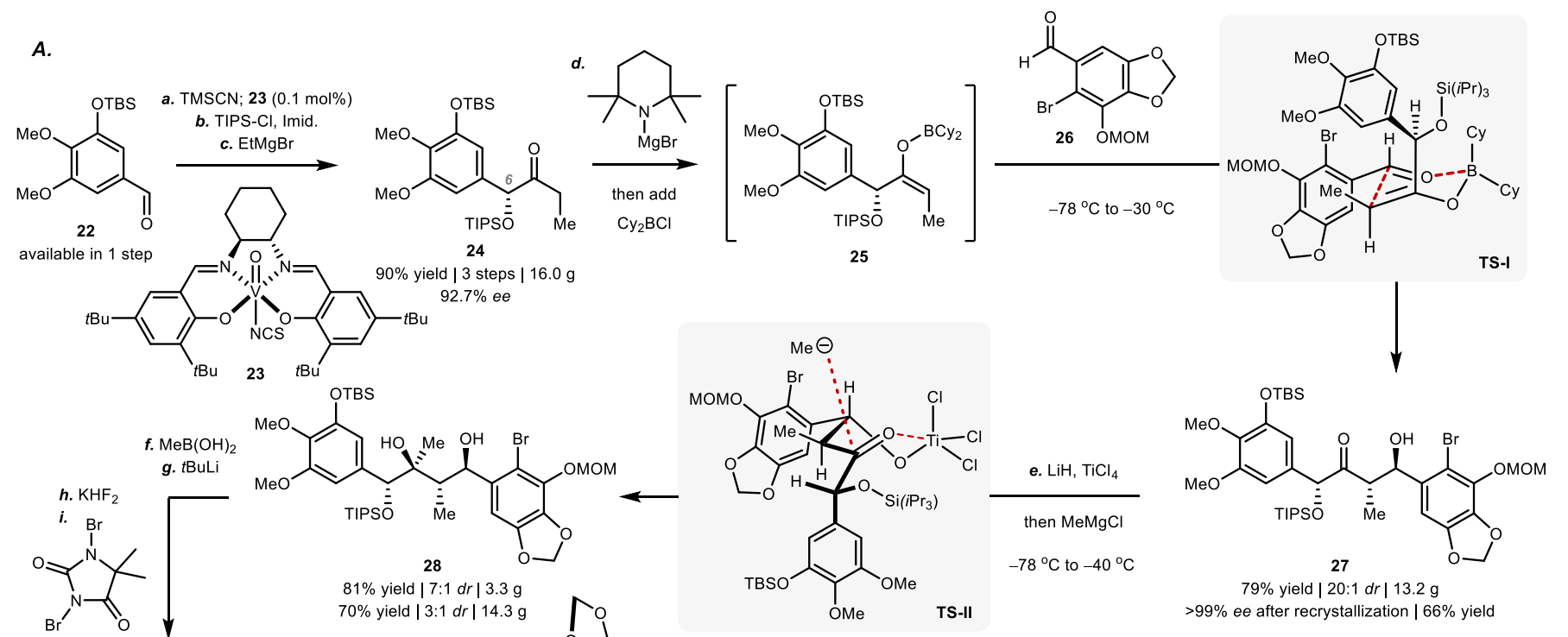

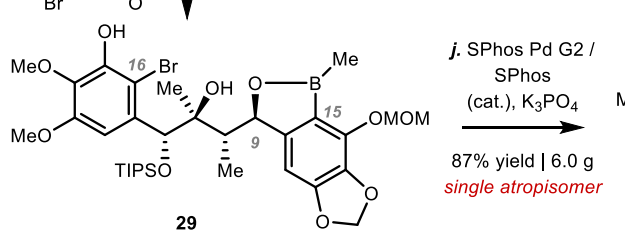

$76 \%$ yield $\mid 4$ steps $\mid 10.9 \mathrm{~g}$

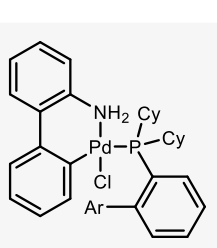

$\mathrm{Ar}=2,6-\mathrm{MeO}-\mathrm{C}_{6} \mathrm{H}_{3}$ SPhos $\mathrm{Pd} \mathrm{G} 2$
S

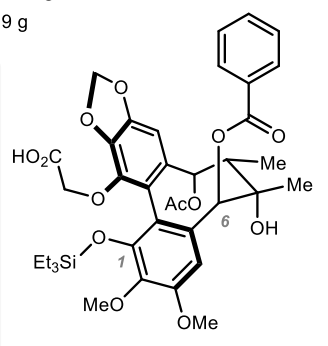

33

$51 \%$ yield $\mid 5$ steps $\mid 1.1 \mathrm{~g}$

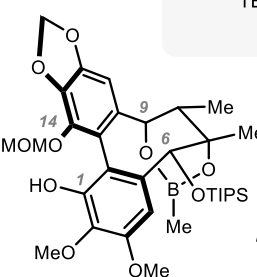

30

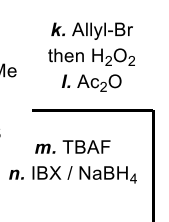

$\downarrow$

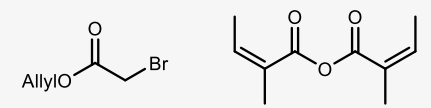

34

32

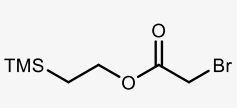

35

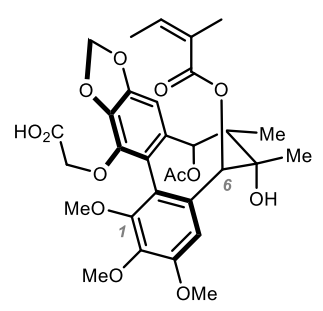

36

$55 \%$ yield | 5 steps $\mid 90 \mathrm{mg}$

s.

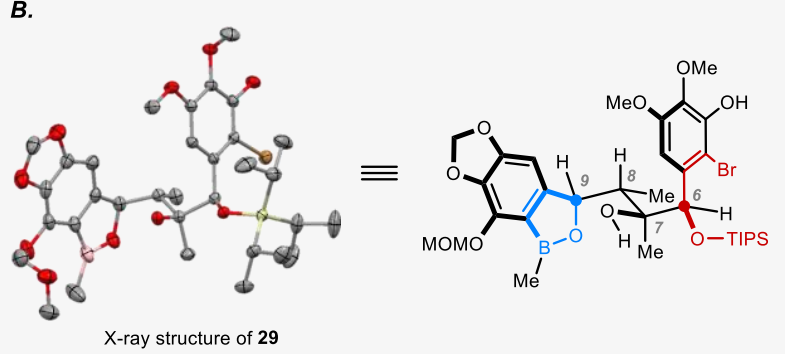

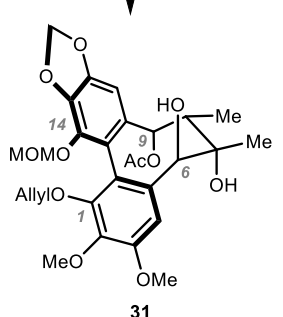

$56 \%$ yield $\mid 4$ steps $\mid 2.8 \mathrm{~g}$

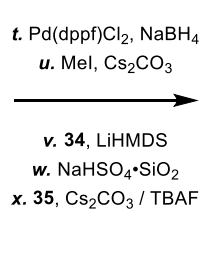

v. 34, $\mathrm{LiHMDS}$
w. $\mathrm{NaHSO}_{4} \cdot \mathrm{SiO}_{2}$ x. 35, $\mathrm{Cs}_{2} \mathrm{CO}_{3} /$ TBAF

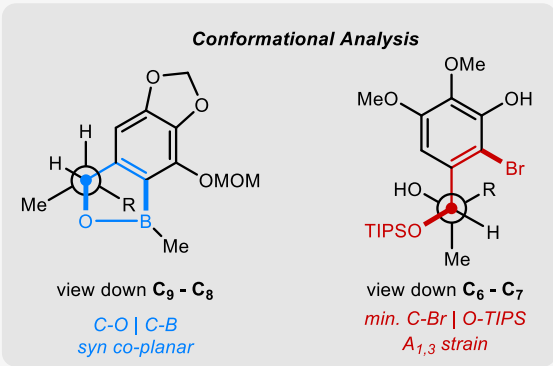

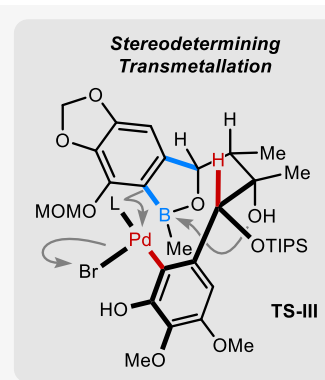

Scheme 4. (A) Forward synthesis of radical precursors. (B) Conformational analysis of the atroposelective biaryl coupling. Reagents and conditions: a) 23 (0.1 mol\%), TMSCN (1.2 equiv), $\mathrm{CH}_{2} \mathrm{Cl}_{2}, \mathrm{O}_{2},-20^{\circ} \mathrm{C}, 24$ hours, then $\mathrm{H}_{3} \mathrm{PO}_{4} / \mathrm{FeSO}_{4}, \mathrm{MeOH}^{\mathrm{rt}}$, $15 \mathrm{~min}, 99 \%$ yield, 93\% ee; b) TIPSCl (1.2 equiv), imidazole (1.5 equiv), DMF, rt, 18 hours, 96\%; c) $\mathrm{EtMgBr}(1.5 \mathrm{equiv})$, $\mathrm{CH}_{2} \mathrm{Cl}_{2}, 0^{\circ} \mathrm{C}$-rt, 4 hours, then $\mathrm{HCl}, 0^{\circ} \mathrm{C}$-rt, 1 hour, 94\%; d) 2,2,6,6-tetramethylpiperidine (1.68 equiv), $\mathrm{EtMgBr}$ (1.5 equiv), reflux, 12 hours, then 24 (1.0 equiv), $-78{ }^{\circ} \mathrm{C}, 1$ hour, then $\mathrm{Cy}_{2} \mathrm{BCl}$ (1.2 equiv), $-78^{\circ} \mathrm{C}-\mathrm{rt}$, 1 hour, then 26 (1.0 equiv), -78 ${ }^{\circ} \mathrm{C}, 3$ hours then $-30{ }^{\circ} \mathrm{C}, 2$ hours, $79 \%$ of a $20: 1 d r$ mixture, then 2 recrystallizations from hexanes, 66\%, >99\% ee; e) LiH (1.5 equiv), $\mathrm{TiCl}_{4}\left(2.0\right.$ equiv), $\mathrm{THF},-30{ }^{\circ} \mathrm{C}, 30 \mathrm{~min}$, then $\mathrm{MeMgCl}\left(4.0\right.$ equiv), $-78{ }^{\circ} \mathrm{C}, 1$ hour, then $-40{ }^{\circ} \mathrm{C}, 2$ hours, $70 \%$ yield, 3:1 dr; f) $\mathrm{MeB}(\mathrm{OH})_{2}$ (1.2 equiv), $4 \AA$ Mol. Sieve, $\mathrm{CH}_{2} \mathrm{Cl}_{2}, \mathrm{rt}, 12$ hours, 97\%; g) ${ }^{t} \mathrm{BuLi}\left(2.0\right.$ equiv), $\mathrm{THF},-78^{\circ} \mathrm{C}, 1$ hour, then rt, 3 hours; h) $\mathrm{KHF}_{2}$ (2.0 equiv), MeOH/THF, rt, 1 hour, $81 \%$ over 2 steps; i) DBDMH (0.5 equiv), $\mathrm{CHCl}_{3}, 0^{\circ} \mathrm{C}, 30 \mathrm{~min}$, 97\%; j) SPhos Pd G2 (20 mol\%), SPhos (20 mol\%), $\mathrm{K}_{3} \mathrm{PO}_{4}$ (5 equiv), PhMe, $60{ }^{\circ} \mathrm{C}, 16$ hours, 87\%; k) allyl bromide (1.5 equiv), $\mathrm{Cs}_{2} \mathrm{CO}_{3}$ (2.0 equiv), DMSO, rt, 12 hours, then $\mathrm{H}_{2} \mathrm{O}_{2}$ (1.5 equiv), $\mathrm{NaOH} / \mathrm{MeOH}, 0^{\circ} \mathrm{C}, 2$ hours, 95\%; I) DMAP (20 mol\%), Ac $\mathrm{O}$ 
(10 equiv), pyridine (10 equiv), $\mathrm{CH}_{2} \mathrm{Cl}_{2}, \mathrm{rt}, 18$ hours; $\mathrm{m}$ ) TBAF· $3 \mathrm{H}_{2} \mathrm{O}$ (3 equiv), $\mathrm{HOAc}$ (1.2 equiv), THF, rt, 24 hours, $73 \%$ over 2 steps; $n$ ) IBX (1.2 equiv), DMSO, rt, 3 hours, then $\mathrm{NaBH}_{4}$ (3.0 equiv), $\mathrm{MeOH}, 0{ }^{\circ} \mathrm{C}, 15 \mathrm{~min}, 81 \%$; o) DMAP (1.0 equiv), BzCl (6.0 equiv), DIPEA (2.0 equiv), pyridine, $60{ }^{\circ} \mathrm{C}, 18$ hours, 89\%; p) $\mathrm{NaHSO}_{4} \cdot \mathrm{SiO}_{2}, \mathrm{CH}_{2} \mathrm{Cl}_{2}, \mathrm{rt}, 6$ hours; q) 32 (1.5 equiv), $\mathrm{Cs}_{2} \mathrm{CO}_{3}$ (2.0 equiv), DMSO, rt, 2 hours, 71\% over 2 steps; r) Pd(dppf)Cl 2 (10 mol\%), $\mathrm{H}_{2} \mathrm{O}$ (50 mol\%), $\mathrm{NaBH}_{4}$ (4.0 equiv), THF, rt, 4 hours; s) TESCl (10 equiv), imidazole (12 equiv), DMF/ $\mathrm{CH}_{2} \mathrm{Cl}_{2}, \mathrm{rt}, 12$ hours, then aq. citric acid, $\mathrm{rt}, 10 \mathrm{~min}, 80 \%$ over 2 steps; t) $\mathrm{Pd}(\mathrm{dppf}) \mathrm{Cl}_{2}$ (2 mol\%), $\mathrm{H}_{2} \mathrm{O}$ (50 mol\%), $\mathrm{NaBH}_{4}$ (3.0 equiv), THF, rt, 1 hour; u) Mel (2.0 equiv), $\mathrm{Cs}_{2} \mathrm{CO}_{3}$ (2.0 equiv), DMSO, rt, 2 hours, 96\% over 2 steps; v) 34 (1.5 equiv), LiHMDS (1.2 equiv), THF, $0{ }^{\circ} \mathrm{C}, 1$ hour, 78\%; w) $\mathrm{NaHSO}_{4} \cdot \mathrm{SiO}_{2}, \mathrm{CH}_{2} \mathrm{Cl}_{2}, \mathrm{rt}, 3$ hours; x) 35 (1.5 equiv), $\mathrm{Cs}_{2} \mathrm{CO}_{3}$ (2.0 equiv), DMSO, rt, 2 hours, then TBAF·3 $\mathrm{H}_{2} \mathrm{O}$ (3.0 equiv), THF, rt, 2 hours, $73 \%$ over 2 steps.

To investigate the key radical cyclization that would afford spirodienone $\mathbf{3}$ (Scheme 5A), we esterified acid 33 with NHPI using diisopropylcarbodiimide (DIC) in dichloromethane $\left(\mathrm{CH}_{2} \mathrm{Cl}_{2}\right)$ before adding 1 mol\% of tris(2,2'bipyridine)ruthenium(II) hexafluorophosphate $\left[\mathrm{Ru}(\mathrm{bpy})_{3}\left(\mathrm{PF}_{6}\right)_{2}\right]$ and 2 equivalents of triethylamine $\left(\mathrm{Et}_{3} \mathrm{~N}\right)$ as a solution in dimethylformamide (DMF). Subsequent irradiation with blue LEDs resulted in a successful spirocyclization that returned heteroclitin $\mathrm{J}(3)^{14}$ in an isolated yield of $88 \%$ on $250 \mathrm{mg}$ scale. Based on previous studies of similar photocatalytic reactions, ${ }^{27}$ we believe that $\mathrm{Et}_{3} \mathrm{~N}$ reduces photo-excited $\mathrm{Ru}$-(II) to Ru-(I), which in turn reduces the $\mathrm{N}$-phthalimidoyl ester of $\mathbf{1 6}$ to trigger fragmentation. This would release phthalimide anion, carbon dioxide $\left(\mathrm{CO}_{2}\right)$ and the phenoxy-methyl radical 18, before installation of the quaternary stereocenter by 5-exo-trig spirocyclization. Oxidation of the subsequent radical $\mathbf{3 7}$ by the triethylaminyl radical cation would then close the electron transfer loop, before transfer of the triethylsilyl group to the phthalimide anion. We do not observe dibenzodioxepin or regioisomeric products that we would expect from cationic intermediates, providing support for a radical mechanism of spirocyclization.

Since the addition of radicals to electron rich aromatic rings is uncommon, we were intrigued by the high efficiency for spirocyclization, and whether a conformational bias imposed by the DBCOD core could be overcome in order to form the 11-membered ring required for 4 . We explored this question by subjecting angelate ester 17 to similar photoredox conditions, and gratifyingly obtained a 1:1 mixture of alkane $\mathbf{3 8}$ and alkene $\mathbf{3 9}$ in a combined yield of $57 \%$ at complete consumption of the starting material (Scheme 5B). We did not detect any amount of spirodienone by-products, suggesting high selectivity for the electronically favored 11-membered ring formation, and we attributed the $\sim 40 \%$ loss of mass balance to decomposition of $3^{\circ}$ radical $\mathbf{4 0}$ following macrocyclization. The formation of $\mathbf{3 8}$ and $\mathbf{3 9}$ is consistent with the disproportionation of $\mathbf{4 0}$, which creates $\mathbf{3 8}$ as a $5: 1$ mixture of inseparable diastereomers at $\mathrm{C2}^{\prime}$. The $(S)$-configuration at C3' is installed selectively for both $\mathbf{3 8}$ and $\mathbf{3 9}$, which would be expected for a transannular cyclization involving the thermodynamically preferred s-cis conformation of the angelate ester (Scheme 5B, inset). While the biosynthesis of 4 terminates macrocyclization by rebound exo to the macrocycle, we were unable to conduct a similar termination by $\mathrm{C}-\mathrm{X}$ bond formation ( $\mathrm{X}=\mathrm{O}$ or Se), which instead returned products of premature termination without macrocyclization. Therefore, we developed a macrocyclization that could afford olefin $\mathbf{3 9}$ more cleanly, under the assumption that we could install the $3^{\circ}$ hydroxyl group of the natural product in a subsequent step. To this end, we explored Fu's recently developed metallaphotoredox conditions, ${ }^{26,36-37}$ with the goal of terminating macrocyclization via a more programmable ${ }^{38}$ process. Once again, irradiation of 17, but this time in the presence of bis(triphenylphosphine)palladium(II) dichloride $\left[\mathrm{Pd}\left(\mathrm{PPh}_{3}\right)_{2} \mathrm{Cl}{ }_{2}\right](10$ mol\%), XantPhos (12 mol\%), water $\left(\mathrm{H}_{2} \mathrm{O}\right)$ and potassium carbonate $\left(\mathrm{K}_{2} \mathrm{CO}_{3}\right)$ in dimethylacetamide (DMA) afforded 39 in an improved yield of 53\% (72\% based on recovered starting material), and only trace amounts of $\mathbf{3 8}$. We attribute this change in selectivity to the coordination of $\mathrm{Pd}$, which provides a better-behaved closure to the catalytic cycle by $\beta$-hydride elimination (Scheme $5 B$, inset). 


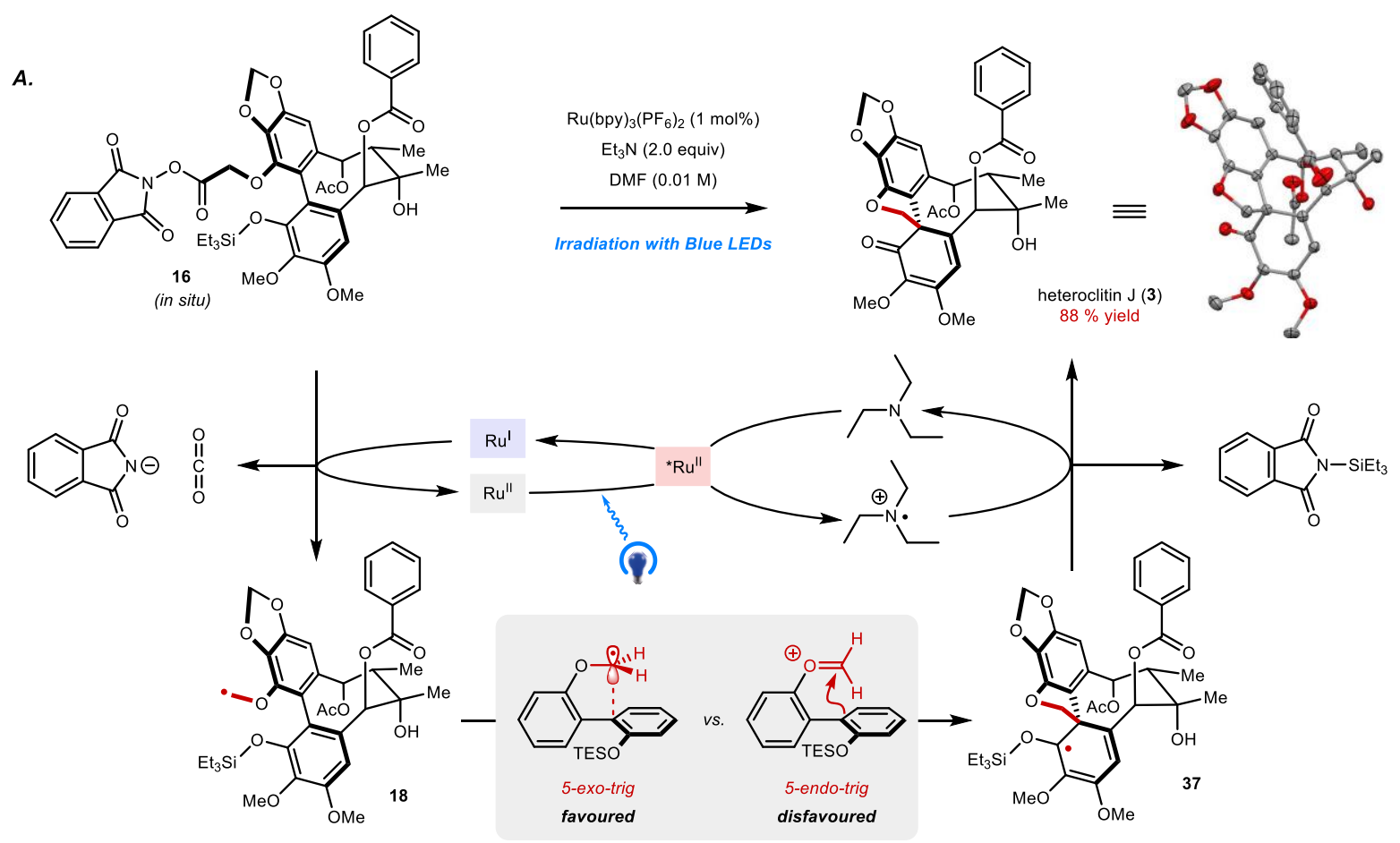

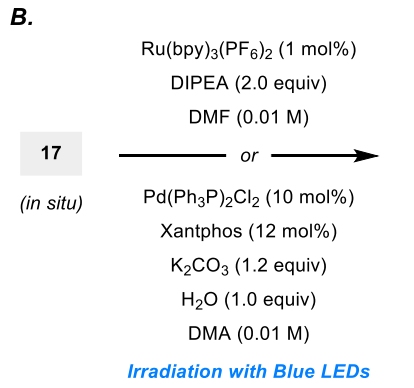

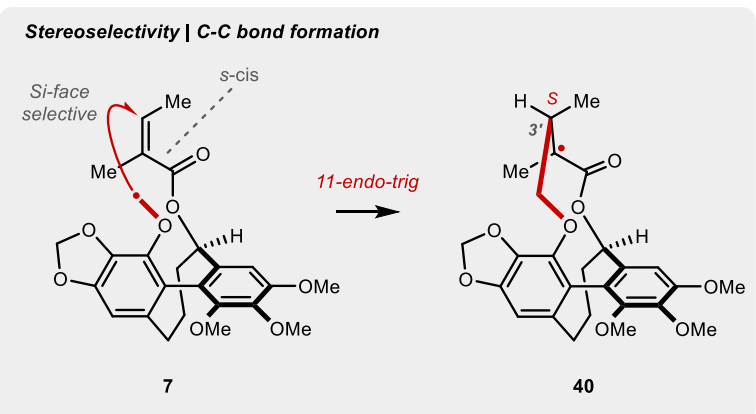

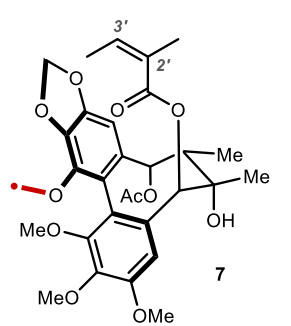

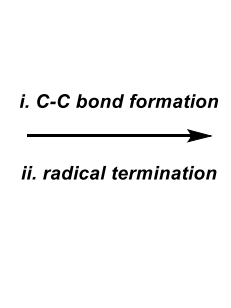

$\mathrm{Ru}$

$\mathrm{Pd}$

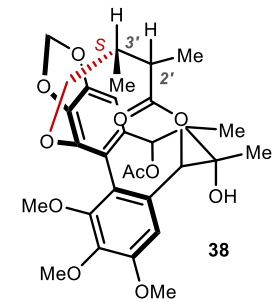

$30 \%$ yield | $d r 5: 1$ at $\mathrm{C} 2^{\prime a}$

$<5 \%$ yield

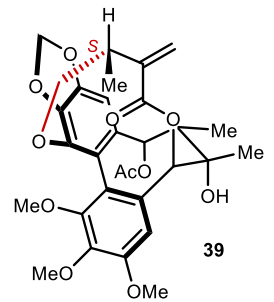

$27 \%$ yield | single diastereomer ${ }^{a}$ $53 \%$ yield $\mid$ single diastereomer ${ }^{b}$

Radical Termination
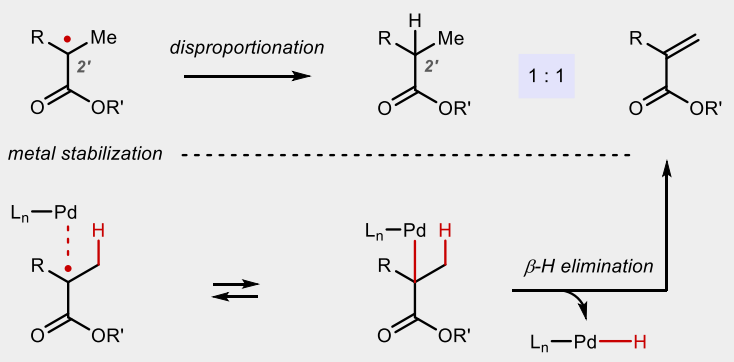

Scheme 5. (A) Synthesis of heteroclitin J (3), and proposed mechanism of photoredox catalyzed radical cyclization. (B) Stereoselective 11-endo-trig radical cyclization controlled by metallaphotoredox catalysis. ${ }^{(a)}$ Yield and diastereomeric ratio (dr) determined by ${ }^{1} \mathrm{H}-\mathrm{NMR}$ analysis of the crude reaction mixture using ethylene carbonate as an internal standard. (b) Isolated yield.

To complete the synthesis of taiwankadsurins $A$ and $B(5 a / b)$, we cleaved the most electron rich double bond of the spirodienone by ozonoloysis using a reductive work-up (Scheme 6). The resulting keto-ester $\mathbf{4 1}$ was then susceptible to hemi-ketalization with the $3^{\circ}$ alcohol, creating the bridging pyran of the final polycyclic framework. Taiwankadsurins $\mathbf{5 a / b}$ were formed as a mixture of separable diastereomers in a combined yield of $70 \%$ and a 9:1 ratio that is similar to the ratio isolated by Shen and co-workers from Kadsura philippinensis. ${ }^{16}$ To complete the 
synthesis of kadsuphilin N (4) from 39, we employed a Mukaiyama hydration, ${ }^{39}$ which installed the $3^{\circ}$ alcohol at $\mathrm{C2}^{\prime}$ as a 5:1 mixture of separable diastereomers in favor of the natural $(R)$-configuration. The stereoselectivity of hydration was consistent with the addition of a $\mathrm{Mn}-\mathrm{H}$ to the pro- $(R)$-face of the olefin, followed by stereoretentive oxygenation. ${ }^{40}$ We wish to highlight the combination of metallaphotoredox and Mukaiyama hydration as a redox neutral and stereoselective equivalent to the biosynthetic cyclization that could find more general use for the carbohydroxylation of olefins. Overall, our syntheses of $5 \mathrm{a} / \mathbf{b}$ and $\mathbf{4}$ require longest linear sequences of 22 steps, and proceed in $5.0 \%$ and $3.4 \%$ overall yields, respectively. The routes have sustained late-stage optimization studies and provided tens of milligrams of the natural products in high purity, completing the first total syntheses of such highly oxidized DBCOD family members.

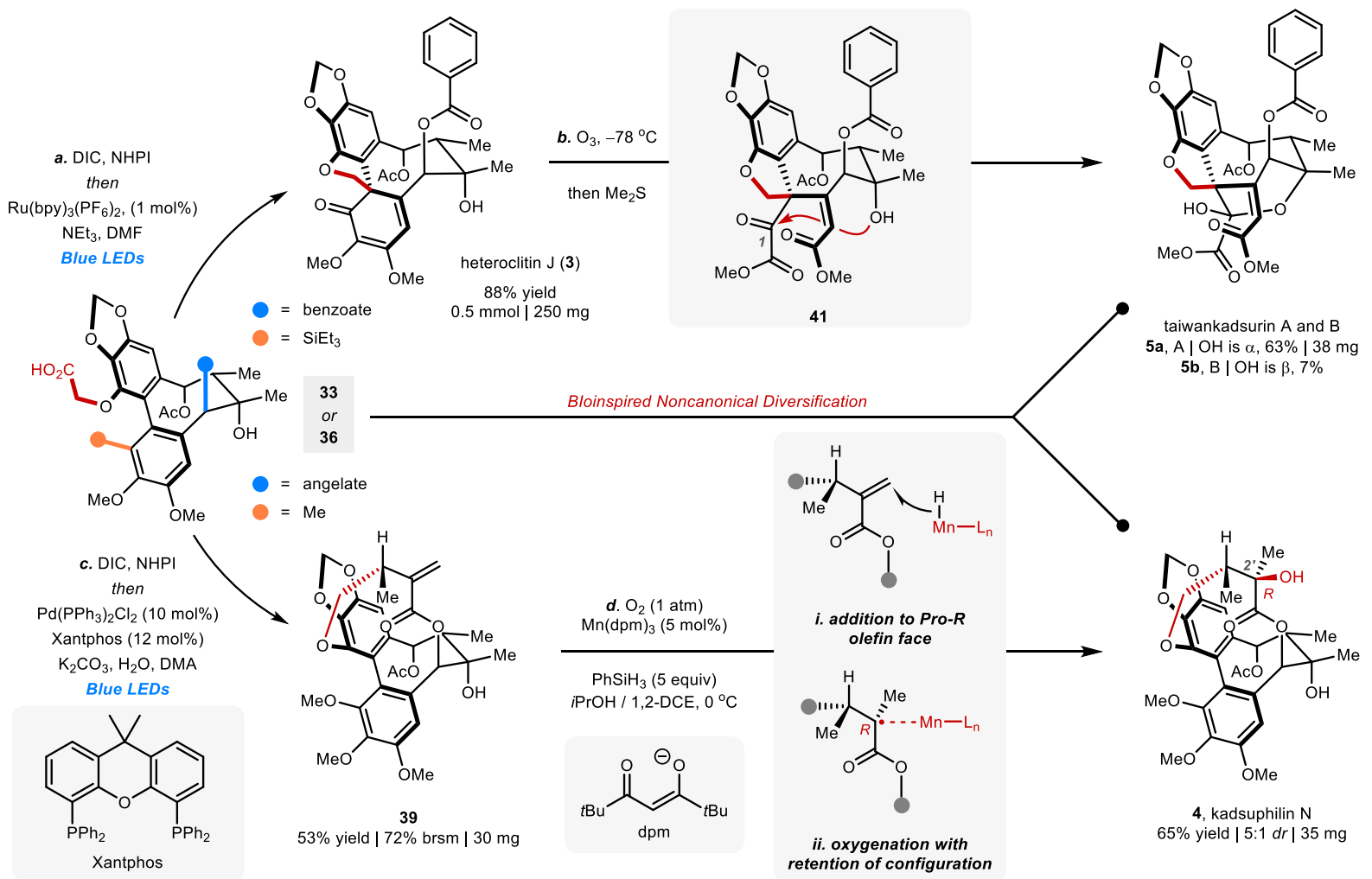

Scheme 6. Synthesis of taiwankadsurin A (5a) and B (5b), and kadsuphilin N (4). Reagents and conditions: a) NHPI (1.1 equiv), DIC (1.2 equiv), $\mathrm{CH}_{2} \mathrm{Cl}_{2}, \mathrm{rt}, 12$ hours, then $\mathrm{Ru}(\mathrm{bpy})_{3}\left(\mathrm{PF}_{6}\right)_{2}$ (1 mol\%), $\mathrm{NEt}_{3}$ (2.0 equiv), DMF, rt, 3 hours, Blue LEDs, $88 \%$; b) $\mathrm{O}_{3} / \mathrm{O}_{2}, \mathrm{CH}_{2} \mathrm{Cl}_{2},-78{ }^{\circ} \mathrm{C}$, 5 seconds, then $\mathrm{Me}_{2} \mathrm{~S},-78{ }^{\circ} \mathrm{C}-\mathrm{rt}, 1$ hour, $63 \% 5 \mathbf{a}+7 \% \mathbf{5 b}$; c) NHPI (1.1 equiv), DIC (1.2 equiv), $\mathrm{CH}_{2} \mathrm{Cl}_{2}, \mathrm{rt}, 12$ hours, then $\mathrm{Pd}\left(\mathrm{PPh}_{3}\right)_{2} \mathrm{Cl}_{2}(10 \mathrm{~mol} \%)$, Xantphos (12 mol\%), $\mathrm{K}_{2} \mathrm{CO}_{3}$ (1.2 equiv), $\mathrm{H}_{2} \mathrm{O}$ (1.0 equiv), DMA, $\mathrm{rt}, 8$ hours, $53 \%$ 39, 72\% brsm; d) $\mathrm{O}_{2}(1 \mathrm{~atm}), \mathrm{Mn}(\mathrm{dpm})_{3}(5 \mathrm{~mol} \%), \mathrm{PhSiH}_{3}\left(5\right.$ equiv), ${ }^{i} \mathrm{PrOH} / 1,2-\mathrm{DCE}(1: 1), 0^{\circ} \mathrm{C}, 4 \mathrm{~h}$, then $\mathrm{PPh}_{3}$ (1.5 equiv), rt, $30 \mathrm{~min}, 65 \% 4+13 \% \mathrm{C2}^{\prime}$ epimer, 5:1 dr.

\section{CONCLUSION}

The successful mimicry of noncanonical cyclizations using photoredox catalysis suggests that related biosynthetic transformations, including those shown in Scheme 1B, might similarly be mimicked under conditions that tolerate long-lived radicals. In this regard, the modern photoredox toolbox will be enabling, as it provides many opportunities for reaction exploration in the late-stages of a synthesis. In turn, this should provide confidence in the early planning stages of design when considering a noncanonical disconnection. Beyond synthetic applications, we believe that a better mechanistic picture of noncanonical oxygenases will emerge from bio-mimicry, including the extent to which these enzymes exert a conformational bias that cannot be accessed by the substrate alone. These secondary 
enzyme-substrate interactions remain difficult to replicate, which helps to explain why radical rearrangement is so uncommon for existing $\mathrm{C}-\mathrm{H}$ oxidation reactions that use an $\mathrm{Fe}=\mathrm{O} .{ }^{41}$ In this regard, we note that it is possible to generate carbon-centered radicals from alcohols under similar conditions to those used here. ${ }^{27}$ Therefore, we see a future opportunity to interface canonical $\mathrm{C}-\mathrm{H}$ hydroxylation reactions with subsequent photoredox catalysis as a means of exploring noncanonical transformations with increasing efficiency and scope.

\section{ACKNOWLEDGMENTS}

We thank Dr. Thierry Maris (University of Montreal) for help with X-ray crystallography, and Professor Nicolas Moitessier and Jessica Plescia for help with ozonolysis. Financial support was provided by the Natural Sciences and Engineering Council (NSERC) of Canada (Discovery Grant to J.-P.L.); and the Fonds de Recherche du Québec Nature et Technologies (FRQNT) (Team Grant to J.-P.L.); and the FRQNT Center for Green Chemistry and Catalysis.

\section{REFERENCES AND NOTES}

1. Ishihara, Y.; Baran, P. S. Two-Phase Terpene Total Synthesis: Historical Perspective and Application to the Taxol ${ }^{\circledR}$ Problem. Synlett 2010, 2010, 1733-1745.

2. Ortiz de Montellano, P. R.; De Voss, J. J. Substrate Oxidation by Cytochrome P450 Enzymes. In Cytochrome P450: Structure, Mechanism, and Biochemistry, Ortiz de Montellano, P. R., Ed. Springer International Publishing: New York, 2015; pp 183-245.

3. Huang, X.; Groves, J. T. Oxygen Activation and Radical Transformations in Heme Proteins and Metalloporphyrins. Chem. Rev. 2018, 118, 2491-2553.

4. Walsh, C. T.; Moore, B. S. Enzymatic Cascade Reactions in Biosynthesis. Angew. Chem. Int. Ed. 2019, 58, 6846-6879.

5. Tang, M.-C.; Zou, Y.; Watanabe, K.; Walsh, C. T.; Tang, Y. Oxidative Cyclization in Natural Product Biosynthesis. Chem. Rev. 2017, 117, 5226-5333.

6. Hu, D. X.; Withall, D. M.; Challis, G. L.; Thomson, R. J. Structure, Chemical Synthesis, and Biosynthesis of Prodiginine Natural Products. Chem. Rev. 2016, 116, 7818-7853.

7. Lau, W.; Sattely, E. S. Six Enzymes from Mayapple that Complete the Biosynthetic Pathway to the Etoposide Aglycone. Science 2015, 349, 1224-1228.

8. Forneris, C. C.; Seyedsayamdost, M. R. In Vitro Reconstitution of OxyC Activity Enables Total Chemoenzymatic Syntheses of Vancomycin Aglycone Variants. Angew. Chem. Int. Ed. 2018, 57, 8048-8052.

9. Böttcher, T. An Additive Definition of Molecular Complexity. J. Chem. Inf. Model. 2016, 56, 462-470.

10. Chang, W.-C.; Yang, Z.--.; Tu, Y.-H.; Chien, T.-C. Reaction Mechanism of a Nonheme Iron Enzyme Catalyzed Oxidative Cyclization via C-C Bond Formation. Org. Lett. 2019, 21, 228-232.

11. Li, J.; Zhang, X.; Renata, H. Asymmetric Chemoenzymatic Synthesis of (-)-Podophyllotoxin and Related Aryltetralin Lignans. Angew. Chem. Int. Ed. 2019, 58, 11657-11660.

12. Li, L.; Chen, Z.; Zhang, X.; Jia, Y. Divergent Strategy in Natural Product Total Synthesis. Chem. Rev. 2018, 118, $3752-$ 3832.

13. Chang, J.; Reiner, J.; Xie, J. Progress on the Chemistry of Dibenzocyclooctadiene Lignans. Chem. Rev. 2005, 105, 45814609.

14. Xu, L.-J.; Peng, Y.; Chen, S.-B.; Chen, S.-L.; Xiao, P.-G. Four New Lignans from Kadsura heteroclita. Heterocycles 2007, 71, 941-947.

15. Lin, Y.-C.; Cheng, Y.-B.; Liaw, C.-C.; Lo, I.-W.; Kuo, Y.-H.; Chiang, M. Y.; Chou, C.-H.; Shen, Y.-C. New Lignans from the Leaves and Stems of Kadsura philippinensis. Molecules 2013, 18, 6573-6583.

16. Shen, Y.-C.; Lin, Y.-C.; Cheng, Y.-B.; Kuo, Y.-H.; Liaw, C.-C. Taiwankadsurins A, B, and C, Three New C19 Homolignans from Kadsura philippinensis. Org. Lett. 2005, 7, 5297-5300. 
17. Roque, J. B.; Kuroda, Y.; Göttemann, L. T.; Sarpong, R. Deconstructive Diversification of Cyclic Amines. Nature 2018, 564, 244-248.

18. Osberger, T. J.; Rogness, D. C.; Kohrt, J. T.; Stepan, A. F.; White, M. C. Oxidative Diversification of Amino Acids and Peptides by Small-Molecule Iron Catalysis. Nature 2016, 537, 214.

19. Yan, M.; Lo, J. C.; Edwards, J. T.; Baran, P. S. Radicals: Reactive Intermediates with Translational Potential. J. Am. Chem. Soc. 2016, 138, 12692-12714.

20. Keylor, M. H.; Matsuura, B. S.; Griesser, M.; Chauvin, J.-P. R.; Harding, R. A.; Kirillova, M. S.; Zhu, X.; Fischer, O. J.; Pratt, D. A.; Stephenson, C. R. J. Synthesis of Resveratrol Tetramers via a Stereoconvergent Radical Equilibrium. Science 2016, 354, 1260-1265.

21. Wertjes, W. C.; Southgate, E. H.; Sarlah, D. Recent Advances in Chemical Dearomatization of Nonactivated Arenes. Chem. Soc. Rev. 2018, 47, 7996-8017.

22. P. Green, S.; A. Whiting, D. New Carbon Radical Chemistry as a Model for the Biogenesis of the Interiorin/Kadsulignan Type of Dibenzocyclooctadiene Lignan. J. Chem. Soc. Perkin Trans. 1 1998, 193-202.

23. Coleman, R. S.; Guernon, J. M.; Roland, J. T. Synthesis of the Spirocyclic Cyclohexadienone Ring System of the Schiarisanrins. Org. Lett. 2000, 2, 277-280.

24. DeMartino, M. P. The Design and Development of Oxidative Enolate Heterocoupling and Application Toward the Total Synthesis of the Taiwankadsurins. PhD dissertation, The Scripps Research Institute, La Jolla, 2008.

25. Barton, D. H. R.; Bridon, D.; Fernandaz-Picot, I.; Zard, S. Z. The invention of radical reactions : Part XV. Some Mechanistic Aspects of the Decarboxylative Rearrangement of Thiohydroxamic Esters. Tetrahedron 1987, 43, 2733-2740. 26. Shaw, M. H.; Twilton, J.; MacMillan, D. W. C. Photoredox Catalysis in Organic Chemistry. J. Org. Chem. 2016, 81, 68986926.

27. Pitre, S. P.; Weires, N. A.; Overman, L. E. Forging C(sp3)-C(sp3) Bonds with Carbon-Centered Radicals in the Synthesis of Complex Molecules. J. Am. Chem. Soc. 2019, 141, 2800-2813.

28. Bringmann, G.; Gulder, T.; Gulder, T. A. M.; Breuning, M. Atroposelective Total Synthesis of Axially Chiral Biaryl Natural Products. Chem. Rev. 2011, 111, 563-639.

29. Coleman, R. S.; Gurrala, S. R.; Mitra, S.; Raao, A. Asymmetric Total Synthesis of Dibenzocyclooctadiene Lignan Natural Products. J. Org. Chem. 2005, 70, 8932-8941.

30. Gong, W.; RajanBabu, T. V. Conformation and Reactivity in Dibenzocyclooctadienes (DBCOD). A General Approach to the Total Synthesis of Fully Substituted DBCOD Lignans via Borostannylative Cyclization of $\alpha, \omega$-diynes. Chem. Sci. 2013, 4, 3979-3985.

31. Belokon, Y. N.; Clegg, W.; Harrington, R. W.; Maleev, V. I.; North, M.; Pujol, M. O.; Usanov, D. L.; Young, C. MechanismGuided Development of VO(salen)X Complexes as Catalysts for the Asymmetric Synthesis of Cyanohydrin Trimethylsilyl Ethers. Chem. Eur. J. 2009, 15, 2148-2165.

32. Bartoli, G.; Bosco, M.; Di Martino, E.; Marcantoni, E.; Sambri, L. Highly Stereoselective and Efficient Addition of Organocerium Reagents to syn- $\beta$-Alkyl- $\beta$-hydroxy- $\alpha$-methyl Ketones by Way of Their Titanium Alkoxides - Synthesis of Complex 1,3-Diol Units with Three Stereodefined Centres. Eur. J. Org. Chem. 2001, 2001, 2901-2909.

33. Huang, Z.; Lumb, J.-P. Phenol-Directed C-H Functionalization. ACS Catal. 2019, 9, 521-555.

34. Kinzel, T.; Zhang, Y.; Buchwald, S. L. A New Palladium Precatalyst Allows for the Fast Suzuki-Miyaura Coupling Reactions of Unstable Polyfluorophenyl and 2-Heteroaryl Boronic Acids. J. Am. Chem. Soc. 2010, 132, 14073-14075.

35. Patel, N. D.; Sieber, J. D.; Tcyrulnikov, S.; Simmons, B. J.; Rivalti, D.; Duvvuri, K.; Zhang, Y.; Gao, D. A.; Fandrick, K. R.; Haddad, N.; Lao, K. S.; Mangunuru, H. P. R.; Biswas, S.; Qu, B.; Grinberg, N.; Pennino, S.; Lee, H.; Song, J. J.; Gupton, B. F.; Garg, N. K.; Kozlowski, M. C.; Senanayake, C. H. Computationally Assisted Mechanistic Investigation and Development of Pd-Catalyzed Asymmetric Suzuki-Miyaura and Negishi Cross-Coupling Reactions for Tetra-ortho-Substituted Biaryl Synthesis. ACS Catal. 2018, 8, 10190-10209. 
36. Wang, G.-Z.; Shang, R.; Fu, Y. Irradiation-Induced Palladium-Catalyzed Decarboxylative Heck Reaction of Aliphatic N(Acyloxy)phthalimides at Room Temperature. Org. Lett. 2018, 20, 888-891.

37. Chuentragool, P.; Kurandina, D.; Gevorgyan, V. Catalysis with Palladium Complexes Photoexcited by Visible Light. Angew. Chem. Int. Ed. 2019, 58, 11586-11598.

38. Smith, J. M.; Harwood, S. J.; Baran, P. S. Radical Retrosynthesis. Acc. Chem. Res. 2018, 51, 1807-1817.

39. Satoshi, I.; Koji, K.; Shigeru, I.; Teruaki, M. A New and Facile Method for the Direct Preparation of $\alpha$-Hydroxycarboxylic Acid Esters from $\alpha, \beta$-Unsaturated Carboxylic Acid Esters with Molecular Oxygen and Phenylsilane Catalyzed by Bis(dipivaloylmethanato)manganese(II) Complex. Chem. Lett. 1990, 19, 1869-1872.

40. Crossley, S. W. M.; Obradors, C.; Martinez, R. M.; Shenvi, R. A. Mn-, Fe-, and Co-Catalyzed Radical Hydrofunctionalizations of Olefins. Chem. Rev. 2016, 116, 8912-9000.

41. Bigi, M. A.; Reed, S. A.; White, M. C. Diverting Non-haem Iron Catalysed Aliphatic C-H Hydroxylations Towards Desaturations. Nat. Chem. 2011, 3, 216. 
Mimicking Noncanonical Oxidations with Redox-Neutral Photocatalysis

\author{
Zheng Huang and Jean-Philip Lumb* \\ Department of Chemistry, McGill University, Montreal, QC H3A 0B8, Canada. \\ *email : jean-philip.lumb@mcgill.ca
}

Supporting Information

\title{
Table of Contents
}

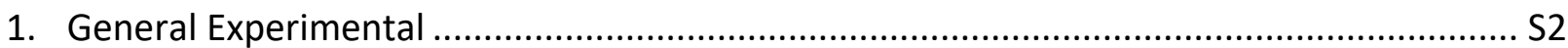

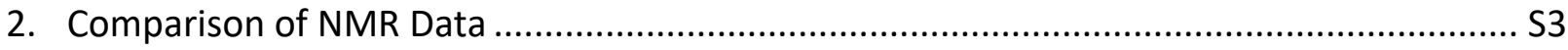

3. Synthesis and Characterization of Synthetic Intermediates........................................ S7

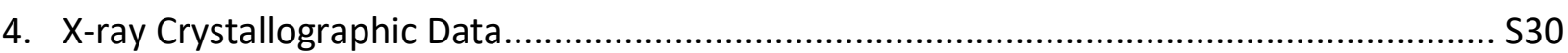

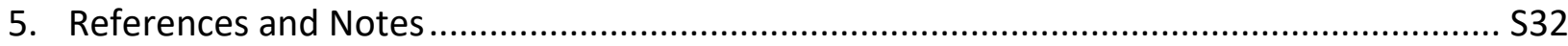




\section{General Experimental}

\section{Purchasing and purification of reagents:}

Chemicals and solvents were purchased from Sigma Aldrich, Alfa Aesar, Strem Chemicals, TCl or Oakwood, and used as received without further purification unless otherwise noted. Solvents were dried and purified using a PureSolv MD 7 (from Innovative Technology) or MB SPS 800 (from MBraun). We have not observed differences in the reaction outcome using either of these solvent purifiers. Unless otherwise noted, solvent was degassed by sparging $\mathrm{N}_{2}$ for $10 \mathrm{~min}$ at $\mathrm{rt}$ prior to use.

\section{Acquisition and analysis of NMR data:}

Proton nuclear magnetic resonance ( ${ }^{1} \mathrm{H}$ NMR) spectra were acquired using Bruker Ascend $500 \mathrm{MHz}$ or $400 \mathrm{MHz}$ spectrometers. Chemical shifts $(\delta)$ are reported in parts per million $(\mathrm{ppm})$ and are calibrated to the residual solvent peak. Coupling constants $(J)$ are reported in $\mathrm{Hz}$. Multiplicities are reported using the following abbreviations: $\mathrm{s}=$ singlet; $\mathrm{d}=$ doublet; $\mathrm{t}=$ triplet; $\mathrm{q}=$ quartet; quint = quintet; $\mathrm{dd}$ = doublet of doublets; $\mathrm{dt}$ = doublet of triplets; $\mathrm{dq}=$ doublet of quartets; $\mathrm{tt}$ = triplet of triplets; $\mathrm{qd}$ = quartet of doublets; qq = quartet of quartets; $\mathrm{ddd}=$ doublet of doublet of doublets; $\mathrm{ddt}=$ doublet of doublet of triplets; dddd = doublet of doublet of doublet of doublets; $b r=b r o a d ; ~ m=$ multiplet (range of multiplet is given). Carbon nuclear magnetic resonance $\left({ }^{13} \mathrm{C}\right.$ NMR) spectra were acquired using Bruker Ascend $126 \mathrm{MHz}$ or $101 \mathrm{MHz}$ spectrometers. Chemical shifts $(\delta)$ are reported in parts per million (ppm) and are calibrated to the residual solvent peak (7.26 for $\mathrm{CHCl}_{3}$ residue in ${ }^{1} \mathrm{H} \mathrm{NMR}$ and 77.16 for $\mathrm{CDCl}_{3}$ in ${ }^{13} \mathrm{C} \mathrm{NMR}$ ).

\section{Additional information:}

High resolution mass spectra (HRMS) were recorded using a Bruker maXis Impact TOF mass spectrometer by electrospray ionization time of flight reflectron experiments. Fourier-transform infrared (FT-IR) spectra were recorded on a Bruker Alpha FT-IR spectrometer. Optical rotations were recorded on a Jasco DIP-140 polarimeter (using the sodium D line; 589 nm). High performance liquid chromatography (HPLC) was measured on an Agilent 1200 Infinity Series HPLC system using a Chiralpak ${ }^{\circledR}$ IC analytical column $(250 \times 4.6 \mathrm{~mm}, 5 \mu \mathrm{m})$. Analytical thin-layer chromatography was performed on pre-coated $250 \mathrm{~mm}$ layer thickness silica gel 60 F254 plates (EMD Chemicals Inc). Visualization was performed by ultraviolet light and/or by staining with potassium permanganate or cerium molybdate. Purifications by column chromatography were performed using standard column chromatography using silica gel (40-63 $\mu \mathrm{m}, 230-400$ mesh). The blue LED light strip (Product Code: CL-FRS5050WPDD-5M-12V-BL) and the 12V, 25W power supply (Product Code: CL-PS94670-25W) for photoredox chemistry was purchased from Creative Lighting Solutions. The LED strip was cut into 2 meters pieces and coiled inside of a $125 \times 65$ crystallization dish wrapped with aluminum foil. 


\section{Comparison of NMR Data}

Table S1. NMR Comparison of heteroclitin J (3).

\begin{tabular}{|c|c|c|c|c|c|}
\hline & \multicolumn{2}{|c|}{${ }^{1} \mathrm{H}-\mathrm{NMR}$} & & \multicolumn{2}{|c|}{${ }^{13}$ C-NMR } \\
\hline & Natural $^{1}$ & Synthetic & & Natural $^{1}$ & Synthetic \\
\hline 4 & $6.52(\mathrm{~s}, 1 \mathrm{H})$ & $6.51(\mathrm{~d}, J=1.2 \mathrm{~Hz}, 1 \mathrm{H})$ & 1 & 196.04 & 196.05 \\
\hline 11 & $6.51(\mathrm{~s}, 1 \mathrm{H})$ & $6.50(\mathrm{~s}, 1 \mathrm{H})$ & 2 & 131.88 & 131.92 \\
\hline 6 & $5.90(\mathrm{~s}, 1 \mathrm{H})$ & $5.89(\mathrm{~d}, J=1.1 \mathrm{~Hz}, 1 \mathrm{H})$ & 3 & 155.61 & 155.71 \\
\hline 9 & $5.89(\mathrm{~s}, 1 \mathrm{H})$ & $5.88(\mathrm{~s}, 1 \mathrm{H})$ & 4 & 123.05 & 123.09 \\
\hline 8 & $2.25(\mathrm{q}, J=7.2 \mathrm{~Hz}, 1 \mathrm{H})$ & $2.24(\mathrm{q}, J=7.2 \mathrm{~Hz}, 1 \mathrm{H})$ & 5 & 141.79 & 141.85 \\
\hline 17 & $1.27(\mathrm{~s}, 3 \mathrm{H})$ & $1.25(\mathrm{~s}, 3 \mathrm{H})$ & 6 & 81.46 & 81.46 \\
\hline 18 & $1.33(\mathrm{~d}, J=7.2 \mathrm{~Hz}, 3 \mathrm{H})$ & $1.31(\mathrm{~d}, J=7.2 \mathrm{~Hz}, 3 \mathrm{H})$ & 7 & 75.18 & 75.21 \\
\hline \multirow{2}{*}{19} & $4.79,4.06$ & $4.68(\mathrm{~d}, J=9.0 \mathrm{~Hz}, 1 \mathrm{H})$ & 8 & 44.13 & 44.20 \\
\hline & $(\mathrm{ABq}, J=8.8 \mathrm{~Hz}, 1 \mathrm{H}$ each $)$ & $4.05(\mathrm{~d}, J=9.0 \mathrm{~Hz}, 1 \mathrm{H})$ & 9 & 82.37 & 82.41 \\
\hline $7-\mathrm{OH}$ & $2.54(\mathrm{br} \mathrm{s}, 1 \mathrm{H})$ & $2.54(\mathrm{~s}, 1 \mathrm{H})$ & 10 & 129.24 & 129.30 \\
\hline $2-\mathrm{OCH}_{3}$ & $3.77(\mathrm{~s}, 3 \mathrm{H})$ & $3.75(\mathrm{~s}, 3 \mathrm{H})$ & 11 & 101.21 & 101.23 \\
\hline $3-\mathrm{OCH}_{3}$ & $4.09(\mathrm{~s}, 3 \mathrm{H})$ & $4.06(\mathrm{~s}, 3 \mathrm{H})$ & 12 & 150.16 & 150.19 \\
\hline \multirow{2}{*}{20} & $5.95,5.91$ & $5.93(\mathrm{~d}, J=1.4 \mathrm{~Hz}, 1 \mathrm{H})$ & 13 & 130.42 & 130.44 \\
\hline & (brs-like, $1 \mathrm{H}$ each) & $5.88(\mathrm{~d}, J=1.5 \mathrm{~Hz}, 1 \mathrm{H})$ & 14 & 143.43 & 143.47 \\
\hline Ac & $1.88(\mathrm{~s}, 3 \mathrm{H})$ & $1.86(\mathrm{~s}, 3 \mathrm{H})$ & 15 & 120.29 & 120.35 \\
\hline \multirow{3}{*}{$\mathrm{Bz}$} & $7.34(\mathrm{~d}, J=7.2 \mathrm{~Hz}, 2 \mathrm{H})$ & $7.32(\mathrm{~d}, J=7.5 \mathrm{~Hz}, 2 \mathrm{H})$ & 16 & 63.25 & 63.28 \\
\hline & $7.30(\mathrm{t}, J=8 \mathrm{~Hz}, 2 \mathrm{H})$ & $7.29(\mathrm{t}, J=7.7 \mathrm{~Hz}, 2 \mathrm{H})$ & 17 & 28.41 & 28.47 \\
\hline & $7.53(\mathrm{t}, J=7.2 \mathrm{~Hz}, 1 \mathrm{H})$ & $7.51(\mathrm{tt}, J=7.1,1.6 \mathrm{~Hz}, 1 \mathrm{H})$ & 18 & 17.75 & 17.77 \\
\hline & \multirow{11}{*}{ OMe } & & 19 & 77.35 & 78.87 \\
\hline & & & 20 & 101.98 & 102.02 \\
\hline & & & $2-\mathrm{OCH}_{3}$ & 58.94 & 58.97 \\
\hline & & & $3-\mathrm{OCH}_{3}$ & 58.89 & 58.92 \\
\hline & & & 1 & 168.62 & 168.68 \\
\hline & & & ne & 20.35 & 20.39 \\
\hline & & & & 165.09 & 165.12 \\
\hline & & & & 128.31 & 128.35 \\
\hline & & & $\mathrm{Bz}$ & 129.74 & 129.75 \\
\hline & & & & 128.17 & 128.21 \\
\hline & & & & 133.88 & 133.91 \\
\hline
\end{tabular}


Table S2. NMR Comparison of kadsuphilin N (4).

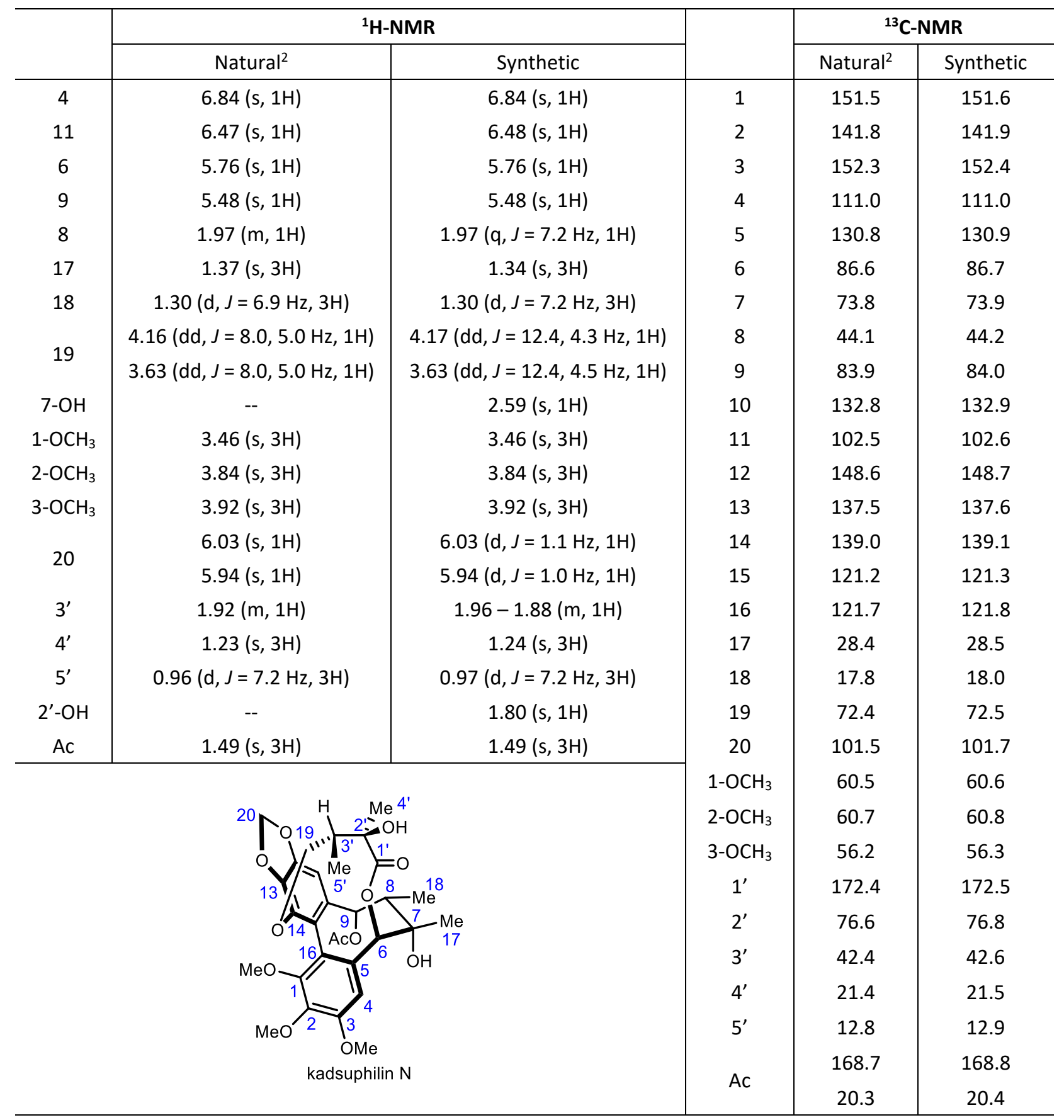


Table S3. NMR Comparison of taiwankadsurin A (4a).

\begin{tabular}{|c|c|c|c|c|c|}
\hline & \multicolumn{2}{|c|}{${ }^{1} \mathrm{H}-\mathrm{NMR}$} & & \multicolumn{2}{|c|}{${ }^{13}$ C-NMR } \\
\hline & Natural $^{3}$ & Synthetic & & Natural $^{3}$ & Synthetic \\
\hline 4 & $6.08(\mathrm{~d}, J=2.7 \mathrm{~Hz}, 1 \mathrm{H})$ & $6.08(\mathrm{~d}, J=2.7 \mathrm{~Hz}, 1 \mathrm{H})$ & 1 & $97.6 \mathrm{~s}$ & 97.7 \\
\hline 11 & $6.60(\mathrm{~s}, 1 \mathrm{H})$ & $6.60(\mathrm{~s}, 1 \mathrm{H})$ & 2 & $171.1 \mathrm{~s}$ & 171.1 \\
\hline 6 & $6.49(\mathrm{~d}, J=2.7 \mathrm{~Hz}, 1 \mathrm{H})$ & $6.49(\mathrm{~d}, J=2.7 \mathrm{~Hz}, 1 \mathrm{H})$ & 3 & $165.5 \mathrm{~s}$ & 165.5 \\
\hline 9 & $6.69(\mathrm{~d}, J=3.0 \mathrm{~Hz}, 1 \mathrm{H})$ & $6.69(\mathrm{~d}, J=3.0 \mathrm{~Hz}, 1 \mathrm{H})$ & 4 & $117.3 \mathrm{~d}$ & 117.4 \\
\hline 8 & $2.36(\mathrm{~m}, 1 \mathrm{H})$ & $2.37(\mathrm{qd}, J=6.8,3.3 \mathrm{~Hz}, 1 \mathrm{H})$ & 5 & $150.4 \mathrm{~s}$ & 150.5 \\
\hline 17 & $1.34(\mathrm{~s}, 3 \mathrm{H})$ & $1.34(\mathrm{~s}, 3 \mathrm{H})$ & 6 & $73.3 \mathrm{~d}$ & 73.4 \\
\hline 18 & $1.05(\mathrm{~d}, J=6.9 \mathrm{~Hz}, 3 \mathrm{H})$ & $1.05(\mathrm{~d}, J=6.8 \mathrm{~Hz}, 3 \mathrm{H})$ & 7 & $79.3 \mathrm{~s}$ & 79.4 \\
\hline \multirow{2}{*}{19} & $5.00(\mathrm{~d}, J=10.5 \mathrm{~Hz}, 1 \mathrm{H})$ & $5.00(\mathrm{~d}, J=10.2 \mathrm{~Hz}, 1 \mathrm{H})$ & 8 & $45.3 d$ & 45.3 \\
\hline & $4.54(\mathrm{~d}, J=10.5 \mathrm{~Hz}, 1 \mathrm{H})$ & $4.54(\mathrm{~d}, J=10.2 \mathrm{~Hz}, 1 \mathrm{H})$ & 9 & $70.5 d$ & 70.6 \\
\hline $1-\mathrm{OH}$ & -- & $3.51(\mathrm{~s}, 1 \mathrm{H})$ & 10 & $127.8 \mathrm{~s}$ & 127.9 \\
\hline $2-\mathrm{OCH}_{3}$ & $3.96(\mathrm{~s}, 3 \mathrm{H})$ & $3.96(\mathrm{~s}, 3 \mathrm{H})$ & 11 & $99.1 d$ & 99.1 \\
\hline $3-\mathrm{OCH}_{3}$ & $3.57(\mathrm{~s}, 3 \mathrm{H})$ & $3.57(\mathrm{~s}, 3 \mathrm{H})$ & 12 & $150.3 \mathrm{~s}$ & 150.4 \\
\hline \multirow{2}{*}{20} & $6.02(\mathrm{~s}, 1 \mathrm{H})$ & $6.02(\mathrm{~d}, J=1.4 \mathrm{~Hz}, 1 \mathrm{H})$ & 13 & $129.0 \mathrm{~s}$ & 129.5 \\
\hline & $5.99(\mathrm{~s}, 1 \mathrm{H})$ & $5.99(\mathrm{~d}, J=1.4 \mathrm{~Hz}, 1 \mathrm{H})$ & 14 & $144.6 \mathrm{~s}$ & 144.3 \\
\hline Ac & $2.21(\mathrm{~s}, 3 \mathrm{H})$ & $2.21(\mathrm{~s}, 3 \mathrm{H})$ & 15 & $118.2 \mathrm{~s}$ & 118.2 \\
\hline \multirow{3}{*}{$\mathrm{Bz}$} & $8.31(\mathrm{~d}, J=7.5 \mathrm{~Hz}, 2 \mathrm{H})$ & $8.31(\mathrm{~d}, J=7.2 \mathrm{~Hz}, 2 \mathrm{H})$ & 16 & $57.1 \mathrm{~s}$ & 57.1 \\
\hline & $7.53(\mathrm{t}, J=7.5 \mathrm{~Hz}, 2 \mathrm{H})$ & $7.53(\mathrm{t}, J=7.8 \mathrm{~Hz}, 2 \mathrm{H})$ & 17 & $28.3 q$ & 28.4 \\
\hline & $7.65(\mathrm{t}, J=7.5 \mathrm{~Hz}, 1 \mathrm{H})$ & $7.65(t, J=7.4 \mathrm{~Hz}, 1 \mathrm{H})$ & 18 & $8.8 \mathrm{q}$ & 8.9 \\
\hline & \multirow{11}{*}{\multicolumn{2}{|c|}{ (12) }} & 19 & $80.5 \mathrm{t}$ & 80.6 \\
\hline & & & 20 & $101.9 \mathrm{t}$ & 102.0 \\
\hline & & & $2-\mathrm{OCH}_{3}$ & $53.7 \mathrm{q}$ & 53.8 \\
\hline & & & $3-\mathrm{OCH}_{3}$ & $51.8 \mathrm{q}$ & 51.9 \\
\hline & & & 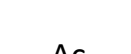 & $169.1 \mathrm{~s}$ & 169.2 \\
\hline & & & $A C$ & $21.3 \mathrm{q}$ & 21.4 \\
\hline & & & & $165.5 \mathrm{~s}$ & 165.7 \\
\hline & & & & $128.4 \mathrm{~s}$ & 128.5 \\
\hline & & & $\mathrm{Bz}$ & $128.9 \mathrm{~d}$ & 129.0 \\
\hline & & & & $130.6 \mathrm{~d}$ & 130.7 \\
\hline & & & & $134.1 \mathrm{~d}$ & 134.2 \\
\hline
\end{tabular}


Table S4. NMR Comparison of taiwankadsurin B (4b).

\begin{tabular}{|c|c|c|c|c|c|}
\hline & \multicolumn{2}{|c|}{${ }^{1} \mathrm{H}-\mathrm{NMR}$} & & \multicolumn{2}{|c|}{${ }^{13}$ C-NMR } \\
\hline & Natural $^{3}$ & Synthetic & & Natural $^{3}$ & Synthetic \\
\hline 4 & $6.17(\mathrm{~d}, J=2.7 \mathrm{~Hz}, 1 \mathrm{H})$ & $6.17(\mathrm{~d}, J=2.7 \mathrm{~Hz}, 1 \mathrm{H})$ & 1 & $97.8 \mathrm{~s}$ & 97.9 \\
\hline 11 & $6.60(s, 1 H)$ & $6.60(s, 1 H)$ & 2 & $170.2 \mathrm{~s}$ & 170.3 \\
\hline 6 & $6.91(\mathrm{~d}, J=2.7 \mathrm{~Hz}, 1 \mathrm{H})$ & $6.90(\mathrm{~d}, J=2.7 \mathrm{~Hz}, 1 \mathrm{H})$ & 3 & $165.7 \mathrm{~s}$ & 165.8 \\
\hline 9 & $6.80(\mathrm{~d}, J=3.0 \mathrm{~Hz}, 1 \mathrm{H})$ & $6.79(\mathrm{~d}, J=3.0 \mathrm{~Hz}, 1 \mathrm{H})$ & 4 & $118.5 \mathrm{~d}$ & 118.6 \\
\hline 8 & $2.38(\mathrm{~m}, 1 \mathrm{H})$ & $2.39(\mathrm{qd}, J=6.8,3.3 \mathrm{~Hz}, 1 \mathrm{H})$ & 5 & $149.6 \mathrm{~s}$ & 149.7 \\
\hline 17 & $1.37(\mathrm{~s}, 3 \mathrm{H})$ & $1.38(\mathrm{~s}, 3 \mathrm{H})$ & 6 & $73.4 \mathrm{~d}$ & 73.5 \\
\hline 18 & $1.04(\mathrm{~d}, J=6.6 \mathrm{~Hz}, 3 \mathrm{H})$ & $1.04(\mathrm{~d}, J=6.8 \mathrm{~Hz}, 3 \mathrm{H})$ & 7 & $78.6 \mathrm{~s}$ & 78.71 \\
\hline \multirow{2}{*}{19} & $4.98(\mathrm{~d}, J=9.9 \mathrm{~Hz}, 1 \mathrm{H})$ & $4.98(\mathrm{~d}, J=10.0 \mathrm{~Hz}, 1 \mathrm{H})$ & 8 & $45.4 d$ & 45.5 \\
\hline & $4.63(\mathrm{~d}, J=9.9 \mathrm{~Hz}, 1 \mathrm{H})$ & $4.63(\mathrm{~d}, J=10.0 \mathrm{~Hz}, 1 \mathrm{H})$ & 9 & $70.5 d$ & 70.5 \\
\hline $1-\mathrm{OH}$ & -- & $4.86(\mathrm{~s}, 1 \mathrm{H})$ & 10 & $127.8 \mathrm{~s}$ & 127.9 \\
\hline $2-\mathrm{OCH}_{3}$ & $3.61(s, 3 H)$ & $3.61(s, 3 H)$ & 11 & $99.4 d$ & 99.5 \\
\hline $3-\mathrm{OCH}_{3}$ & $3.56(\mathrm{~s}, 3 \mathrm{H})$ & $3.56(\mathrm{~s}, 3 \mathrm{H})$ & 12 & $149.9 \mathrm{~s}$ & 150.0 \\
\hline \multirow{2}{*}{20} & $5.96(s, 1 H)$ & $5.96(\mathrm{~d}, J=1.3 \mathrm{~Hz}, 1 \mathrm{H})$ & 13 & $128.8 \mathrm{~s}$ & 129.2 \\
\hline & $5.95(\mathrm{~s}, 1 \mathrm{H})$ & $5.95(\mathrm{~d}, J=1.4 \mathrm{~Hz}, 1 \mathrm{H})$ & 14 & $142.5 \mathrm{~s}$ & 142.8 \\
\hline Ac & $2.21(\mathrm{~s}, 3 \mathrm{H})$ & $2.21(\mathrm{~s}, 3 \mathrm{H})$ & 15 & $120.7 \mathrm{~s}$ & 120.8 \\
\hline \multirow{3}{*}{$\mathrm{Bz}$} & $8.32(\mathrm{~d}, J=7.2 \mathrm{~Hz}, 2 \mathrm{H})$ & $8.32(\mathrm{~d}, J=7.2 \mathrm{~Hz}, 2 \mathrm{H})$ & 16 & $58.8 \mathrm{~s}$ & 58.9 \\
\hline & $7.52(\mathrm{t}, J=7.2 \mathrm{~Hz}, 2 \mathrm{H})$ & $7.53(\mathrm{t}, J=7.7 \mathrm{~Hz}, 2 \mathrm{H})$ & 17 & $28.6 q$ & 28.8 \\
\hline & $7.64(\mathrm{t}, J=7.2 \mathrm{~Hz}, 1 \mathrm{H})$ & $7.64(\mathrm{t}, J=7.4 \mathrm{~Hz}, 1 \mathrm{H})$ & 18 & $8.5 q$ & 8.6 \\
\hline & \multirow{11}{*}{\multicolumn{2}{|c|}{ (12) }} & 19 & $78.6 \mathrm{t}$ & 78.69 \\
\hline & & & 20 & $101.7 \mathrm{t}$ & 101.8 \\
\hline & & & $2-\mathrm{OCH}_{3}$ & $54.1 \mathrm{q}$ & 54.3 \\
\hline & & & $3-\mathrm{OCH}_{3}$ & $51.8 \mathrm{q}$ & 51.9 \\
\hline & & & $\Delta r$ & $169.1 \mathrm{~s}$ & 169.3 \\
\hline & & & $A C$ & $21.3 \mathrm{q}$ & 21.4 \\
\hline & & & & $165.6 \mathrm{~s}$ & 165.8 \\
\hline & & & & $130.0 \mathrm{~s}$ & 128.8 \\
\hline & & & $\mathrm{Bz}$ & $128.8 \mathrm{~d}$ & 128.9 \\
\hline & & & & $130.6 \mathrm{~d}$ & 130.7 \\
\hline & & & & $133.9 \mathrm{~d}$ & 134.0 \\
\hline
\end{tabular}




\section{Synthesis and Characterization of Synthetic Intermediates}

\section{Compound 22}

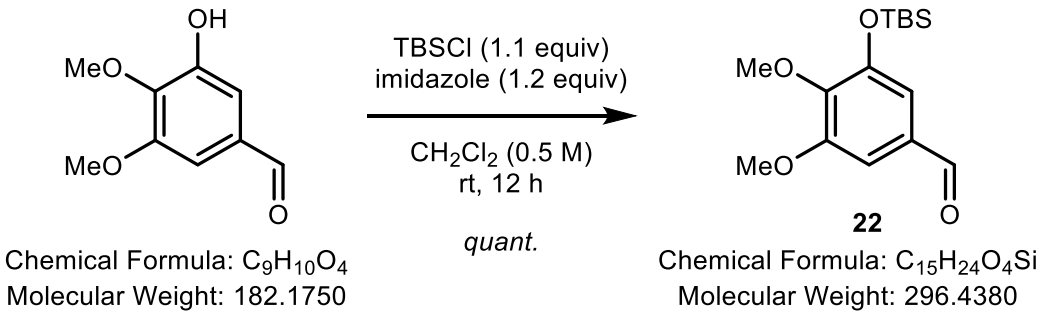

Procedure: A $250 \mathrm{~mL}$, flame-dried round bottom flask equipped with a Teflon-coated stir bar and a rubber septum was charged with 3,4-dimethoxy-5-hydroxybenzaldehyde ( $50 \mathrm{mmol}, 9.11 \mathrm{~g}, 1.0$ equiv), imidazole ( $60 \mathrm{mmol}, 4.08 \mathrm{~g}, 1.2 \mathrm{equiv}$ ) and $\mathrm{TBSCl}$ (55 mmol, $8.29 \mathrm{~g}, 1.1$ equiv). $\mathrm{CH}_{2} \mathrm{Cl}_{2}(100 \mathrm{~mL})$ was then added, and the resulting mixture was stirred at rt for 12 h. It was then poured on $\mathrm{HCl}(1 \mathrm{M}, 100 \mathrm{~mL})$, and extracted with $\mathrm{CH}_{2} \mathrm{Cl}_{2}(3 \times 100 \mathrm{~mL})$. The combined organic layers were dried over $\mathrm{MgSO}_{4}$, filtered and concentrated in vacuo. The crude product was then purified on silica gel (20:1 hexanes/EtOAc) to provide 22 (14.82 g, 50.0 mmol, quant.) as a pale-yellow oil.

\section{Characterization:}

${ }^{1} \mathrm{H}$ NMR $\left(500 \mathrm{MHz}, \mathrm{CDCl}_{3}\right) \delta 9.82(\mathrm{~s}, 1 \mathrm{H}), 7.11(\mathrm{~d}, J=1.8 \mathrm{~Hz}, 1 \mathrm{H}), 7.02(\mathrm{~d}, J=1.8 \mathrm{~Hz}, 1 \mathrm{H}), 3.92(\mathrm{~s}, 3 \mathrm{H}), 3.87(\mathrm{~s}, 3 \mathrm{H}), 1.02(\mathrm{~s}$, $9 \mathrm{H}), 0.21$ (s, 6H); ${ }^{13} \mathrm{C}$ NMR $\left(126 \mathrm{MHz} \mathrm{CDCl}_{3}\right) \delta 191.2,154.4,150.0,146.3,131.9,117.4,105.3,60.7,56.3,25.8,18.5,-4.5$. Our characterization data matches the data reported previously. ${ }^{4}$

\section{Compound SI-1}<smiles>COc1cc(C=O)cc([SeH])c1OC</smiles>

Chemical Formula: $\mathrm{C}_{15} \mathrm{H}_{24} \mathrm{O}_{4} \mathrm{Si}$ Molecular Weight: 296.4380

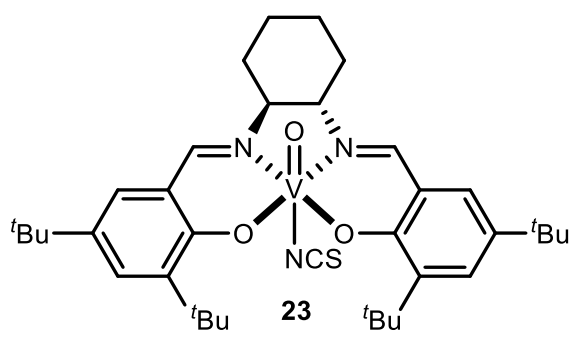

$(0.1 \mathrm{~mol} \%)$

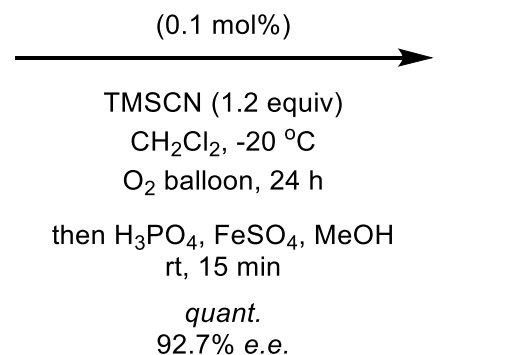

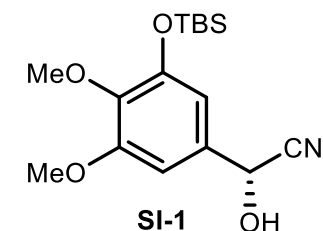

Chemical Formula: $\mathrm{C}_{16} \mathrm{H}_{25} \mathrm{NO}_{4} \mathrm{Si}$ Molecular Weight: 323.4640

Procedure: The literature procedure was followed with slight modifications. ${ }^{5}$ A $100 \mathrm{~mL}$ flame-dried round bottom flask equipped with a Teflon-coated stir bar and a rubber septum was charged with (S,S)-VO(salen)NCS (23) (31) (0.03 mmol, $20.1 \mathrm{mg}, 0.1 \mathrm{~mol} \%$ ) and 22 ( $30 \mathrm{mmol}, 8.89 \mathrm{~g}, 1.0$ equiv). It was then evacuated and refilled with $\mathrm{O}_{2}$ three times before a balloon of $\mathrm{O}_{2}$ was left connected to the flask by a needle inserted into the septum. $\mathrm{CH}_{2} \mathrm{Cl}_{2}(30 \mathrm{~mL})$, which had been sparged with $\mathrm{O}_{2}$ for $10 \mathrm{~min}$ was then added to afford a homogeneous solution (dark green). The resulting homogeneous solution was then cooled to $-20^{\circ} \mathrm{C}$, at which point TMSCN (36 mmol, $4.50 \mathrm{~mL}, 1.2$ equiv) was added dropwise by syringe. The resulting mixture was then placed in a $-20{ }^{\circ} \mathrm{C}$ freezer for $24 \mathrm{~h}$. The reaction mixture was then quenched at this temperature by the addition of a solution consisting of $\mathrm{FeSO}_{4} \cdot 7 \mathrm{H}_{2} \mathrm{O}(3 \mathrm{mmol}, 834.2 \mathrm{mg})$ and $\mathrm{H}_{3} \mathrm{PO}_{4}(12 \mathrm{mmol}, 85 \mathrm{wt} \%$ aq. $0.82 \mathrm{~mL}$ ) in $\mathrm{MeOH}(6 \mathrm{~mL})$, and stirred for 15 min while the reaction mixture gradually warmed to rt. It was then poured on brine $(50 \mathrm{~mL})$ and extracted with $\mathrm{CH}_{2} \mathrm{Cl}_{2}(3 \times 50 \mathrm{~mL})$. The combined organic layers were dried over $\mathrm{MgSO}_{4}$, filtered and concentrated in vacuo. The crude product was then purified on silica gel (3:1 hexanes/EtOAc) to provide SI-1 ( $9.70 \mathrm{~g}, 30.0$ 
mmol, quant.) as a pale-green oil with an e.e. of $92.7 \%$ as determined by chiral HPLC.

\section{Characterization:}

$\mathbf{R}_{f}=$ (hexanes/EtOAc 3:1): 0.40; IR (neat) $v=3427,2931,2858,1589,1500,1455,1426,1344,1252,1229,1114 \mathrm{~cm}^{-1} ;{ }^{1} \mathbf{H}$ NMR $\left(500 \mathrm{MHz}, \mathrm{CDCl}_{3}\right) \delta 6.69(\mathrm{~d}, J=2.1 \mathrm{~Hz}, 1 \mathrm{H}), 6.64(\mathrm{~d}, J=2.0 \mathrm{~Hz}, 1 \mathrm{H}), 5.39(\mathrm{~d}, J=6.7 \mathrm{~Hz}, 1 \mathrm{H}), 3.84(\mathrm{~s}, 3 \mathrm{H}), 3.82(\mathrm{~d}, J=$ $6.7 \mathrm{~Hz}, 1 \mathrm{H}), 3.77(\mathrm{~s}, 3 \mathrm{H}), 0.99(\mathrm{~s}, 9 \mathrm{H}), 0.18(\mathrm{~s}, 6 \mathrm{H}) ;{ }^{13} \mathrm{C}$ NMR $\left(126 \mathrm{MHz}, \mathrm{CDCl}_{3}\right) \delta 154.2,149.8,141.2,130.9,119.0,112.6$, 103.8, 63.3, 60.6, 56.2, 25.7, 18.4, -4.5; HPLC (Chiralpak ${ }^{\circledR}$ IC analytical column, eluent 5:95 ${ }^{i} \mathrm{PrOH}:$ hexanes, $1 \mathrm{~mL} / \mathrm{min}$ ) $\mathrm{t}_{\mathrm{R}}$ 9.7 min (major), 8.1 min (minor), $92.7 \%$ e.e.; $[\alpha]^{23} \mathrm{D}+20.1^{\circ},\left(\mathrm{c}=1.0, \mathrm{CHCl}_{3}\right)$; HRMS: Calcd. for $\mathrm{C}_{16} \mathrm{H}_{25} \mathrm{NO}_{4} \mathrm{Si}[\mathrm{M}+\mathrm{Na}]^{+}=$ $346.1445 \mathrm{~m} / \mathrm{z}$, found $=346.1440 \mathrm{~m} / \mathrm{z}$.

HPLC trace of the racemic mixture:

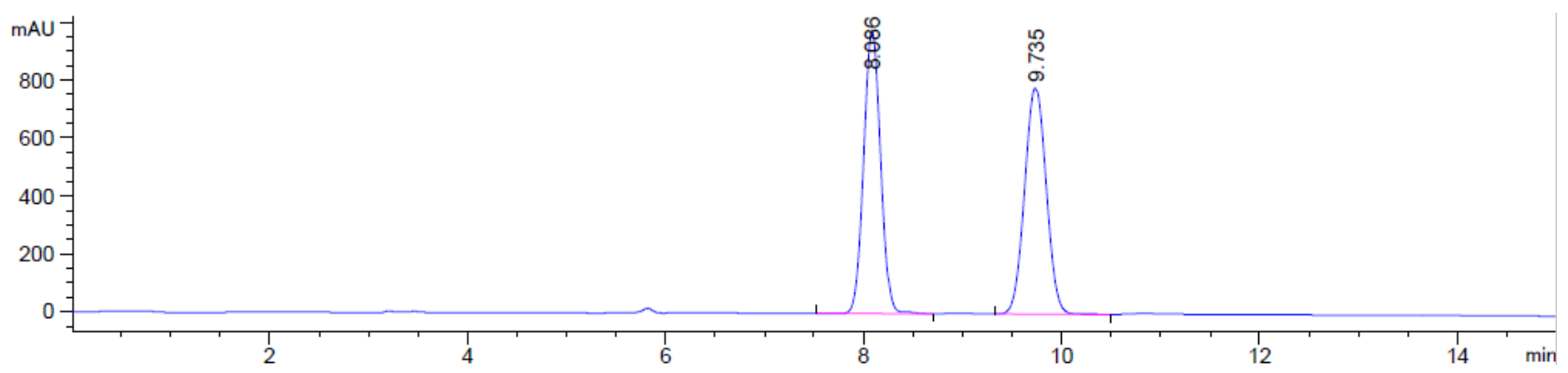

Signal 1: DAD1 A, Sig=209,2 Ref=off

\begin{tabular}{|c|c|c|c|c|c|c|}
\hline $\begin{array}{c}\text { Peak } \\
\#\end{array}$ & $\begin{array}{c}\text { RetTime } \\
\text { [min] }\end{array}$ & Type & $\begin{array}{l}\text { Width } \\
\text { [min] }\end{array}$ & $\begin{array}{c}\text { Area } \\
{\left[\mathrm{mAU}^{*} \mathrm{~s}\right]}\end{array}$ & $\begin{array}{c}\text { Height } \\
\text { [mAU] }\end{array}$ & $\begin{array}{c}\text { Area } \\
\&\end{array}$ \\
\hline & . & & ------ & --------- & ------- & $------\mid$ \\
\hline 1 & 8.086 & BB & 0.1871 & $1.16411 \mathrm{e} 4$ & 976.22260 & 49.9088 \\
\hline 2 & 9.735 & VB & 0.2346 & $1.16836 \mathrm{e} 4$ & 780.71277 & 50.0912 \\
\hline
\end{tabular}

\section{HPLC trace of the chiral product:}

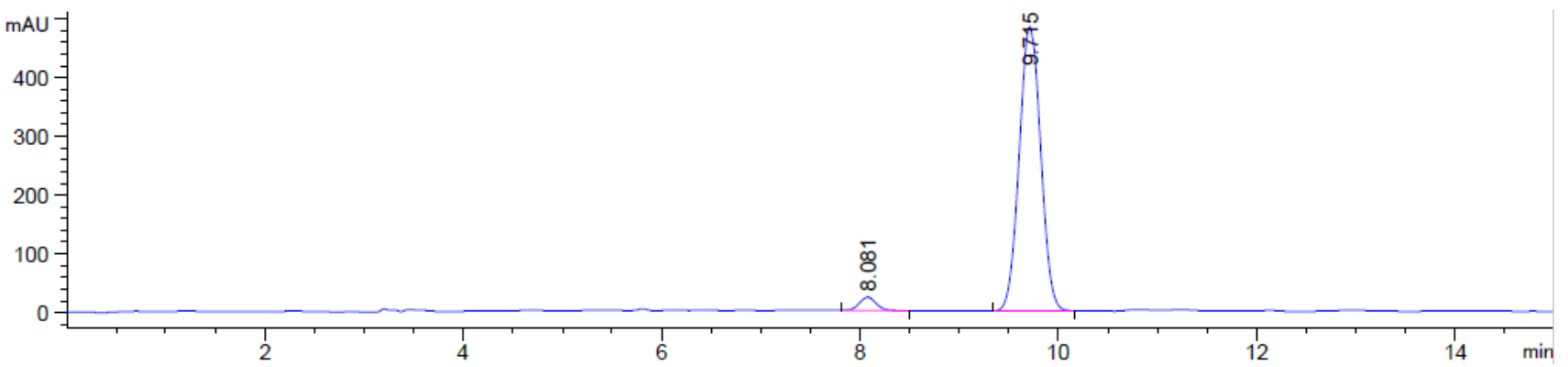

Signal 1: DAD1 A, Sig=209,2 Ref=off

\begin{tabular}{|c|c|c|c|c|c|c|}
\hline $\begin{array}{c}\text { Peak } \\
\#\end{array}$ & $\begin{array}{c}\text { RetTime } \\
\text { [min] }\end{array}$ & Type & $\begin{array}{l}\text { Width } \\
\text { [min] }\end{array}$ & $\begin{array}{c}\text { Area } \\
{\left[\mathrm{mAU}^{*} \mathrm{~s}\right]}\end{array}$ & $\begin{array}{l}\text { Height } \\
\text { [mAU] }\end{array}$ & $\begin{array}{c}\text { Area } \\
\&\end{array}$ \\
\hline--1 & $\mid----0$ & & --- & --------- & --------- & $--------\mid$ \\
\hline 1 & 8.081 & BB & 0.1882 & 271.15393 & 22.56488 & 3.6460 \\
\hline 2 & 9.715 & $\mathrm{BB}$ & 0.2329 & 7165.92236 & 483.49017 & 96.3540 \\
\hline
\end{tabular}




\section{Compound SI-2}
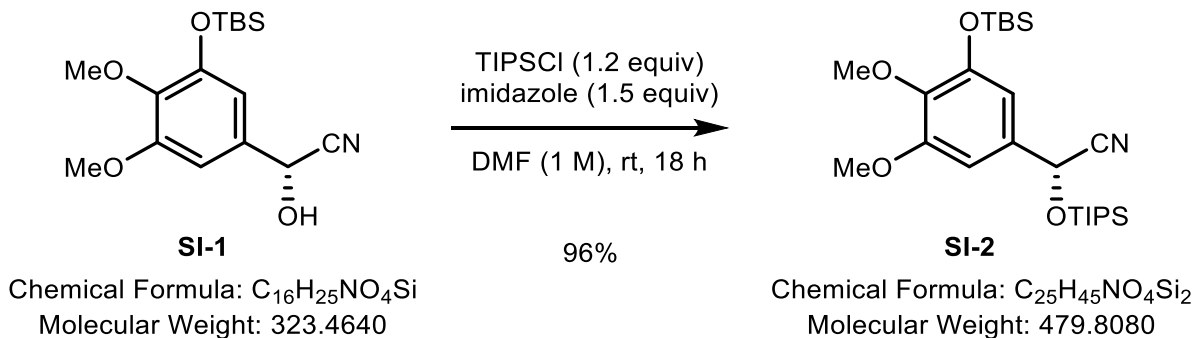

Procedure: A $100 \mathrm{~mL}$, flame-dried round bottom flask equipped with a Teflon-coated stir bar and a rubber septum was charged with SI-1 (30 mmol, $9.70 \mathrm{~g}, 1.0$ equiv) and DMF (30 mL). TIPSCI (36 mol, $7.70 \mathrm{~mL}, 1.2$ equiv) was then added via syringe, followed by the addition of imidazole ( $45 \mathrm{mmol}, 3.06 \mathrm{~g}, 1.5$ equiv) as a solid. The resulting solution was then stirred at $\mathrm{rt}$ for $18 \mathrm{~h}$, and then quenched by the addition of $\mathrm{N}, \mathrm{N}$-dimethylaminoethanol $(1.20 \mathrm{~mL})$, and stirred for another $1 \mathrm{~h}$. It was then diluted with EtOAc $(100 \mathrm{~mL})$, and washed with $\mathrm{HCl}(1 \mathrm{M}, 50 \mathrm{~mL})$. The aqueous layer was further extracted with EtOAc $(2 \times 100 \mathrm{~mL})$. The combined organic layers were washed with water $(100 \mathrm{~mL})$ and brine $(100 \mathrm{~mL})$, dried over $\mathrm{MgSO}_{4}$, filtered and concentrated in vacuo. The crude product was then purified on silica gel (20:1 hexanes/EtOAc) to provide SI-2 (13.75 g, $28.65 \mathrm{mmol}, 96 \%)$ as a colorless oil.

\section{Characterization:}

$\mathbf{R}_{f}=$ (hexanes/EtOAc 10:1): 0.57; IR (neat) $\mathbf{v}=2942,2866,1589,1500,1462,1426,1346,1251,1224,1146,1108 \mathrm{~cm}^{-1} ;{ }^{1} \mathbf{H}$ NMR $\left(500 \mathrm{MHz}_{2} \mathrm{CDCl}_{3}\right) \delta 6.68(\mathrm{~d}, J=2.0 \mathrm{~Hz}, 1 \mathrm{H}), 6.62(\mathrm{~d}, J=1.7 \mathrm{~Hz}, 1 \mathrm{H}), 5.47(\mathrm{~s}, 1 \mathrm{H}), 3.86(\mathrm{~s}, 3 \mathrm{H}), 3.79(\mathrm{~s}, 3 \mathrm{H}), 1.25-1.17$ $(\mathrm{m}, 3 \mathrm{H}), 1.11(\mathrm{~d}, J=7.3 \mathrm{~Hz}, 9 \mathrm{H}), 1.07(\mathrm{~d}, J=7.3 \mathrm{~Hz}, 9 \mathrm{H}), 1.00(\mathrm{~s}, 9 \mathrm{H}), 0.17(\mathrm{~s}, 6 \mathrm{H}) ;{ }^{13} \mathrm{C} \mathrm{NMR}(126 \mathrm{MHz}, \mathrm{CDCl}) \delta 154.2,149.8$, $141.1,132.2,119.5,112.0,103.1,64.0,60.5,56.1,25.8,18.4,17.93,17.92,12.1,-4.6 ;[\alpha]^{23}{ }_{\mathrm{D}}+3.8^{\circ},\left(\mathrm{c}=1.0, \mathrm{CHCl}_{3}\right)$; HRMS: Calcd. for $\mathrm{C}_{25} \mathrm{H}_{45} \mathrm{NO}_{4} \mathrm{Si}_{2}[\mathrm{M}+\mathrm{Na}]^{+}=502.2779 \mathrm{~m} / \mathrm{z}$, found $=502.2788 \mathrm{~m} / \mathrm{z}$.

\section{Compound 24}

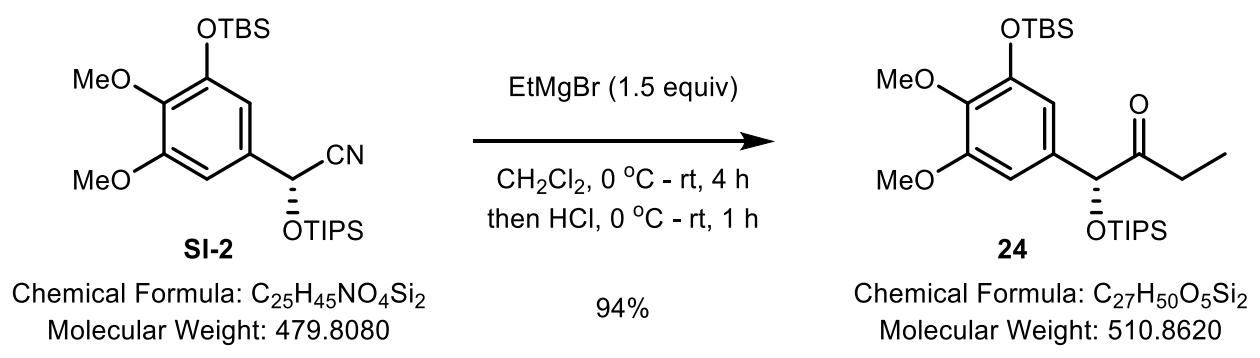

Procedure: A $500 \mathrm{~mL}$ flame-dried round bottom flask equipped with a Teflon-coated stir bar and a rubber septum was charged with SI-2 (33.28 mmol, $16.79 \mathrm{~g}, 1.0$ equiv). The flask was evacuated and refilled with $\mathrm{N}_{2}$ three times, prior to the addition of $\mathrm{CH}_{2} \mathrm{Cl}_{2}(140 \mathrm{~mL})$. The resulting solution was then cooled to $0^{\circ} \mathrm{C}$ in an ice bath, at which point $\mathrm{EtMgBr}(50.0$ mmol, $3 \mathrm{M}$ in Et $\mathrm{E}_{2} \mathrm{O}, 16.67 \mathrm{~mL}, 1.5$ equiv) was added dropwise via syringe. The solution was then warmed to rt and stirred for $4 \mathrm{~h}$, and then cooled to $0{ }^{\circ} \mathrm{C}$ again in an ice bath. The reaction mixture was then quenched by the addition of $\mathrm{HCl}(2 \mathrm{M}$, $140 \mathrm{~mL}$ ), and stirred for an additional $1 \mathrm{~h}$ at rt. The biphasic mixture was then extracted with $\mathrm{CH}_{2} \mathrm{Cl}_{2}(3 \times 100 \mathrm{~mL})$. The combined organic layers were dried over $\mathrm{MgSO}_{4}$, filtered and concentrated in vacuo. The crude product was then purified on silica gel (20:1 hexanes/EtOAc) to provide 24 (15.98 g, $31.28 \mathrm{mmol}, 94 \%)$ as a pale-yellow oil.

\section{Characterization:}

$\mathbf{R}_{\boldsymbol{f}}=$ (hexanes/EtOAc 10:1): 0.64; IR (neat) $\mathbf{v}=2940,2866,1717,1586,1498,1462,1423,1389,1343,1251,1235,1221$ $1115 \mathrm{~cm}^{-1} ;{ }^{1} \mathrm{H}$ NMR $\left(500 \mathrm{MHz}, \mathrm{CDCl}_{3}\right) \delta 6.66(\mathrm{~d}, J=1.9 \mathrm{~Hz}, 1 \mathrm{H}), 6.61(\mathrm{~d}, J=1.8 \mathrm{~Hz}, 1 \mathrm{H}), 5.05(\mathrm{~s}, 1 \mathrm{H}), 3.82(\mathrm{~s}, 3 \mathrm{H}), 3.76(\mathrm{~s}$, $3 \mathrm{H}), 2.69(\mathrm{dq}, J=18.6,7.3 \mathrm{~Hz}, 1 \mathrm{H}), 2.45(\mathrm{dq}, J=18.6,7.3 \mathrm{~Hz}, 1 \mathrm{H}), 1.16-1.10(\mathrm{~m}, 3 \mathrm{H}), 1.05-1.01(\mathrm{~m}, 18 \mathrm{H}), 1.00(\mathrm{~s}, 9 \mathrm{H})$, $0.93(\mathrm{t}, J=7.3 \mathrm{~Hz}, 3 \mathrm{H}), 0.16(\mathrm{~s}, 3 \mathrm{H}), 0.15(\mathrm{~s}, 3 \mathrm{H}) ;{ }^{13} \mathrm{C}$ NMR $\left(126 \mathrm{MHz}, \mathrm{CDCl}_{3}\right) \delta 211.5,153.8,149.5,140.1,134.6,111.6$, 
103.0, 81.3, 60.5, 56.1, 28.5, 25.8, 18.5, 18.0, 12.2, 7.6, -4.5, -4.6; $[\alpha]^{23}{ }_{\mathrm{D}}+33.8^{\circ},\left(\mathrm{c}=1.0, \mathrm{CHCl}_{3}\right)$; HRMS: Calcd. for $\mathrm{C}_{27} \mathrm{H}_{50} \mathrm{O}_{5} \mathrm{Si}_{2}[\mathrm{M}+\mathrm{Na}]^{+}=533.3089 \mathrm{~m} / \mathrm{z}$, found $=533.3081 \mathrm{~m} / \mathrm{z}$.

\section{Compound 26}<smiles>O=Cc1cc(O)c2c(c1)OCO2</smiles>

Chemical Formula: $\mathrm{C}_{8} \mathrm{H}_{6} \mathrm{O}_{4}$ Molecular Weight: 166.1320

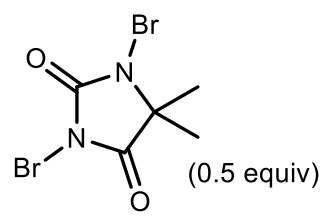

$\mathrm{CHCl}_{3}(0.1 \mathrm{M}), \mathrm{rt}, 12 \mathrm{~h}$

then MOMCl (2.0 equiv) DIPEA (2.4 equiv), rt, $12 \mathrm{~h}$

$75 \%$<smiles>COc1c(Br)c(C=O)cc2c1OCO2</smiles>

Chemical Formula: $\mathrm{C}_{10} \mathrm{H}_{9} \mathrm{BrO}_{5}$ Molecular Weight: 289.0810

Procedure: A 1 L oven-dried round bottom flask equipped with a Teflon-coated stir bar and a rubber septum was charged with 5-hydroxypiperonal ( $50 \mathrm{mmol}, 8.31 \mathrm{~g}, 1.0$ equiv) and $\mathrm{CHCl}_{3}(500 \mathrm{~mL})$. 1,3-Dibromo-5,5-dimethylhydantoin (DBDMH, $25 \mathrm{~mol}, 7.15 \mathrm{~g}, 0.5$ equiv) was added in portions (an orange color appeared when the first portion of DBDMH was added, the next portion was added after the orange color faint). The resulting mixture was stirred at $\mathrm{rt}$ for $12 \mathrm{~h}$. $N, N$ Diisopropylethylamine (DIPEA, $120 \mathrm{mmol}, 20.90 \mathrm{~mL}, 2.4$ equiv) and $\mathrm{MOMCl}$ (100 mmol, $7.60 \mathrm{~mL}, 2.0$ equiv) was then added via syringe, and the mixture was stirred for another $12 \mathrm{~h}$ at rt. It was then poured on $\mathrm{HCl}(1 \mathrm{M}, 200 \mathrm{~mL})$, and extracted with $\mathrm{CH}_{2} \mathrm{Cl}_{2}(3 \times 200 \mathrm{~mL})$. The combined organic layers were dried over $\mathrm{MgSO}_{4}$, filtered and concentrated in vacuo. The crude product was then purified on silica gel (10:1 to 5:1 hexanes/EtOAc) to provide 26 (10.87 g, $37.60 \mathrm{mmol}$, $75 \%)$ as a white solid.

\section{Characterization:}

$\mathbf{R}_{f}=$ (hexanes/EtOAc 5:1): 0.32; IR (neat) $v=2949,2880,1666,1595,1475,1464,1403,1380,1343,1315,1281,1222$, 1153, $1132 \mathrm{~cm}^{-1} ;{ }^{1}{ }^{H}$ NMR (500 MHz, CDCl 3 ) $\delta 10.26(\mathrm{~s}, 1 \mathrm{H}), 7.20(\mathrm{~s}, 1 \mathrm{H}), 6.09(\mathrm{~s}, 2 \mathrm{H}), 5.33(\mathrm{~s}, 2 \mathrm{H}), 3.61(\mathrm{~s}, 3 \mathrm{H}) ;{ }^{13} \mathrm{C} \mathrm{NMR}$ $\left(126 \mathrm{MHz}_{2} \mathrm{CDCl}_{3}\right) \delta 190.7,149.2,144.0,137.1,128.9,116.7,104.0,103.0,97.4,57.8$; HRMS: Calcd. for $\mathrm{C}_{10} \mathrm{H}_{9} \mathrm{BrO}_{5}[\mathrm{M}+\mathrm{Na}]^{+}$ $=310.9526 \mathrm{~m} / \mathrm{z}$, found $=310.9525 \mathrm{~m} / \mathrm{z}$. 


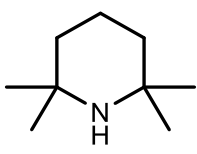

$42 \mathrm{mmol}$

Chemical Formula: $\mathrm{C}_{9} \mathrm{H}_{19} \mathrm{~N}$ Molecular Weight: 141.2580

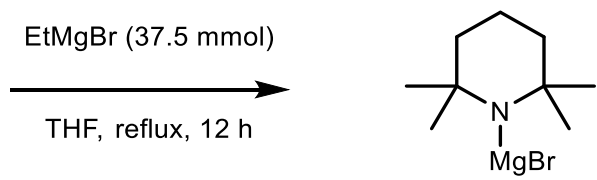

Chemical Formula: $\mathrm{C}_{9} \mathrm{H}_{18} \mathrm{BrMgN}$ Molecular Weight: 244.4590<smiles>CCCCCCC(=O)Oc1cc([C@@H](O[SnH])C(=O)CC)cc(OC)c1OC</smiles>

$24,25 \mathrm{mmol}$

Chemical Formula: $\mathrm{C}_{27} \mathrm{H}_{50} \mathrm{O}_{5} \mathrm{Si}_{2}$ Molecular Weight: 510.8620

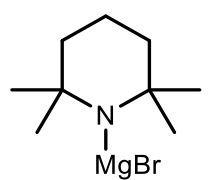

$\operatorname{THF}(0.2 \mathrm{M})$

$-78{ }^{\circ} \mathrm{C}, 1 \mathrm{~h}$

then $\mathrm{Cy}_{2} \mathrm{BCl}$

(1.2 equiv) $\mathrm{rt}, 1 \mathrm{~h}$

Procedure: $T M P M g B r$ was made following the literature procedure. ${ }^{6} \mathrm{~A} 500 \mathrm{~mL}$ flame-dried round bottom flask equipped with a Teflon-coated stir bar and a rubber septum was charged with 2,2,6,6-tetramethylpiperidine (freshly distilled over $\mathrm{CaH}_{2}, 42 \mathrm{mmol}, 7.15 \mathrm{~mL}$ ) and dissolved in THF (37.5 mL). EtMgBr (37.5 mmol, $12.5 \mathrm{~mL}$ of $3 \mathrm{M}$ solution in Et $\left.{ }_{2} \mathrm{O}, 1.5 \mathrm{equiv}\right)$ was then added via syringe. The resulting solution was then refluxed under $\mathrm{N}_{2}$ for $12 \mathrm{~h}$, dilute with additional THF (37.5 $\mathrm{mL}$ ) and cooled to $-78{ }^{\circ} \mathrm{C}$ in a dry ice / acetone bath. A solution of 24 ( $25 \mathrm{mmol}, 12.77 \mathrm{~g}, 1.0$ equiv) in THF (50 mL) was then added dropwise over $30 \mathrm{~min}$ with a syringe pump. The deprotonation was complete within another $1 \mathrm{~h}$ at $-78^{\circ} \mathrm{C}$, at which point a solution of $\mathrm{Cy}_{2} \mathrm{BCl}(30 \mathrm{mmol}, 1.2$ equiv) in hexane $(30 \mathrm{~mL})$ was added via syringe, warm to rt and stirred for another $1 \mathrm{~h}$. It was then cooled down again to $-78^{\circ} \mathrm{C}$, and a solution of $\mathbf{2 6}$ ( $25 \mathrm{mmol}, 7.23 \mathrm{~g}, 1.0$ equiv) in hexanes and $\mathrm{CH}_{2} \mathrm{Cl}_{2}(1: 1$ mixture, $75 \mathrm{~mL})$ was added dropwise over $30 \mathrm{~min}$ with a syringe pump. The resulting mixture was then stirred at $-78{ }^{\circ} \mathrm{C}$ for $3 \mathrm{~h}$, and additional $2 \mathrm{~h}$ at $-30{ }^{\circ} \mathrm{C}$. It was quenched by the addition of $\mathrm{NaHCO}_{3}$ (saturated, $100 \mathrm{~mL}$ ) and diethanolamine $(75 \mathrm{mmol}, 7.89 \mathrm{~g}, 3.0$ equiv), and stirred for another $30 \mathrm{~min}$ at $\mathrm{rt}$. The mixture was then filtered, and the filtrate was extracted with EtOAc $(3 \times 200 \mathrm{~mL})$. The combined organic layers were dried over $\mathrm{MgSO}_{4}$, filtered and concentrated in vacuo. The crude product was then purified on silica gel (10:1 to 5:1 hexanes/EtOAc) to provide 27 (15.80 g, $19.75 \mathrm{mmol}, 79 \%$ ) as a 20:1 d.r. mixture. To further purify the product, it was then dissolved in hexanes (150 mL) with gentle heat, cooled to $\mathrm{rt}$, and left in a $-20^{\circ} \mathrm{C}$ freezer for $12 \mathrm{~h}$. The resulting white solid was collected by filtration. The process was repeated with $100 \mathrm{~mL}$ of hexanes, and gave $13.20 \mathrm{~g}, 16.50 \mathrm{mmol}, 66 \%$ of 27 as a white solid with > 99\% e.e. as determined by chiral HPLC.

\section{Characterization:}

$\mathbf{R}_{f}=$ (hexanes/EtOAc 5:1): 0.32; IR (neat) $v=3453,2937,2864,1708,1585,1497,1471,1425,1390,1355,1334,1280$, 1248, 1222, 1158, 1148, $1109 \mathrm{~cm}^{-1} ;{ }^{1} \mathrm{H}$ NMR $\left(500 \mathrm{MHz}, \mathrm{CDCl}_{3}\right) \delta 6.66(\mathrm{~s}, 1 \mathrm{H}), 6.54(\mathrm{~d}, J=1.7 \mathrm{~Hz}, 1 \mathrm{H}), 6.52(\mathrm{~d}, J=1.8 \mathrm{~Hz}$ $1 \mathrm{H}), 5.95(\mathrm{~d}, J=1.5 \mathrm{~Hz}, 1 \mathrm{H}), 5.95(\mathrm{~d}, J=1.5 \mathrm{~Hz}, 1 \mathrm{H}), 5.29(\mathrm{~d}, J=6.0 \mathrm{~Hz}, 1 \mathrm{H}), 5.15(\mathrm{~d}, J=6.0 \mathrm{~Hz}, 1 \mathrm{H}), 5.08$ (dd, $J=7.1,6.0$ $\mathrm{Hz}, 1 \mathrm{H}), 5.02(\mathrm{~s}, 1 \mathrm{H}), 3.90(\mathrm{~d}, J=7.2 \mathrm{~Hz}, 1 \mathrm{H}), 3.79(\mathrm{~s}, 3 \mathrm{H}), 3.76(\mathrm{~s}, 3 \mathrm{H}), 3.53(\mathrm{~s}, 3 \mathrm{H}), 3.49(\mathrm{qd}, J=7.2,6.0 \mathrm{~Hz}, 1 \mathrm{H}), 1.08-1.03$ (m, 3H), $1.01-0.96(\mathrm{~m}, 30 \mathrm{H}), 0.13(\mathrm{~s}, 6 \mathrm{H}) ;{ }^{13} \mathrm{C}$ NMR $\left(126 \mathrm{MHz} \mathrm{CDCl}_{3}\right) \delta 214.6,153.7,149.4,148.7,140.2,137.5,136.4$, 136.3, 133.6, 112.4, 108.5, 103.6, 103.0, 101.9, 97.1, 80.6, 75.6, 60.5, 57.5, 56.0, 45.6, 25.8, 18.4, 17.98, 17.96, 16.1, 12.2, -4.58, -4.62; HPLC (Chiralpak ${ }^{\circledR}$ IC analytical column, eluent 10:90 ${ }^{i}$ PrOH:hexanes, $1 \mathrm{~mL} / \mathrm{min}$ ) $\mathrm{t}_{\mathrm{R}} 7.9 \mathrm{~min}$ (major), $10.8 \mathrm{~min}$ (minor), > 99\% e.e.; $[\alpha]^{23}{ }_{\mathrm{D}}-17.2^{\circ}$, $\left(\mathrm{c}=1.0, \mathrm{CHCl}_{3}\right)$; HRMS: Calcd. for $\mathrm{C}_{37} \mathrm{H}_{59} \mathrm{BrO}_{10} \mathrm{Si}_{2}[\mathrm{M}+\mathrm{Na}]^{+}=821.2722 \mathrm{~m} / \mathrm{z}$, found $=$ $821.2729 \mathrm{~m} / \mathrm{z}$. 
HPLC trace of the racemic mixture:

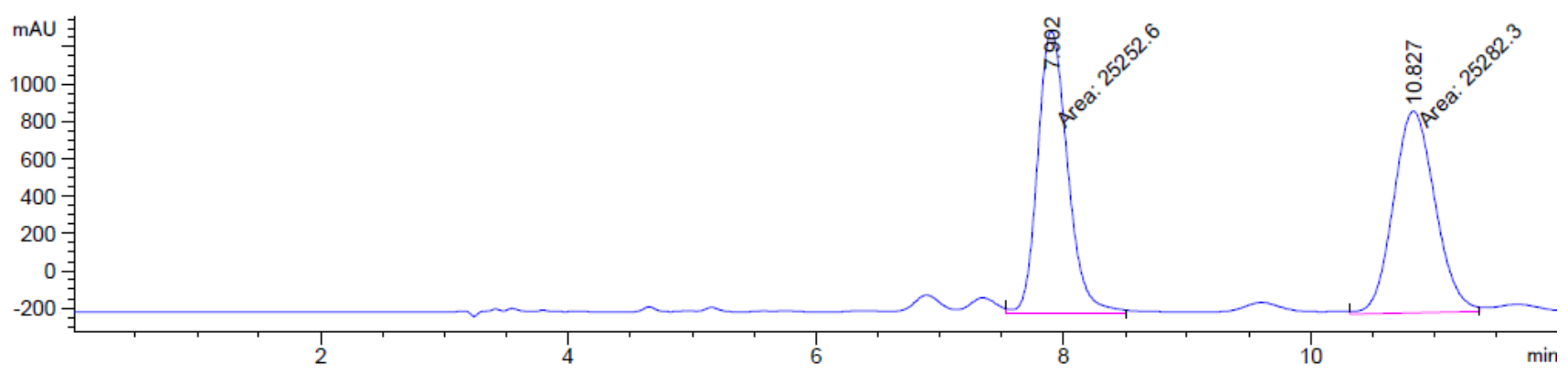

Signal 1: DAD1 A, Sig=218,2 Ref=off

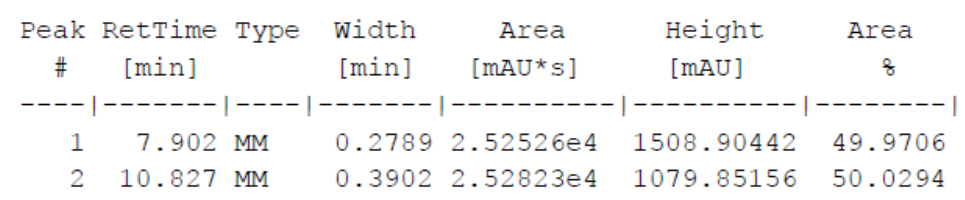

\section{HPLC trace of the chiral product:}

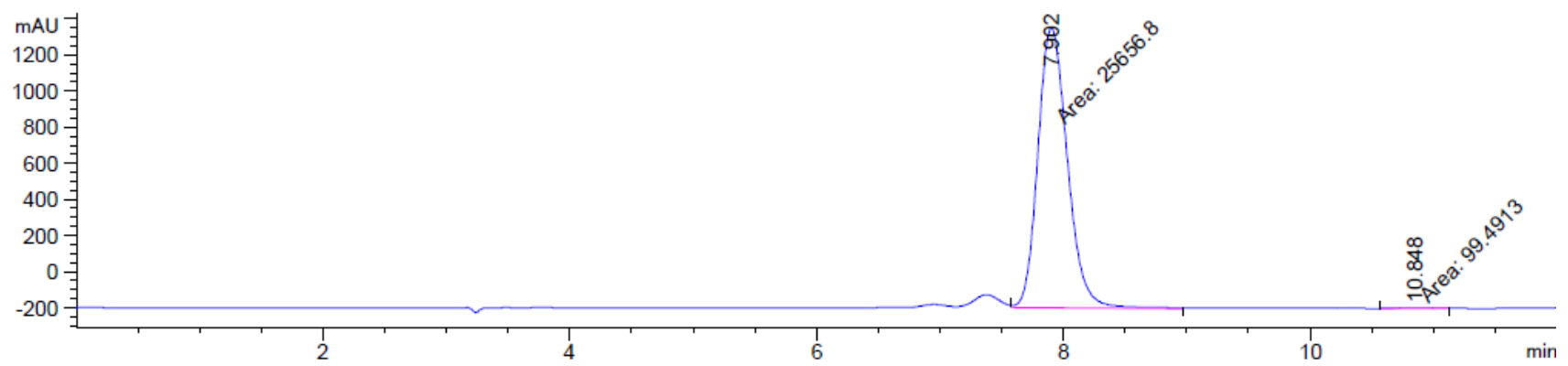

Signal 1: DAD1 A, Sig=218,2 Ref=off

\begin{tabular}{|c|c|c|c|c|c|c|}
\hline $\begin{array}{c}\text { Peak } \\
\quad \#\end{array}$ & $\begin{array}{c}\text { RetTime } \\
\text { [min] }\end{array}$ & Type & $\begin{array}{l}\text { Width } \\
{[\mathrm{min}]}\end{array}$ & $\begin{array}{c}\text { Area } \\
{\left[\mathrm{mAU}^{*} \mathrm{~s}\right]}\end{array}$ & $\begin{array}{l}\text { Height } \\
\text { [mAU] }\end{array}$ & $\begin{array}{c}\text { Area } \\
\&\end{array}$ \\
\hline & & & & -- & 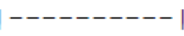 & ------ \\
\hline 1 & 7.902 & MM & 0.2763 & $2.56568 \mathrm{e} 4$ & 1547.61865 & 99.6137 \\
\hline 2 & 10.848 & MM & 0.3296 & 99.49126 & 5.03051 & 0.3863 \\
\hline
\end{tabular}

\section{Compound 28}<smiles>COc1cc([C@H](O[AsH3])C(=O)[C@@H](C)[C@H](O)c2cc3c(c(OC)c2Br)OCO3)cc(OC)c1OC</smiles>

Chemical Formula: $\mathrm{C}_{37} \mathrm{H}_{59} \mathrm{BrO}_{10} \mathrm{Si}_{2}$ Molecular Weight: 799.9430

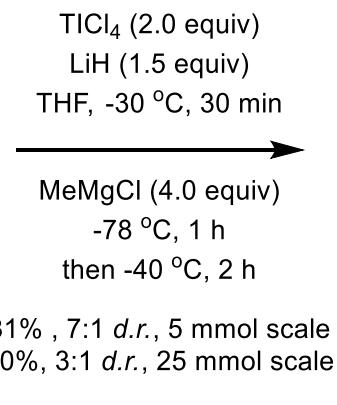

Chemical Formula: $\mathrm{C}_{38} \mathrm{H}_{63} \mathrm{BrO}_{10} \mathrm{Si}_{2}$ Molecular Weight: 815.9860

Procedure: The literature procedure was followed with slight modifications. ${ }^{7}$ A $100 \mathrm{~mL}$ flame-dried round bottom flask equipped with a Teflon-coated stir bar and a rubber septum was charged with 27 (5.0 mmol, $4.00 \mathrm{~g}, 1.0$ equiv) and LiH ( $7.5 \mathrm{mmol}, 59.6 \mathrm{mg}, 1.5$ equiv). The flask was evacuated and refill with $\mathrm{N}_{2}$ (repeat 3 times), prior to the addition of THF (25 mL). The mixture was then cooled to $-30{ }^{\circ} \mathrm{C}$ in a dry ice bath, at which point $\mathrm{TiCl}_{4}\left(10.0 \mathrm{mmol}, 1 \mathrm{M} \mathrm{in} \mathrm{CH}_{2} \mathrm{Cl}_{2}, 10.0 \mathrm{~mL}\right.$, 2.0 equiv) was added dropwise via syringe, and stirred at this temperature for $30 \mathrm{~min}$. The resulting red solution was then 
cooled to $-78^{\circ} \mathrm{C}$, a solution of $\mathrm{MeMgCl}(20 \mathrm{mmol}, 1 \mathrm{M}$ solution in THF, $20 \mathrm{~mL}, 4.0$ equiv) was added dropwise over $30 \mathrm{~min}$ with a syringe pump. The mixture was stirred at this temperature for $1 \mathrm{~h}$, warmed to $-40{ }^{\circ} \mathrm{C}$ and stirred for another $2 \mathrm{~h}$. It was then quenched by the addition of a solution containing $\mathrm{NH}_{4} \mathrm{Cl}$ (saturated, $20 \mathrm{~mL}$ ) and $\mathrm{NH}_{4} \mathrm{OH}$ (concentrated, $5 \mathrm{~mL}$ ), and stirred for another $15 \mathrm{~min}$ at $\mathrm{rt}$. The resulting mixture was then filtered, and the filtrate was extracted with EtOAc ( 3 $\times 50 \mathrm{~mL}$ ). The combined organic layers were dried over $\mathrm{MgSO}_{4}$, filtered and concentrated in vacuo. The crude product was then purified on silica gel (5:1 to 3:1 hexanes/EtOAc) to provide the major diastereomer (28) (3.31 g, $4.06 \mathrm{mmol}, 81 \%)$ as a pale-yellow oil, and the minor diastereomer $(453.8 \mathrm{mg}, 0.56 \mathrm{mmol}, 11 \%)$ as a pale-yellow oil. A similar experiment with $25 \mathrm{mmol}$ of $\mathbf{2 7}$ gave $14.33 \mathrm{~g}, 17.6 \mathrm{mmol}, 70 \%$ of $\mathbf{2 8}$ and 3:1 diastereomeric ratio.

\section{Characterization:}

Major diastereomer (28): $\mathbf{R}_{f}=$ (hexanes/EtOAc 5:1): 0.21; IR (neat) v = 3441, 2938, 2865, 1585, 1500, 1470, 1426, 1386, 1350, 1290, 1272, 1225, 1160, 1145, $1115 \mathrm{~cm}^{-1}$; ${ }^{1} \mathrm{H}$ NMR $\left(500 \mathrm{MHz}, \mathrm{CDCl}_{3}\right) \delta 6.72$ (br s, 1H), 6.55 (s, $\left.1 \mathrm{H}\right), 6.43$ (br s, $\left.1 \mathrm{H}\right)$, $5.91(\mathrm{~d}, J=1.4 \mathrm{~Hz}, 1 \mathrm{H}), 5.90(\mathrm{~d}, J=1.4 \mathrm{~Hz}, 1 \mathrm{H}), 5.35(\mathrm{~d}, J=9.0 \mathrm{~Hz}, 1 \mathrm{H}), 5.27(\mathrm{~d}, J=6.0 \mathrm{~Hz}, 1 \mathrm{H}), 5.21(\mathrm{~d}, J=6.0 \mathrm{~Hz}, 1 \mathrm{H}), 5.13$ $(\mathrm{s}, 1 \mathrm{H}), 4.52(\mathrm{~s}, 1 \mathrm{H}), 3.82(\mathrm{~s}, 3 \mathrm{H}), 3.80(\mathrm{~s}, 3 \mathrm{H}), 3.56(\mathrm{~s}, 1 \mathrm{H}), 3.54(\mathrm{~s}, 3 \mathrm{H}), 1.54(\mathrm{~s}, 3 \mathrm{H}), 1.55-1.45(\mathrm{~m}, 1 \mathrm{H}), 1.14-0.77(\mathrm{~m}$, $30 \mathrm{H}), 0.46$ (d, $J=7.1 \mathrm{~Hz}, 3 \mathrm{H}), 0.15$ (br s, 6H); ${ }^{13} \mathrm{C}$ NMR $\left(126 \mathrm{MHz} \mathrm{CDCl}_{3}\right) \delta 153.4,148.9,140.5,137.7,137.1,135.9,135.2$, 115.0, 109.9, 106.2, 103.7, 101.8, 97.2, 80.5, 79.9, 74.5, 60.6, 57.5, 56.2, 45.1, 25.8, 21.6, 18.5, 18.3, 18.1, 12.7, 12.3, 4.6; $[\alpha]^{23}{ }_{D}+13.9^{\circ},\left(c=1.0, \mathrm{CHCl}_{3}\right)$; HRMS: Calcd. for $\mathrm{C}_{38} \mathrm{H}_{63} \mathrm{BrO}_{10} \mathrm{Si}_{2}[\mathrm{M}+\mathrm{Na}]^{+}=837.3035 \mathrm{~m} / \mathrm{z}$, found $=837.3028 \mathrm{~m} / \mathrm{z}$.

Minor diastereomer: $\mathbf{R}_{f}=$ (hexanes/EtOAc 5:1): 0.32; IR (neat) $v=3377,2938,2864,1585,1499,1469,1426,1388,1354$, 1331, 1249, 1223, 1160, $1114 \mathrm{~cm}^{-1} ;{ }^{1} \mathrm{H}$ NMR (500 MHz, CDCl $) \delta 6.82(\mathrm{~s}, 1 \mathrm{H}), 6.75(\mathrm{~d}, J=1.2 \mathrm{~Hz}, 1 \mathrm{H}), 6.68(\mathrm{~d}, J=1.7 \mathrm{~Hz}$, $1 \mathrm{H}), 5.94(\mathrm{~s}, 2 \mathrm{H}), 5.34(\mathrm{~d}, J=9.8 \mathrm{~Hz}, 1 \mathrm{H}), 5.26(\mathrm{~d}, J=6.0 \mathrm{~Hz}, 1 \mathrm{H}), 5.22(\mathrm{~d}, J=6.0 \mathrm{~Hz}, 1 \mathrm{H}), 5.19(\mathrm{~s}, 1 \mathrm{H}), 5.04(\mathrm{br} \mathrm{s}, 1 \mathrm{H}), 3.83$ $(\mathrm{s}, 3 \mathrm{H}), 3.75(\mathrm{~s}, 3 \mathrm{H}), 3.53(\mathrm{~s}, 3 \mathrm{H}), 3.40(\mathrm{br} \mathrm{s}, 1 \mathrm{H}), 2.11(\mathrm{dq}, J=9.8,7.3 \mathrm{~Hz}, 1 \mathrm{H}), 1.28(\mathrm{~s}, 3 \mathrm{H}), 1.05-0.96(\mathrm{~m}, 30 \mathrm{H}), 0.43(\mathrm{~d}, J$ $=7.4 \mathrm{~Hz}, 3 \mathrm{H}), 0.15(\mathrm{~s}, 3 \mathrm{H}), 0.12(\mathrm{~s}, 3 \mathrm{H}) ;{ }^{13} \mathrm{C}$ NMR $\left(126 \mathrm{MHz} \mathrm{CDCl}_{3}\right) \delta 153.2,148.9,148.8,140.4,138.6,137.7,136.3,135.9$, $115.5,109.8,106.4,103.5,101.9,97.2,79.7,79.2,75.7,60.6,57.5,56.1,47.3,25.8,22.0,18.4,18.2,18.1,12.7,12.5$, 4.6, $-4.7 ;[\alpha]^{23}{ }_{\mathrm{D}}-23.6^{\circ},\left(\mathrm{c}=1.0, \mathrm{CHCl}_{3}\right)$; HRMS: Calcd. for $\mathrm{C}_{38} \mathrm{H}_{63} \mathrm{BrO}_{10} \mathrm{Si}_{2}[\mathrm{M}+\mathrm{Na}]^{+}=837.3035 \mathrm{~m} / \mathrm{z}$, found $=837.3041 \mathrm{~m} / \mathrm{z}$.

\section{Compound SI-3}
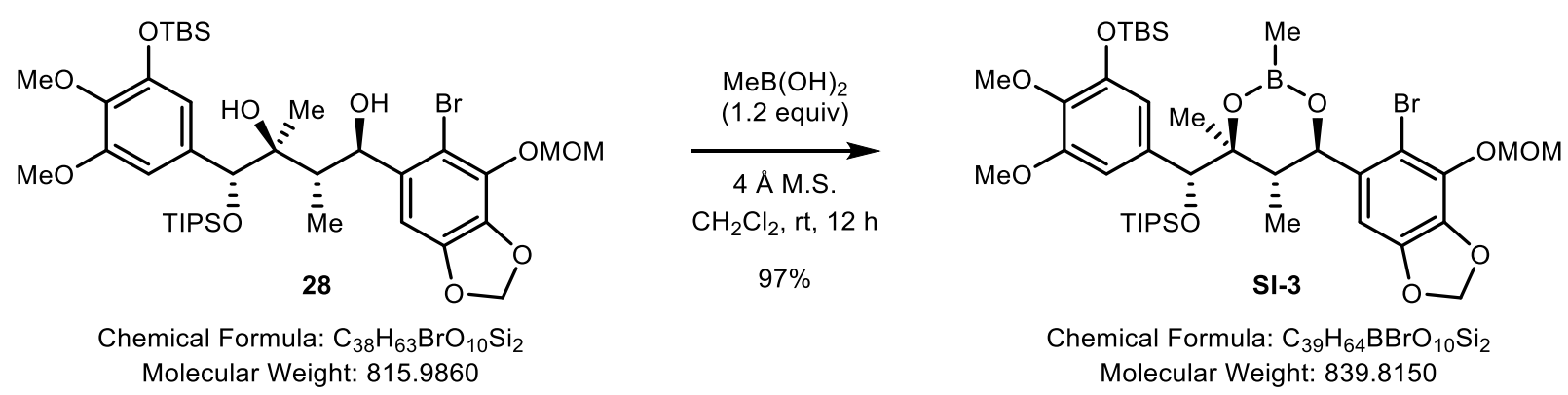

Procedure: A $250 \mathrm{~mL}$ flame-dried round bottom flask equipped with a Teflon-coated stir bar and a rubber septum was charged with 28 (20.0 mmol, $16.32 \mathrm{~g}, 1.0$ equiv), $\mathrm{MeB}(\mathrm{OH})_{2}\left(24 \mathrm{mmol}, 1.44 \mathrm{~g}, 1.2\right.$ equiv) and $4 \AA \mathrm{Aol}^{\mathrm{M}}$ Sieve $(10 \mathrm{~g}) . \mathrm{CH}_{2} \mathrm{Cl}$ $(100 \mathrm{~mL})$ was then added, and the resulting mixture was stirred at $\mathrm{rt}$ for $12 \mathrm{~h}$. It was then filtered and concentrated in vacuo. The crude product was purified on silica gel (10:1 hexanes/EtOAc) to provide SI-3 (16.35 g, 19.46 mmol, $97 \%$ ) as a pale-yellow oil.

\section{Characterization:}

$\mathbf{R}_{\boldsymbol{f}}=$ (hexanes/EtOAc 10:1): 0.37; IR (neat) $v=2938,2865,1585,1499,1471,1425,1404,1383,1364,1335,1273,1239$, 1224, $1159 \mathrm{~cm}^{-1}$; ${ }^{1} \mathrm{H}$ NMR $\left(500 \mathrm{MHz}, \mathrm{CDCl}_{3}\right) \delta 6.83$ (br s, 1H), 6.36 (br s, 1H), $6.32(\mathrm{~s}, 1 \mathrm{H}), 5.94(\mathrm{~s}, 2 \mathrm{H}), 5.32(\mathrm{~d}, J=10.6 \mathrm{~Hz}$, $1 \mathrm{H}), 5.29(\mathrm{~d}, J=6.1 \mathrm{~Hz}, 1 \mathrm{H}), 5.23(\mathrm{~d}, J=6.0 \mathrm{~Hz}, 1 \mathrm{H}), 4.64(\mathrm{~s}, 1 \mathrm{H}), 3.83(\mathrm{~s}, 3 \mathrm{H}), 3.79(\mathrm{~s}, 3 \mathrm{H}), 3.55(\mathrm{~s}, 3 \mathrm{H}), 1.62-1.53(\mathrm{~m}, 1 \mathrm{H})$, $1.48(\mathrm{~s}, 3 \mathrm{H}), 1.04-0.95(\mathrm{~m}, 30 \mathrm{H}), 0.70(\mathrm{~d}, J=6.9 \mathrm{~Hz}, 3 \mathrm{H}), 0.22(\mathrm{~s}, 3 \mathrm{H}), 0.13(\mathrm{~s}, 3 \mathrm{H}), 0.11(\mathrm{~s}, 3 \mathrm{H}) ;{ }^{13} \mathrm{C} \mathrm{NMR}(126 \mathrm{MHz} \mathrm{CDCl})$ $\delta 153.2,149.1,148.2,140.0,138.1,136.4,136.1,135.2,114.7,110.5,106.5,103.0,102.0,97.2,79.6,73.6,60.6,57.5$, 
55.9, 42.7, 25.8, 20.7, 18.5, 18.3, 18.2, 12.9, 12.1, $-0.9,-4.58,-4.63$; $[\alpha]^{23} \mathrm{D}+7.2^{\circ},\left(\mathrm{c}=1.1, \mathrm{CHCl}_{3}\right)$; HRMS: Calcd. for $\mathrm{C}_{39} \mathrm{H}_{64} \mathrm{BBrO}_{10} \mathrm{Si}_{2}[\mathrm{M}+\mathrm{Na}]^{+}=861.3207 \mathrm{~m} / \mathrm{z}$, found $=861.3212 \mathrm{~m} / \mathrm{z}$.

\section{Compound SI-4}

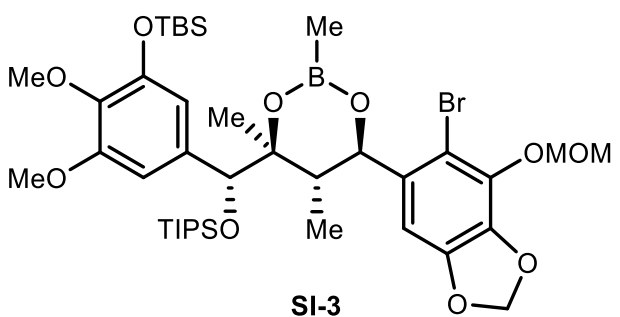

Chemical Formula: $\mathrm{C}_{39} \mathrm{H}_{64} \mathrm{BBrO}_{10} \mathrm{Si}_{2}$ Molecular Weight: 839.8150

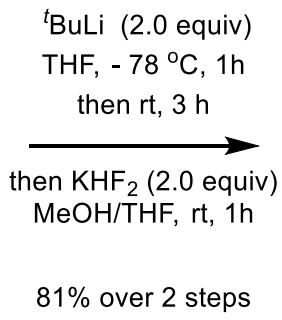

$81 \%$ over 2 steps<smiles>COc1cc([C@H](OC(C)(F)F)[C@](C)(O)[C@H](C)[C@H]2OB(C)c3c2cc2c(c3OC)OCO2)cc(OC)c1OC</smiles>

Procedure: A $500 \mathrm{~mL}$ flame-dried round bottom flask equipped with a Teflon-coated stir bar and a rubber septum was charged with SI-3 (14.59 mmol, 12.25 g, 1.0 equiv). The flask was evacuated and refill with $\mathrm{N}_{2}$ (repeat 3 times), prior to the addition of THF $(150 \mathrm{~mL})$. The mixture was then cooled to $-78^{\circ} \mathrm{C}$ in a dry ice / acetone bath, at which point ${ }^{t} \mathrm{BuLi}(29.18$ mmol, 1.7 M pentane, $17.2 \mathrm{~mL}, 2.0$ equiv) was added dropwise over $30 \mathrm{~min}$ with a syringe pump. The resulting mixture was stirred at $-78{ }^{\circ} \mathrm{C}$ for $1 \mathrm{~h}$, and another $3 \mathrm{~h}$ at rt. It was then quenched by the addition of a solution consisting $20 \mathrm{~mL}$ of saturated $\mathrm{NH}_{4} \mathrm{Cl}$ and $\mathrm{H}_{2} \mathrm{O}(100 \mathrm{~mL})$, and extracted with EtOAc $(3 \times 100 \mathrm{~mL})$. The combined organic layers were dried over $\mathrm{MgSO}_{4}$, filtered and concentrated in vacuo. The crude product was dissolved in $\mathrm{MeOH}$ and THF $(1: 1,150 \mathrm{~mL})$, and KHF ( $29.18 \mathrm{mmol}, 2.28 \mathrm{~g}, 2.0$ equiv) was added as solid. The resulting solution was stirred at $\mathrm{rt}$ for $1 \mathrm{~h}$, dilute with PhMe (50 $\mathrm{mL}$ ), and concentrated in vacuo to $\sim 50 \mathrm{~mL}$. $\mathrm{H}_{2} \mathrm{O}(50 \mathrm{~mL})$ and brine $(50 \mathrm{~mL})$ was then added, the resulting mixture was extracted with EtOAc $(3 \times 100 \mathrm{~mL})$. The combined organic layers were dried over $\mathrm{MgSO}_{4}$, filtered and concentrated in vacuo. The crude product was purified on silica gel (5:1 to 3:1 hexanes/EtOAc) to provide SI-4 (7.63 g, 11.80 mmol, $81 \%)$ as a white foam.

\section{Characterization:}

$\mathbf{R}_{\boldsymbol{f}}=$ (hexanes/EtOAc 5:1): 0.14; IR (neat) $v=3511,2942,2866,1599,1508,1464,1433,1324,1248,1201,1159,1139 \mathrm{~cm}$ 1; ${ }^{1} \mathrm{H}$ NMR $\left(500 \mathrm{MHz}, \mathrm{CDCl}_{3}\right) \delta 6.72(\mathrm{~s}, 1 \mathrm{H}), 6.69$ (br s, 1H), $6.58(\mathrm{br} \mathrm{s}, 1 \mathrm{H}), 5.96(\mathrm{br} \mathrm{s}, 2 \mathrm{H}), 5.76(\mathrm{~s}, 1 \mathrm{H}), 5.46(\mathrm{~d}, J=3.4 \mathrm{~Hz}$, $1 \mathrm{H}), 5.37(\mathrm{~d}, J=6.0 \mathrm{~Hz}, 1 \mathrm{H}), 5.29(\mathrm{~d}, J=6.0 \mathrm{~Hz}, 1 \mathrm{H}), 4.63(\mathrm{~s}, 1 \mathrm{H}), 3.90(\mathrm{~s}, 3 \mathrm{H}), 3.85(\mathrm{~s}, 3 \mathrm{H}), 3.53(\mathrm{~s}, 3 \mathrm{H}), 2.81(\mathrm{~s}, 1 \mathrm{H}), 2.37-$ $2.25(\mathrm{~m}, 1 \mathrm{H}), 1.28(\mathrm{~s}, 3 \mathrm{H}), 1.07-0.92(\mathrm{~m}, 21 \mathrm{H}), 0.79(\mathrm{~s}, 3 \mathrm{H}), 0.63(\mathrm{~d}, J=7.2 \mathrm{~Hz}, 3 \mathrm{H}) ;{ }^{13} \mathrm{C} \mathrm{NMR}(126 \mathrm{MHz}, \mathrm{CDCl}) \delta 153.1$, 152.7, 152.0, 148.5, 141.6, 137.1, 135.2, 135.1, 124.7, 108.6, 104.5, 101.4, 99.5, 96.6, 87.9, 81.9, 77.6, 61.2, 57.0, 56.0,

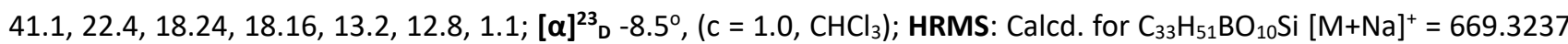
$\mathrm{m} / \mathrm{z}$, found $=669.3229 \mathrm{~m} / \mathrm{z}$.

\section{Compound 29}<smiles>COc1cc([C@H](O[Si])[C@@](C)(O)[C@H](C)[C@H]2OB(C)c3c2cc2c(c3OC)OCO2)cc(O)c1OC</smiles>

Chemical Formula: $\mathrm{C}_{33} \mathrm{H}_{51} \mathrm{BO}_{10} \mathrm{Si}$ Molecular Weight: 646.6560

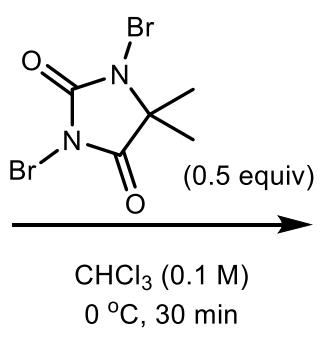

$97 \%$<smiles>COc1cc([C@H](O[Si])[C@@](C)(O)[C@H](C)[C@H]2OB(C)c3c2cc2c(c3OC)OCO2)c(Br)c(O)c1OC</smiles>

Chemical Formula: $\mathrm{C}_{33} \mathrm{H}_{50} \mathrm{BBrO}_{10} \mathrm{Si}$ Molecular Weight: 725.5520

Procedure: A $500 \mathrm{~mL}$ flame-dried round bottom flask equipped with a Teflon-coated stir bar and a rubber septum was 
charged with SI-4 (15.48 mmol, $10.01 \mathrm{~g}, 1.0$ equiv) and dissolved in $\mathrm{CHCl}_{3}(150 \mathrm{~mL})$. The mixture was then cooled to $0{ }^{\circ} \mathrm{C}$ in an ice bath, at which point 1,3-Dibromo-5,5-dimethylhydantoin (DBDMH, $7.74 \mathrm{~mol}, 2.21 \mathrm{~g}, 0.5$ equiv) was added as solid in portions (an orange color appeared when the first portion of DBDMH was added, the next portion was added after the orange color faint). The resulting solution was stirred at $0{ }^{\circ} \mathrm{C}$ for $30 \mathrm{~min}$ and concentrated in vacuo. The crude product was purified on silica gel (3:1 hexanes/EtOAc) to provide 29 (10.91 g, $15.04 \mathrm{mmol}, 97 \%)$ as an off-white solid. Single crystals for $\mathrm{X}$-ray diffraction were crystallized by vapor diffusion from Hexanes and EtOAc.

\section{Characterization:}

$\mathbf{R}_{\boldsymbol{f}}=$ (hexanes/EtOAc 3:1): 0.32; IR (neat) $v=3464,2942,2866,1604,1575,1464,1426,1334,1247,1220,1194,1153$, $1108 \mathrm{~cm}^{-1} ;{ }^{1} \mathrm{H} \mathrm{NMR}\left(500 \mathrm{MHz}, \mathrm{CDCl}_{3}\right) \delta 7.00(\mathrm{~s}, 1 \mathrm{H}), 6.65(\mathrm{~s}, 1 \mathrm{H}), 6.14(\mathrm{~s}, 1 \mathrm{H}), 5.95(\mathrm{~s}, 2 \mathrm{H}), 5.48(\mathrm{~d}, J=3.6 \mathrm{~Hz}, 1 \mathrm{H}), 5.38(\mathrm{~d}$, $J=6.0 \mathrm{~Hz}, 1 \mathrm{H}), 5.31(\mathrm{~s}, 1 \mathrm{H}), 5.28(\mathrm{~d}, J=6.0 \mathrm{~Hz}, 1 \mathrm{H}), 3.93(\mathrm{~s}, 3 \mathrm{H}), 3.84(\mathrm{~s}, 3 \mathrm{H}), 3.53(\mathrm{~s}, 3 \mathrm{H}), 2.73(\mathrm{~s}, 1 \mathrm{H}), 2.23(\mathrm{qd}, J=7.1,3.8$ $\mathrm{Hz}, 1 \mathrm{H}), 1.35(\mathrm{~s}, 3 \mathrm{H}), 1.09-1.03(\mathrm{~m}, 3 \mathrm{H}), 1.01(\mathrm{~d}, J=6.3 \mathrm{~Hz}, 9 \mathrm{H}), 0.91(\mathrm{~d}, J=6.9 \mathrm{~Hz}, 9 \mathrm{H}), 0.80(\mathrm{~d}, J=7.2 \mathrm{~Hz}, 3 \mathrm{H}), 0.77(\mathrm{~s}$, 3H); ${ }^{13} \mathrm{C}$ NMR (126 MHz, CDCl $)$ $\delta$ 153.0, 152.7, 151.1, 146.0, 141.5, 136.3, 135.7, 135.1, 124.7, 106.4, 103.4, 101.4, 99.6, $96.5,87.7,78.8,78.3,61.3,57.0,56.0,41.5,22.8,18.2,18.0,13.7,12.7,1.0 ;[\alpha]^{23}{ }_{\mathrm{D}}+7.0^{\circ},\left(\mathrm{c}=0.8, \mathrm{CHCl}_{3}\right) ; \mathrm{HRMS}$ : Calcd. for $\mathrm{C}_{33} \mathrm{H}_{50} \mathrm{BBrO}_{10} \mathrm{Si}[\mathrm{M}+\mathrm{Na}]^{+}=747.2342 \mathrm{~m} / \mathrm{z}$, found $=747.2355 \mathrm{~m} / \mathrm{z}$.

\section{Compound 30}

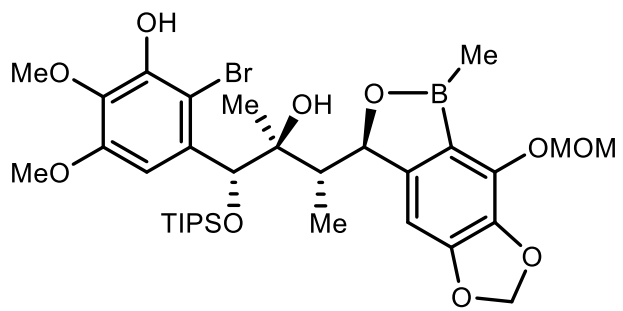

29

Chemical Formula: $\mathrm{C}_{33} \mathrm{H}_{50} \mathrm{BBrO}_{10} \mathrm{Si}$ Molecular Weight: 725.5520

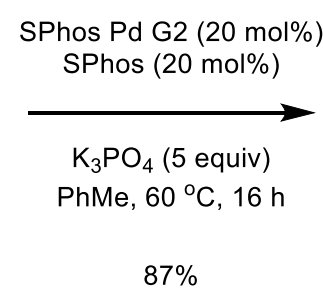

$87 \%$

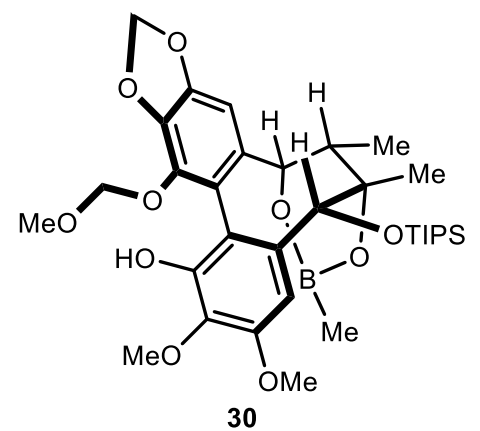

Chemical Formula: $\mathrm{C}_{33} \mathrm{H}_{49} \mathrm{BO}{ }_{10} \mathrm{Si}$ Molecular Weight: 644.6400

Procedure: A $500 \mathrm{~mL}$ flame-dried Schlenk flask equipped with a Teflon-coated stir bar and a rubber septum was charged with 29 (10.67 mmol, $7.74 \mathrm{~g}, 1.0$ equiv), SPhos Pd G2 (2.13 mmol, $1.53 \mathrm{~g}, 20 \mathrm{~mol} \%$ ), SPhos (2.13 mol, $874.4 \mathrm{mg}, 20 \mathrm{~mol} \%$ ) and $\mathrm{K}_{3} \mathrm{PO}_{4}\left(53.35 \mathrm{mmol}, 11.32 \mathrm{~g}, 5.0\right.$ equiv). The flask was evacuated and refill with $\mathrm{N}_{2}$ (repeat 3 times), prior to the addition of PhMe $(213 \mathrm{~mL})$. The mixture was then warmed to $60^{\circ} \mathrm{C}$ in a pre-heated oil bath for $16 \mathrm{~h}$. It was then poured on a mixture consisting $\mathrm{H}_{3} \mathrm{PO}_{4}(2 \mathrm{M}, 50 \mathrm{~mL})$ and brine $(50 \mathrm{~mL})$, and extracted with EtOAc $(3 \times 100 \mathrm{~mL})$. The combined organic layers were dried over $\mathrm{MgSO}_{4}$, filtered and concentrated in vacuo. The crude product was purified on silica gel (3:1 hexanes/EtOAc) to provide $\mathbf{3 0}(6.00 \mathrm{~g}, 9.31 \mathrm{mmol}, 87 \%)$ as a pale-yellow foam.

\section{Characterization:}

$\mathbf{R}_{f}=$ (hexanes/EtOAc 3:1): 0.22; IR (neat) $v=3398,2938,2865,1614,1460,1368,1334,1303,1272,1248,1231,1192$, 1140, $1106 \mathrm{~cm}^{-1} ;{ }^{1} \mathrm{H}$ NMR $\left(500 \mathrm{MHz}, \mathrm{CDCl}_{3}\right) \delta 6.78(\mathrm{~s}, 1 \mathrm{H}), 6.38(\mathrm{~s}, 1 \mathrm{H}), 5.96(\mathrm{~d}, J=1.3 \mathrm{~Hz}, 1 \mathrm{H}), 5.95(\mathrm{~d}, J=1.3 \mathrm{~Hz}, 1 \mathrm{H}), 5.74$ $(\mathrm{s}, 1 \mathrm{H}), 5.20(\mathrm{~d}, J=5.2 \mathrm{~Hz}, 1 \mathrm{H}), 5.14(\mathrm{~d}, J=5.2 \mathrm{~Hz}, 1 \mathrm{H}), 4.81(\mathrm{~s}, 1 \mathrm{H}), 4.78(\mathrm{~s}, 1 \mathrm{H}), 3.91(\mathrm{~s}, 3 \mathrm{H}), 3.89(\mathrm{~s}, 3 \mathrm{H}), 3.38(\mathrm{~s}, 3 \mathrm{H}), 1.96$ (q, J = 7.2 Hz, $1 \mathrm{H}), 1.40(\mathrm{~s}, 3 \mathrm{H}), 1.22(\mathrm{~d}, J=7.2 \mathrm{~Hz}, 3 \mathrm{H}), 0.89(\mathrm{~s}, 21 \mathrm{H}),-0.38(\mathrm{~s}, 3 \mathrm{H}) ;{ }^{13} \mathrm{C}$ NMR $\left(126 \mathrm{MHz}, \mathrm{CDCl}_{3}\right) \delta 151.2$, 148.9, 146.0, 138.3, 136.7, 135.5, 134.3, 117.6, 114.8, 104.6, 102.1, 101.3, 97.0, 81.7, 78.0, 61.1, 57.0, 55.8, 43.7, 23.4, $18.24,18.17,12.6,-1.8 ;[\alpha]^{23}{ }_{D}-76.8^{\circ},\left(c=1.1, \mathrm{CHCl}_{3}\right)$; HRMS: Calcd. for $\mathrm{C}_{33} \mathrm{H}_{49} \mathrm{BO}_{10} \mathrm{Si}[\mathrm{M}+\mathrm{Na}]^{+}=667.3080 \mathrm{~m} / \mathrm{z}$, found $=$ $667.3056 \mathrm{~m} / \mathrm{z}$. 


\section{Compound SI-5}

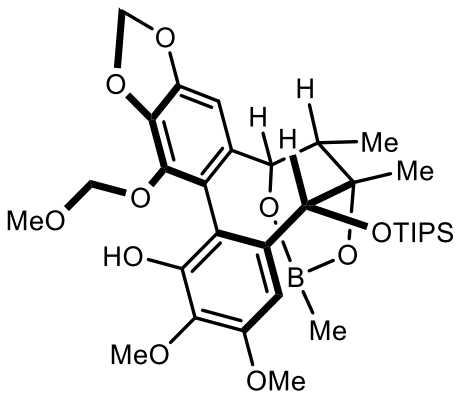

30

Chemical Formula: $\mathrm{C}_{33} \mathrm{H}_{49} \mathrm{BO}_{10} \mathrm{Si}$ Molecular Weight: 644.6400

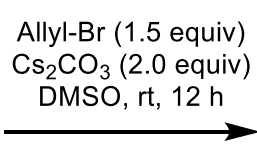

then $\mathrm{NaOH}, \mathrm{H}_{2} \mathrm{O}_{2}$ $\mathrm{MeOH}, 0^{\circ} \mathrm{C}, 2 \mathrm{~h}$

$95 \%$

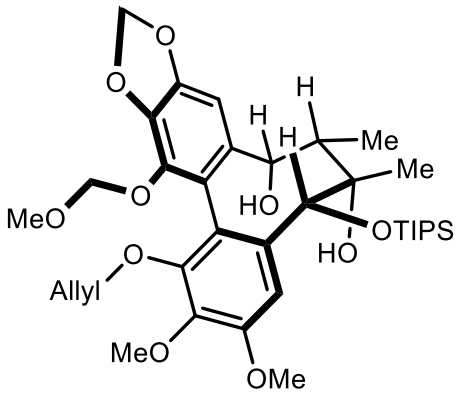

SI-5

Chemical Formula: $\mathrm{C}_{35} \mathrm{H}_{52} \mathrm{O}_{10} \mathrm{Si}$ Molecular Weight: 660.8760

Procedure: A $250 \mathrm{~mL}$ flame-dried round bottom flask equipped with a Teflon-coated stir bar and a rubber septum was charged with 30 (9.31 mmol, $6.00 \mathrm{~g}, 1.0$ equiv) and $\mathrm{Cs}_{2} \mathrm{CO}_{3}$ (18.62 mmol, $6.07 \mathrm{~g}, 2.0$ equiv). The flask was evacuated and refill with $\mathrm{N}_{2}$ (repeat 3 times), prior to the addition of DMSO $(20 \mathrm{~mL})$ and allyl bromide $(13.97 \mathrm{mmol}, 1.21 \mathrm{~mL}, 1.5 \mathrm{equiv})$. The resulting mixture was stirred at $\mathrm{rt}$ for $12 \mathrm{~h}$. MeOH $(100 \mathrm{~mL})$ was then added, and the mixture was cooled to $0{ }^{\circ} \mathrm{C}$ $\mathrm{NaOH}\left(1 \mathrm{M}\right.$ in $\mathrm{H}_{2} \mathrm{O}, 30 \mathrm{~mL}$ ) and $30 \% \mathrm{H}_{2} \mathrm{O}_{2}$ (13.97 mmol, $1.43 \mathrm{~mL}, 1.5$ equiv) was then added, and the resulting mixture was stirred for $2 \mathrm{~h}$, quenched by the addition of $\mathrm{NaHCO}_{3}$ (saturated, $20 \mathrm{~mL}$ ) and $\mathrm{Na}_{2} \mathrm{~S}_{2} \mathrm{O}_{3}$ (saturated, $20 \mathrm{~mL}$ ), and concentrated in vacuo. It was then extracted with EtOAc $(3 \times 100 \mathrm{~mL})$. The combined organic layers were washed with $\mathrm{H}_{2} \mathrm{O}(100 \mathrm{~mL})$, brine $(100 \mathrm{~mL})$ and dried over $\mathrm{MgSO}_{4}$. It was then filtered and concentrated in vacuo. The crude product was purified on silica gel (3:1 hexanes/EtOAc) to provide SI-5 (5.88 g, $8.89 \mathrm{mmol}, 95 \%)$ as a pale-yellow foam.

\section{Characterization:}

$\mathbf{R}_{f}=$ (hexanes/EtOAc 3:1): 0.31; IR (neat) $v=3321,2942,2893,2866,1618,1600,1496,1460,1428,1373,1329,1289$, 1250, 1229, 1193, 1161, 1146, 1131, $1104 \mathrm{~cm}^{-1} ;{ }^{1} \mathrm{H}_{\mathrm{NMR}}\left(500 \mathrm{MHz}, \mathrm{CDCl}_{3}\right) \delta 7.09(\mathrm{~s}, 1 \mathrm{H}), 6.30(\mathrm{~s}, 1 \mathrm{H}), 5.99(\mathrm{~d}, J=1.3 \mathrm{~Hz}$, $1 \mathrm{H}$ ), 5.92 (d, $J=1.3 \mathrm{~Hz}, 1 \mathrm{H}$ ), 5.70 (dddd, $J=16.8,10.4,6.2,5.4 \mathrm{~Hz}, 1 \mathrm{H}$ ), 5.28 (d, $J=5.3 \mathrm{~Hz}, 1 \mathrm{H}), 5.15(\mathrm{~d}, J=5.3 \mathrm{~Hz}, 1 \mathrm{H}), 5.07$ (ddd, $J=17.3,3.2,1.6 \mathrm{~Hz}, 1 \mathrm{H}), 5.01$ (dd, $J=10.4,1.5 \mathrm{~Hz}, 1 \mathrm{H}), 4.74(\mathrm{~d}, J=5.0 \mathrm{~Hz}, 1 \mathrm{H}), 4.64(\mathrm{~s}, 1 \mathrm{H}), 4.42(\mathrm{ddt}, J=12.7,5.2$, $1.3 \mathrm{~Hz}, 1 \mathrm{H}), 4.29(\mathrm{ddt}, J=12.7,6.3,1.2 \mathrm{~Hz}, 1 \mathrm{H}), 3.89(\mathrm{~s}, 3 \mathrm{H}), 3.87(\mathrm{~s}, 3 \mathrm{H}), 3.46(\mathrm{~s}, 3 \mathrm{H}), 2.07(\mathrm{~s}, 1 \mathrm{H}), 1.75(\mathrm{q}, J=7.0 \mathrm{~Hz}, 1 \mathrm{H})$, $1.61(\mathrm{~d}, J=5.6 \mathrm{~Hz}, 1 \mathrm{H}), 1.38(\mathrm{~s}, 3 \mathrm{H}), 1.32(\mathrm{~d}, J=7.1 \mathrm{~Hz}, 3 \mathrm{H}), 0.89-0.82(\mathrm{~m}, 21 \mathrm{H}) ;{ }^{13} \mathrm{C} \mathrm{NMR}\left(126 \mathrm{MHz}, \mathrm{CDCl}_{3}\right) \delta 152.4$ 148.85, 148.83, 141.2, 138.2, 138.1, 136.3, 135.5, 135.1, 121.8, 118.9, 117.1, 109.3, 102.0, 101.2, 97.0, 83.4, 78.2, 76.0,

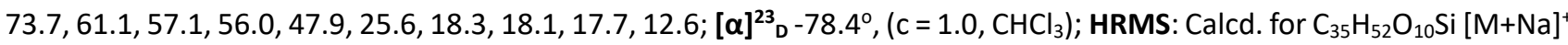
$=683.3222 \mathrm{~m} / \mathrm{z}$, found $=683.3218 \mathrm{~m} / \mathrm{z}$.

\section{Compound SI-6}

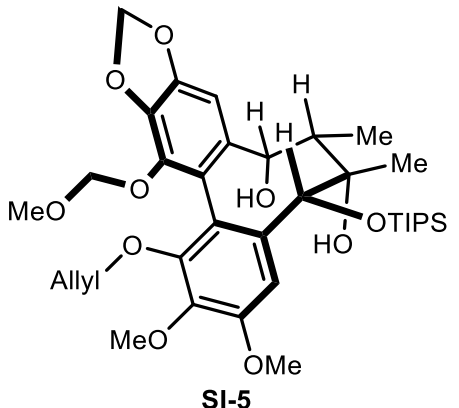

Chemical Formula: $\mathrm{C}_{35} \mathrm{H}_{52} \mathrm{O}_{10} \mathrm{Si}$ Molecular Weight: 660.8760

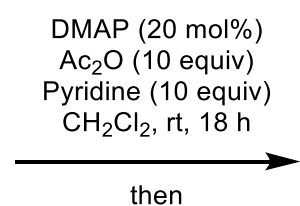

TBAF $3 \mathrm{H}_{2} \mathrm{O}$ (3 equiv) HOAc, THF, rt, $24 \mathrm{~h}$

$73 \%$ over 2 steps

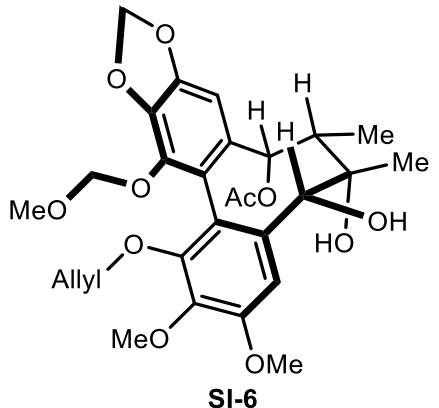

Chemical Formula: $\mathrm{C}_{28} \mathrm{H}_{34} \mathrm{O}_{11}$ Molecular Weight: 546.5690

Procedure: A $250 \mathrm{~mL}$ flame-dried round bottom flask equipped with a Teflon-coated stir bar and a rubber septum was charged with SI-5 (9.0 mmol, $5.93 \mathrm{~g}, 1.0$ equiv) and 4-( $N, N$-dimethylamino)pyridine (DMAP, $1.8 \mathrm{mmol}, 219.9 \mathrm{mg}, 20 \mathrm{~mol} \%$ ). 
The flask was evacuated and refill with $\mathrm{N}_{2}$ (repeat 3 times), prior to the addition of $\mathrm{CH}_{2} \mathrm{Cl}_{2}$ ( $45 \mathrm{~mL}$ ). Pyridine (90 mmol, $7.25 \mathrm{~mL}, 10$ equiv) and $\mathrm{Ac}_{2} \mathrm{O}(90 \mathrm{mmol}, 8.51 \mathrm{~mL}, 10$ equiv) was then added via syringe, and the resulting mixture was stirred at $\mathrm{rt}$ for $18 \mathrm{~h}$. It was then cooled to $0^{\circ} \mathrm{C}$ in an ice bath, and quenched by the addition of $\mathrm{MeOH}(90 \mathrm{mmol}, 3.64 \mathrm{~mL}$, 10 equiv) dropwise. The mixture was warmed to rt and stirred for another $30 \mathrm{~min}$, poured on $\mathrm{HCl}(1 \mathrm{M}, 150 \mathrm{~mL})$, and extracted with $\mathrm{CH}_{2} \mathrm{Cl}_{2}(3 \times 100 \mathrm{~mL})$. The combined organic fractions were washed with $\mathrm{NaHCO}_{3}$ (saturated, $100 \mathrm{~mL}$ ) and brine $(100 \mathrm{~mL})$, dried over $\mathrm{MgSO}_{4}$, filtered and concentrated in vacuo. The crude product was then evacuated and refill with $\mathrm{N}_{2}$ (repeat 3 times), prior to the addition of THF ( $18 \mathrm{~mL}$ ). A solution of TBAF.3 $\mathrm{H}_{2} \mathrm{O}(27 \mathrm{mmol}, 8.52 \mathrm{~g}, 3.0$ equiv) and HOAC (10.8 mmol, $617.7 \mu \mathrm{L}, 1.2$ equiv) in THF ( $27 \mathrm{~mL}$ ) was then added, and the resulting mixture was stirred at rt for 24 h. It was then poured on $\mathrm{NaHCO}_{3}$ (saturated, $50 \mathrm{~mL}$ ) and brine $(50 \mathrm{~mL})$, and extracted with EtOAc $(3 \times 100 \mathrm{~mL})$. The combined organic layers were dried over $\mathrm{MgSO}_{4}$, filtered and concentrated in vacuo. The crude product was purified on silica gel (1:2 hexanes/EtOAc) to provide SI-6 $(3.58 \mathrm{~g}, 6.55 \mathrm{mmol}, 73 \%)$ as a pale-yellow foam.

\section{Characterization:}

$\mathbf{R}_{f}=$ (hexanes/EtOAc 1:2): 0.28; IR (neat) $v=3568,3456,2937,1742,1623,1596,1497,1478,1459,1428,1414,1371$, 1325, 1225, 1192, 1148, $1107 \mathrm{~cm}^{-1} ;{ }^{1} \mathrm{H}$ NMR $\left(500 \mathrm{MHz}, \mathrm{CDCl}_{3}\right) \delta 7.07(\mathrm{~s}, 1 \mathrm{H}), 6.48(\mathrm{~s}, 1 \mathrm{H}), 6.00(\mathrm{~d}, J=1.3 \mathrm{~Hz}, 1 \mathrm{H}), 5.98(\mathrm{~d}$, $J=1.3 \mathrm{~Hz}, 1 \mathrm{H}$ ), $5.89-5.77(\mathrm{~m}, 1 \mathrm{H}), 5.62(\mathrm{~s}, 1 \mathrm{H}), 5.16(\mathrm{~d}, J=6.2 \mathrm{~Hz}, 1 \mathrm{H}), 5.10$ (ddd, $J=17.2,3.2,1.5 \mathrm{~Hz}, 1 \mathrm{H}$ ), 5.03 (dd, $J=$ 10.4, $1.5 \mathrm{~Hz}, 1 \mathrm{H}$ ), $4.82(\mathrm{~d}, J=6.2 \mathrm{~Hz}, 1 \mathrm{H}), 4.56(\mathrm{~s}, 1 \mathrm{H}), 4.45$ (ddt, $J=12.1,5.2,1.4 \mathrm{~Hz}, 1 \mathrm{H}$ ), 4.25 (ddt, J = 12.2, 5.5, $1.3 \mathrm{~Hz}$, $1 \mathrm{H}), 3.93(\mathrm{~s}, 3 \mathrm{H}), 3.87(\mathrm{~s}, 3 \mathrm{H}), 3.08(\mathrm{~s}, 3 \mathrm{H}), 1.99(\mathrm{br} \mathrm{s}, 1 \mathrm{H}), 1.91(\mathrm{q}, J=7.0 \mathrm{~Hz}, 1 \mathrm{H}), 1.62(\mathrm{~s}, 1 \mathrm{H}), 1.55(\mathrm{~s}, 3 \mathrm{H}), 1.36(\mathrm{~s}, 3 \mathrm{H})$, $1.19(\mathrm{~d}, J=7.1 \mathrm{~Hz}, 3 \mathrm{H}) ;{ }^{13} \mathrm{C}$ NMR $\left(126 \mathrm{MHz}, \mathrm{CDCl}_{3}\right) \delta 169.1,153.0,149.8,149.0,140.8,137.9,137.5,135.4,134.7,134.1$,

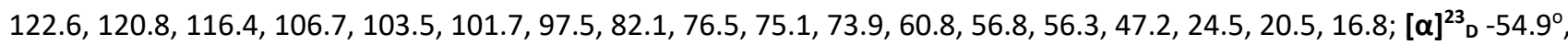
( $\mathrm{c}=1.1, \mathrm{CHCl}_{3}$ ); HRMS: Calcd. for $\mathrm{C}_{28} \mathrm{H}_{34} \mathrm{O}_{11}[\mathrm{M}+\mathrm{Na}]^{+}=569.1993 \mathrm{~m} / \mathrm{z}$, found $=569.1996 \mathrm{~m} / \mathrm{z}$.

\section{Compound 31}
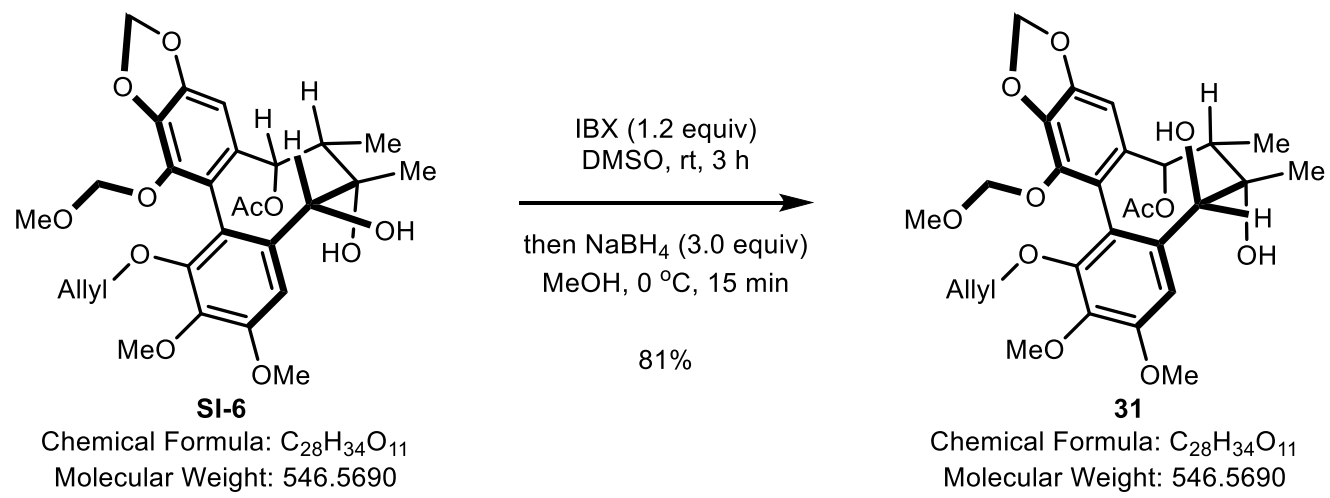

Procedure: A $250 \mathrm{~mL}$ flame-dried round bottom flask equipped with a Teflon-coated stir bar and a rubber septum was charged with SI-6 (6.40 mmol, $3.50 \mathrm{~g}, 1.0$ equiv) and iodoxybenzoic acid (IBX, $7.68 \mathrm{mmol}, 2.15 \mathrm{~g}, 1.2$ equiv). The flask was evacuated and refill with $\mathrm{N}_{2}$ (repeat 3 times), prior to the addition of DMSO $(32 \mathrm{~mL})$. The resulting mixture was stirred at rt for $3 \mathrm{~h}$. It was then cooled to $0{ }^{\circ} \mathrm{C}$ in an ice bath, at which point $\mathrm{MeOH}(32 \mathrm{~mL})$ was added. $\mathrm{NaBH}_{4}(19.2 \mathrm{mmol}, 726.3$ $\mathrm{mg}$, 3.0 equiv) was then added as solid in one portion. The mixture was stirred at $0{ }^{\circ} \mathrm{C}$ for another $15 \mathrm{~min}$, and quenched by the addition of $\mathrm{NH}_{4} \mathrm{Cl}$ (saturated, $\left.100 \mathrm{~mL}\right)$. It was then concentrated in vacuo, and extracted with EtOAc $(3 \times 100 \mathrm{~mL})$. The combined organic layers were washed with $\mathrm{NaHCO}_{3}$ (saturated, $\left.100 \mathrm{~mL}\right)$, water $(100 \mathrm{~mL})$ and brine $(50 \mathrm{~mL})$, dried over $\mathrm{MgSO}_{4}$, filtered and concentrated in vacuo. The crude product was purified on silica gel (1:1 hexanes/EtOAc) to provide 31 (2.84 g, $5.20 \mathrm{mmol}, 81 \%)$ as a white solid.

\section{Characterization:}

$\mathbf{R}_{f}=$ (hexanes/EtOAc 1:1): 0.32; IR (neat) $v=3568,3442,2979,2940,1743,1626,1598,1505,1480,1455,1432,1416$, 1369, 1331, 1253, 1226, 1196, 1146, $1111 \mathrm{~cm}^{-1} ;{ }^{1} \mathrm{H}$ NMR (500 MHz, CDCl $) \delta 6.69$ (s, 1H), 6.52 (s, 1H), 5.96 (br s, $\left.2 \mathrm{H}\right), 5.85$ 
$-5.71(\mathrm{~m}, 1 \mathrm{H}), 5.65(\mathrm{~s}, 1 \mathrm{H}), 5.09-5.03(\mathrm{~m}, 1 \mathrm{H}), 5.04(\mathrm{~d}, J=6.9 \mathrm{~Hz}, 1 \mathrm{H}), 5.00(\mathrm{dd}, J=10.4,1.4 \mathrm{~Hz}, 1 \mathrm{H}), 4.75(\mathrm{~s}, 1 \mathrm{H}), 4.74$ (d, $J=7.0 \mathrm{~Hz}, 1 \mathrm{H}), 4.41(\mathrm{dd}, J=12.1,5.3 \mathrm{~Hz}, 1 \mathrm{H}), 4.13(\mathrm{dd}, J=12.1,5.6 \mathrm{~Hz}, 1 \mathrm{H}), 3.88(\mathrm{~s}, 3 \mathrm{H}), 3.85(\mathrm{~s}, 3 \mathrm{H}), 3.08(\mathrm{~s}, 1 \mathrm{H}), 2.83$ (s, 3H), 2.09 (q, J = 7.2 Hz, 1H), $1.99(\mathrm{~s}, 1 \mathrm{H}), 1.54(\mathrm{~s}, 3 \mathrm{H}), 1.41(\mathrm{~s}, 3 \mathrm{H}), 1.23(\mathrm{~d}, J=7.2 \mathrm{~Hz}, 3 \mathrm{H}) ;{ }^{13} \mathrm{C} \mathrm{NMR}(126 \mathrm{MHz}, \mathrm{CDCl}) \delta$ 168.8, 152.0, 151.1, 148.8, 141.2, 138.4, 138.0, 134.4, 134.3, 132.9, 123.0, 122.3, 116.6, 110.4, 104.5, 101.9, 98.6, 85.9, $83.5,75.2,73.9,60.9,56.5,56.3,42.0,29.1,20.6,17.3 ;[\alpha]^{23}{ }_{\mathrm{D}}-102.5^{\circ}$, (c=1.0, $\left.\mathrm{CHCl}_{3}\right)$; HRMS: Calcd. for $\mathrm{C}_{28} \mathrm{H}_{34} \mathrm{O}_{11}[\mathrm{M}+\mathrm{Na}]^{+}$ $=569.1993 \mathrm{~m} / \mathrm{z}$, found $=569.1994 \mathrm{~m} / \mathrm{z}$.

\section{Compound SI-7}

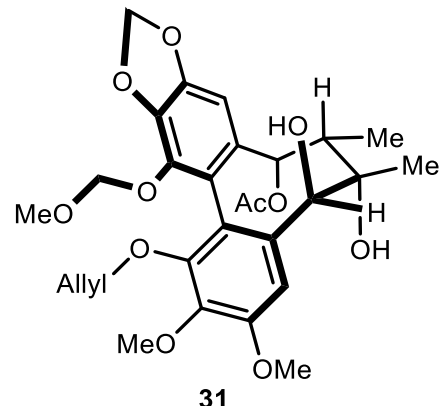

Chemical Formula: $\mathrm{C}_{28} \mathrm{H}_{34} \mathrm{O}_{11}$ Molecular Weight: 546.5690

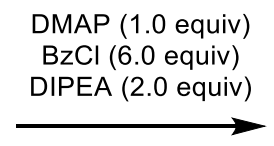

Pyridine, $60^{\circ} \mathrm{C}, 18 \mathrm{~h}$

$89 \%$

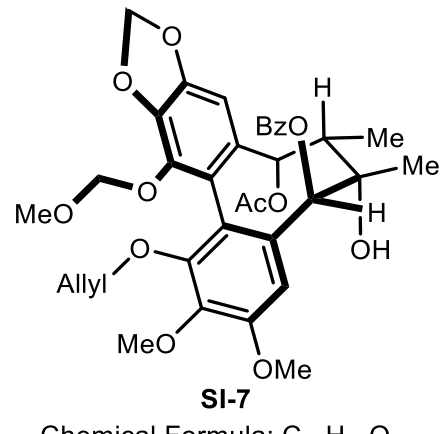

Chemical Formula: $\mathrm{C}_{35} \mathrm{H}_{38} \mathrm{O}_{12}$ Molecular Weight: 650.6770

Procedure: A $100 \mathrm{~mL}$ flame-dried round bottom flask equipped with a Teflon-coated stir bar and a rubber septum was charged with 31 (5.20 mmol, $2.84 \mathrm{~g}, 1.0$ equiv) and 4-( $N, N$-dimethylamino)pyridine (DMAP, $5.20 \mathrm{mmol}, 635.3 \mathrm{mg}, 1.0$ equiv). The flask was evacuated and refill with $\mathrm{N}_{2}$ (repeat 3 times), prior to the addition of pyridine (25 mL). DIPEA (10.4 $\mathrm{mmol}, 1.81 \mathrm{~mL}, 2.0$ equiv) and $\mathrm{BzCl}(31.2 \mathrm{mmol}, 3.62 \mathrm{~mL}, 6.0$ equiv) was then added via syringe, and the resulting mixture was stirred in a pre-heated oil bath at $60{ }^{\circ} \mathrm{C}$ for $18 \mathrm{~h}$. It was then cooled to rt and quenched by the addition of $\mathrm{MeOH}(2$ $\mathrm{mL}$ ) dropwise and stirred for another $30 \mathrm{~min}$ at $\mathrm{rt}$. The mixture was then diluted with EtOAc $(200 \mathrm{~mL})$, washed with $\mathrm{HCl}$ $(2 \mathrm{M}, 200 \mathrm{~mL})$, and the aqueous phase was then extracted with EtOAc $(2 \times 200 \mathrm{~mL})$. The combined organic layers were dried over $\mathrm{MgSO}_{4}$, filtered and concentrated in vacuo. The crude product was purified on silica gel (2:1 hexanes/EtOAc) to provide $\mathrm{SI-7}$ (3.02 g, $4.64 \mathrm{mmol}, 89 \%$ ) as an off-white solid.

\section{Characterization:}

$\mathbf{R}_{f}=$ (hexanes/EtOAc 2:1): 0.29; IR (neat) $v=3552,2985,2939,2909,1718,1626,1596,1500,1478,1451,1431,1371$, 1324, 1269, 1249, 1231, 1189, 1157, $1109 \mathrm{~cm}^{-1} ;{ }^{1} \mathrm{H}$ NMR $\left(500 \mathrm{MHz}, \mathrm{CDCl}_{3}\right) \delta 7.48(\mathrm{t}, J=7.4 \mathrm{~Hz}, 1 \mathrm{H}), 7.40$ (dd, J=8.3, 1.3 $\mathrm{Hz}, 2 \mathrm{H}), 7.29(\mathrm{t}, J=7.8 \mathrm{~Hz}, 2 \mathrm{H}), 6.86(\mathrm{~s}, 1 \mathrm{H}), 6.60(\mathrm{~s}, 1 \mathrm{H}), 6.02(\mathrm{~s}, 1 \mathrm{H}), 5.83(\mathrm{~d}, J=1.5 \mathrm{~Hz}, 1 \mathrm{H}), 5.80(\mathrm{~s}, 1 \mathrm{H}), 5.79-5.70(\mathrm{~m}$, $1 \mathrm{H}), 5.73(\mathrm{~d}, J=1.5 \mathrm{~Hz}, 1 \mathrm{H}), 5.03$ (ddd, $J=17.2,3.4,1.7 \mathrm{~Hz}, 1 \mathrm{H}), 4.98$ (ddd, $J=10.5,3.1,1.4 \mathrm{~Hz}, 1 \mathrm{H}$ ), $4.52(\mathrm{~d}, J=5.8 \mathrm{~Hz}$, $1 \mathrm{H}), 4.38$ (ddt, $J=12.3,5.1,1.5 \mathrm{~Hz}, 1 \mathrm{H}), 4.30$ (d, $J=5.8 \mathrm{~Hz}, 1 \mathrm{H}), 4.20$ (ddt, $J=12.3,5.4,1.5 \mathrm{~Hz}, 1 \mathrm{H}$ ), $3.94(\mathrm{~s}, 3 \mathrm{H}), 3.84(\mathrm{~s}$, $3 \mathrm{H}), 2.86(\mathrm{~s}, 3 \mathrm{H}), 2.29(\mathrm{q}, J=7.3 \mathrm{~Hz}, 1 \mathrm{H}), 2.14(\mathrm{~s}, 1 \mathrm{H}), 1.60(\mathrm{~s}, 3 \mathrm{H}), 1.36(\mathrm{~s}, 3 \mathrm{H}), 1.30(\mathrm{~d}, J=7.2 \mathrm{~Hz}, 3 \mathrm{H}) ;{ }^{13} \mathrm{C} \mathrm{NMR}(126 \mathrm{MHz}$, $\left.\mathrm{CDCl}_{3}\right) \delta 168.8,164.8,152.0,151.0,148.7,141.4,138.1,136.4,134.6,132.9,132.8,130.1,129.7,129.5,127.9,122.6$,

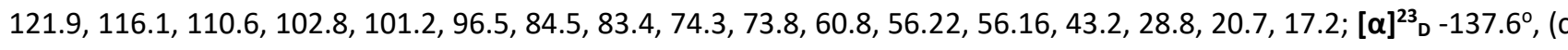
$\left.=1.0, \mathrm{CHCl}_{3}\right)$; HRMS: Calcd. for $\mathrm{C}_{35} \mathrm{H}_{38} \mathrm{O}_{12}[\mathrm{M}+\mathrm{Na}]^{+}=673.2256 \mathrm{~m} / \mathrm{z}$, found $=673.2256 \mathrm{~m} / \mathrm{z}$.

\section{Allyl bromoacetate (32)}<smiles>O=C(Br)CBr</smiles>

Chemical Formula: $\mathrm{C}_{2} \mathrm{H}_{2} \mathrm{Br}_{2} \mathrm{O}$ Molecular Weight: 201.8450

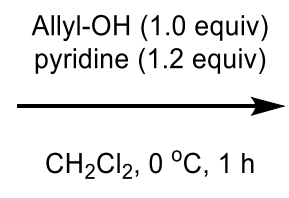

$90 \%$<smiles>O=C(CBr)O[Al]</smiles>

Chemical Formula: $\mathrm{C}_{5} \mathrm{H}_{7} \mathrm{BrO}_{2}$ Molecular Weight: 179.0130 
Procedure: A $500 \mathrm{~mL}$ flame-dried round bottom flask equipped with a Teflon-coated stir bar and a rubber septum was charged with allyl alcohol (100 mmol, $6.80 \mathrm{~mL}, 1.0$ equiv), pyridine ( $120 \mathrm{mmol}, 9.67 \mathrm{~mL}, 1.2$ equiv) and dissolved in $\mathrm{CH}_{2} \mathrm{Cl}_{2}$ (250 mL). It was cooled to $0{ }^{\circ} \mathrm{C}$ in an ice bath, at which point bromoacetyl bromide (100 mmol, $8.71 \mathrm{~mL}, 1.0$ equiv) was added dropwise via syringe. The resulting mixture was stirred at $0{ }^{\circ} \mathrm{C}$ for $1 \mathrm{~h}$, and quenched by the addition of $\mathrm{HCl}(1 \mathrm{M}$, $200 \mathrm{~mL})$. The mixture was then extracted with $\mathrm{CH}_{2} \mathrm{Cl}_{2}(3 \times 200 \mathrm{~mL})$. The combined organic layers were dried over $\mathrm{MgSO}_{4}$, filtered and concentrated in vacuo. The crude product was purified on silica gel (20:1 hexanes/EtOAc) to provide 32 (16.09 $\mathrm{g}, 89.9 \mathrm{mmol}, 90 \%)$ as a colorless oil.

\section{Characterization:}

${ }^{1} \mathrm{H}$ NMR $\left(500 \mathrm{MHz}, \mathrm{CDCl}_{3}\right) \delta 5.89$ (ddt, $J=17.1,10.5,5.8 \mathrm{~Hz}, 1 \mathrm{H}$ ), 5.34 (dq, $J=17.2,1.4 \mathrm{~Hz}, 1 \mathrm{H}$ ), 5.25 (dq, $J=10.4,1.2 \mathrm{~Hz}$, $1 \mathrm{H}), 4.63(\mathrm{dt}, J=5.8,1.3 \mathrm{~Hz}, 2 \mathrm{H}), 3.83(\mathrm{~s}, 2 \mathrm{H}) ;{ }^{13} \mathrm{C}$ NMR $\left(126 \mathrm{MHz}, \mathrm{CDCl}_{3}\right) \delta 166.9,131.3,119.1,66.7,25.8$. Our characterization data matches the data reported previously. ${ }^{8}$

\section{Compound SI-8}

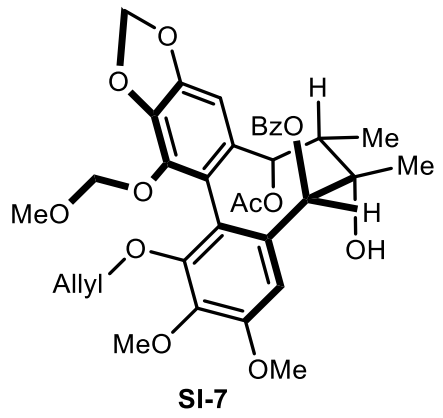

Chemical Formula: $\mathrm{C}_{35} \mathrm{H}_{38} \mathrm{O}_{12}$ Molecular Weight: 650.6770
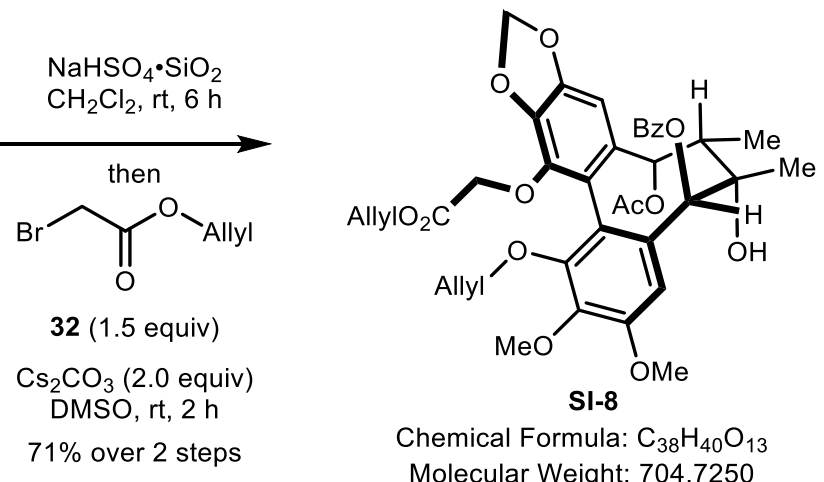

Chemical Formula: $\mathrm{C}_{38} \mathrm{H}_{40} \mathrm{O}_{13}$ Molecular Weight: 704.7250

Procedure: The literature procedure was followed for the first step with slight modifications. ${ }^{9} \mathrm{~A} 100 \mathrm{~mL}$ flame-dried round bottom flask equipped with a Teflon-coated stir bar and a rubber septum was charged with SI-7 $(3.60 \mathrm{mmol}, 2.34 \mathrm{~g}, 1.0$ equiv) and $\mathrm{NaHSO}_{4} \cdot \mathrm{SiO}_{2}\left(3.60 \mathrm{~g}\right.$ ). The flask was evacuated and refill with $\mathrm{N}_{2}$ (repeat 3 times), prior to the addition of $\mathrm{CH}_{2} \mathrm{Cl}_{2}$ $(72 \mathrm{~mL})$. The resulting mixture was stirred vigorously at $\mathrm{rt}$ for $6 \mathrm{~h}$, filtered through celite, and concentrated in vacuo. To the crude product was added $\mathrm{CS}_{2} \mathrm{CO}_{3}\left(7.20 \mathrm{mmol}, 2.35 \mathrm{~g}\right.$, 2.0 equiv). The flask was evacuated and refill with $\mathrm{N}_{2}$ (repeat 3 times), prior to the addition of DMSO (10 mL). Allyl bromoacetate (32) $(5.40 \mathrm{mmol}, 673.6 \mu \mathrm{L}, 1.5$ equiv) was then added via syringe, and the resulting mixture was stirred at $\mathrm{rt}$ for $2 \mathrm{~h}$. It was then diluted with $\mathrm{H}_{2} \mathrm{O}(50 \mathrm{~mL})$ and extracted with EtOAc $(3 \times 50 \mathrm{~mL})$. The combined organic layers were washed with $\mathrm{H}_{2} \mathrm{O}(100 \mathrm{~mL})$ and brine $(50 \mathrm{~mL})$, dried over $\mathrm{MgSO}_{4}$, filtered and concentrated in vacuo. The crude product was purified on silica gel (2:1 hexanes/EtOAc) to provide SI-8 (1.81 g, $2.57 \mathrm{mmol}, 71 \%)$ as an off-white solid. Single crystals for X-ray diffraction were crystallized by vapor diffusion from Hexanes and EtOAc.

\section{Characterization:}

$\mathbf{R}_{f}=$ (hexanes/EtOAc 2:1): 0.22; IR (neat) $v=3569,3428,2980,2939,1743,1626,1597,1504,1480,1454,1431,1416$, 1369, 1331, 1252, 1225, 1195, 1146, $1109 \mathrm{~cm}^{-1} ;{ }^{1}{ }^{H}$ NMR (500 MHz, CDCl $) \delta 7.50$ (t, $J=7.4 \mathrm{~Hz}, 1 \mathrm{H}$ ), 7.45 (dd, $J=8.3,1.2$ $\mathrm{Hz}, 2 \mathrm{H}), 7.31(\mathrm{t}, J=7.8 \mathrm{~Hz}, 2 \mathrm{H}), 6.83(\mathrm{~s}, 1 \mathrm{H}), 6.56(\mathrm{~s}, 1 \mathrm{H}), 5.90(\mathrm{~s}, 1 \mathrm{H}), 5.77(\mathrm{~s}, 1 \mathrm{H}), 5.85-5.69(\mathrm{~m}, 2 \mathrm{H}), 5.76(\mathrm{~d}, J=1.6 \mathrm{~Hz}$, $1 \mathrm{H}), 5.60(\mathrm{~d}, J=1.5 \mathrm{~Hz}, 1 \mathrm{H}), 5.20-5.11(\mathrm{~m}, 2 \mathrm{H}), 5.05$ (ddd, $J=17.2,3.4,1.7 \mathrm{~Hz}, 1 \mathrm{H}), 4.98$ (ddd, $J=10.5,3.1,1.4 \mathrm{~Hz}, 1 \mathrm{H}$ ), $4.52-4.43(\mathrm{~m}, 3 \mathrm{H}), 4.22(\mathrm{ddt}, J=12.3,5.5,1.5 \mathrm{~Hz}, 1 \mathrm{H}), 4.06(\mathrm{~d}, J=16.1 \mathrm{~Hz}, 1 \mathrm{H}), 3.95(\mathrm{~s}, 3 \mathrm{H}), 3.92(\mathrm{~d}, J=16.0 \mathrm{~Hz}, 1 \mathrm{H}), 3.83$ (s, 3H), $2.30(\mathrm{q}, J=7.1 \mathrm{~Hz}, 1 \mathrm{H}), 2.13(\mathrm{~s}, 1 \mathrm{H}), 1.59(\mathrm{~s}, 3 \mathrm{H}), 1.38(\mathrm{~s}, 3 \mathrm{H}), 1.31(\mathrm{~d}, J=7.2 \mathrm{~Hz}, 3 \mathrm{H}) ;{ }^{13} \mathrm{C} \mathrm{NMR}\left(126 \mathrm{MHz}, \mathrm{CDCl}_{3}\right) \delta$ $168.9,168.4,164.6,152.2,151.0,148.7,141.3,139.3,135.6,134.9,133.1,133.0,131.9,129.9,129.6,129.4,128.0,121.7$, $120.8,118.2,116.0,110.3,102.7,101.2,85.2,83.3,74.1,73.8,68.2,65.2,60.7,56.1,43.3,29.0,20.6,17.2 ;[\alpha]^{23}{ }_{\mathrm{D}}-125.8^{\circ}$, ( $\mathrm{c}=1.0, \mathrm{CHCl}_{3}$ ); HRMS: Calcd. for $\mathrm{C}_{38} \mathrm{H}_{40} \mathrm{O}_{13}[\mathrm{M}+\mathrm{Na}]^{+}=727.2361 \mathrm{~m} / \mathrm{z}$, found $=727.2365 \mathrm{~m} / \mathrm{z}$. 


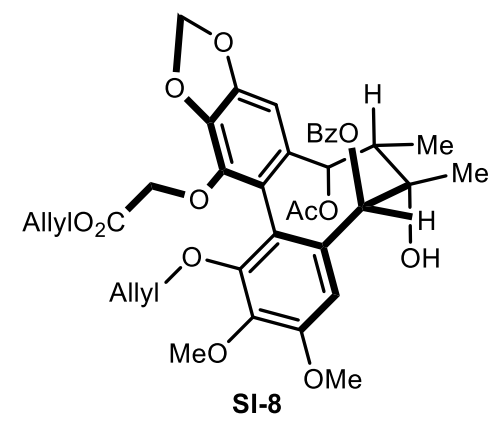

Chemical Formula: $\mathrm{C}_{38} \mathrm{H}_{40} \mathrm{O}_{13}$

Molecular Weight: 704.7250
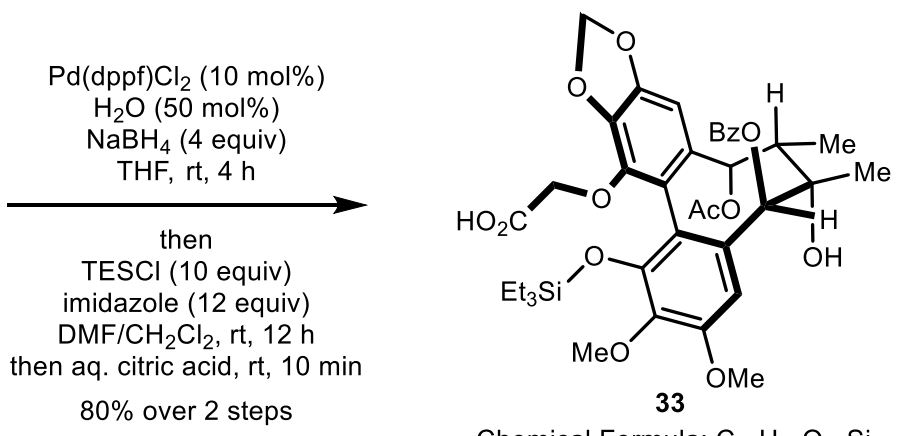

Chemical Formula: $\mathrm{C}_{38} \mathrm{H}_{46} \mathrm{O}_{13} \mathrm{Si}$ Molecular Weight: 738.8580

Procedure: A $100 \mathrm{~mL}$ flame-dried round bottom flask equipped with a Teflon-coated stir bar and a rubber septum was charged with SI-8 (1.80 mmol, $1.27 \mathrm{~g}, 1.0$ equiv), $\mathrm{Pd}(\mathrm{dppf}) \mathrm{Cl}_{2}(0.18 \mathrm{mmol}, 131.7 \mathrm{mg}, 10 \mathrm{~mol} \%)$ and $\mathrm{NaBH}_{4}(7.20 \mathrm{mmol}$, $272.4 \mathrm{mg}, 4.0$ equiv). The flask was evacuated and refill with $\mathrm{N}_{2}$ (repeat 3 times), prior to the addition of THF (18 mL) and $\mathrm{H}_{2} \mathrm{O}(0.9 \mathrm{mmol}, 16.2 \mu \mathrm{L}, 0.5$ equiv). The resulting mixture was stirred at $\mathrm{rt}$ for $4 \mathrm{~h}$. It was then poured on a mixture of $\mathrm{HCl}$ $(1 \mathrm{M}, 30 \mathrm{~mL})$ and brine $(30 \mathrm{~mL})$, and extracted with EtOAc $(3 \times 50 \mathrm{~mL})$. The combined organic layers were washed with $\mathrm{H}_{2} \mathrm{O}(100 \mathrm{~mL})$ and brine $(50 \mathrm{~mL})$, dried over $\mathrm{MgSO}_{4}$, filtered and concentrated in vacuo. To the crude product was added imidazole (21.6 mmol, $1.47 \mathrm{~g}, 12.0$ equiv), DMF ( $18 \mathrm{~mL}$ ), $\mathrm{CH}_{2} \mathrm{Cl}_{2}(18 \mathrm{~mL}$ ) and TESCl ( $18.0 \mathrm{mmol}, 3.02 \mathrm{~mL}, 10.0$ equiv). The resulting solution was stirred at $\mathrm{rt}$ for $12 \mathrm{~h}$. Citric acid $(0.5 \mathrm{M}$ aq., $9 \mathrm{~mL})$ was then added and stirred for another $10 \mathrm{~min}$. It was then diluted with EtOAc $(50 \mathrm{~mL})$ and acidified by the addition of $\mathrm{HCl}(1 \mathrm{M}, 50 \mathrm{~mL})$. The mixture was then extracted with EtOAc $(3 \times 50 \mathrm{~mL})$. The combined organic layers were washed with $\mathrm{H}_{2} \mathrm{O}(100 \mathrm{~mL})$ and brine $(50 \mathrm{~mL})$, dried over $\mathrm{MgSO}_{4}$, filtered and concentrated in vacuo. The crude product was purified on silica gel (2:1 hexanes/EtOAc to 10:1 EtOAc/MeOH) to provide 33 (1.06 g, $1.44 \mathrm{mmol}, 80 \%)$ as an off-white foam.

\section{Characterization:}

$\mathbf{R}_{f}=(E t O A c): 0.17 ; \mathbf{I R}$ (neat) $v=3569,2942,2876,1747,1721,1625,1596,1501,1479,1456,1428,1369,1337,1316$, 1250, 1220, 1193, 1148, $1109 \mathrm{~cm}^{-1} ;{ }^{1} \mathrm{H}$ NMR $\left(500 \mathrm{MHz}, \mathrm{CDCl}_{3}\right) \delta 7.53(\mathrm{t}, J=7.4 \mathrm{~Hz}, 1 \mathrm{H}), 7.44(\mathrm{~d}, J=7.2 \mathrm{~Hz}, 2 \mathrm{H}), 7.32(\mathrm{t}, J=$ $7.8 \mathrm{~Hz}, 2 \mathrm{H}), 6.76(\mathrm{~s}, 1 \mathrm{H}), 6.58(\mathrm{~s}, 1 \mathrm{H}), 5.83(\mathrm{~s}, 1 \mathrm{H}), 5.79(\mathrm{~s}, 1 \mathrm{H}), 5.74(\mathrm{~d}, J=1.2 \mathrm{~Hz}, 1 \mathrm{H}), 5.61(\mathrm{~d}, J=1.2 \mathrm{~Hz}, 1 \mathrm{H}), 4.28(\mathrm{~d}, J=$ $16.1 \mathrm{~Hz}, 1 \mathrm{H}), 3.95(\mathrm{~s}, 3 \mathrm{H}), 3.81(\mathrm{~s}, 3 \mathrm{H}), 3.80(\mathrm{~d}, J=16.1 \mathrm{~Hz}, 1 \mathrm{H}), 2.26(\mathrm{q}, J=7.3 \mathrm{~Hz}, 1 \mathrm{H}), 2.23(\mathrm{br} \mathrm{s}, 1 \mathrm{H}), 1.61(\mathrm{~s}, 3 \mathrm{H}), 1.39$ (s, 3H), 1.30 (d, $J=7.2 \mathrm{~Hz}, 3 \mathrm{H}$ ), $0.71(\mathrm{t}, J=7.9 \mathrm{~Hz}, 9 \mathrm{H}), 0.54$ (dq, $J=15.6,7.8 \mathrm{~Hz}, 3 \mathrm{H}), 0.43(\mathrm{dq}, J=16.1,8.0 \mathrm{~Hz}, 3 \mathrm{H}) ;{ }^{13} \mathrm{C}$ NMR $\left(126 \mathrm{MHz}, \mathrm{CDCl}_{3}\right) \delta 169.1,168.6,164.7,152.0,148.8,146.1,138.7,137.5,134.8,133.4,130.3,129.3,129.2,128.2$, $120.6,119.5,108.6,103.2,101.5,85.7,82.9,74.0,67.6,60.5,56.1,43.6,29.0,20.8,17.2,6.6,5.4 ;[\alpha]^{23} \mathrm{D}-80.1^{\circ},(c=1.1$, $\mathrm{CHCl}_{3}$ ); HRMS: Calcd. for $\mathrm{C}_{38} \mathrm{H}_{46} \mathrm{O}_{13} \mathrm{Si}[\mathrm{M}+\mathrm{Na}]^{+}=761.2600 \mathrm{~m} / \mathrm{z}$, found $=761.2601 \mathrm{~m} / \mathrm{z}$.

\section{Compound SI-9}

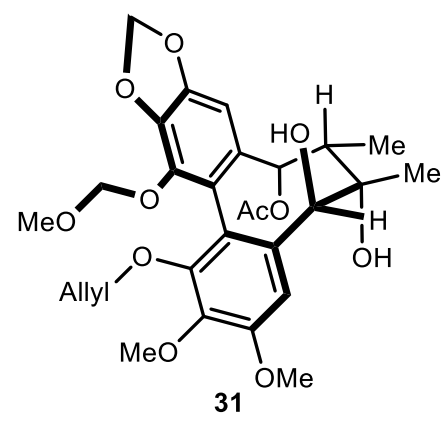

Chemical Formula: $\mathrm{C}_{28} \mathrm{H}_{34} \mathrm{O}_{11}$ Molecular Weight: 546.5690

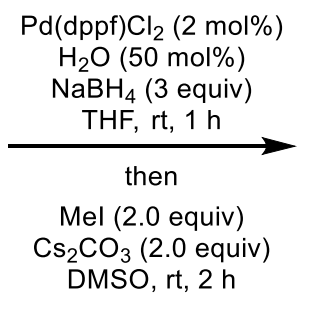

$96 \%$ over 2 steps

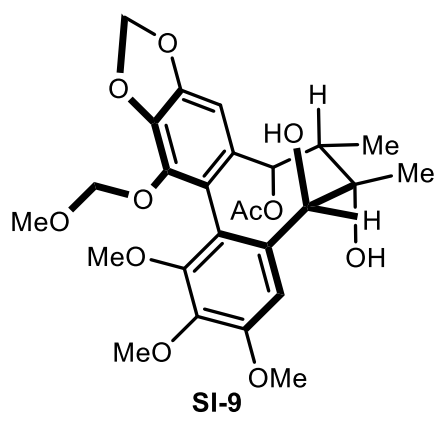

Chemical Formula: $\mathrm{C}_{26} \mathrm{H}_{32} \mathrm{O}_{11}$ Molecular Weight: 520.5310 
Procedure: A $50 \mathrm{~mL}$ flame-dried round bottom flask equipped with a Teflon-coated stir bar and a rubber septum was charged with 31 (0.80 mmol, $437.3 \mathrm{mg}, 1.0$ equiv) , $\mathrm{Pd}(\mathrm{dppf}) \mathrm{Cl}_{2}(0.016 \mathrm{mmol}, 11.7 \mathrm{mg}, 2 \mathrm{~mol} \%)$ and $\mathrm{NaBH}_{4}(2.40 \mathrm{mmol}$, $90.8 \mathrm{mg}, 3.0$ equiv). The vial was evacuated and refill with $\mathrm{N}_{2}$ (repeat 3 times), prior to the addition of $\mathrm{THF}(8 \mathrm{~mL}) \mathrm{and} \mathrm{H}_{2} \mathrm{O}$ $\left(0.40 \mathrm{mmol}, 7.2 \mu \mathrm{L}, 0.5\right.$ equiv). The resulting mixture was stirred at $\mathrm{rt}$ for $1 \mathrm{~h}$. It was then poured on saturated $\mathrm{NH}_{4} \mathrm{Cl}(50$ $\mathrm{mL}$ ) extracted with EtOAc $(3 \times 50 \mathrm{~mL})$. The combined organic layers were dried over $\mathrm{MgSO}_{4}$, filtered and concentrated in vacuo. To the crude product was added $\mathrm{Cs}_{2} \mathrm{CO}_{3}(1.60 \mathrm{mmol}, 521.3 \mathrm{mg}, 2.0$ equiv). The flask was evacuated and refill with $\mathrm{N}_{2}$ (repeat 3 times), prior to the addition of DMSO $(4 \mathrm{~mL})$. lodomethane (1.60 mmol, $99.6 \mu \mathrm{L}, 2.0$ equiv) was then added via syringe, and the resulting mixture was stirred at $\mathrm{rt}$ for $2 \mathrm{~h}$. It was then diluted with $\mathrm{H}_{2} \mathrm{O}(50 \mathrm{~mL})$ and extracted with EtOAc $(3 \times 50 \mathrm{~mL})$. The combined organic layers were washed with $\mathrm{H}_{2} \mathrm{O}(100 \mathrm{~mL})$ and brine $(50 \mathrm{~mL})$, dried over $\mathrm{MgSO}_{4}$, filtered and concentrated in vacuo. The crude product was purified on silica gel (1:1 hexanes/EtOAc) to provide SI-9 (400.0 $\mathrm{mg}, 0.768 \mathrm{mmol}, 96 \%)$ as a white foam.

\section{Characterization:}

$\mathbf{R}_{f}=$ (hexanes/EtOAc 3:1): 0.34; IR (neat) $v=3575,3468,2940,2843,1744,1628,1595,1500,1480,1460,1431,1410$, 1371, 1331, 1252, 1222, 1195, 1148, $1107 \mathrm{~cm}^{-1} ;{ }^{1} \mathbf{H}$ NMR $\left(500 \mathrm{MHz}, \mathrm{CDCl}_{3}\right) \delta 6.71(\mathrm{~s}, 1 \mathrm{H}), 6.54(\mathrm{~s}, 1 \mathrm{H}), 6.01(\mathrm{~d}, J=1.4 \mathrm{~Hz}$, $1 \mathrm{H}), 6.00(\mathrm{~d}, J=1.4 \mathrm{~Hz}, 1 \mathrm{H}), 5.63(\mathrm{~s}, 1 \mathrm{H}), 5.07(\mathrm{~d}, J=6.9 \mathrm{~Hz}, 1 \mathrm{H}), 4.77(\mathrm{~d}, J=1.4 \mathrm{~Hz}, 1 \mathrm{H}), 4.75(\mathrm{~d}, J=6.9 \mathrm{~Hz}, 1 \mathrm{H}), 3.90(\mathrm{~s}$, $3 \mathrm{H}), 3.88(\mathrm{~s}, 3 \mathrm{H}), 3.65(\mathrm{~s}, 3 \mathrm{H}), 3.05(\mathrm{~d}, J=1.9 \mathrm{~Hz}, 1 \mathrm{H}), 2.83(\mathrm{~s}, 3 \mathrm{H}), 2.11(\mathrm{q}, J=7.2 \mathrm{~Hz}, 1 \mathrm{H}), 2.03(\mathrm{~s}, 1 \mathrm{H}), 1.56(\mathrm{~s}, 3 \mathrm{H}), 1.43$ (s, 3H), 1.25 (d, $J=7.2 \mathrm{~Hz}, 3 \mathrm{H}) ;{ }^{13} \mathrm{C}$ NMR $\left(126 \mathrm{MHz} \mathrm{CDCl}_{3}\right) \delta 169.0,152.1,151.7,149.0,141.2,138.3,138.1,134.4,133.1$, $122.9,122.2,110.5,104.5,102.0,98.8,86.0,83.8,75.2,60.9,60.6,56.6,56.4,42.0,29.1,20.6,17.3 ;[\alpha]^{23}{ }_{D}-115.6^{\circ},(c=$ 1.0, $\mathrm{CHCl}_{3}$ ); HRMS: Calcd. for $\mathrm{C}_{26} \mathrm{H}_{32} \mathrm{O}_{11}[\mathrm{M}+\mathrm{Na}]^{+}=543.1837 \mathrm{~m} / \mathrm{z}$, found $=543.1820 \mathrm{~m} / \mathrm{z}$.

\section{Compound SI-10}
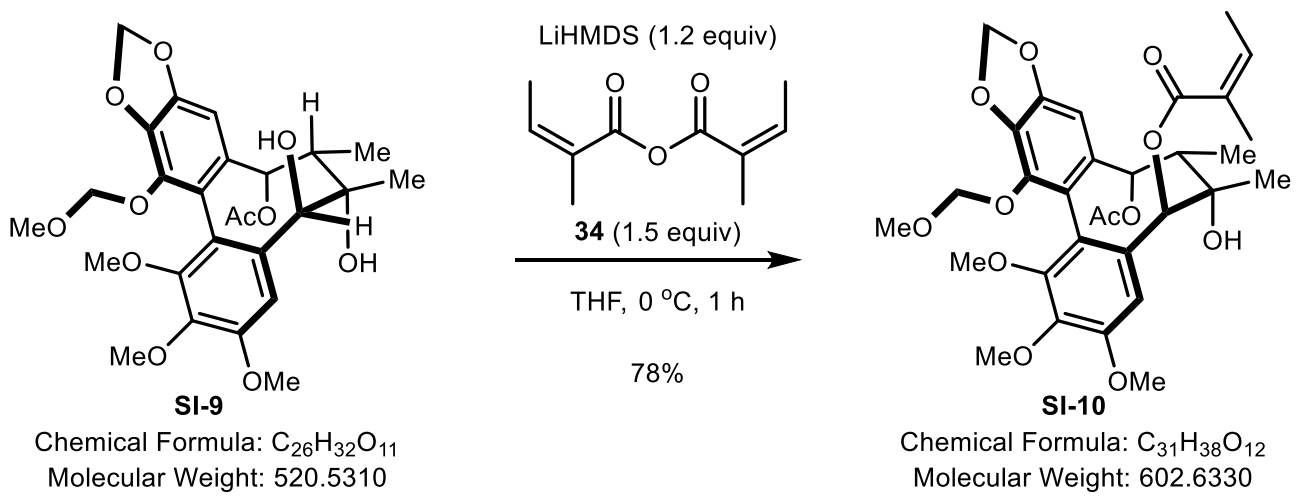

Procedure: The literature procedure was followed with slight modifications. ${ }^{10}$ A $50 \mathrm{~mL}$ flame-dried round bottom flask equipped with a Teflon-coated stir bar and a rubber septum was charged with SI-9 (0.71 mmol, $370.3 \mathrm{mg}, 1.0$ equiv). The flask was evacuated and refill with $\mathrm{N}_{2}$ (repeat 3 times), prior to the addition of THF $(10 \mathrm{~mL})$. It was cooled to $0{ }^{\circ} \mathrm{C}$ in an ice bath, at which point angelic anhydride (34) (1.07 mmol, $191.0 \mu \mathrm{L}, 1.5$ equiv) was then added, followed by dropwise addition a solution of LiHMDS ( $0.85 \mathrm{mmol}, 1 \mathrm{M}$ in THF, $853.0 \mu \mathrm{L}, 1.2$ equiv) by syringe. The resulting solution was then stirred for $1 \mathrm{~h}$ at $0{ }^{\circ} \mathrm{C}$ and quenched by the addition of $\mathrm{NH}_{4} \mathrm{Cl}$ (saturated, $10 \mathrm{~mL}$ ). The mixture was then extracted with EtOAc $(3 \times 50 \mathrm{~mL})$. The combined organic layers were dried over $\mathrm{MgSO}_{4}$, filtered and concentrated in vacuo. The crude product was purified on silica gel (2:1 hexanes/EtOAc) to provide SI-10 (335.8.0 mg, $0.557 \mathrm{mmol}, 78 \%)$ as a white solid.

\section{Characterization:}

$\mathbf{R}_{\boldsymbol{f}}=$ (hexanes/EtOAc 2:1): 0.29; IR (neat) $v=3530,2973,2941,1731,1712,1647,1626,1595,1501,1478,1459,1432$, 1413, 1369, 1335, 1269, 1250, 1224, 1145, $1106 \mathrm{~cm}^{-1} ;{ }^{1} \mathbf{H}$ NMR $\left(500 \mathrm{MHz}, \mathrm{CDCl}_{3}\right) \delta 6.81(\mathrm{~s}, 1 \mathrm{H}), 6.49(\mathrm{~s}, 1 \mathrm{H}), 5.98(\mathrm{qq}, J=$ 7.2, $1.4 \mathrm{~Hz}, 1 \mathrm{H}), 5.94(\mathrm{~d}, J=1.4 \mathrm{~Hz}, 1 \mathrm{H}), 5.92(\mathrm{~d}, J=1.4 \mathrm{~Hz}, 1 \mathrm{H}), 5.84(\mathrm{~s}, 1 \mathrm{H}), 5.71(\mathrm{~s}, 1 \mathrm{H}), 4.88(\mathrm{~d}, J=5.7 \mathrm{~Hz}, 1 \mathrm{H}), 4.84(\mathrm{~d}, J$ $=5.7 \mathrm{~Hz}, 1 \mathrm{H}), 3.93(\mathrm{~s}, 3 \mathrm{H}), 3.86(\mathrm{~s}, 3 \mathrm{H}), 3.63(\mathrm{~s}, 3 \mathrm{H}), 3.06(\mathrm{~s}, 3 \mathrm{H}), 2.13(\mathrm{q}, J=7.3 \mathrm{~Hz}, 1 \mathrm{H}), 2.12(\mathrm{~s}, 1 \mathrm{H}), 1.86(\mathrm{dq}, J=7.3,1.5$ 
$\mathrm{Hz}, 3 \mathrm{H}$ ), 1.59 (s, 3H), 1.33 (quint, $J=1.5 \mathrm{~Hz}, 3 \mathrm{H}), 1.31(\mathrm{~s}, 3 \mathrm{H}), 1.26(\mathrm{~d}, J=7.2 \mathrm{~Hz}, 3 \mathrm{H}) ;{ }^{13} \mathrm{C} \mathrm{NMR}(126 \mathrm{MHz}, \mathrm{CDCl}) \delta 168.9$, $165.9,152.0,151.5,148.7,141.3,140.3,138.0,136.9,132.8,130.3,127.1,122.3,121.9,110.7,103.3,101.4,97.2,84.1$,

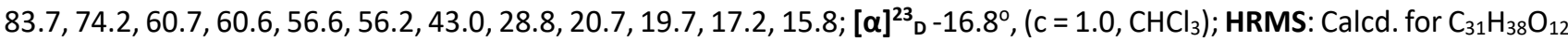
$[\mathrm{M}+\mathrm{K}]^{+}=641.1995 \mathrm{~m} / \mathrm{z}$, found $=641.1985 \mathrm{~m} / \mathrm{z}$.

\section{2-(Trimethylsilyl)ethyl bromoacetate (35)}<smiles>O=C(Br)CBr</smiles>

Chemical Formula: $\mathrm{C}_{2} \mathrm{H}_{2} \mathrm{Br}_{2} \mathrm{O}$ Molecular Weight: 201.8450

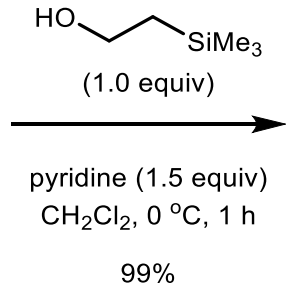

35

Chemical Formula: $\mathrm{C}_{7} \mathrm{H}_{15} \mathrm{BrO}_{2} \mathrm{Si}$ Molecular Weight: 239.1840

Procedure: A $100 \mathrm{~mL}$ flame-dried round bottom flask equipped with a Teflon-coated stir bar and a rubber septum was charged with 2-(trimethylsilyl)ethanol ( $20 \mathrm{mmol}, 2.87 \mathrm{~mL}, 1.0$ equiv), pyridine ( $30 \mathrm{mmol}, 2.42 \mathrm{~mL}, 1.5$ equiv) and dissolved in $\mathrm{CH}_{2} \mathrm{Cl}_{2}(40 \mathrm{~mL})$. It was cooled to $0^{\circ} \mathrm{C}$ in an ice bath, at which point bromoacetyl bromide $(20 \mathrm{mmol}, 1.74 \mathrm{~mL}, 1.0 \mathrm{equiv})$ was added dropwise via syringe. The resulting mixture was stirred at $0{ }^{\circ} \mathrm{C}$ for $1 \mathrm{~h}$, and quenched by the addition of $\mathrm{HCl}$ $(1 \mathrm{M}, 50 \mathrm{~mL})$. The mixture was then extracted with $\mathrm{CH}_{2} \mathrm{Cl}_{2}(3 \times 50 \mathrm{~mL})$. The combined organic layers were dried over $\mathrm{MgSO}_{4}$, filtered and concentrated in vacuo. The crude product was purified on silica gel (20:1 hexanes/EtOAc) to provide 35 (4.72 g, $19.7 \mathrm{mmol}, 99 \%)$ as a colorless oil.

\section{Characterization:}

${ }^{1} \mathrm{H}$ NMR $\left(500 \mathrm{MHz}, \mathrm{CDCl}_{3}\right) \delta 4.31-4.23(\mathrm{~m}, 2 \mathrm{H}), 3.81(\mathrm{~s}, 2 \mathrm{H}), 1.08-0.99(\mathrm{~m}, 2 \mathrm{H}), 0.06(\mathrm{~s}, 9 \mathrm{H}) ;{ }^{13} \mathrm{C} \mathrm{NMR}(126 \mathrm{MHz} \mathrm{CDCl})$ $\delta 167.5,64.9,26.2,17.4,-1.4$. Our characterization data matches the data reported previously. ${ }^{11}$

\section{Compound 36}

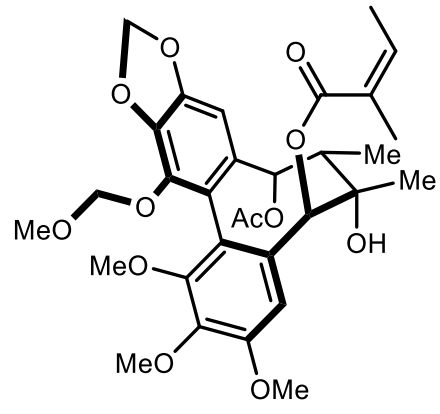

SI-10

Chemical Formula: $\mathrm{C}_{31} \mathrm{H}_{38} \mathrm{O}_{12}$ Molecular Weight: 602.6330

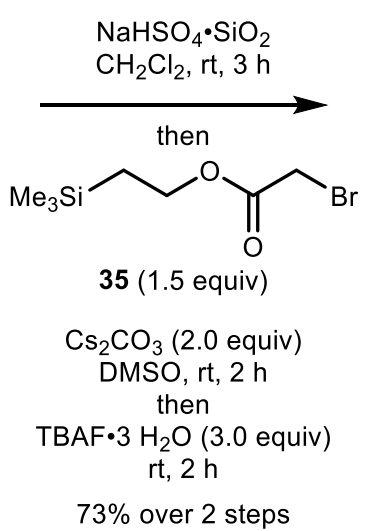

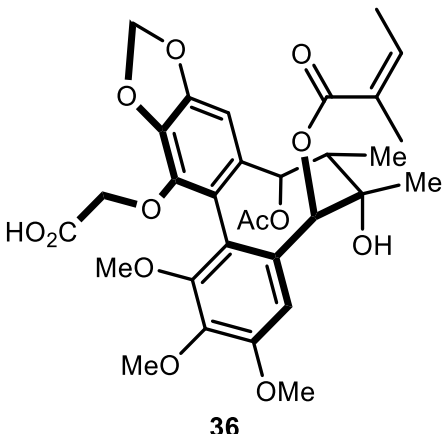

Chemical Formula: $\mathrm{C}_{31} \mathrm{H}_{36} \mathrm{O}_{13}$ Molecular Weight: 616.6160

Procedure: The literature procedure was followed for the first step with slight modifications. ${ }^{9} \mathrm{~A} 25 \mathrm{~mL}$ flame-dried round bottom flask equipped with a Teflon-coated stir bar and a rubber septum was charged with SI-10 (0.20 mmol, 120.5 mg, 1.0 equiv) and $\mathrm{NaHSO}_{4} \cdot \mathrm{SiO}_{2}\left(200 \mathrm{mg}\right.$ ). The flask was evacuated and refill with $\mathrm{N}_{2}$ (repeat 3 times), prior to the addition of $\mathrm{CH}_{2} \mathrm{Cl}_{2}(4 \mathrm{~mL})$. The resulting mixture was stirred vigorously at $\mathrm{rt}$ for $3 \mathrm{~h}$, filtered through celite, and concentrated in vacuo. To the crude product was added $\mathrm{Cs}_{2} \mathrm{CO}_{3}\left(0.40 \mathrm{mmol}, 130.3 \mathrm{mg}, 2.0\right.$ equiv). The flask was evacuated and refill with $\mathrm{N}_{2}$ (repeat 3 times), prior to the addition of DMSO (2 mL). 2-(Trimethylsilyl)ethyl bromoacetate (35) $(0.30 \mathrm{mmol}, 59.0 \mu \mathrm{L}, 1.5$ equiv) was then added via syringe, and the resulting mixture was stirred at $\mathrm{rt}$ for $2 \mathrm{~h}$. A solution of TBAF· $3 \mathrm{H} \mathrm{H}_{2} \mathrm{O}(0.60 \mathrm{mmol}$, $189.3 \mathrm{mg}, 3.0$ equiv) in THF ( $1 \mathrm{~mL}$ ) was added, and the resulting solution was stirred for another $2 \mathrm{~h}$. It was then diluted 
with $1 \mathrm{M} \mathrm{HCl}(20 \mathrm{~mL})$ and extracted with EtOAc $(3 \times 20 \mathrm{~mL})$. The combined organic layers were washed with $\mathrm{H}_{2} \mathrm{O}(50 \mathrm{~mL})$ and brine $(20 \mathrm{~mL})$, dried over $\mathrm{MgSO}_{4}$, filtered and concentrated in vacuo. The crude product was purified on silica gel (3:1 hexanes/EtOAc to 10:1 EtOAc/MeOH) to provide $36(89.8 \mathrm{mg}, 0.146 \mathrm{mmol}, 73 \%)$ as a white solid.

\section{Characterization:}

$\mathbf{R}_{f}=(\mathrm{EtOAC} / \mathrm{MeOH} 10: 1): 0.14 ; \mathbf{I R}$ (neat) $v=3590,2943,1744,1716,1624,1596,1502,1479,1459,1432,1412,1369$, 1335, 1223, 1197, 1147, $1104 \mathrm{~cm}^{-1} ;{ }^{1} \mathrm{H} \mathrm{NMR}\left(500 \mathrm{MHz}, \mathrm{CDCl}_{3}\right) \delta 10.50$ (br s, 1H), $6.90(\mathrm{~s}, 1 \mathrm{H}), 6.47(\mathrm{~s}, 1 \mathrm{H}), 6.02$ (qq, $J=7.1$, $1.2 \mathrm{~Hz}, 1 \mathrm{H}), 5.93(\mathrm{~d}, J=1.2 \mathrm{~Hz}, 1 \mathrm{H}), 5.91(\mathrm{~d}, J=1.1 \mathrm{~Hz}, 1 \mathrm{H}), 5.64(\mathrm{~s}, 1 \mathrm{H}), 5.62(\mathrm{~s}, 1 \mathrm{H}), 5.08(\mathrm{~d}, J=16.4 \mathrm{~Hz}, 1 \mathrm{H}), 4.44(\mathrm{~d}, J=$ $16.4 \mathrm{~Hz}, 1 \mathrm{H}), 3.95(\mathrm{~s}, 3 \mathrm{H}), 3.88(\mathrm{~s}, 3 \mathrm{H}), 3.55(\mathrm{~s}, 3 \mathrm{H}), 2.22(\mathrm{~s}, 1 \mathrm{H}), 2.12(\mathrm{q}, J=7.2 \mathrm{~Hz}, 1 \mathrm{H}), 1.84(\mathrm{dq}, J=7.2,1.4 \mathrm{~Hz}, 3 \mathrm{H}), 1.57$ (s, 3H), 1.39 (quint, $J=1.2 \mathrm{~Hz}, 3 \mathrm{H}$ ), $1.37(\mathrm{~s}, 3 \mathrm{H}), 1.28(\mathrm{~d}, J=7.2 \mathrm{~Hz}, 3 \mathrm{H}) ;{ }^{13} \mathrm{C}$ NMR $\left(126 \mathrm{MHz}, \mathrm{CDCl}_{3}\right) \delta 169.6,168.8,165.6$, $152.5,149.4,149.1,141.5,140.0,137.1,133.8,133.8,131.3,126.9,120.7,118.5,111.6,102.8,101.7,84.7,83.6,73.8$, $67.8,61.1,60.8,56.2,43.2,28.9,20.6,19.9,17.2,15.8 ;[\alpha]^{23}{ }_{\mathrm{D}}-24.8^{\circ}$, (c = 1.1, $\left.\mathrm{CHCl}_{3}\right)$; HRMS: Calcd. for $\mathrm{C}_{31} \mathrm{H}_{36} \mathrm{O}_{13}[\mathrm{M}+\mathrm{Na}]^{+}$ $=639.2048 \mathrm{~m} / \mathrm{z}$, found $=639.2039 \mathrm{~m} / \mathrm{z}$.

\section{Compound 3 (heteroclitin J)}

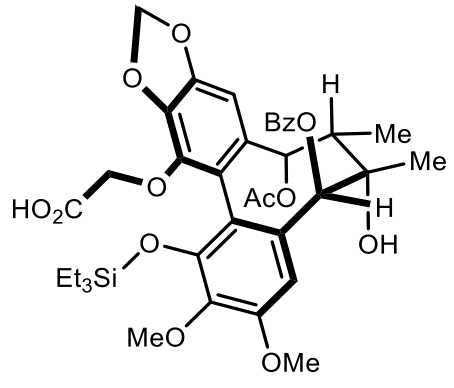

33

Chemical Formula: $\mathrm{C}_{38} \mathrm{H}_{46} \mathrm{O}_{13} \mathrm{Si}$ Molecular Weight: 738.8580

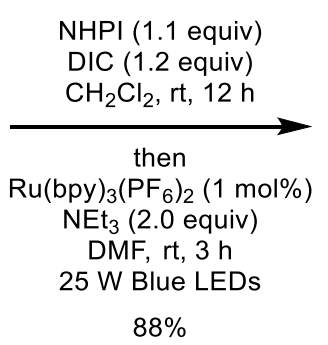

$88 \%$

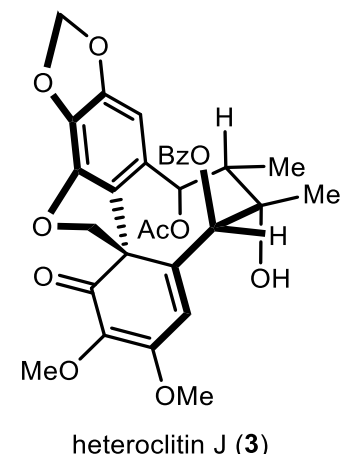

Chemical Formula: $\mathrm{C}_{31} \mathrm{H}_{30} \mathrm{O}_{11}$ Molecular Weight: 578.5700

Procedure: A $100 \mathrm{~mL}$ flame-dried round bottom flask equipped with a Teflon-coated stir bar and a rubber septum was charged with 33 ( $0.50 \mathrm{mmol}, 369.4 \mathrm{mg}, 1.0$ equiv) and $\mathrm{N}$-hydroxyphthalimide (NHPI, $0.55 \mathrm{mmol}, 89.7 \mathrm{mg}, 1.1$ equiv). The flask was evacuated and refill with $\mathrm{N}_{2}$ (repeat 3 times), prior to the addition of $\mathrm{CH}_{2} \mathrm{Cl}_{2}\left(5 \mathrm{~mL}\right.$ ), followed by $N, N^{\prime}$ diisopropylcarbodiimide (DIC, $0.60 \mathrm{mmol}, 94.0 \mu \mathrm{L}, 1.2$ equiv) via microsyringe. The resulting mixture was stirred for $12 \mathrm{~h}$ at $\mathrm{rt}$ under $\mathrm{N}_{2}$, and diluted by the addition of DMF (40 mL). A solution of Ru(bpy) ${ }_{3}\left(\mathrm{PF}_{6}\right)_{2}(0.005 \mathrm{mmol}, 4.30 \mathrm{mg}, 1.0 \mathrm{~mol} \%)$ in DMF ( $5 \mathrm{~mL}$ ) was added, followed by $\mathrm{NEt}_{3}(1.0 \mathrm{mmol}, 139.4 \mu \mathrm{L}, 2.0$ equiv). The resulting solution was then irradiated with $25 \mathrm{~W}$ blue LEDs at $\mathrm{rt}$ for $3 \mathrm{~h}$ with a cooling fan (See the picture below). It was then poured on a mixture of $\mathrm{H}_{2} \mathrm{O}$ (100 $\mathrm{mL})$ and $\mathrm{H}_{3} \mathrm{PO}_{4}(2 \mathrm{M}, 5 \mathrm{~mL})$, and extracted with EtOAc $(3 \times 100 \mathrm{~mL})$. The combined organic layers were washed with $\mathrm{H}_{2} \mathrm{O}$ $(2 \times 100 \mathrm{~mL})$ and brine $(50 \mathrm{~mL})$, dried over $\mathrm{MgSO}_{4}$, filtered and concentrated in vacuo. The crude product was purified on silica gel (4:1 to 1:1 hexanes/EtOAc) to provide $3(255.7 \mathrm{mg}, 0.44 \mathrm{mmol}, 88 \%)$ as a yellow solid. Single crystals for X-ray diffraction were crystallized by slow evaporation from $\mathrm{MeOH}$.

\section{Characterization:}

$\mathbf{R}_{\boldsymbol{f}}=$ (hexanes/EtOAc 1:1): 0.32; IR (neat) $\mathbf{v}=3570,3416,2937,1742,1716,1642,1627,1601,1583,1557,1504,1488$, $1451,1418,1398,1372,1315,1286,1262,1235,1176,1148,1126,1110 \mathrm{~cm}^{-1} ;{ }^{1} \mathrm{H} \mathrm{NMR}\left(500 \mathrm{MHz}, \mathrm{CDCl}_{3}\right) \delta 7.51(\mathrm{tt}, J=$ $7.1,1.6 \mathrm{~Hz}, 1 \mathrm{H}), 7.32(\mathrm{~d}, J=7.5 \mathrm{~Hz}, 2 \mathrm{H}), 7.29(\mathrm{t}, J=7.7 \mathrm{~Hz}, 2 \mathrm{H}), 6.51(\mathrm{~d}, J=1.2 \mathrm{~Hz}, 1 \mathrm{H}), 6.50(\mathrm{~s}, 1 \mathrm{H}), 5.93(\mathrm{~d}, J=1.4 \mathrm{~Hz}, 1 \mathrm{H})$, $5.89(\mathrm{~d}, J=1.1 \mathrm{~Hz}, 1 \mathrm{H}), 5.88(\mathrm{~d}, J=1.5 \mathrm{~Hz}, 1 \mathrm{H}), 5.88(\mathrm{~s}, 1 \mathrm{H}), 4.68(\mathrm{~d}, J=9.0 \mathrm{~Hz}, 1 \mathrm{H}), 4.06(\mathrm{~s}, 3 \mathrm{H}), 4.05(\mathrm{~d}, J=9.0 \mathrm{~Hz}, 1 \mathrm{H})$, $3.75(\mathrm{~s}, 3 \mathrm{H}), 2.54(\mathrm{~s}, 1 \mathrm{H}), 2.24(\mathrm{q}, J=7.2 \mathrm{~Hz}, 1 \mathrm{H}), 1.86(\mathrm{~s}, 3 \mathrm{H}), 1.31(\mathrm{~d}, J=7.2 \mathrm{~Hz}, 3 \mathrm{H}), 1.25(\mathrm{~s}, 3 \mathrm{H}) ;{ }^{13} \mathrm{C} \mathrm{NMR}(126 \mathrm{MHz}$, $\left.\mathrm{CDCl}_{3}\right) \delta 196.1,168.7,165.1,155.7,150.2,143.5,141.8,133.9,131.9,130.4,129.8,129.3,128.3,128.2,123.1,120.4$, 102.0, 101.2, 82.4, 81.5, 78.9, 75.2, 63.3, 59.0, 58.9, 44.2, 28.5, 20.4, 17.8; HPLC (Chiralpak ${ }^{\circledR}$ IC analytical column, eluent 
65:35 ${ }^{i} \mathrm{PrOH}$ :hexanes, $1 \mathrm{~mL} / \mathrm{min}$ ) $\mathrm{t}_{\mathrm{R}} 17.6 \mathrm{~min}$ (major), $21.2 \mathrm{~min}$ (minor), > 99\% e.e.; $[\alpha]^{23} \mathrm{D}-140.0^{\circ},(\mathrm{c}=0.50, \mathrm{MeOH})\left[\right.$ lit. ${ }^{1}$ $\left.[\alpha]^{20}{ }_{D}-60.5^{\circ},(c=0.16, \mathrm{MeOH})\right]$; HRMS: Calcd. for $\mathrm{C}_{31} \mathrm{H}_{30} \mathrm{O}_{11}[\mathrm{M}+\mathrm{Na}]^{+}=601.1680 \mathrm{~m} / \mathrm{z}$, found $=601.1677 \mathrm{~m} / \mathrm{z}$.

\section{Photoredox setup with blue LED light:}

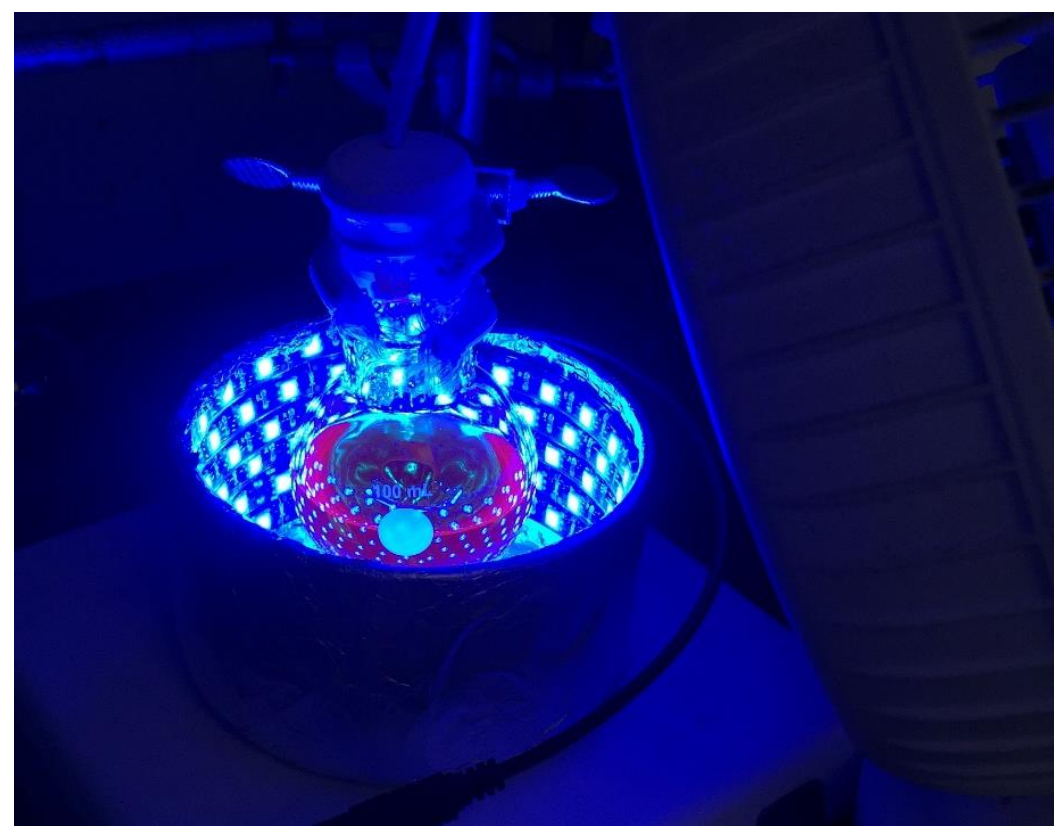

\section{HPLC trace of the racemic mixture:}

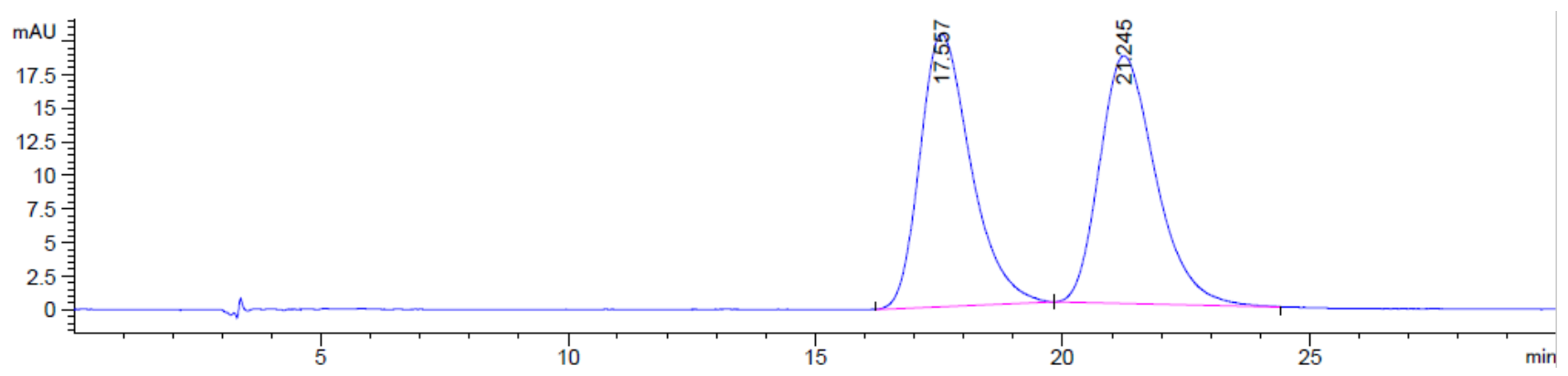

Signal 1: DAD1 A, Sig=328,2 Ref=off

\begin{tabular}{|c|c|c|c|c|c|c|}
\hline $\begin{array}{c}\text { Peak } \\
\#\end{array}$ & $\begin{array}{c}\text { RetTime } \\
\text { [min] }\end{array}$ & Type & $\begin{array}{l}\text { Width } \\
\text { [min] }\end{array}$ & $\begin{array}{c}\text { Area } \\
{\left[\mathrm{mAU}{ }^{*} \mathrm{~s}\right]}\end{array}$ & $\begin{array}{l}\text { Height } \\
\text { [mAU] }\end{array}$ & $\begin{array}{c}\text { Area } \\
\&\end{array}$ \\
\hline \multicolumn{7}{|c|}{$----|-------|----|-------|----------|----------|--------\mid$} \\
\hline 1 & 17.557 & $\mathrm{BB}$ & 1.0132 & 1449.48254 & 20.29629 & 49.9270 \\
\hline 2 & 21.245 & $B B$ & 1.0583 & 1453.71899 & 18.42894 & 50.0730 \\
\hline
\end{tabular}


HPLC trace of the chiral product:

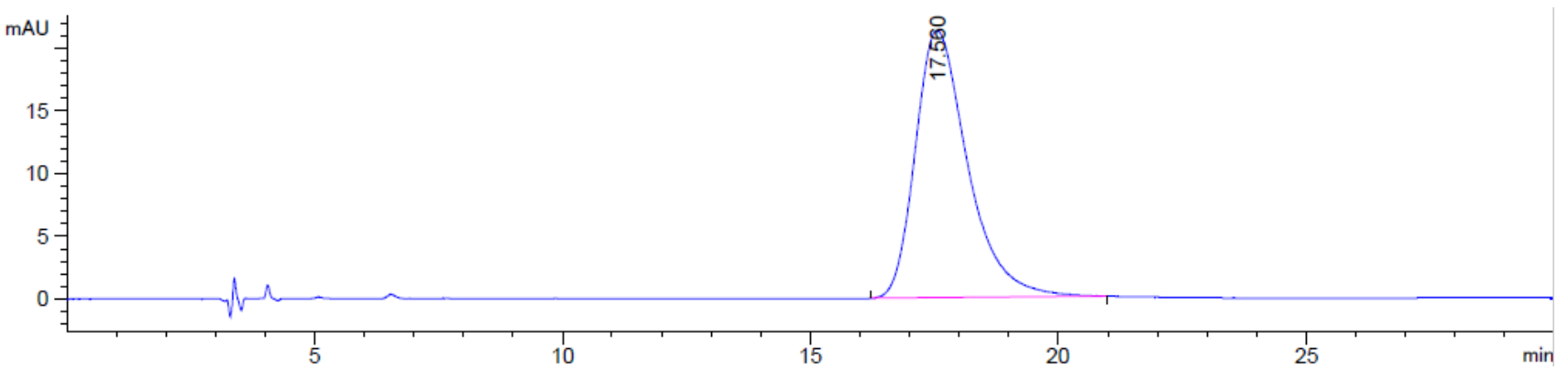

Signal 1: DAD1 A, Sig=328,2 Ref=off

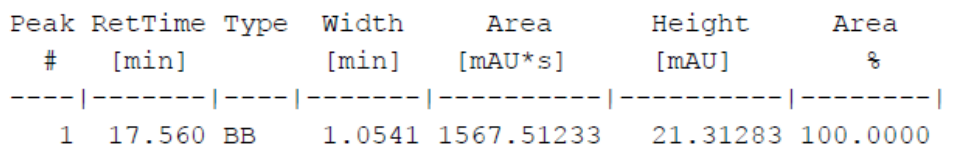

\section{Compounds 5a (taiwankadsurin A) and 5b (taiwankadsurin B)}

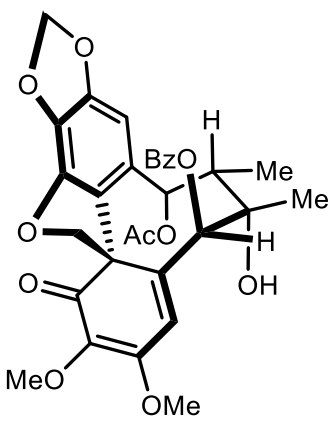

heteroclitin $\mathrm{J}(\mathbf{3})$

Chemical Formula: $\mathrm{C}_{31} \mathrm{H}_{30} \mathrm{O}_{11}$ Molecular Weight: 578.5700

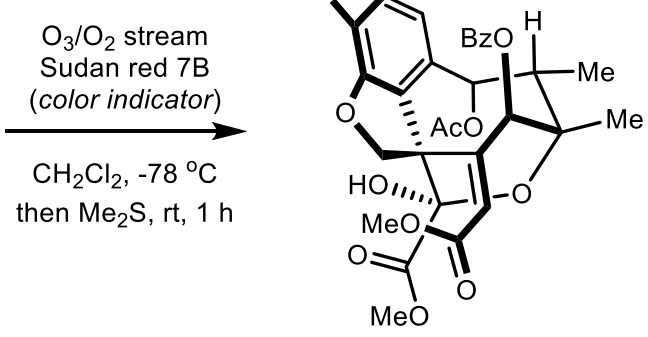

taiwankadsurin A (5a) $63 \%$

Chemical Formula: $\mathrm{C}_{31} \mathrm{H}_{30} \mathrm{O}_{13}$ Chemical Formula: $\mathrm{C}_{31} \mathrm{H}_{30} \mathrm{O}_{13}$ Molecular Weight: $610.5680 \quad$ Molecular Weight: 610.5680

Procedure: A $50 \mathrm{~mL}$ flame-dried round bottom flask equipped with a Teflon-coated stir bar was charged with 3 (0.10 mmol, $57.9 \mathrm{mg}, 1.0$ equiv) and dissolved in $\mathrm{CH}_{2} \mathrm{Cl}_{2}(20 \mathrm{~mL})$. A solution of Sudan red 7B (color indicator, $1 \mathrm{mg} / \mathrm{mL}$ in $\mathrm{CH}_{2} \mathrm{Cl}_{2}, 20 \mu \mathrm{L}$ ) was added, the resulting red solution was cooled to $-78{ }^{\circ} \mathrm{C}$, and bubbled with $\mathrm{N}_{2}$ for 5 min. The $\mathrm{N}_{2}$ stream was then removed, and a stream of $\mathrm{O}_{3} / \mathrm{O}_{2}$ was then introduced to the head space of the flask until the faint of the red color in the solution ( $5 \mathrm{~s}$ ). The $\mathrm{O}_{3} / \mathrm{O}_{2}$ stream was then quickly replaced by the $\mathrm{N}_{2}$ stream, and bubble for another $5 \mathrm{~min}$. $\mathrm{Me} \mathrm{S}_{2} \mathrm{~S}(2 \mathrm{~mL})$ was then added, and the resulting solution was capped by a rubber septum, warmed to rt and stirred for another $1 \mathrm{~h}$ under $\mathrm{N}_{2}$, and then concentrated in vacuo. The crude product was purified on silica gel (2:1 hexanes/EtOAc) to provide the major diastereomer (5a) $(38.2 \mathrm{mg}, 0.063 \mathrm{mmol}, 63 \%)$ as an off-white solid and the minor diastereomer (5b) (4.1 $\mathrm{mg}$, $0.007 \mathrm{mmol}, 7 \%)$ as an off-white solid.

\section{Characterization:}

Major diastereomer, taiwankadsurin A: $\mathbf{R}_{f}=$ (hexanes/EtOAc 2:1): 0.29; IR (neat) v = 3440, 2989, 2951, 1726, 1650, 1601, 1503, 1486, 1451, 1434, 1411, 1391, 1372, 1244, 1214, 1175, $1153 \mathrm{~cm}^{-1} ;{ }^{1} \mathbf{H}$ NMR $\left(500 \mathrm{MHz}, \mathrm{CDCl}_{3}\right) \delta 8.31$ (d, J = $7.2 \mathrm{~Hz}$, $2 \mathrm{H}), 7.65(\mathrm{t}, J=7.4 \mathrm{~Hz}, 1 \mathrm{H}), 7.53(\mathrm{t}, J=7.8 \mathrm{~Hz}, 2 \mathrm{H}), 6.69(\mathrm{~d}, J=3.0 \mathrm{~Hz}, 1 \mathrm{H}), 6.60(\mathrm{~s}, 1 \mathrm{H}), 6.49(\mathrm{~d}, J=2.7 \mathrm{~Hz}, 1 \mathrm{H}), 6.08(\mathrm{~d}, J=$ $2.7 \mathrm{~Hz}, 1 \mathrm{H}), 6.02(\mathrm{~d}, J=1.4 \mathrm{~Hz}, 1 \mathrm{H}), 5.99(\mathrm{~d}, J=1.4 \mathrm{~Hz}, 1 \mathrm{H}), 5.00(\mathrm{~d}, J=10.2 \mathrm{~Hz}, 1 \mathrm{H}), 4.54(\mathrm{~d}, J=10.2 \mathrm{~Hz}, 1 \mathrm{H}), 3.96(\mathrm{~s}, 3 \mathrm{H})$, $3.57(\mathrm{~s}, 3 \mathrm{H}), 3.51(\mathrm{~s}, 1 \mathrm{H}), 2.37(\mathrm{qd}, J=6.8,3.3 \mathrm{~Hz}, 1 \mathrm{H}), 2.21(\mathrm{~s}, 3 \mathrm{H}), 1.34(\mathrm{~s}, 3 \mathrm{H}), 1.05(\mathrm{~d}, J=6.8 \mathrm{~Hz}, 3 \mathrm{H}) ;{ }^{13} \mathrm{C} \mathrm{NMR}(126 \mathrm{MHz}$, $\left.\mathrm{CDCl}_{3}\right) \delta 171.1,169.2,165.7,165.5,150.5,150.4,144.3,134.2,130.7,129.5,129.0,128.5,127.9,118.2,117.4,102.0$, 
99.1, 97.7, 80.6, 79.4, 73.4, 70.6, 57.1, 53.8, 51.9, 45.3, 28.4, 21.4, 8.9; $[\alpha]^{23}{ }_{D}+35.0^{\circ},\left(\mathrm{c}=1.0, \mathrm{CH}_{2} \mathrm{Cl}_{2}\right)\left[\mathrm{lit}^{3}{ }^{3}[\alpha]^{26}{ }_{\mathrm{D}}+20^{\circ},(\mathrm{C}\right.$ $\left.\left.=0.5, \mathrm{CH}_{2} \mathrm{Cl}_{2}\right)\right]$; HRMS: Calcd. for $\mathrm{C}_{31} \mathrm{H}_{30} \mathrm{O}_{13}[\mathrm{M}+\mathrm{K}]^{+}=649.1318 \mathrm{~m} / \mathrm{z}$, found $=649.1287 \mathrm{~m} / \mathrm{z}$.

Minor diastereomer, taiwankadsurin B: $\mathbf{R}_{f}=$ (hexanes/EtOAc 2:1): 0.33; IR (neat) v = 3440, 2920, 1725, 1652, 1502, 1485, 1451, 1434, 1412, 1390, 1371, 1249, 1211, 1171, $1138 \mathrm{~cm}^{-1} ;{ }^{1} \mathbf{H}$ NMR $\left(500 \mathrm{MHz}, \mathrm{CDCl}_{3}\right) \delta 8.32(\mathrm{~d}, J=7.2 \mathrm{~Hz}, 2 \mathrm{H}), 7.64(\mathrm{t}, J$ $=7.4 \mathrm{~Hz}, 1 \mathrm{H}), 7.53(\mathrm{t}, J=7.7 \mathrm{~Hz}, 2 \mathrm{H}), 6.90(\mathrm{~d}, J=2.7 \mathrm{~Hz}, 1 \mathrm{H}), 6.79(\mathrm{~d}, J=3.0 \mathrm{~Hz}, 1 \mathrm{H}), 6.60(\mathrm{~s}, 1 \mathrm{H}), 6.17(\mathrm{~d}, J=2.7 \mathrm{~Hz}, 1 \mathrm{H})$, $5.96(\mathrm{~d}, J=1.3 \mathrm{~Hz}, 1 \mathrm{H}), 5.95(\mathrm{~d}, J=1.4 \mathrm{~Hz}, 1 \mathrm{H}), 4.98(\mathrm{~d}, J=10.0 \mathrm{~Hz}, 1 \mathrm{H}), 4.86(\mathrm{~s}, 1 \mathrm{H}), 4.63(\mathrm{~d}, J=10.0 \mathrm{~Hz}, 1 \mathrm{H}), 3.61(\mathrm{~s}, 3 \mathrm{H})$, $3.56(\mathrm{~s}, 3 \mathrm{H}), 2.39(\mathrm{qd}, J=6.8,3.3 \mathrm{~Hz}, 1 \mathrm{H}), 2.21(\mathrm{~s}, 3 \mathrm{H}), 1.38(\mathrm{~s}, 3 \mathrm{H}), 1.04(\mathrm{~d}, J=6.8 \mathrm{~Hz}, 3 \mathrm{H}) ;{ }^{13} \mathrm{C} \mathrm{NMR}(126 \mathrm{MHz}, \mathrm{CDCl}) \delta$ $170.3,169.3,165.8,150.0,149.7,142.8,134.0,130.7,129.2,128.9,128.8,127.9,120.8,118.6,101.8,99.5,97.9,78.71$, 78.69, 73.5, 70.5, 58.9, 54.3, 51.9, 45.5, 28.8, 21.4, 8.6; $[\alpha]^{23}{ }_{D}+41.3^{\circ},\left(\mathrm{c}=0.4, \mathrm{CH}_{2} \mathrm{Cl}_{2}\right)\left[\mathrm{lit}^{3}[\alpha]^{26}{ }_{\mathrm{D}}+62^{\circ},\left(\mathrm{c}=0.4, \mathrm{CH}_{2} \mathrm{Cl} 2\right)\right]$; HRMS: Calcd. for $\mathrm{C}_{31} \mathrm{H}_{30} \mathrm{O}_{13}[\mathrm{M}+\mathrm{K}]^{+}=649.1318 \mathrm{~m} / \mathrm{z}$, found $=649.1315 \mathrm{~m} / \mathrm{z}$.

\section{Compound 38}

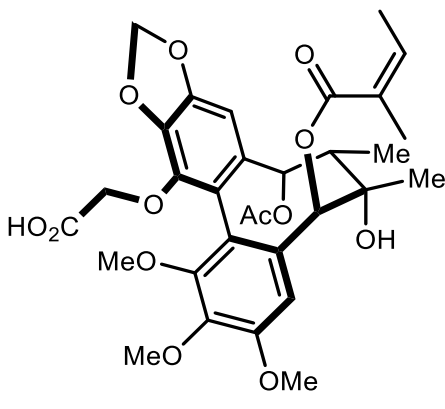

36

Chemical Formula: $\mathrm{C}_{31} \mathrm{H}_{36} \mathrm{O}_{13}$ Molecular Weight: 616.6160

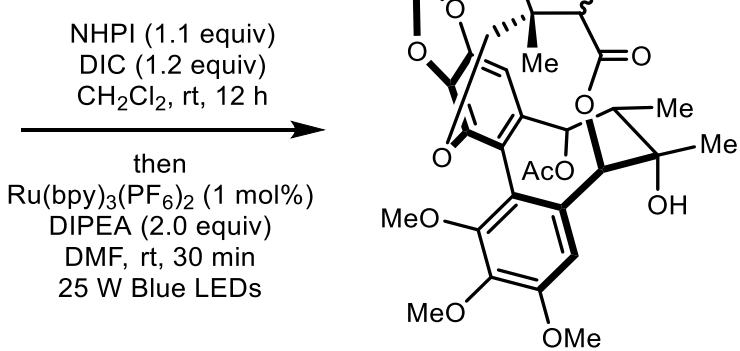

38, 30\%, 5:1 d.r.

Chemical Formula: $\mathrm{C}_{30} \mathrm{H}_{36} \mathrm{O}_{11}$ Molecular Weight: 572.6070

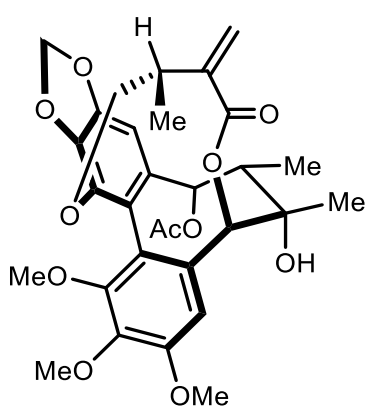

$39,27 \%$

Chemical Formula: $\mathrm{C}_{30} \mathrm{H}_{34} \mathrm{O}_{11}$ Molecular Weight: 570.5910

Procedure: A one-dram vial equipped with a Teflon-coated stir bar and a screw cap with septum was charged with 36 (0.020 mmol, $12.3 \mathrm{mg}, 1.0$ equiv) and $\mathrm{N}$-hydroxyphthalimide (NHPI, $0.022 \mathrm{mmol}, 3.6 \mathrm{mg}, 1.1$ equiv). The flask was evacuated and refill with $\mathrm{N}_{2}$ (repeat 3 times), prior to the addition of $\mathrm{CH}_{2} \mathrm{Cl}_{2}(0.2 \mathrm{~mL})$, followed by $N, N^{\prime}$ diisopropylcarbodiimide (DIC, $0.024 \mathrm{mmol}, 3.8 \mu \mathrm{L}, 1.2$ equiv) via microsyringe. The resulting mixture was stirred for $12 \mathrm{~h}$ at $\mathrm{rt}$ under $\mathrm{N}_{2}$. A solution of $\mathrm{Ru}(\mathrm{bpy})_{3}\left(\mathrm{PF}_{6}\right)_{2}(0.0002 \mathrm{mmol}, 0.2 \mathrm{mg}, 1.0 \mathrm{~mol} \%)$ and DIPEA (0.040 mmol, $\left.7.0 \mu \mathrm{L}, 2.0 \mathrm{equiv}\right)$ in DMF $(1.8 \mathrm{~mL})$ was then added. The resulting solution was then irradiated with $25 \mathrm{~W}$ blue LEDs at rt for $30 \mathrm{~min}$ with a cooling fan (See the picture above for the preparation of 3). It was then poured on a mixture of $\mathrm{H}_{2} \mathrm{O}(20 \mathrm{~mL})$ and $\mathrm{H}_{3} \mathrm{PO}_{4}(2$ $\mathrm{M}, 1 \mathrm{~mL})$, and extracted with EtOAc $(3 \times 20 \mathrm{~mL})$. The combined organic layers were washed with $\mathrm{H}_{2} \mathrm{O}(2 \times 20 \mathrm{~mL})$ and brine $(20 \mathrm{~mL})$, dried over $\mathrm{MgSO}_{4}$, filtered and concentrated in vacuo. The mixture was then analyzed by ${ }^{1} \mathrm{H}-\mathrm{NMR}$ with ethylene carbonate as the internal standard, showing $30 \%$ yield of 38 (5:1 d.r. mixture) and $27 \%$ yield of 39 . Analytically pure 38 was prepared using a different method, which will be described in a follow-up manuscript.

\section{Characterization:}

$\mathbf{R}_{f}=$ (hexanes/EtOAc 2:1): 0.30; IR (neat) $v=3573,2940,1732,1626,1595,1500,1478,1456,1430,1409,1367,1332$, $1251,1223,1195,1147,1106 \mathrm{~cm}^{-1} ;{ }^{1} \mathrm{H}$ NMR $\left(500 \mathrm{MHz}, \mathrm{CDCl}_{3}\right)$ major diastereomer $\delta 6.82(\mathrm{~s}, 1 \mathrm{H}), 6.49(\mathrm{~s}, 1 \mathrm{H}), 6.04(\mathrm{~d}, J=$ $1.1 \mathrm{~Hz}, 1 \mathrm{H}), 5.93(\mathrm{~d}, J=1.1 \mathrm{~Hz}, 1 \mathrm{H}), 5.85(\mathrm{~s}, 1 \mathrm{H}), 5.53(\mathrm{~s}, 1 \mathrm{H}), 3.92(\mathrm{~s}, 3 \mathrm{H}), 3.85(\mathrm{~s}, 3 \mathrm{H}), 3.79(\mathrm{dd}, J=12.4,4.0 \mathrm{~Hz}, 1 \mathrm{H}), 3.53$ $(\mathrm{s}, 3 \mathrm{H}), 3.50$ (dd, $J=12.5,6.5 \mathrm{~Hz}, 1 \mathrm{H}), 2.45(\mathrm{~s}, 1 \mathrm{H}), 2.35$ (qd, $J=7.1,4.7 \mathrm{~Hz}, 1 \mathrm{H}), 2.19-2.11(\mathrm{~m}, 1 \mathrm{H}), 1.98(\mathrm{q}, J=7.1 \mathrm{~Hz}$, $1 \mathrm{H}), 1.50$ (s, 3H), 1.32 (s, 3H), 1.28 (d, J=7.2 Hz, 3H), 0.98 (d, J=7.1 Hz, 3H), 0.90 (d, J = 7.3 Hz, 3H); ${ }^{13} \mathrm{C} \mathrm{NMR}(126 \mathrm{MHz}$, $\left.\mathrm{CDCl}_{3}\right)$ major diastereomer $\delta 174.9,168.8,152.3,151.6,148.2,141.8,139.1,138.6,132.9,131.7,122.15,122.07,111.0$, 103.0, 101.6, 85.2, 84.1, 73.8, 73.2, 60.75, 60.70, 56.3, 43.9, 40.8, 36.1, 28.4, 20.5, 17.8, 14.6, 12.7; $[\alpha]^{23} \mathrm{D}-3.9^{\circ},(\mathrm{c}=0.4$, $\left.\mathrm{CHCl}_{3}\right)$; HRMS: Calcd. for $\mathrm{C}_{30} \mathrm{H}_{36} \mathrm{O}_{11}[\mathrm{M}+\mathrm{Na}]^{+}=595.2150 \mathrm{~m} / \mathrm{z}$, found $=595.2138 \mathrm{~m} / \mathrm{z}$. 


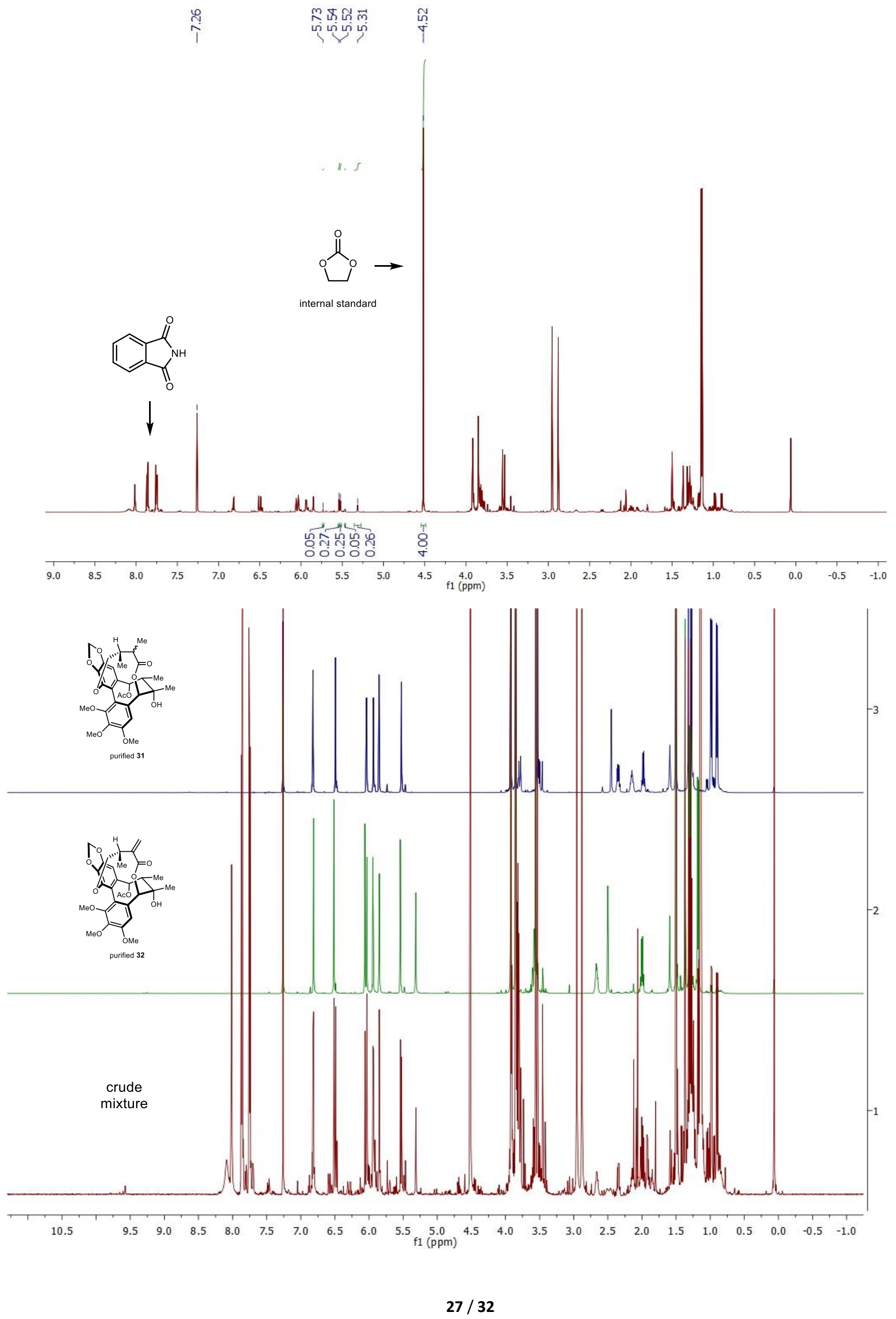




\section{Compound 39}

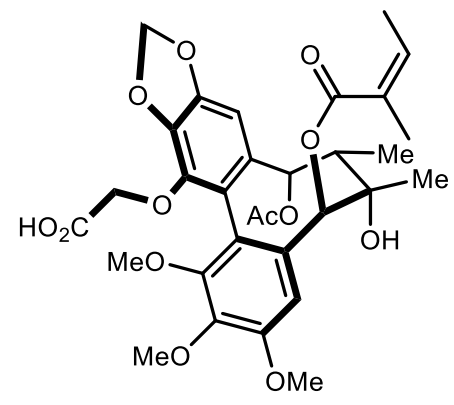

36

Chemical Formula: $\mathrm{C}_{31} \mathrm{H}_{36} \mathrm{O}_{13}$

Molecular Weight: 616.6160

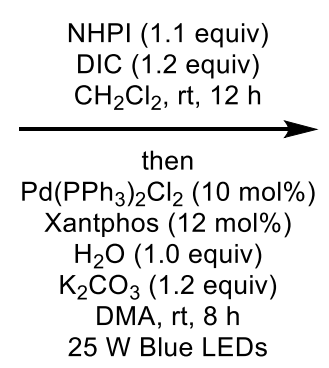

$53 \% 39+24 \% 36$

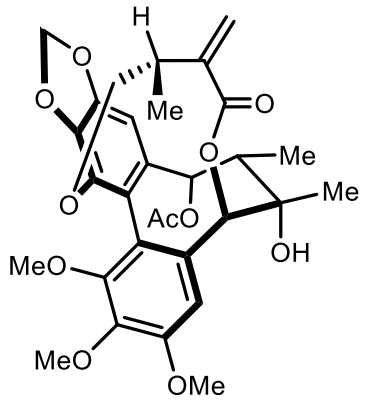

39

Chemical Formula: $\mathrm{C}_{30} \mathrm{H}_{34} \mathrm{O}_{11}$ Molecular Weight: 570.5910

Procedure: A $20 \mathrm{~mL}$ flame-dried test tube equipped with a Teflon-coated stir bar and a rubber septum was charged with $36(0.10 \mathrm{mmol}, 61.7 \mathrm{mg}, 1.0$ equiv) and $\mathrm{N}$-hydroxyphthalimide (NHPI, $0.11 \mathrm{mmol}, 17.9 \mathrm{mg}, 1.1$ equiv). The flask was evacuated and refill with $\mathrm{N}_{2}$ (repeat 3 times), prior to the addition of $\mathrm{CH}_{2} \mathrm{Cl}_{2}\left(1.0 \mathrm{~mL}\right.$ ), followed by $N, N^{\prime}$ diisopropylcarbodiimide (DIC, $0.12 \mathrm{mmol}, 18.8 \mu \mathrm{L}, 1.2$ equiv) via microsyringe. The resulting mixture was stirred for $12 \mathrm{~h}$ at rt under $\mathrm{N}_{2} . \mathrm{K}_{2} \mathrm{CO}_{3}\left(0.12 \mathrm{mmol}, 16.6 \mathrm{mg}, 1.2\right.$ equiv) was then added, the mixture was then purged with $\mathrm{N}_{2}$ for 5 min, and diluted by the addition of DMA ( $N, N$-dimethylaminoacetamide, $8 \mathrm{~mL})$. A solution of $\mathrm{Pd}\left(\mathrm{PPh}_{3}\right)_{2} \mathrm{Cl}_{2}(0.010 \mathrm{mmol}, 7.0$ $\mathrm{mg}, 10 \mathrm{~mol} \%)$, Xantphos ( $0.012 \mathrm{mmol}, 6.9 \mathrm{mg}, 12 \mathrm{~mol} \%)$ and $\mathrm{H}_{2} \mathrm{O}(0.10 \mathrm{mmol}, 1.8 \mu \mathrm{L}, 1.0$ equiv) in DMA (1 mL) was added. The resulting solution was then irradiated with $25 \mathrm{~W}$ blue LEDs at $\mathrm{rt}$ for $8 \mathrm{~h}$ with a cooling fan (See the picture above for the preparation of 3). It was then poured on a mixture of $\mathrm{H}_{2} \mathrm{O}(50 \mathrm{~mL})$ and $\mathrm{H}_{3} \mathrm{PO}_{4}(2 \mathrm{M}, 1 \mathrm{~mL})$, and extracted with EtOAc $(3 \times 50 \mathrm{~mL})$. The combined organic layers were washed with $\mathrm{H}_{2} \mathrm{O}(2 \times 50 \mathrm{~mL})$ and brine $(20 \mathrm{~mL})$, dried over $\mathrm{MgSO}_{4}$, filtered and concentrated in vacuo. The crude product was purified on silica gel (5:1 to 2:1 hexanes/EtOAc) to provide the 39 (30.2 $\mathrm{mg}, 0.0527 \mathrm{mmol}, 53 \%)$ as an off-white foam. Starting material $36(14.8 \mathrm{mg}, 0.0240 \mathrm{mmol}, 24 \%)$ was also recovered.

\section{Characterization:}

$\mathbf{R}_{f}=$ (hexanes/EtOAc 2:1): 0.30; IR (neat) $v=3567,2942,1746,1723,1677,1628,1593,1500,1479,1458,1430,1408$, 1367, 1331, 1294, 1221, 1196, 1151, $1105 \mathrm{~cm}^{-1}$; ${ }^{1} \mathrm{H}$ NMR (500 MHz, CDCl $) \delta 6.81(\mathrm{~s}, 1 \mathrm{H}), 6.51(\mathrm{~s}, 1 \mathrm{H}), 6.06(\mathrm{~s}, 1 \mathrm{H}), 6.03$ $(\mathrm{d}, J=1.2 \mathrm{~Hz}, 1 \mathrm{H}$ ), $5.94(\mathrm{~d}, J=1.2 \mathrm{~Hz}, 1 \mathrm{H}), 5.85(\mathrm{~s}, 1 \mathrm{H}), 5.54(\mathrm{~s}, 1 \mathrm{H}), 5.32(\mathrm{~s}, 1 \mathrm{H}), 3.92(\mathrm{~s}, 3 \mathrm{H}), 3.85(\mathrm{~s}, 3 \mathrm{H}), 3.59$ (dd, $J=11.7$, $4.3 \mathrm{~Hz}, 1 \mathrm{H}$ ), $3.56(\mathrm{~s}, 3 \mathrm{H}), 3.54(\mathrm{dd}, J=11.7,6.3 \mathrm{~Hz}, 1 \mathrm{H}), 2.70-2.63(\mathrm{~m}, 1 \mathrm{H}), 2.50(\mathrm{~s}, 1 \mathrm{H}), 2.00(\mathrm{q}, J=7.2 \mathrm{~Hz}, 1 \mathrm{H}), 1.50(\mathrm{~s}$, 3H), $1.37(\mathrm{~s}, 3 \mathrm{H}), 1.29$ (d, $J=7.2 \mathrm{~Hz}, 3 \mathrm{H}), 1.18(\mathrm{~d}, J=6.9 \mathrm{~Hz}, 3 \mathrm{H}) ;{ }^{13} \mathrm{C}$ NMR $\left(126 \mathrm{MHz}, \mathrm{CDCl}_{3}\right) \delta$ 168.7, 168.0, 152.3, 151.8, 148.2, 143.3, 142.0, 140.5, 138.2, 133.2, 131.1, 123.0, 122.33, 122.26, 111.3, 103.9, 101.7, 85.9, 84.2, 74.8, 74.0, 60.8, $60.7,56.3,43.7,35.7,28.2,20.5,17.8,16.3 ;[\alpha]^{23}{ }_{D}+3.7^{\circ},\left(c=1.0, \mathrm{CHCl}_{3}\right)$; HRMS: Calcd. for $\mathrm{C}_{30} \mathrm{H}_{34} \mathrm{O}_{11}[\mathrm{M}+\mathrm{Na}]^{+}=593.1993$ $\mathrm{m} / \mathrm{z}$, found $=593.2006 \mathrm{~m} / \mathrm{z}$

\section{Compounds 4 (kadsuphilin N) and SI-11}

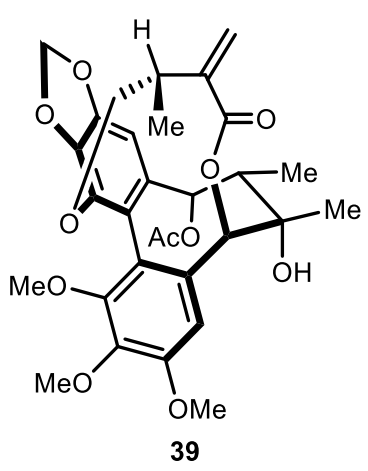

Chemical Formula: $\mathrm{C}_{30} \mathrm{H}_{34} \mathrm{O}_{11}$ Molecular Weight: 570.5910

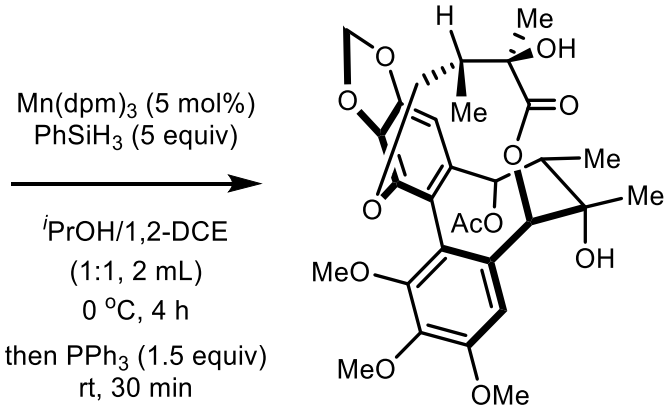

$78 \%, 5: 1$ d.r. kadsuphilin $\mathrm{N}(4), 65 \%$ Chemical Formula: $\mathrm{C}_{30} \mathrm{H}_{36} \mathrm{O}_{12}$ Molecular Weight: 588.6060

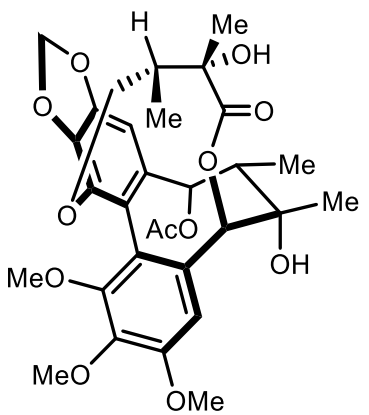

SI-11, 13\%

Chemical Formula: $\mathrm{C}_{30} \mathrm{H}_{36} \mathrm{O}_{12}$ Molecular Weight: 588.6060 
Procedure: A $20 \mathrm{~mL}$ flame-dried Radley tube equipped with a Teflon-coated stir bar and a sealed cap was charged with 39 (0.091 mmol, $51.9 \mathrm{mg}, 1.0$ equiv) and $\mathrm{Mn}(\mathrm{dpm})_{3}(0.0045 \mathrm{mmol}, 2.8 \mathrm{mg}, 5 \mathrm{~mol} \%)$, and dissolved in ${ }^{i} \mathrm{PrOH}(1 \mathrm{~mL})$ and 1,2-DCE (1 mL). The mixture was cooled to $0{ }^{\circ} \mathrm{C}$, at which point $\mathrm{PhSiH}_{3}(0.455 \mathrm{mmol}, 56.1 \mu \mathrm{L}, 5.0$ equiv) was added via micro-syringe. The resulting mixture was connected to a tank of $\mathrm{O}_{2}$ and pressurized to $1 \mathrm{~atm}$. Under a constant pressure of $\mathrm{O}_{2}(1 \mathrm{~atm})$, the reaction was vented 3 times for $10 \mathrm{~s}$ to remove $\mathrm{N}_{2}$, and stirred for $4 \mathrm{~h}$ at $0{ }^{\circ} \mathrm{C}$ under $\mathrm{O}_{2}(1 \mathrm{~atm})$. It was then depressurized, and $\mathrm{PPh}_{3}(0.137 \mathrm{mmol}, 35.9 \mathrm{mg}, 1.5$ equiv) was added as solid, and the resulting mixture was stirred at $\mathrm{rt}$ for another $30 \mathrm{~min}$. It was then poured on $\mathrm{Na}_{2} \mathrm{~S}_{2} \mathrm{O}_{3}(20 \mathrm{~mL})$ and extracted with $\mathrm{CH}_{2} \mathrm{Cl}_{2}(3 \times 20 \mathrm{~mL})$. The combined organic layers were dried over $\mathrm{MgSO}_{4}$, filtered and concentrated in vacuo. The crude product was purified on silica gel (2:1 to 1:1 hexanes/EtOAC) to provide a 5:1 d.r. mixture of the product, which was further separated by preparatory TLC using $2 \% \mathrm{MeOH} / \mathrm{CHCl}_{3}$ as the eluent to afford $4(35.0 \mathrm{mg}, 0.0595 \mathrm{mmol}, 65 \%)$ as an off-white foam, and $\mathbf{S I - 1 1}(7.0 \mathrm{mg}$, $0.0119 \mathrm{mmol}, 13 \%)$ as a pale-yellow oil.

\section{Characterization:}

Major diastereomer, kadsuphilin $\mathbf{N}: \mathbf{R}_{\boldsymbol{f}}=$ (hexanes/EtOAc 1:1): 0.32; IR (neat) $\mathbf{v}=3565,3490,2942,1734,1624,1595$, 1500, 1477, 1457, 1431, 1410, 1367, 1334, 1249, 1221, 1196, 1149, $1104 \mathrm{~cm}^{-1} ;{ }^{1} \mathrm{H}$ NMR (500 MHz, CDCl $) \delta 6.84(\mathrm{~s}, 1 \mathrm{H})$, $6.48(\mathrm{~s}, 1 \mathrm{H}), 6.03(\mathrm{~d}, J=1.1 \mathrm{~Hz}, 1 \mathrm{H}), 5.94(\mathrm{~d}, J=1.0 \mathrm{~Hz}, 1 \mathrm{H}), 5.76(\mathrm{~s}, 1 \mathrm{H}), 5.48(\mathrm{~s}, 1 \mathrm{H}), 4.17(\mathrm{dd}, J=12.4,4.3 \mathrm{~Hz}, 1 \mathrm{H}), 3.92$ $(\mathrm{s}, 3 \mathrm{H}), 3.84(\mathrm{~s}, 3 \mathrm{H}), 3.63(\mathrm{dd}, J=12.4,4.5 \mathrm{~Hz}, 1 \mathrm{H}), 3.46(\mathrm{~s}, 3 \mathrm{H}), 2.59(\mathrm{~s}, 1 \mathrm{H}), 1.97(\mathrm{q}, J=7.2 \mathrm{~Hz}, 1 \mathrm{H}), 1.96-1.88(\mathrm{~m}, 1 \mathrm{H})$, $1.80(\mathrm{~s}, 1 \mathrm{H}), 1.49(\mathrm{~s}, 3 \mathrm{H}), 1.34(\mathrm{~s}, 3 \mathrm{H}), 1.30(\mathrm{~d}, J=7.2 \mathrm{~Hz}, 3 \mathrm{H}), 1.24(\mathrm{~s}, 3 \mathrm{H}), 0.97$ (d, $J=7.2 \mathrm{~Hz}, 3 \mathrm{H}) ;{ }^{13} \mathrm{C} \mathrm{NMR}(126 \mathrm{MHz}$, $\left.\mathrm{CDCl}_{3}\right) \delta 172.5,168.8,152.4,151.6,148.7,141.9,139.1,137.6,132.9,130.9,121.8,121.3,111.0,102.6,101.7,86.7,84.0$, $76.8,73.9,72.5,60.8,60.6,56.3,44.2,42.6,28.5,21.5,20.4,18.0,12.9 ;[\alpha]^{23}{ }_{\mathrm{D}}+24.9^{\circ},\left(\mathrm{c}=1.0, \mathrm{CH}_{2} \mathrm{Cl}_{2}\right)\left[{ }^{2}{ }^{2}{ }^{2}{ }^{2}[\alpha]^{26} \mathrm{D}-2.4^{\circ}\right.$, $\left.\left(\mathrm{CH}_{2} \mathrm{Cl}_{2}\right)\right]$; HRMS: Calcd. for $\mathrm{C}_{30} \mathrm{H}_{36} \mathrm{O}_{12}[\mathrm{M}+\mathrm{Na}]^{+}=611.2099 \mathrm{~m} / \mathrm{z}$, found $=611.2102 \mathrm{~m} / \mathrm{z}$.

Minor diastereomer: $\mathbf{R}_{f}=($ hexanes/EtOAc 1:1): 0.33; IR (neat) $v=3563,3507,2919,1732,1622,1596,1501,1471,1457$, 1432, 1411, 1369, 1335, 1223, 1177, 1148, $1105 \mathrm{~cm}^{-1} ;{ }^{1} \mathrm{H}$ NMR (500 MHz, CDCl $) \delta 6.85$ (s, 1H), 6.47 (s, 1H), 6.04 (d, $J=$ $1.3 \mathrm{~Hz}, 1 \mathrm{H}), 5.93(\mathrm{~d}, J=1.3 \mathrm{~Hz}, 1 \mathrm{H}), 5.71(\mathrm{~s}, 1 \mathrm{H}), 5.46(\mathrm{~s}, 1 \mathrm{H}), 4.03(\mathrm{dd}, J=12.2,4.5 \mathrm{~Hz}, 1 \mathrm{H}), 3.94(\mathrm{~s}, 3 \mathrm{H}), 3.86(\mathrm{~s}, 3 \mathrm{H}), 3.73$ $(\mathrm{dd}, J=12.2,6.0 \mathrm{~Hz}, 1 \mathrm{H}), 3.46(\mathrm{~s}, 3 \mathrm{H}), 3.22(\mathrm{~s}, 1 \mathrm{H}), 2.62(\mathrm{~s}, 1 \mathrm{H}), 1.90(\mathrm{q}, J=7.2 \mathrm{~Hz}, 1 \mathrm{H}), 1.86-1.77(\mathrm{~m}, 1 \mathrm{H}), 1.50(\mathrm{~s}, 3 \mathrm{H})$, $1.32(\mathrm{~s}, 3 \mathrm{H}), 1.30(\mathrm{~d}, J=7.2 \mathrm{~Hz}, 3 \mathrm{H}), 1.25(\mathrm{~s}, 3 \mathrm{H}), 1.02(\mathrm{~d}, J=7.1 \mathrm{~Hz}, 3 \mathrm{H}) ;{ }^{13} \mathrm{C} \mathrm{NMR}\left(126 \mathrm{MHz}, \mathrm{CDCl}_{3}\right) \delta 177.7,168.8,152.5$, 151.6, 148.6, 142.0, 138.6, 137.7, 133.1, 130.5, 121.9, 121.4, 110.8, 102.2, 101.6, 88.4, 83.9, 74.4, 74.0, 72.6, 60.8, 60.6, $56.3,44.5,37.7,28.6,25.2,20.4,18.1,11.5 ;[\alpha]^{23}{ }_{D}-5.5^{\circ},\left(\mathrm{c}=0.6, \mathrm{CHCl}_{3}\right)$; HRMS: Calcd. for $\mathrm{C}_{30} \mathrm{H}_{36} \mathrm{O}_{12}[\mathrm{M}+\mathrm{Na}]^{+}=611.2099$ $\mathrm{m} / \mathrm{z}$, found $=611.2112 \mathrm{~m} / \mathrm{z}$. 


\section{X-ray Crystallographic Data}

\section{General Experimental}

A suitable crystal was selected and mounted on a Cryoloop on a Bruker Venture Metaljet diffractometer. The crystal was kept at $150 \mathrm{~K}$ during data collection. Using Olex $2,^{12}$ the structure was solved with the $\mathrm{XT}^{13}$ structure solution program using Intrinsic Phasing and refined with the $\mathrm{XL}^{14}$ refinement package using Least Squares minimization.
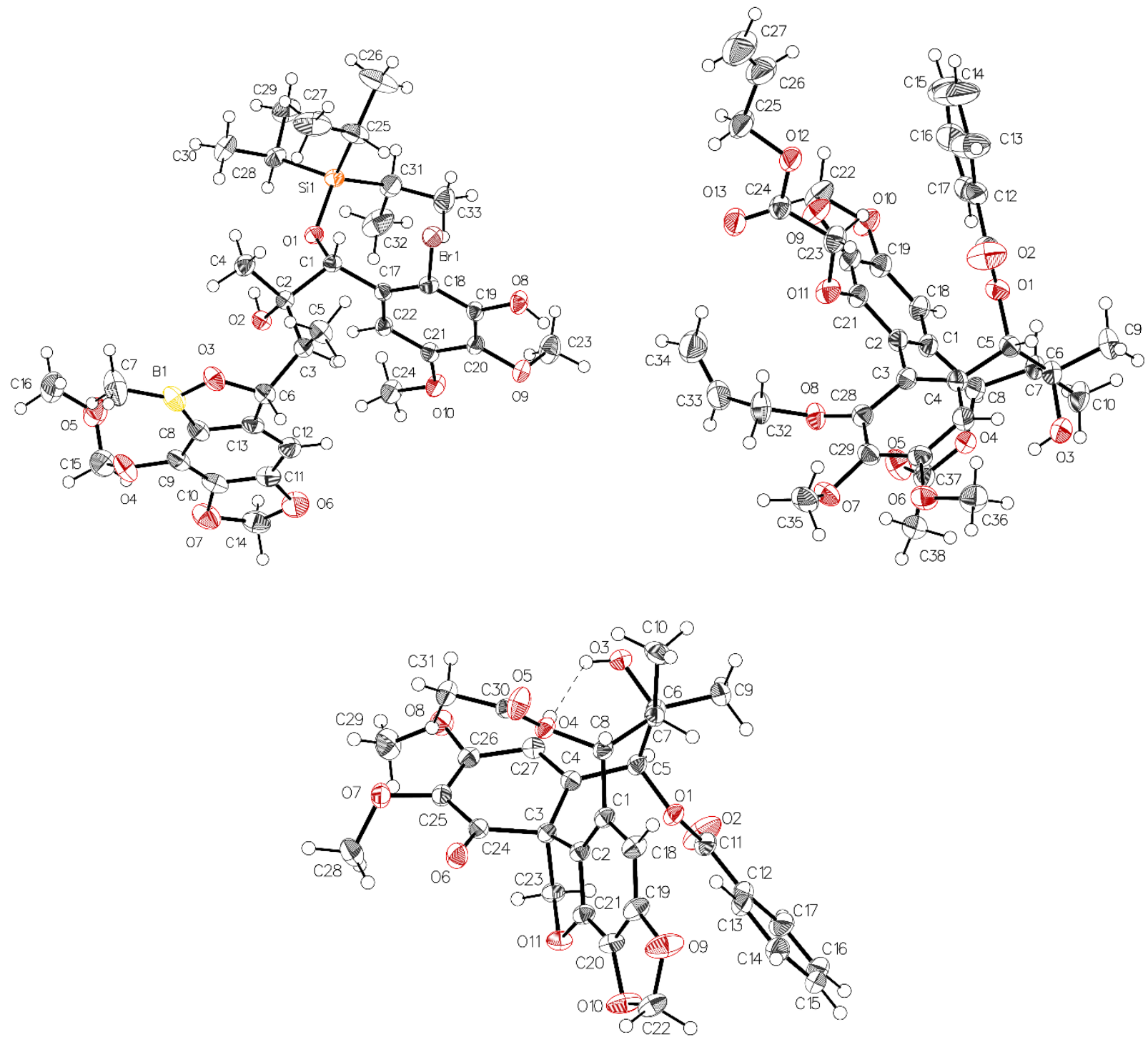

Figure S1. ORTEP representations of Compounds 29 (top left), SI-8 (top right), $\mathbf{3}$ (bottom). 
Table S5. Crystal data and structure refinement for compounds 29, SI-8 and 3.

\begin{tabular}{|c|c|c|c|}
\hline Identification code & Compound 29 & Compound SI-8 & Compound 3 \\
\hline Empirical formula & $\mathrm{C}_{33} \mathrm{H}_{50} \mathrm{BBrO}_{10} \mathrm{Si}$ & $\mathrm{C}_{38} \mathrm{H}_{40} \mathrm{O}_{13}$ & $\mathrm{C}_{31} \mathrm{H}_{30} \mathrm{O}_{11}$ \\
\hline Formula weight & 725.54 & 704.70 & 578.55 \\
\hline Temperature/K & 150 & 150 & 150 \\
\hline Crystal system & orthorhombic & orthorhombic & monoclinic \\
\hline Space group & $\mathrm{P} 22_{1} 2_{1} 2_{1}$ & $\mathrm{P} 22_{1} 2_{1} 2_{1}$ & $\mathrm{P} 2_{1}$ \\
\hline$a / \AA ̊$ & $13.7600(4)$ & $9.4729(3)$ & 11.9339(7) \\
\hline$b / \AA ̊$ & $13.7821(4)$ & $18.1648(6)$ & $8.9903(5)$ \\
\hline$c / A ̊$ & $19.4436(5)$ & $20.2360(7)$ & $12.6917(7)$ \\
\hline$\alpha /^{\circ}$ & 90 & 90 & 90 \\
\hline$\beta /^{\circ}$ & 90 & 90 & $93.929(2)$ \\
\hline$\gamma /{ }^{\circ}$ & 90 & 90 & 90 \\
\hline Volume $/ \AA^{3}$ & $3687.32(18)$ & $3482.1(2)$ & 1358.48(13) \\
\hline Z & 4 & 4 & 2 \\
\hline$\rho_{\text {calc }} \mathrm{g} / \mathrm{cm}^{3}$ & 1.307 & 1.344 & 1.414 \\
\hline$\mu / \mathrm{mm}^{-1}$ & 1.493 & 0.541 & 0.576 \\
\hline$F(000)$ & 1528.0 & 1488.0 & 608.0 \\
\hline Crystal size $/ \mathrm{mm}^{3}$ & $0.25 \times 0.06 \times 0.05$ & $0.4 \times 0.32 \times 0.27$ & $0.24 \times 0.24 \times 0.19$ \\
\hline Radiation & GaK $\alpha$ & GaKa & GaKa \\
\hline Kduldtion & $(\lambda=1.34139)$ & $(\lambda=1.34139)$ & $(\lambda=1.34139)$ \\
\hline $\begin{array}{c}2 \Theta \text { range for } \\
\text { data collection } /^{\circ}\end{array}$ & 6.846 to 121.408 & 5.688 to 110.178 & 6.072 to 121.416 \\
\hline \multirow{3}{*}{ Index ranges } & $-17 \leq h \leq 17$ & $-10 \leq h \leq 10$ & $-15 \leq h \leq 15$ \\
\hline & $-17 \leq k \leq 17$ & $-22 \leq k \leq 22$ & $-11 \leq k \leq 11$ \\
\hline & $-24 \leq 1 \leq 25$ & $-24 \leq 1 \leq 24$ & $-16 \leq 1 \leq 16$ \\
\hline Reflections collected & 48391 & 29780 & 37181 \\
\hline \multirow{3}{*}{ Independent reflections } & 8458 & 6514 & 6241 \\
\hline & {$\left[R_{\text {int }}=0.0310\right.$} & {$\left[R_{\text {int }}=0.0349\right.$} & {$\left[R_{\text {int }}=0.0257\right.$} \\
\hline & $\left.R_{\text {sigma }}=0.0195\right]$ & $\left.\mathrm{R}_{\text {sigma }}=0.0221\right]$ & $\left.\mathrm{R}_{\text {sigma }}=0.0174\right]$ \\
\hline Data/restraints/parameters & $8458 / 3 / 436$ & $6514 / 0 / 478$ & $6241 / 1 / 386$ \\
\hline Goodness-of-fit on $F^{2}$ & 1.080 & 1.099 & 1.150 \\
\hline \multirow{2}{*}{ Final R indexes $[\mid>=2 \sigma(I)]$} & $\mathrm{R}_{1}=0.0263$ & $\mathrm{R}_{1}=0.0353$ & $\mathrm{R}_{1}=0.0398$ \\
\hline & $w R_{2}=0.0682$ & $w R_{2}=0.0861$ & $w R_{2}=0.0933$ \\
\hline \multirow{2}{*}{ Final $R$ indexes [all data] } & $\mathrm{R}_{1}=0.0273$ & $\mathrm{R}_{1}=0.0358$ & $\mathrm{R}_{1}=0.0399$ \\
\hline & $w R_{2}=0.0691$ & $w R_{2}=0.0867$ & $w R_{2}=0.0934$ \\
\hline $\begin{array}{l}\text { Largest diff. peak/ } \\
\text { hole / e } \AA^{-3}\end{array}$ & $0.49 /-0.23$ & $0.32 /-0.25$ & $0.28 /-0.43$ \\
\hline Flack parameter & $-0.037(4)$ & $-0.04(4)$ & $-0.02(5)$ \\
\hline
\end{tabular}




\section{References and Notes}

1. Xu, L.-J.; Peng, Y.; Chen, S.-B.; Chen, S.-L.; Xiao, P.-G. Four New Lignans from Kadsura heteroclita. Heterocycles 2007, 71, 941-947.

2. Lin, Y.-C.; Cheng, Y.-B.; Liaw, C.-C.; Lo, I.-W.; Kuo, Y.-H.; Chiang, M. Y.; Chou, C.-H.; Shen, Y.-C. New Lignans from the Leaves and Stems of Kadsura philippinensis. Molecules 2013, 18, 6573-6583.

3. Shen, Y.-C.; Lin, Y.-C.; Cheng, Y.-B.; Kuo, Y.-H.; Liaw, C.-C. Taiwankadsurins A, B, and C, Three New C19 Homolignans from Kadsura philippinensis. Org. Lett. 2005, 7, 5297-5300.

4. Singh, S. B.; Pettit, G. R. Antineoplastic agents. 166. Isolation, Structure, and Synthesis of Combretastatin C-1. J. Org. Chem. 1989, 54, 4105-4114.

5. Belokon, Y. N.; Clegg, W.; Harrington, R. W.; Maleev, V. I.; North, M.; Pujol, M. O.; Usanov, D. L.; Young, C. MechanismGuided Development of VO(salen)X Complexes as Catalysts for the Asymmetric Synthesis of Cyanohydrin Trimethylsilyl Ethers. Chem. Eur. J. 2009, 15, 2148-2165.

6. Van Draanen, N. A.; Arseniyadis, S.; Crimmins, M. T.; Heathcock, C. H. Protocols for the Preparation of Each of the Four Possible Stereoisomeric .alpha.-alkyl-.beta.-Hydroxy Carboxylic Acids from a Single Chiral Aldol Reagent. J. Org. Chem. 1991, 56, 2499-2506.

7. Bartoli, G.; Bosco, M.; Di Martino, E.; Marcantoni, E.; Sambri, L. Highly Stereoselective and Efficient Addition of Organocerium Reagents to syn- $\beta$-Alkyl- $\beta$-hydroxy- $\alpha$-methyl Ketones by Way of Their Titanium Alkoxides - Synthesis of Complex 1,3-Diol Units with Three Stereodefined Centres. Eur. J. Org. Chem. 2001, 2001, 2901-2909.

8. Hoye, T. R.; Jeon, J.; Kopel, L. C.; Ryba, T. D.; Tennakoon, M. A.; Wang, Y. Total Synthesis of Peloruside A through Kinetic Lactonization and Relay Ring-Closing Metathesis Cyclization Reactions. Angew. Chem. Int. Ed. 2010, 49, 6151-6155.

9. Ramesh, C.; Ravindranath, N.; Das, B. Simple, Efficient, and Selective Deprotection of Phenolic Methoxymethyl Ethers Using Silica-Supported Sodium Hydrogen Sulfate as a Heterogeneous Catalyst1. J. Org. Chem. 2003, 68, 7101-7103.

10. Liang, X.; Grue-Sørensen, G.; Petersen, A. K.; Högberg, T. Semisynthesis of Ingenol 3-Angelate (PEP005): Efficient Stereoconservative Angeloylation of Alcohols. Synlett 2012, 23, 2647-2652.

11. Nicolaou, K. C.; Dalby, S. M.; Majumder, U. A Concise Asymmetric Total Synthesis of Aspidophytine. J. Am. Chem. Soc. 2008, 130, 14942-14943.

12. Dolomanov, O. V.; Bourhis, L. J.; Gildea, R. J.; Howard, J. A. K.; Puschmann, H. OLEX2: a Complete Structure Solution, Refinement and Analysis Program. J. Appl. Cryst. 2009, 42, 339-341.

13. Sheldrick, G. SHELXT - Integrated Space-Group and Crystal-Structure Determination. Acta Cryst. A 2015, 71, 3-8.

14. Sheldrick, G. Crystal Structure Refinement with SHELXL. Acta Cryst. C 2015, 71, 3-8. 


\section{Mimicking Noncanonical Oxidations with Redox-Neutral Photocatalysis}

Zheng Huang and Jean-Philip Lumb*

Department of Chemistry, McGill University, Montreal, QC H3A OB8, Canada.

*email : jean-philip.lumb@mcgill.ca

Analytical Data 
${ }^{1} \mathrm{H}-\mathrm{NMR}$ of compound 3 (heteroclitin J)

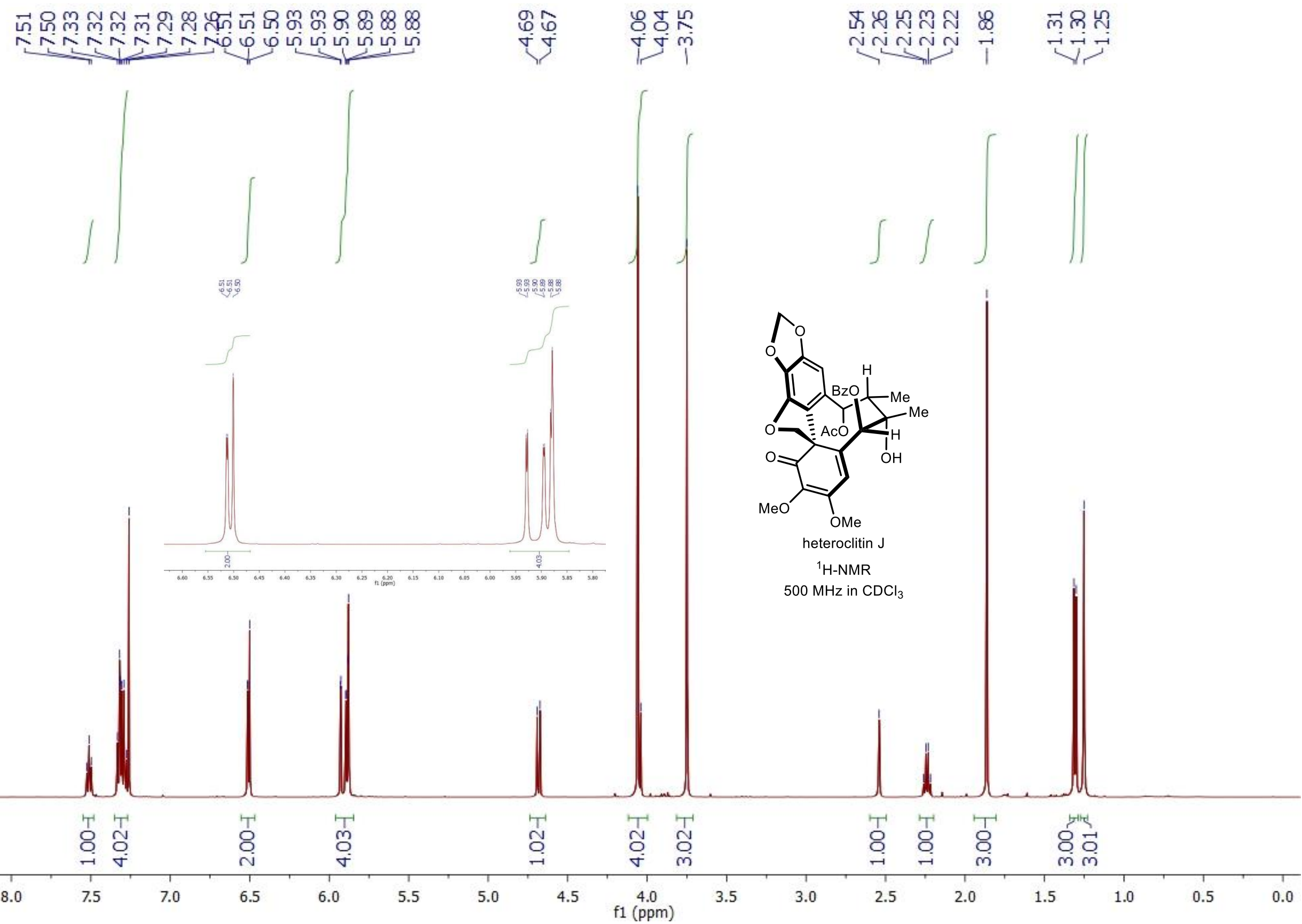

$2 / 77$ 
${ }^{13} \mathrm{C}-\mathrm{NMR}$ of compound 3 (heteroclitin J)

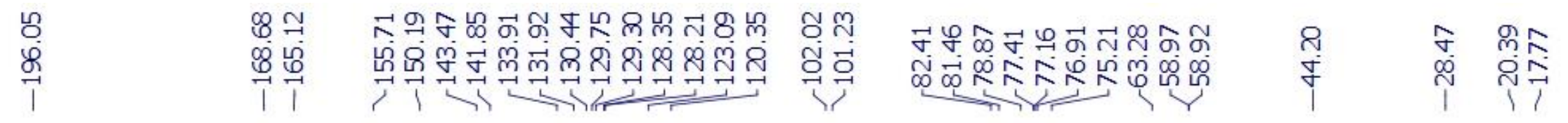
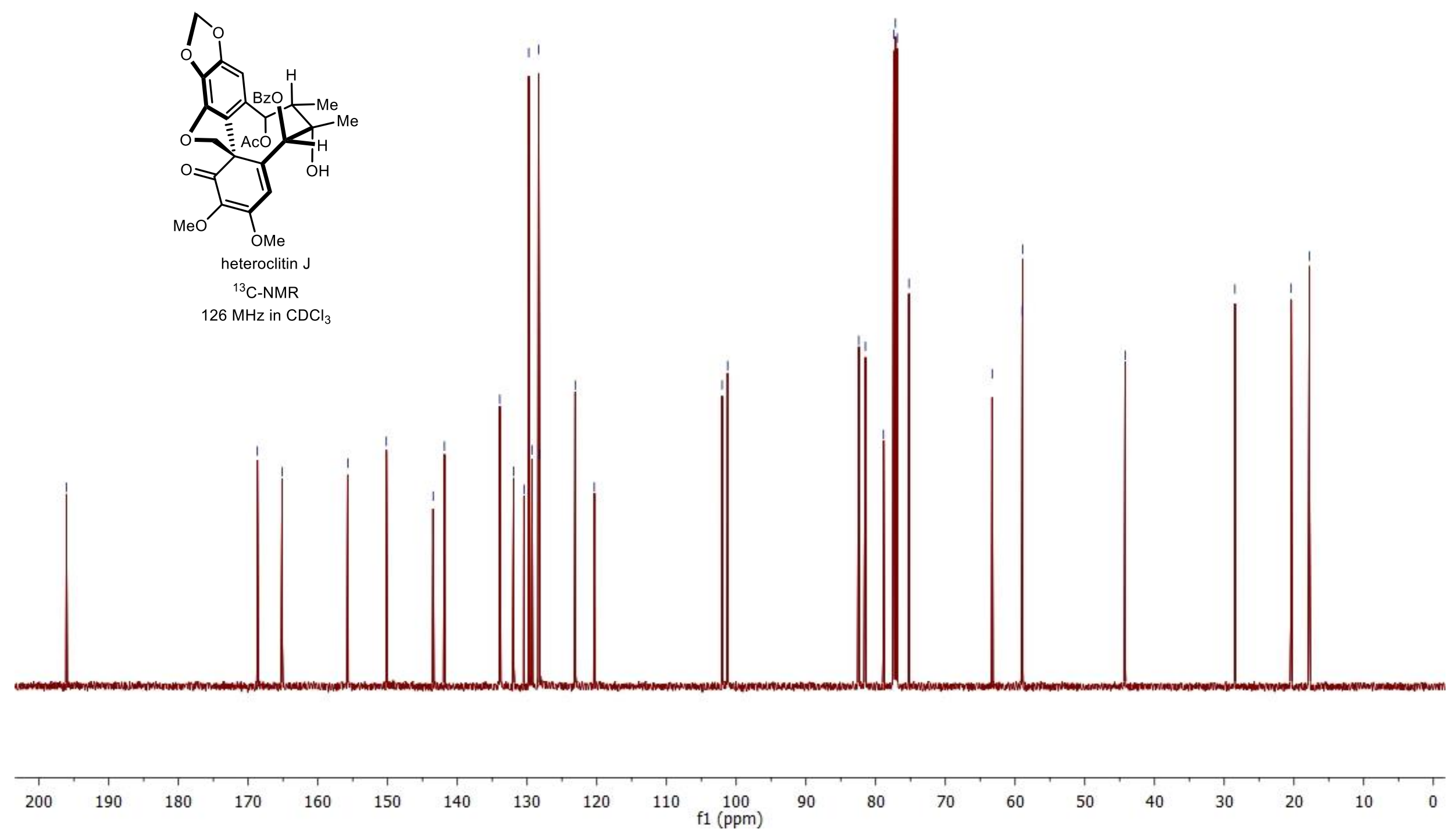
${ }^{1} \mathrm{H},{ }^{1} \mathrm{H}-\mathrm{COSY}$ of compound 3 (heteroclitin J)

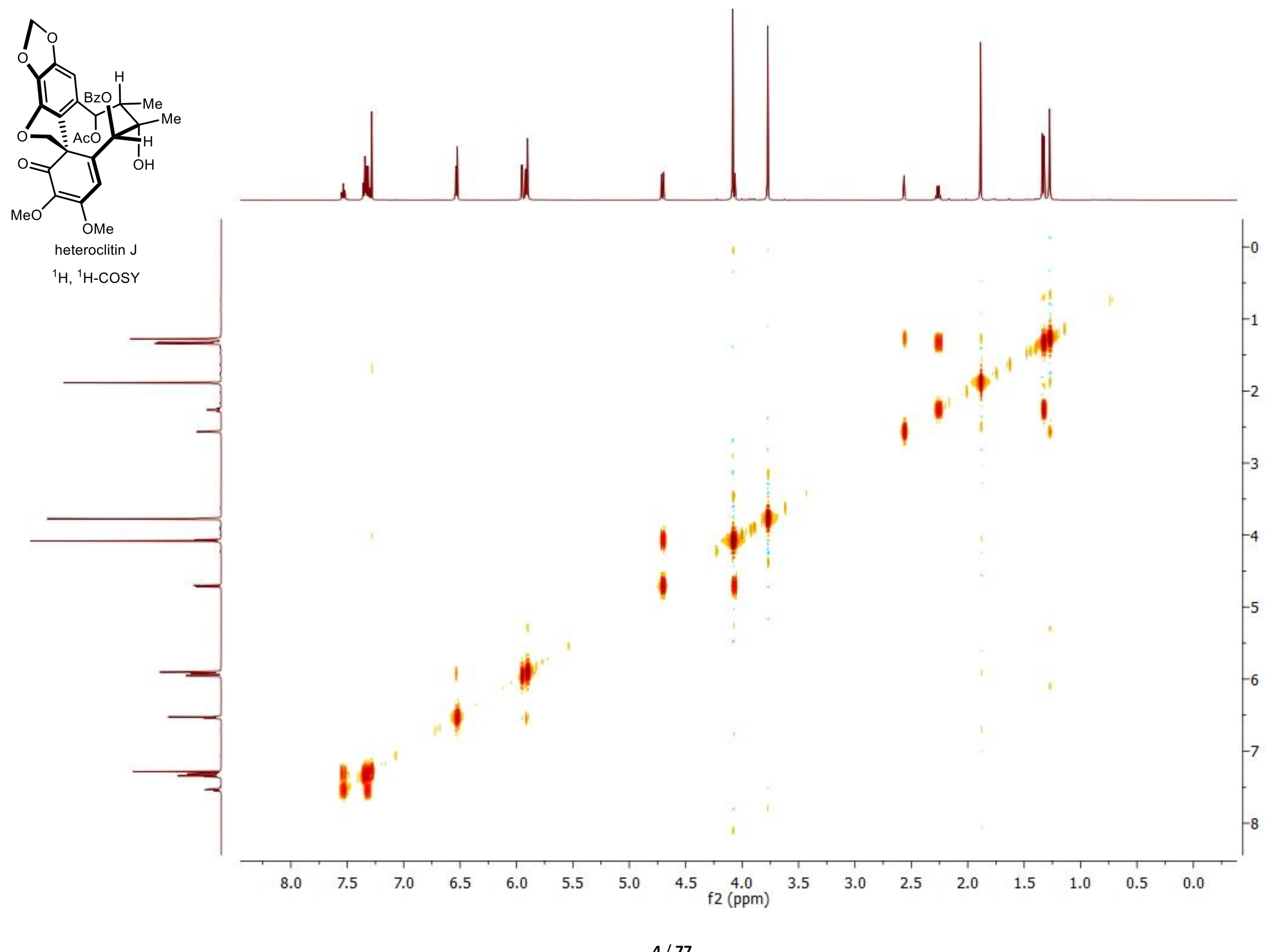


${ }^{1} \mathrm{H},{ }^{1} \mathrm{H}-\mathrm{NOESY}$ of compound 3 (heteroclitin J)

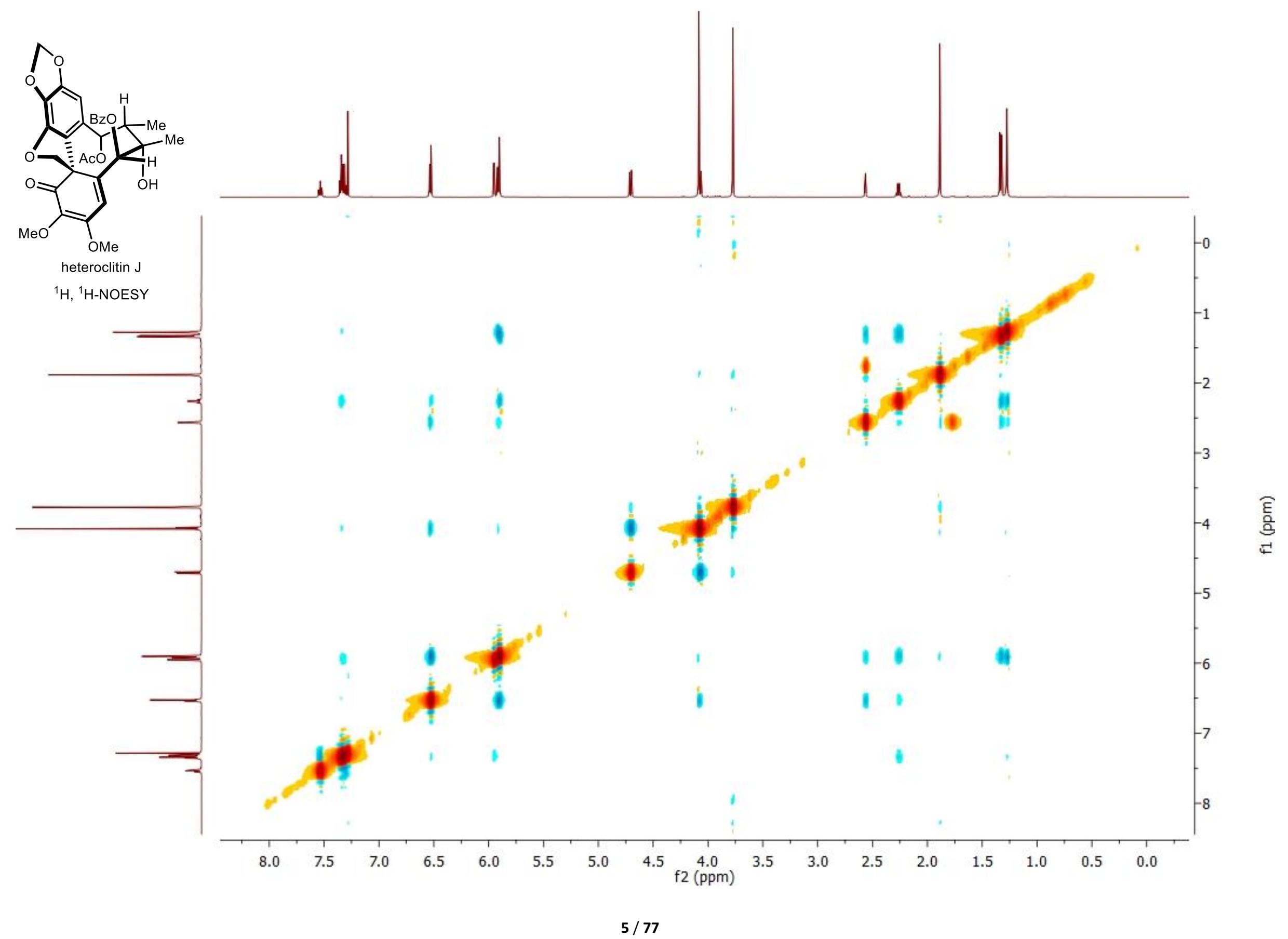


${ }^{1} \mathrm{H},{ }^{13} \mathrm{C}-\mathrm{HSQC}$ of compound 3 (heteroclitin J)

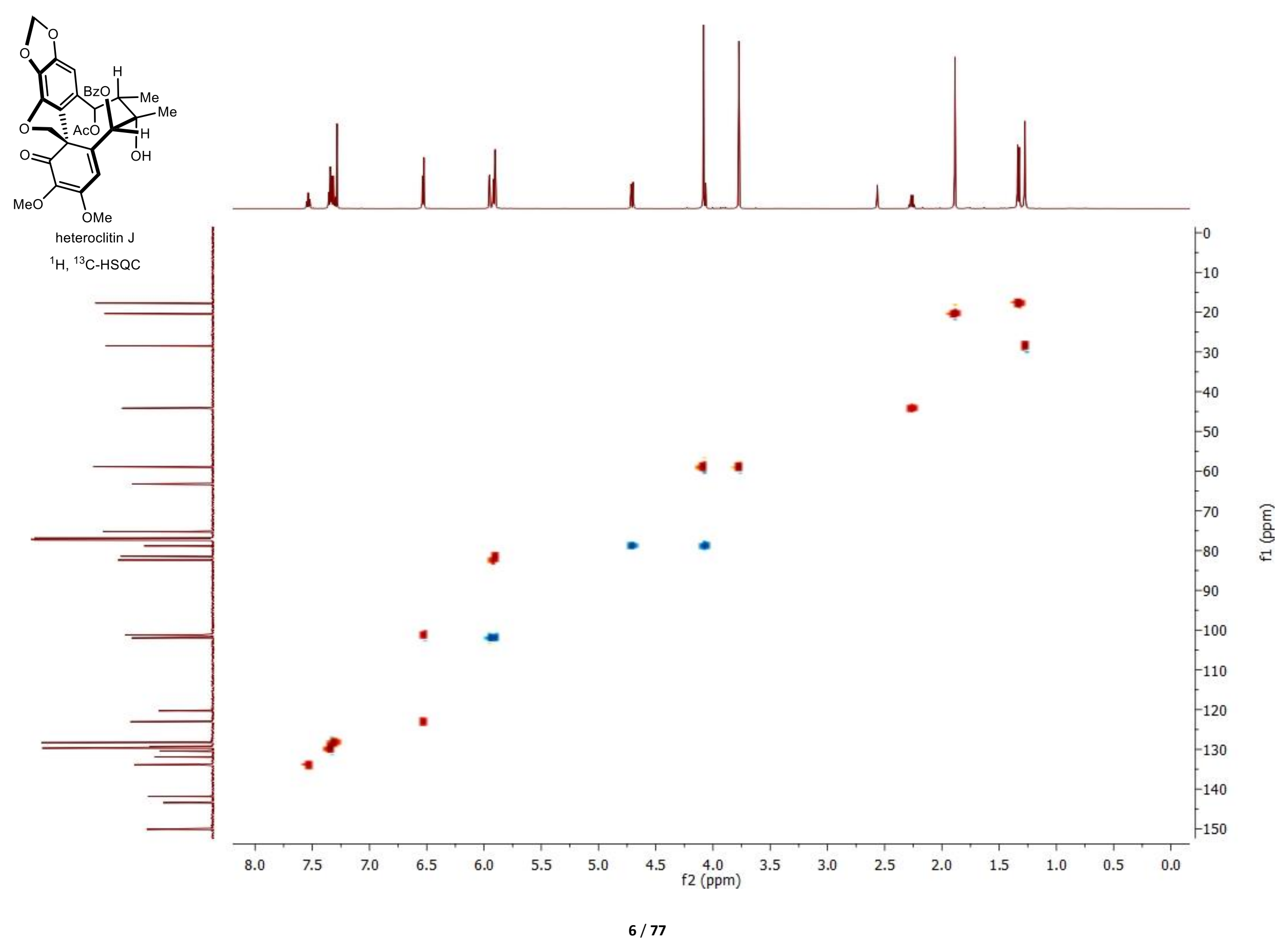


${ }^{1} \mathrm{H},{ }^{13} \mathrm{C}-\mathrm{HMBC}$ of compound 3 (heteroclitin J)

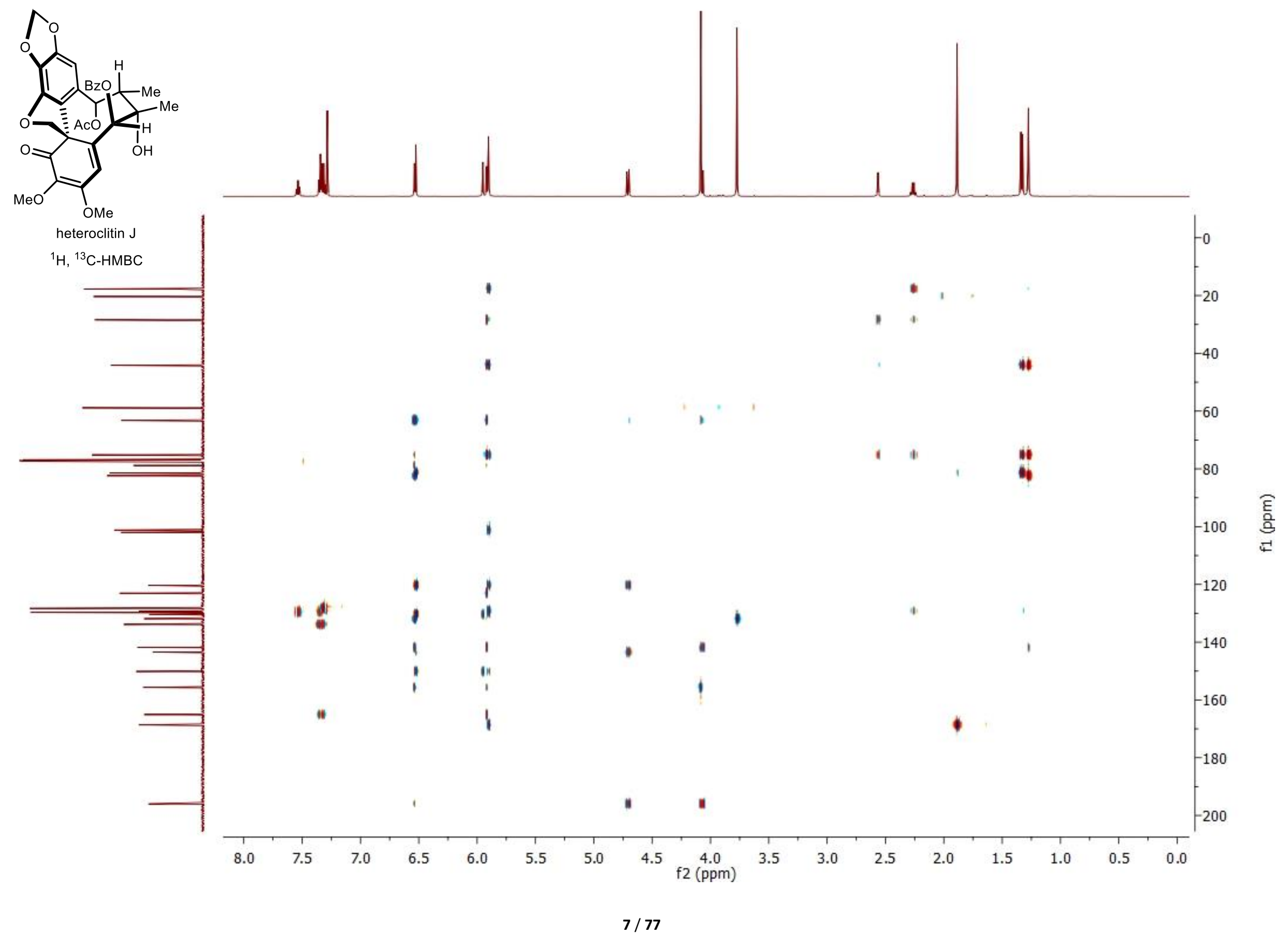


${ }^{1} \mathrm{H}-\mathrm{NMR}$ of compound 4 (kadsuphilin N)

\begin{tabular}{|c|c|c|c|c|c|}
\hline$\Phi$ & $\stackrel{\infty}{g}$ & 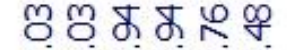 & Фొ & ถูก & 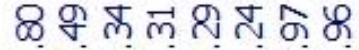 \\
\hline$\varphi$ & 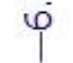 & ம் & ن & i & i \\
\hline
\end{tabular}

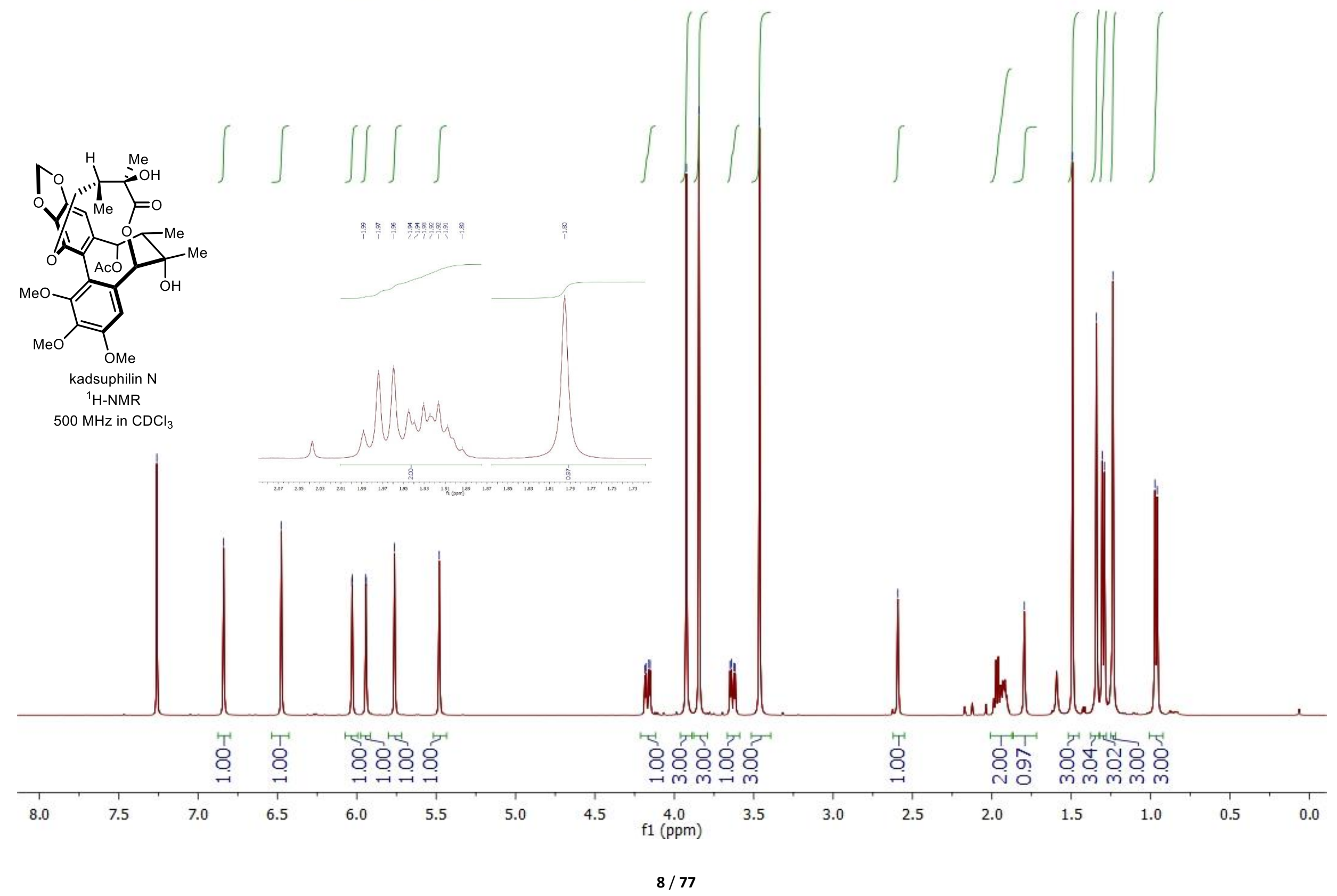




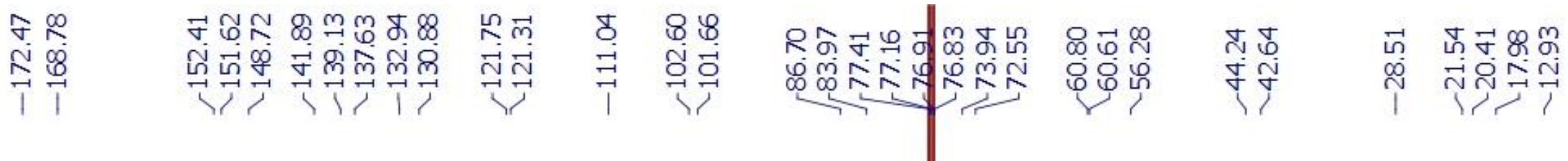

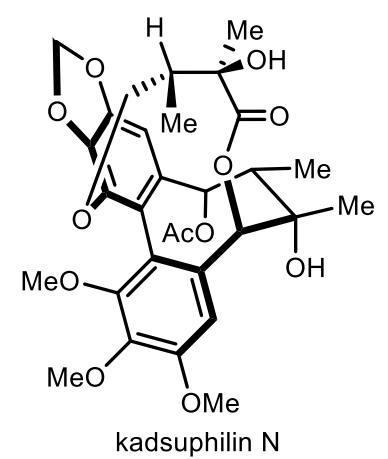

${ }^{13} \mathrm{C}-\mathrm{NMR}$

$126 \mathrm{MHz}$ in $\mathrm{CDCl}_{3}$ 
${ }^{1} \mathrm{H},{ }^{1} \mathrm{H}$-COSY of compound 4 (kadsuphilin $\mathrm{N}$ )

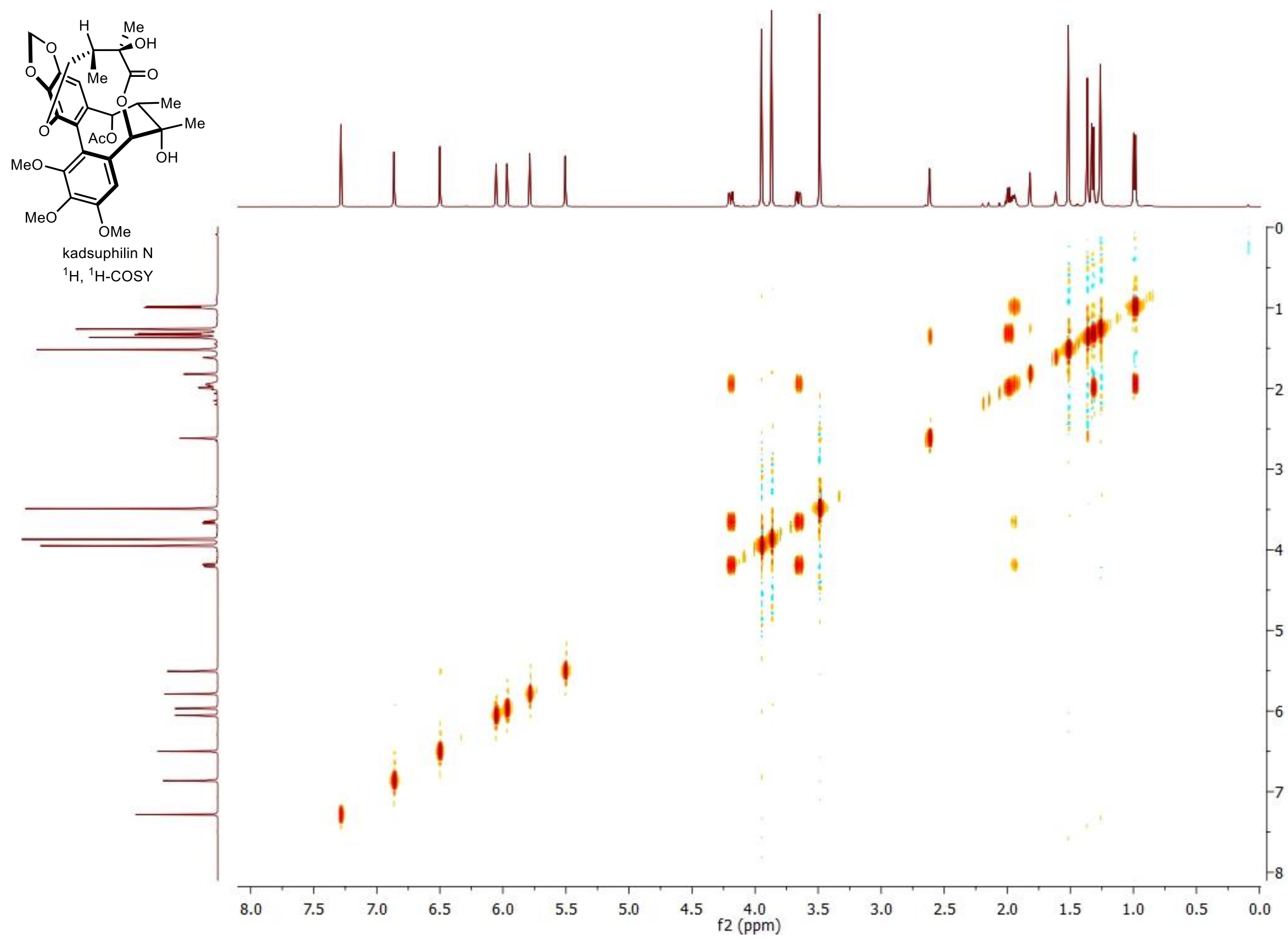


${ }^{1} \mathrm{H},{ }^{1} \mathrm{H}-\mathrm{NOESY}$ of compound 4 (kadsuphilin $\mathrm{N}$ )

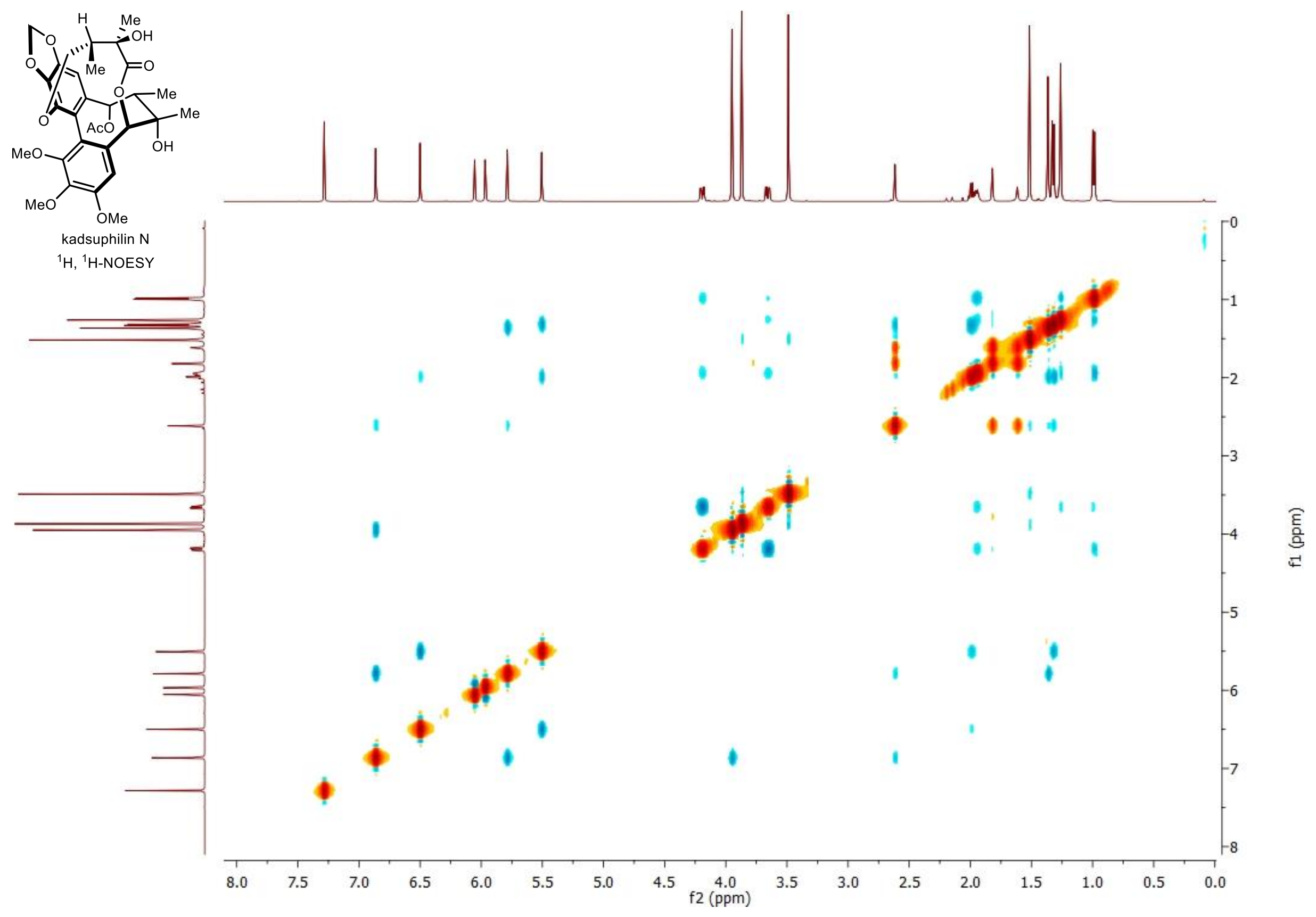

$11 / 77$ 
${ }^{1} \mathrm{H},{ }^{13} \mathrm{C}-\mathrm{HSQC}$ of compound 4 (kadsuphilin $\mathrm{N}$ )

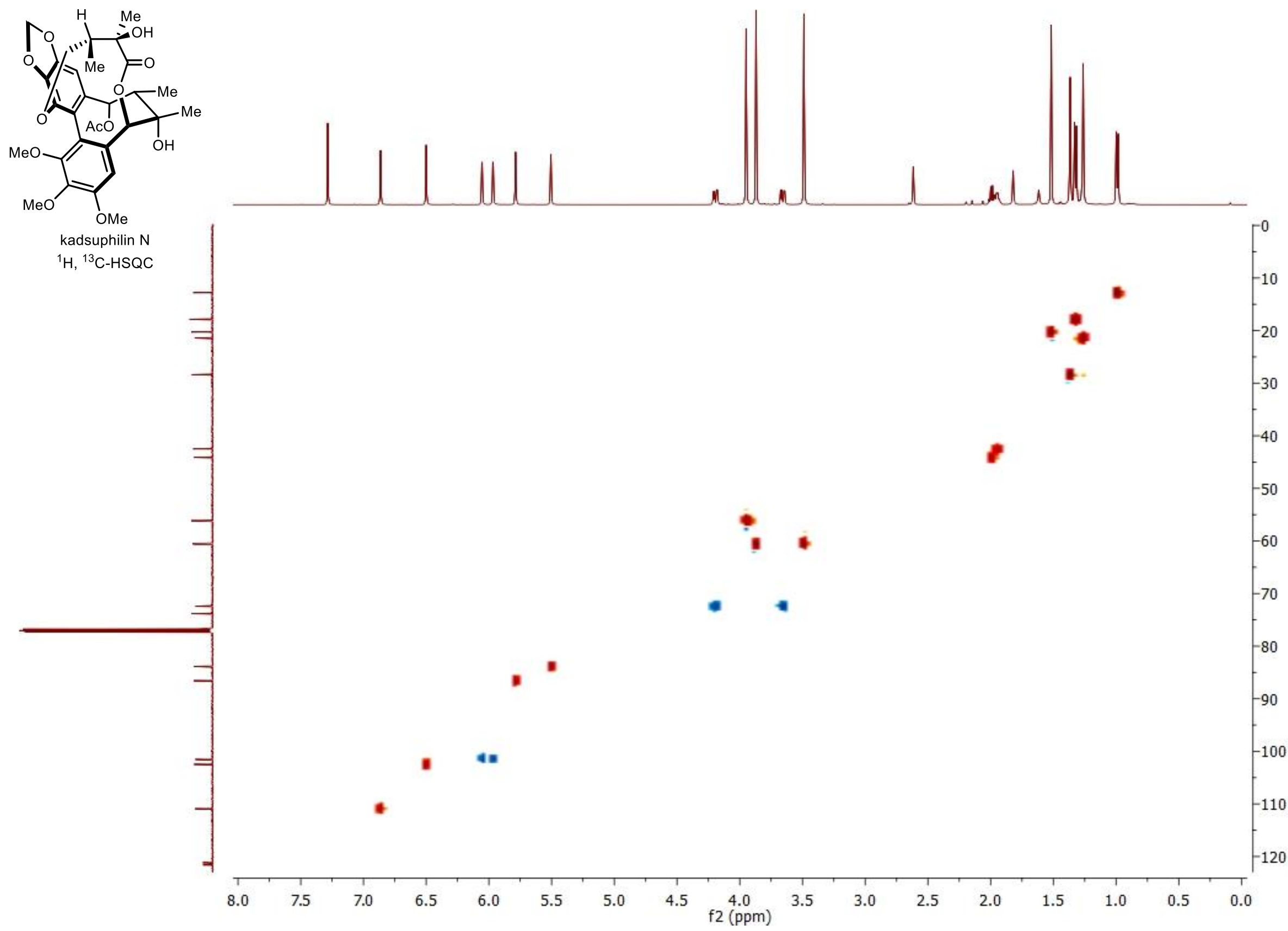


${ }^{1} \mathrm{H},{ }^{13} \mathrm{C}-\mathrm{HMBC}$ of compound 4 (kadsuphilin $\mathrm{N}$ )

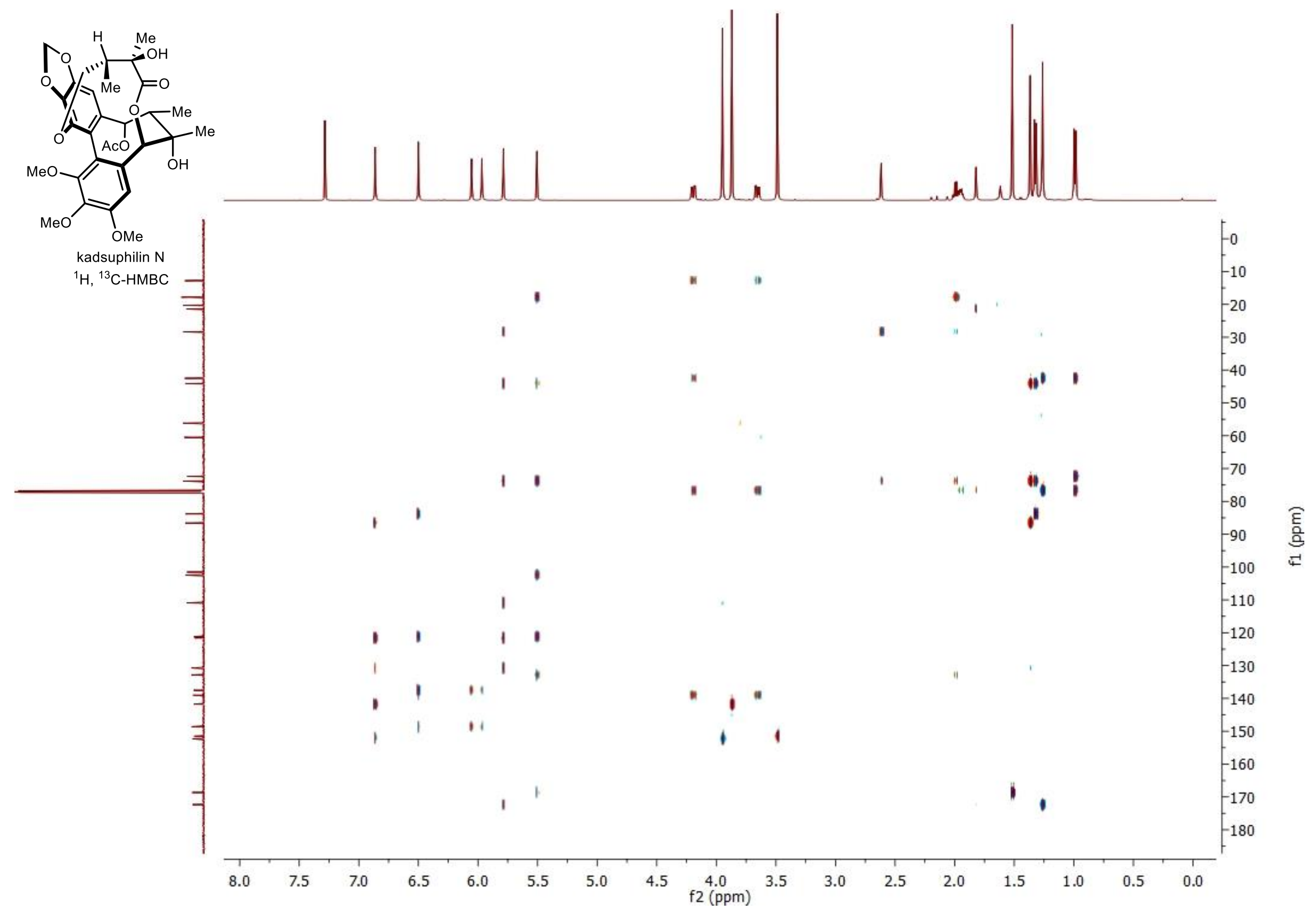

13 / 77 


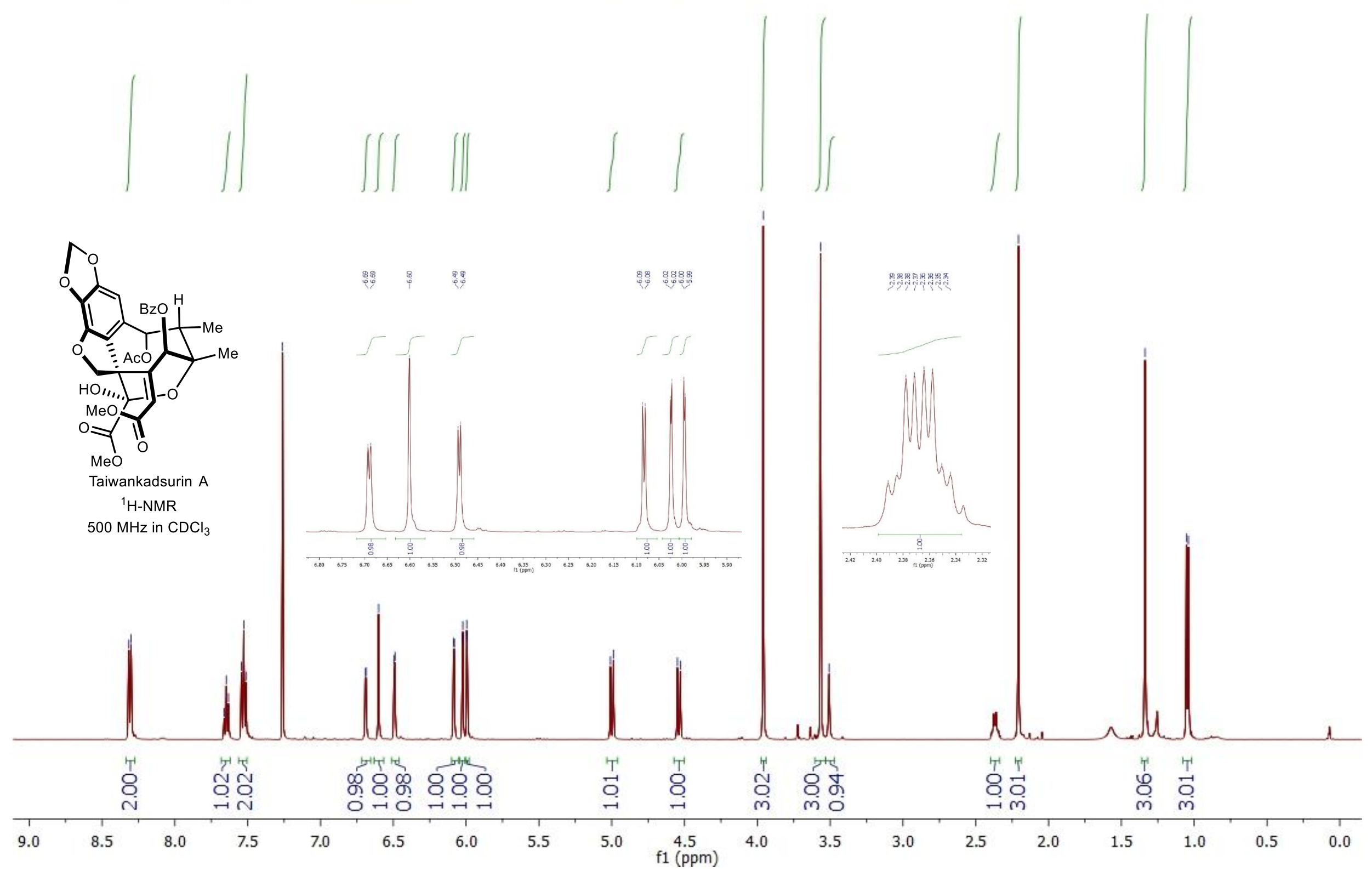


구음

-

gำ

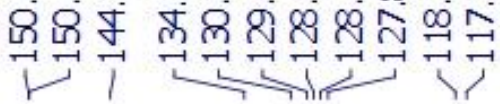

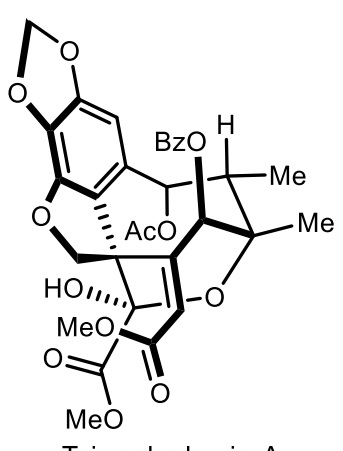

Taiwankadsurin A

$$
{ }^{13} \mathrm{C}-\mathrm{NMR}
$$

$126 \mathrm{MHz}$ in $\mathrm{CDCl}_{3}$

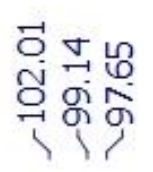

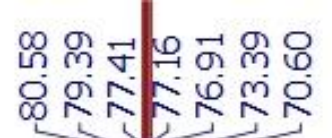

음 매요

กิ่

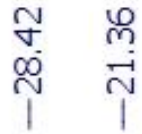

$\underset{\substack{\infty \\ \infty}}{\infty}$

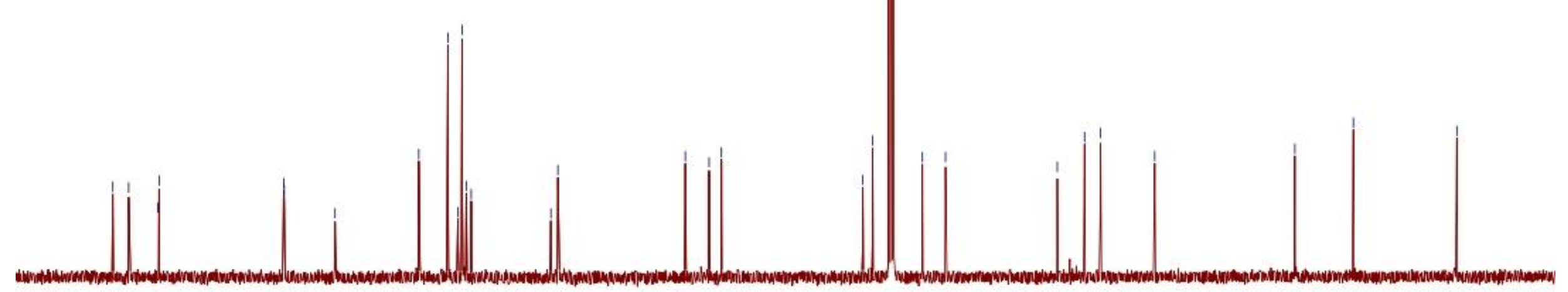


${ }^{1} \mathrm{H},{ }^{1} \mathrm{H}-\mathrm{COSY}$ of compound $5 \mathrm{a}$ (taiwankadsurin $\mathrm{A}$ )

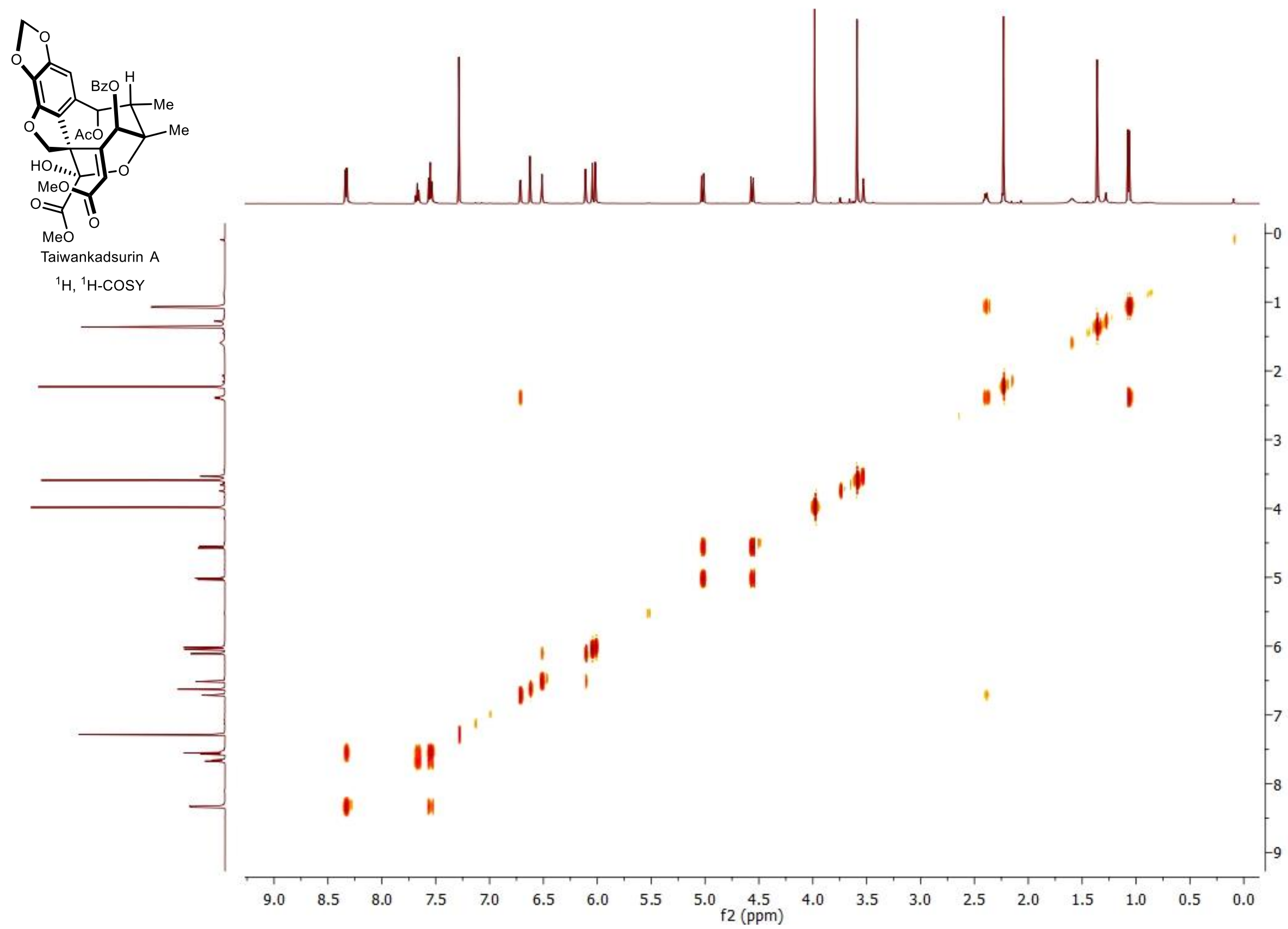


${ }^{1} \mathrm{H},{ }^{1} \mathrm{H}-\mathrm{NOESY}$ of compound $5 \mathrm{a}$ (taiwankadsurin $\mathrm{A}$ )

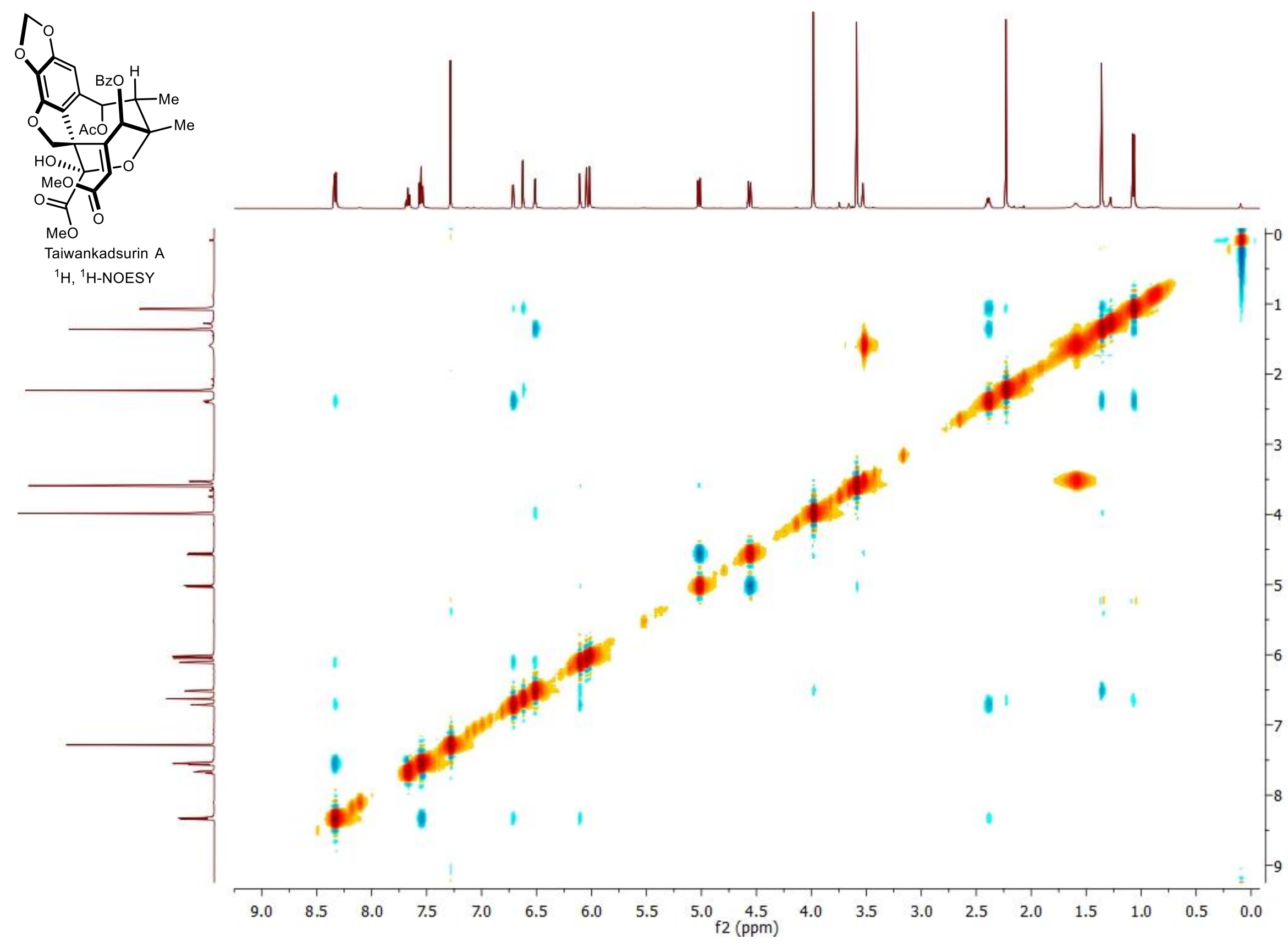


${ }^{1} \mathrm{H},{ }^{13} \mathrm{C}-\mathrm{HSQC}$ of compound $5 \mathrm{a}$ (taiwankadsurin $\mathrm{A}$ )
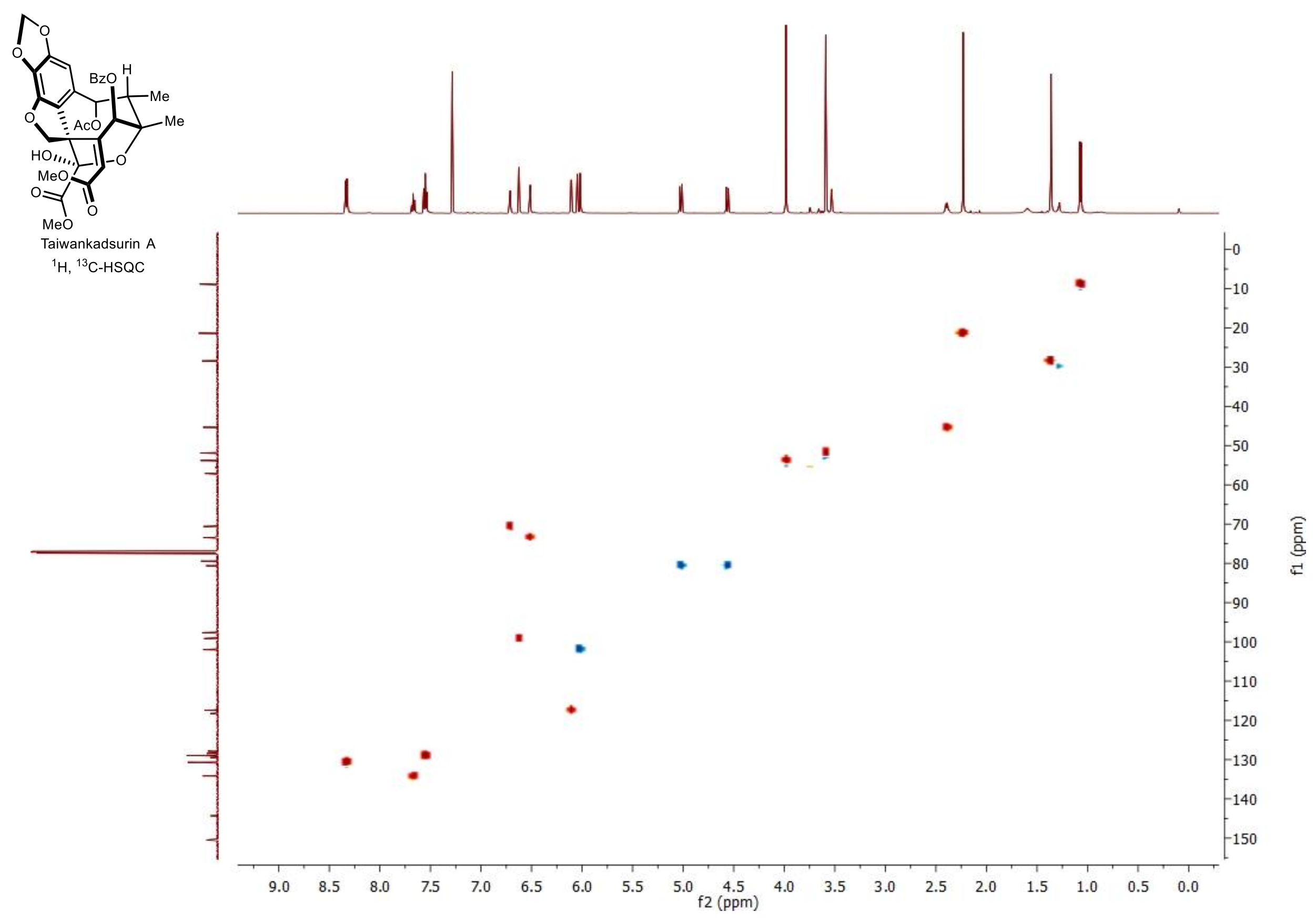

18 / 77 
${ }^{1} \mathrm{H},{ }^{13} \mathrm{C}-\mathrm{HMBC}$ of compound $5 \mathrm{a}$ (taiwankadsurin $\mathrm{A}$ )

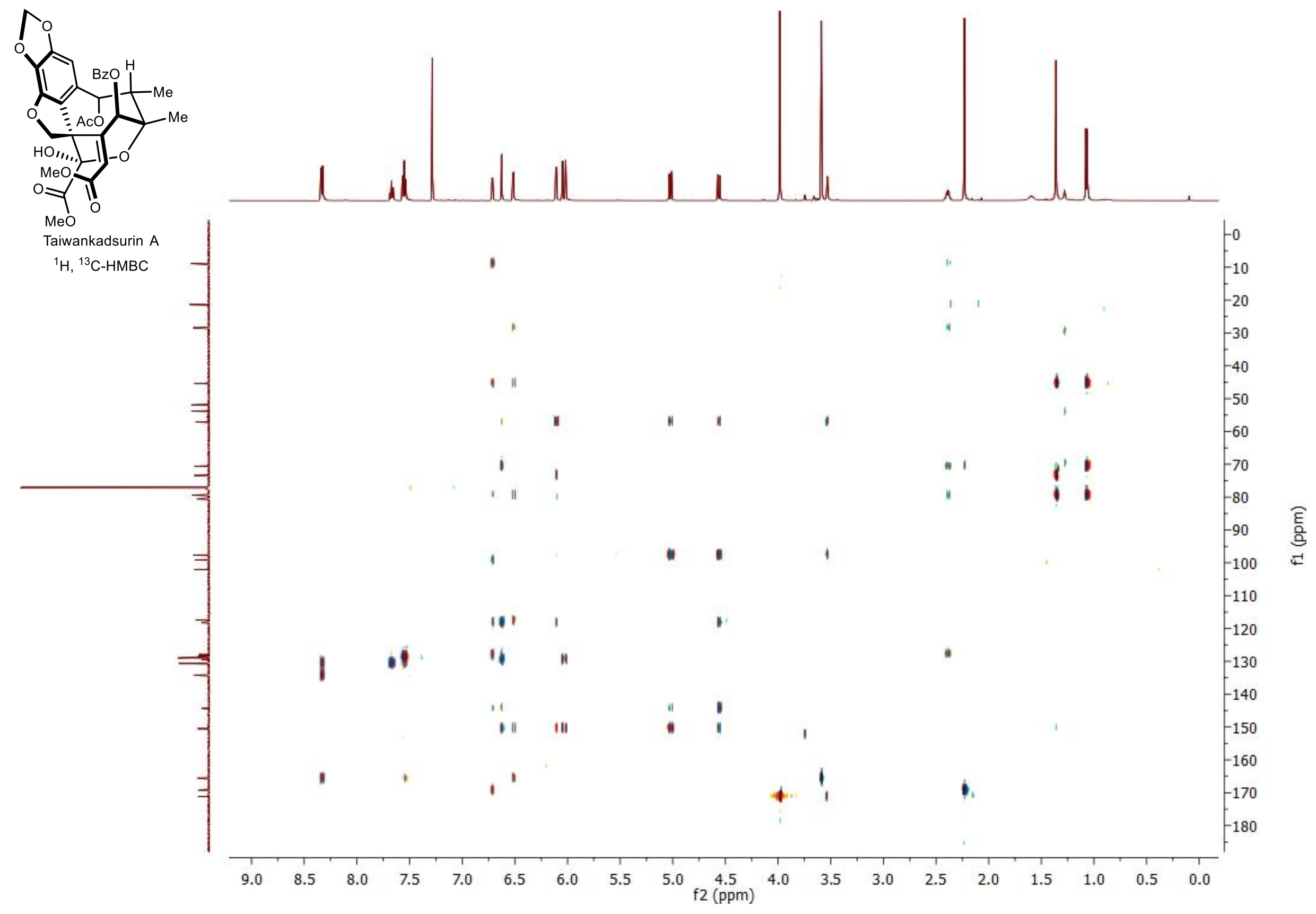

19 / 77 
ભ $\infty \infty$

\section{न 8 웅

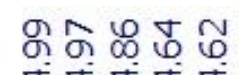

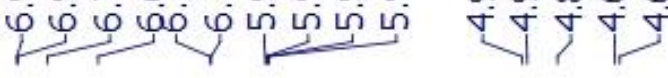

$\overrightarrow{0}$

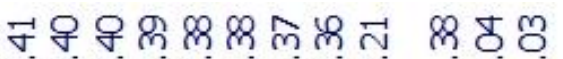
का

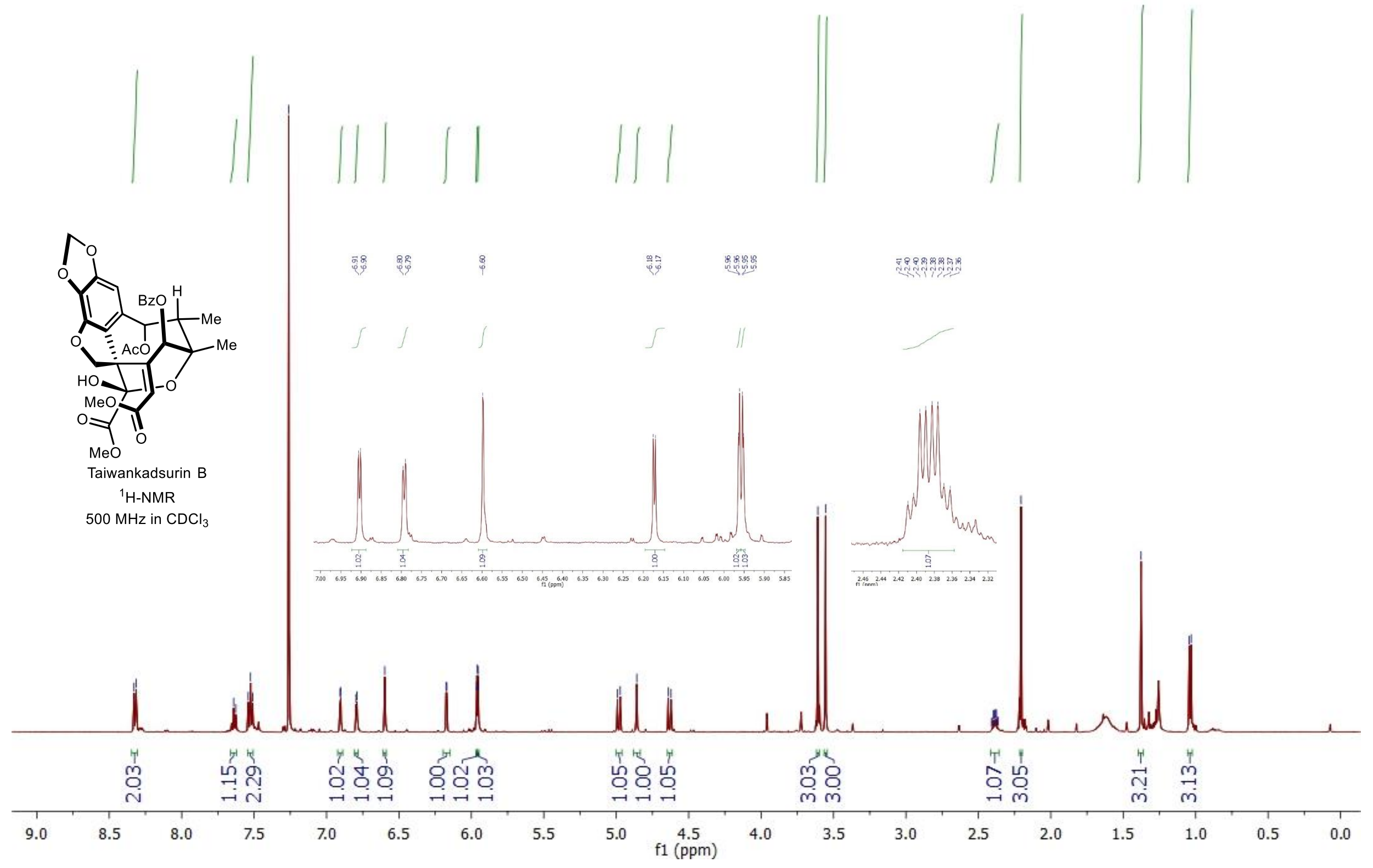


${ }^{13} \mathrm{C}-\mathrm{NMR}$ of compound $\mathbf{5 b}$ (taiwankadsurin B)

$\therefore$ 边

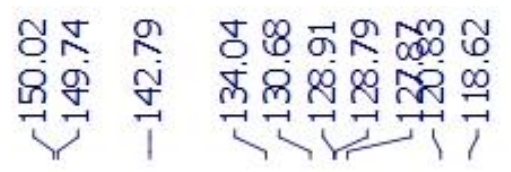

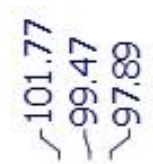

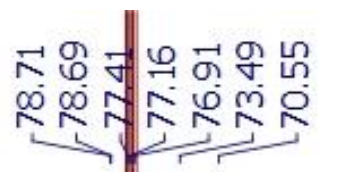

œ

舟它的毕

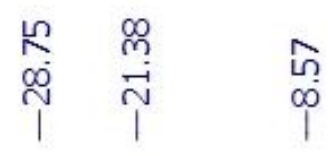

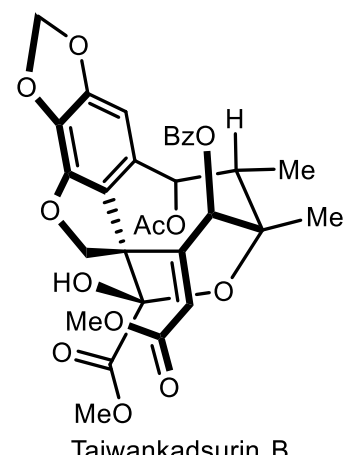

Taiwankadsurin B

${ }^{13} \mathrm{C}-\mathrm{NMR}$

$126 \mathrm{MHz}$ in $\mathrm{CDCl}_{3}$

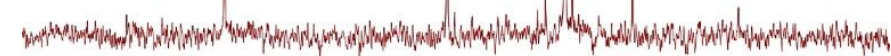
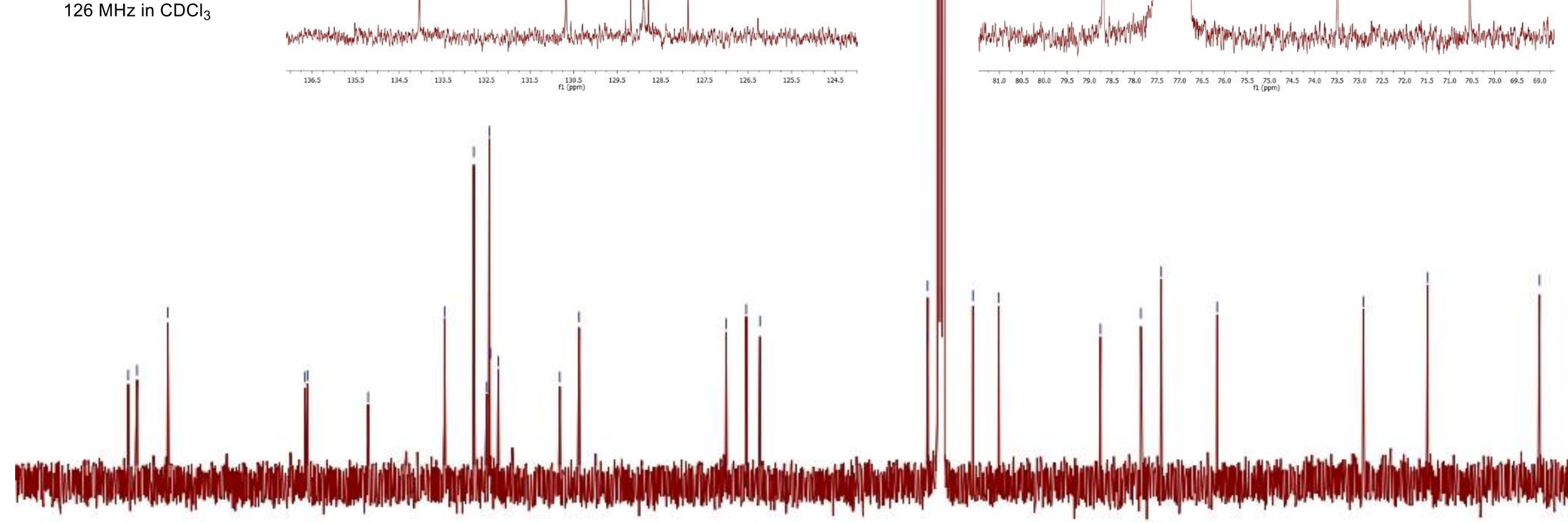

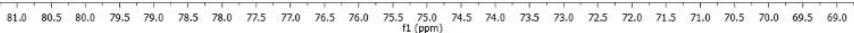


${ }^{1} \mathrm{H},{ }^{1} \mathrm{H}-\mathrm{COSY}$ of compound $\mathbf{5 b}$ (taiwankadsurin $\mathrm{B}$ )

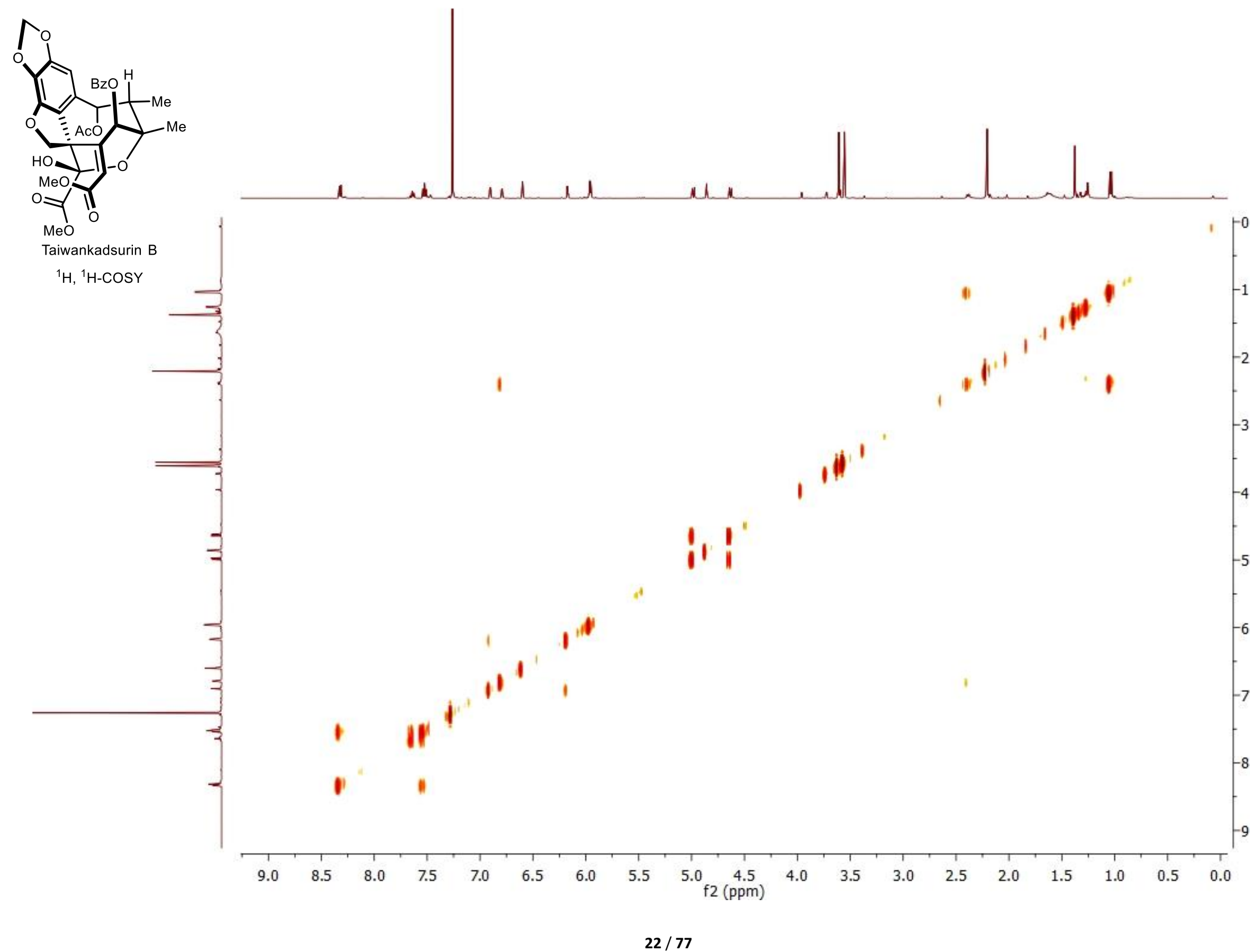


${ }^{1} \mathrm{H},{ }^{1} \mathrm{H}-\mathrm{NOESY}$ of compound $\mathbf{5 b}$ (taiwankadsurin $\mathrm{B}$ )

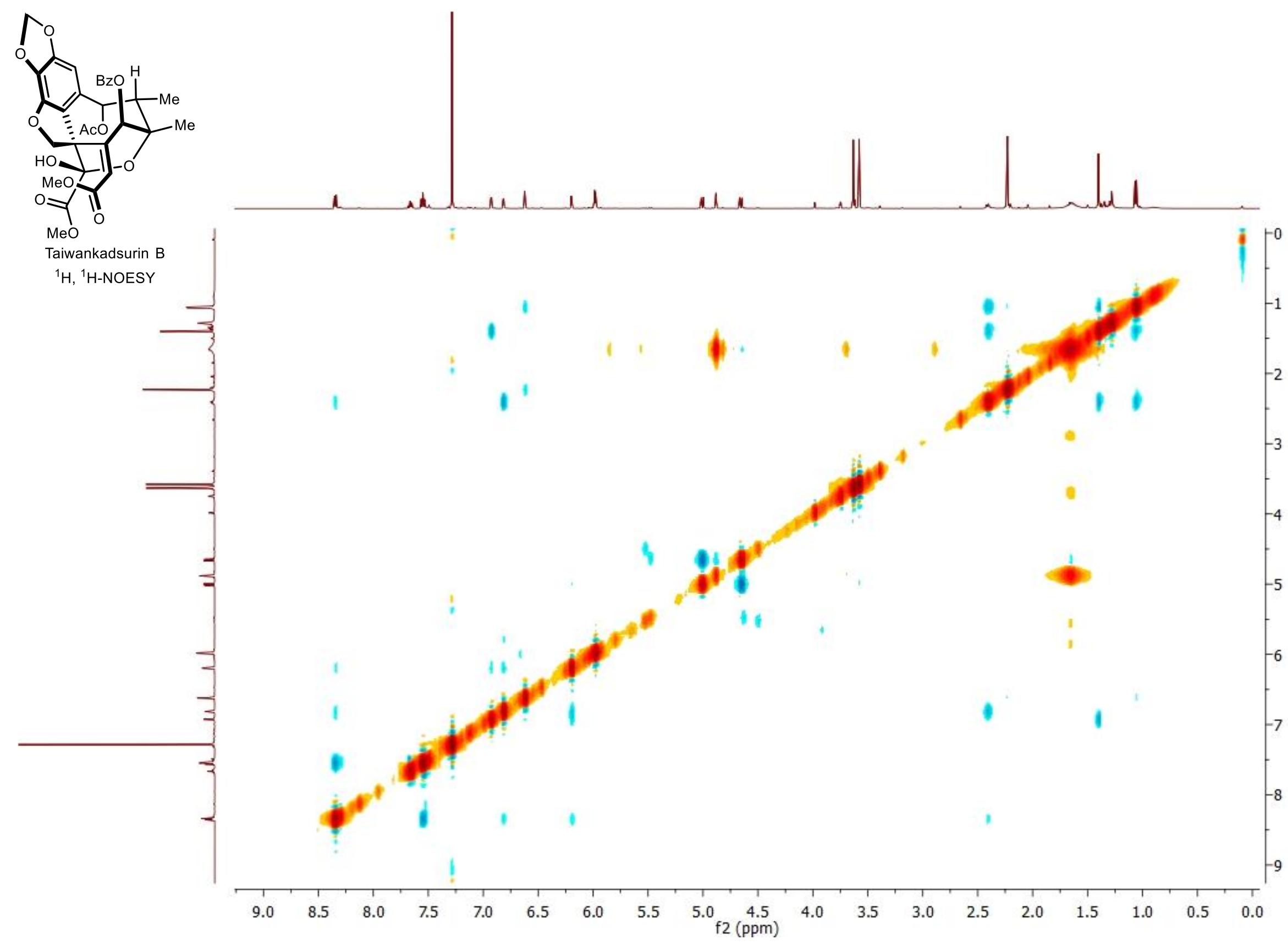


${ }^{1} \mathrm{H},{ }^{13} \mathrm{C}-\mathrm{HSQC}$ of compound $\mathbf{5 b}$ (taiwankadsurin $\mathrm{B}$ )

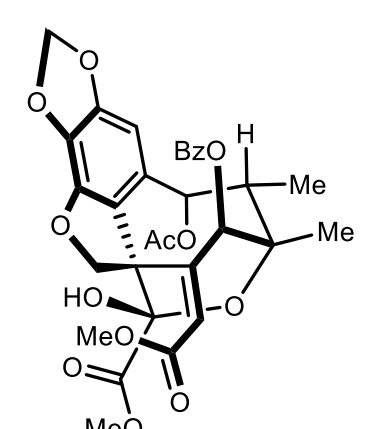

$\mathrm{MeO}$

Taiwankadsurin $\mathrm{B}$
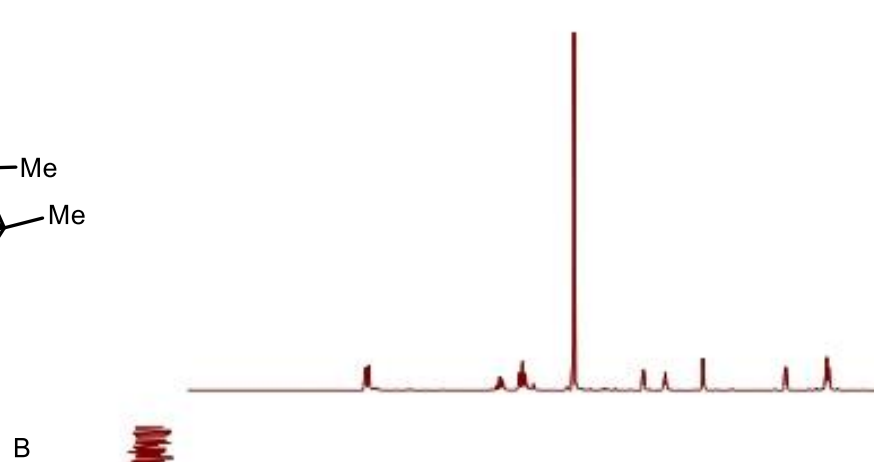

${ }^{1} \mathrm{H},{ }^{13} \mathrm{C}-\mathrm{HSQC}$ 
${ }^{1} \mathrm{H},{ }^{13} \mathrm{C}-\mathrm{HMBC}$ of compound $\mathbf{5 b}$ (taiwankadsurin $\mathrm{B}$ )
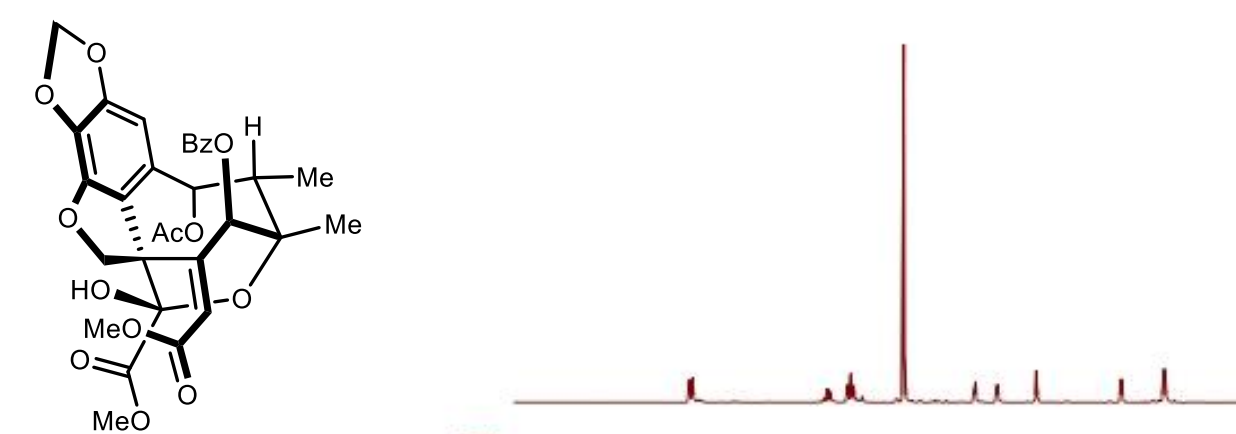

11

Taiwankadsurin $\mathrm{B}$

${ }^{1} \mathrm{H},{ }^{13} \mathrm{C}-\mathrm{HMBC}$
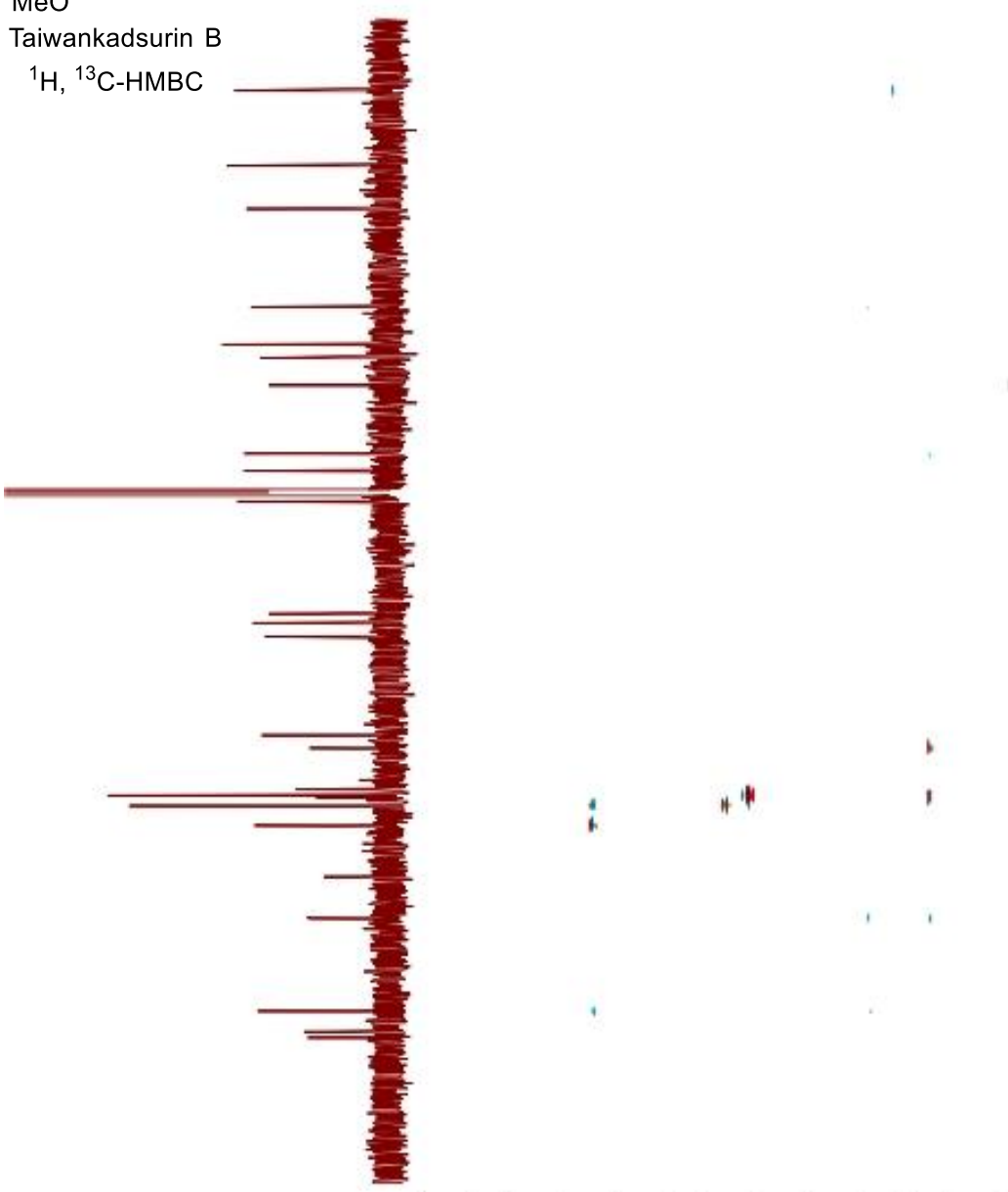

$$
9.0
$$

5.0
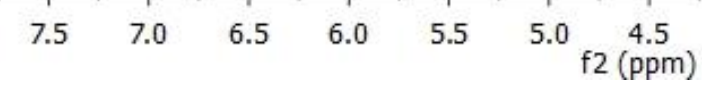

$25 / 77$ 


\section{${ }^{1} \mathrm{H}-\mathrm{NMR}$ of compound 22}

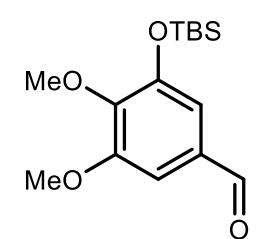

${ }^{1} \mathrm{H}-\mathrm{NMR}$

$500 \mathrm{MHz}$ in $\mathrm{CDCl}_{3}$

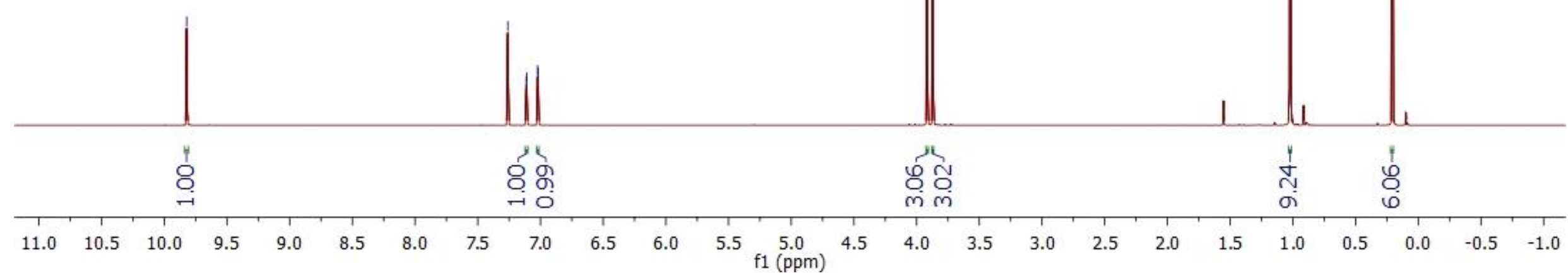


${ }^{13} \mathrm{C}-\mathrm{NMR}$ of compound 22

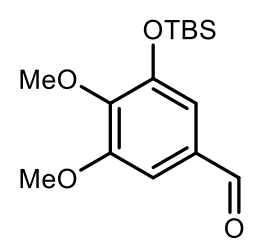

${ }^{13} \mathrm{C}-\mathrm{NMR}$

$126 \mathrm{MHz}$ in $\mathrm{CDCl}_{3}$

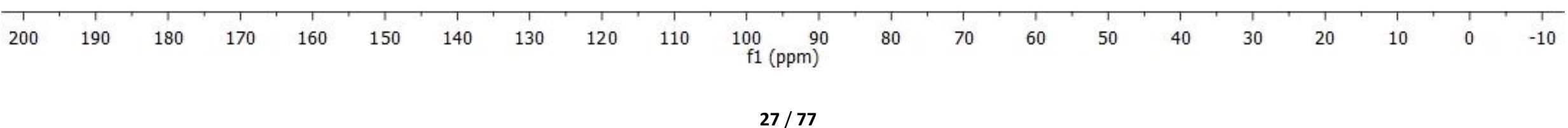


${ }^{1} \mathrm{H}-\mathrm{NMR}$ of compound SI-1

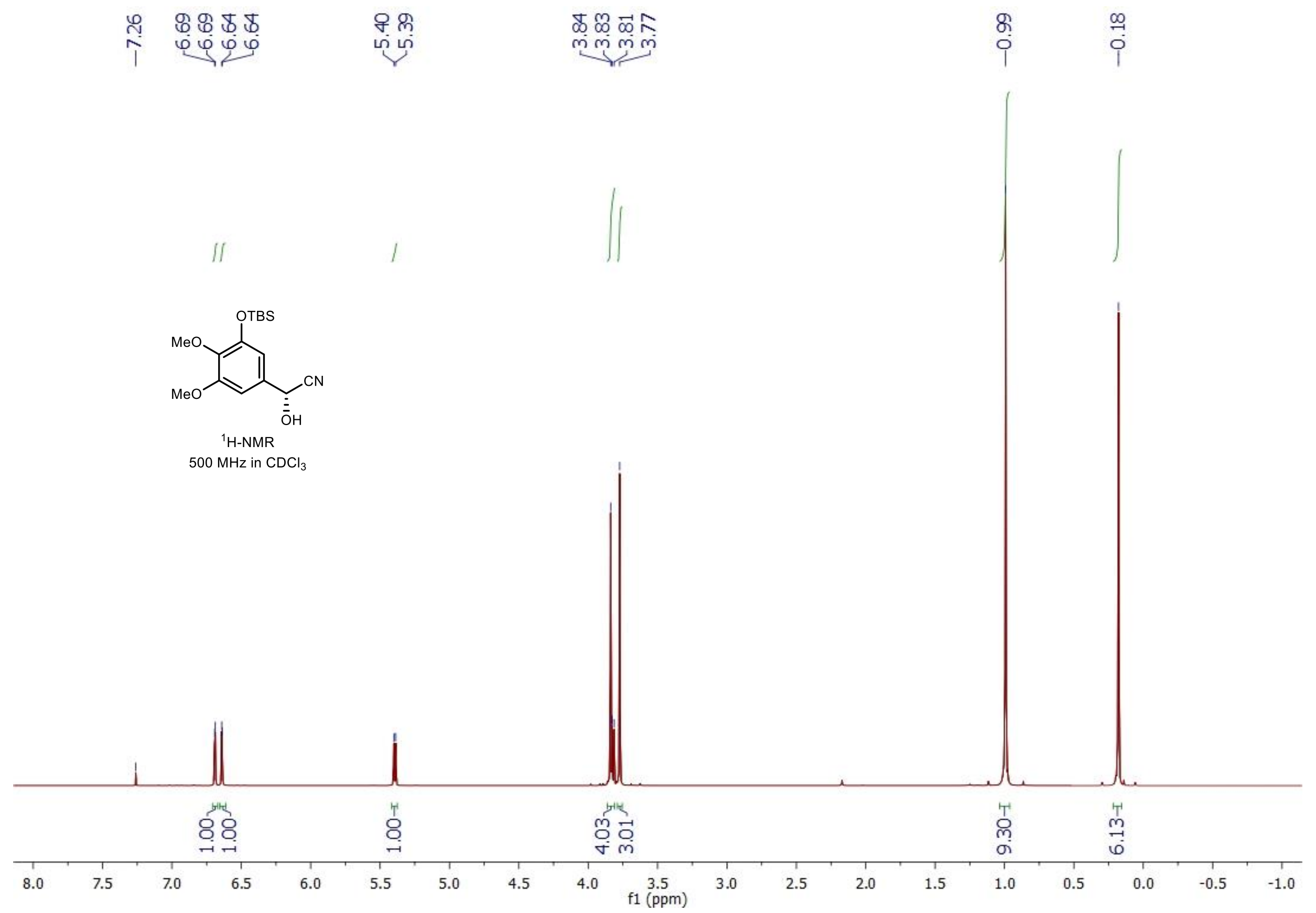




\section{${ }^{13} \mathrm{C}-\mathrm{NMR}$ of compound SI-1}

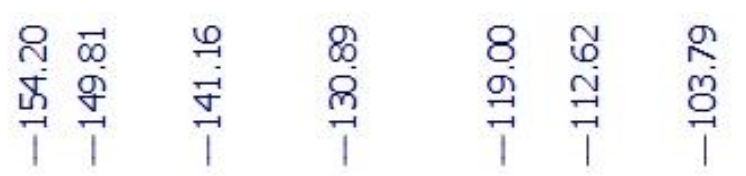

चु

लिए m

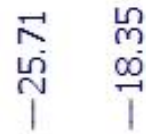

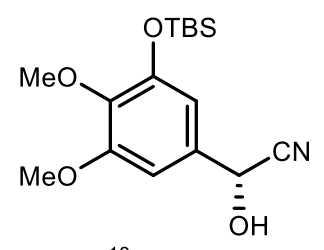

${ }^{13} \mathrm{C}-\mathrm{NMR}$

$126 \mathrm{MHz}$ in $\mathrm{CDCl}_{3}$

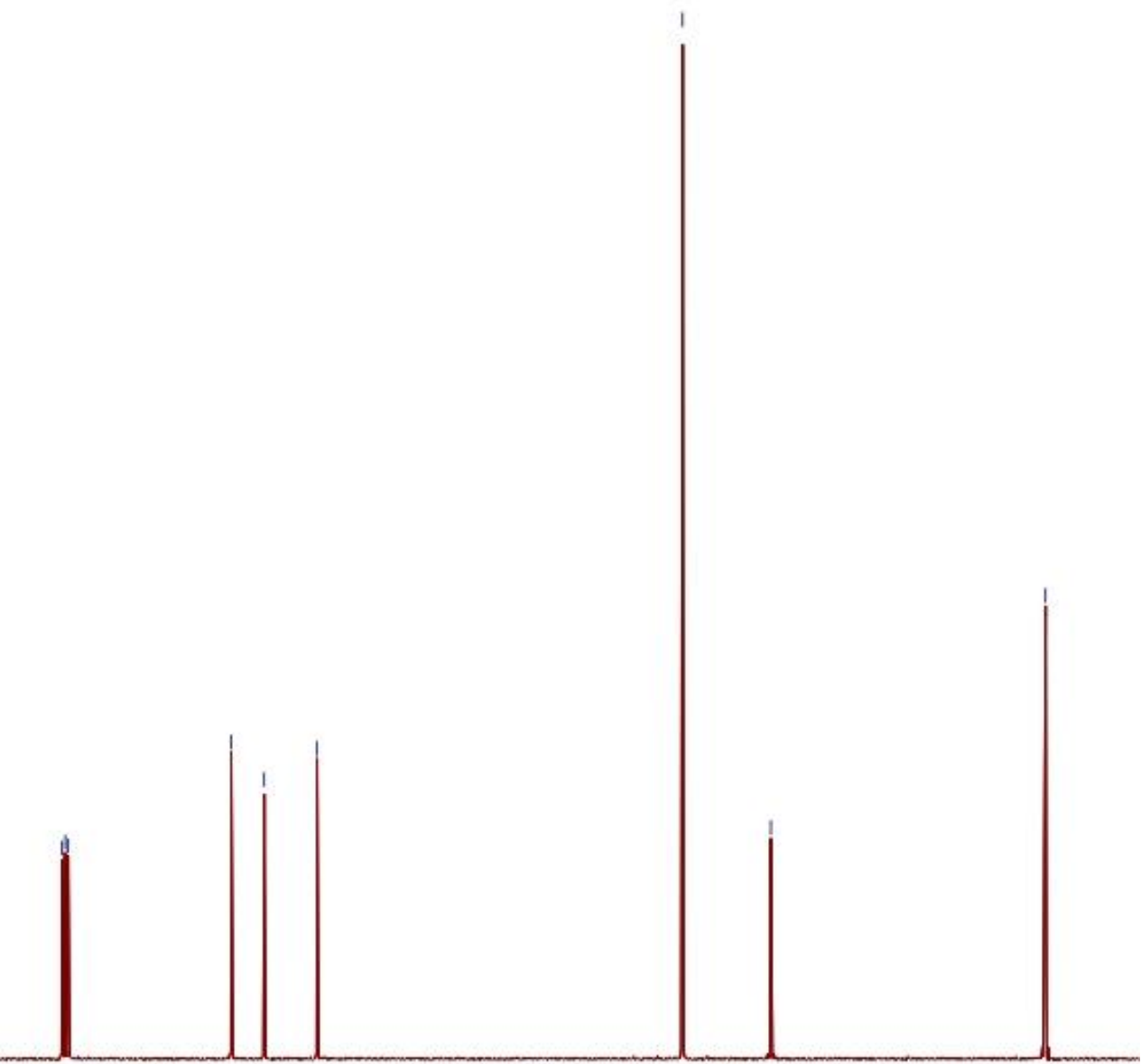

$$
17
$$


ำ

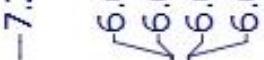

\section{t}

ऍ

${ }^{1} \mathrm{H}-\mathrm{NMR}$

$500 \mathrm{MHz}$ in $\mathrm{CDCl}_{3}$

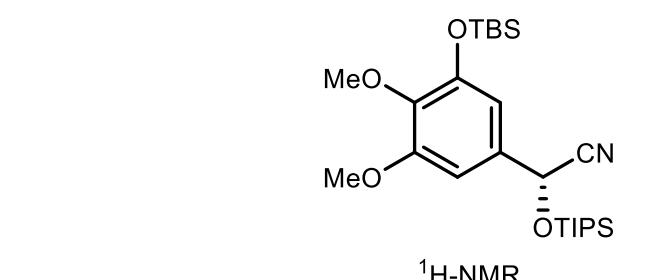

॥

80

m?
구을요

$\mid$

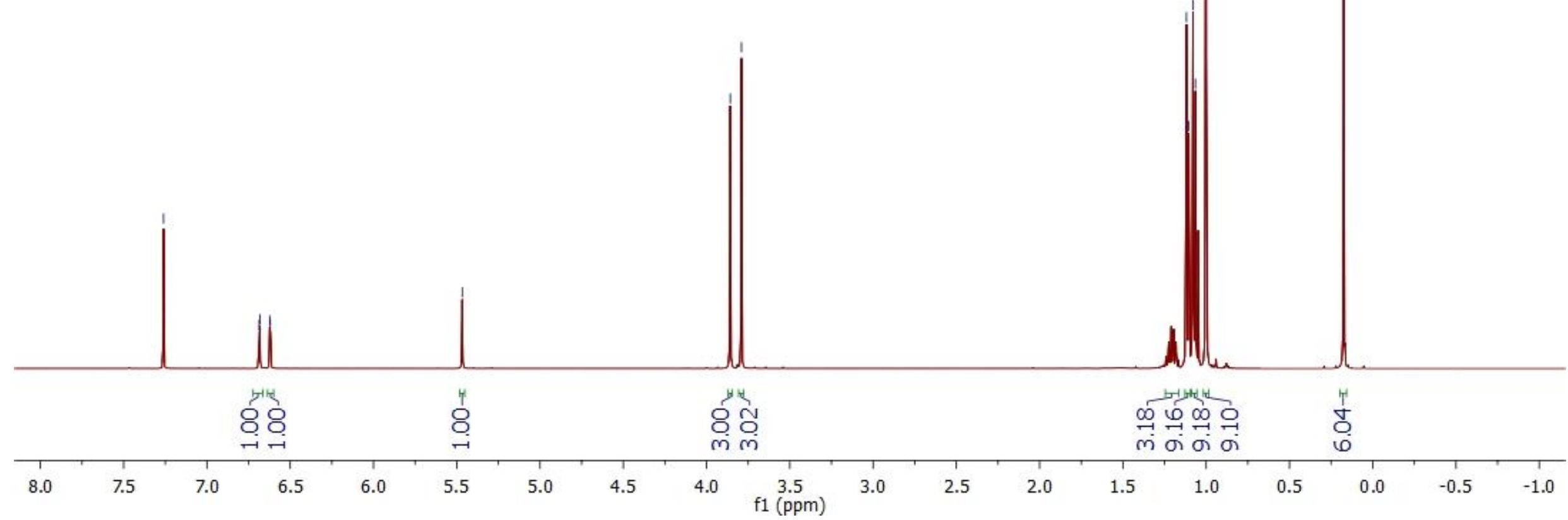

$30 / 77$ 
${ }^{13} \mathrm{C}-\mathrm{NMR}$ of compound SI-2

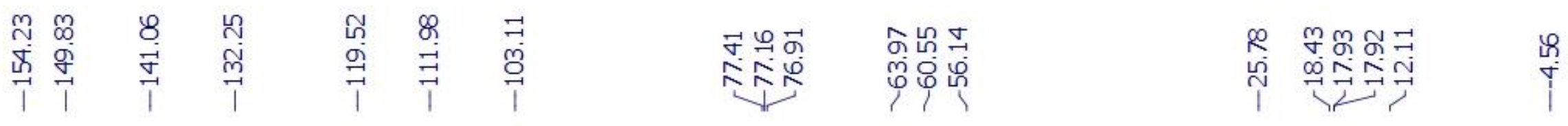

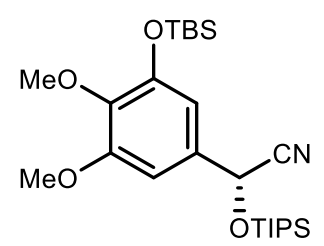

${ }^{13} \mathrm{C}-\mathrm{NMR}$

$126 \mathrm{MHz}$ in $\mathrm{CDCl}_{3}$

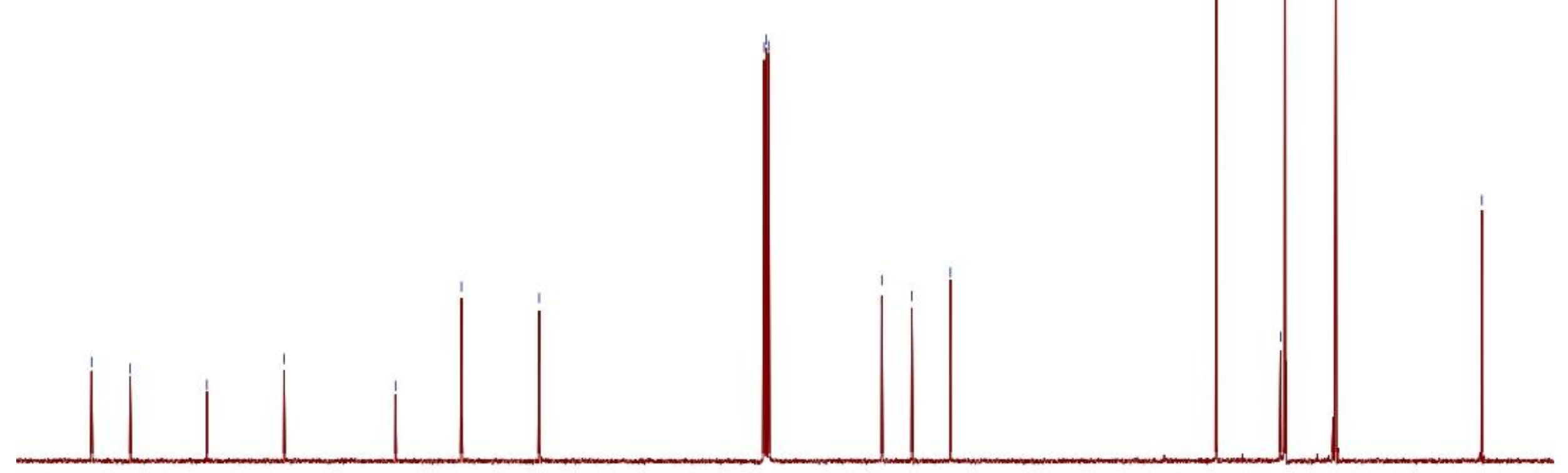




\section{${ }^{1} \mathrm{H}-\mathrm{NMR}$ of compound 24}

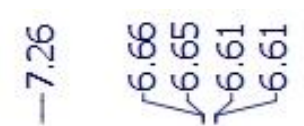

एํา

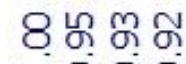

궁잉

는 는

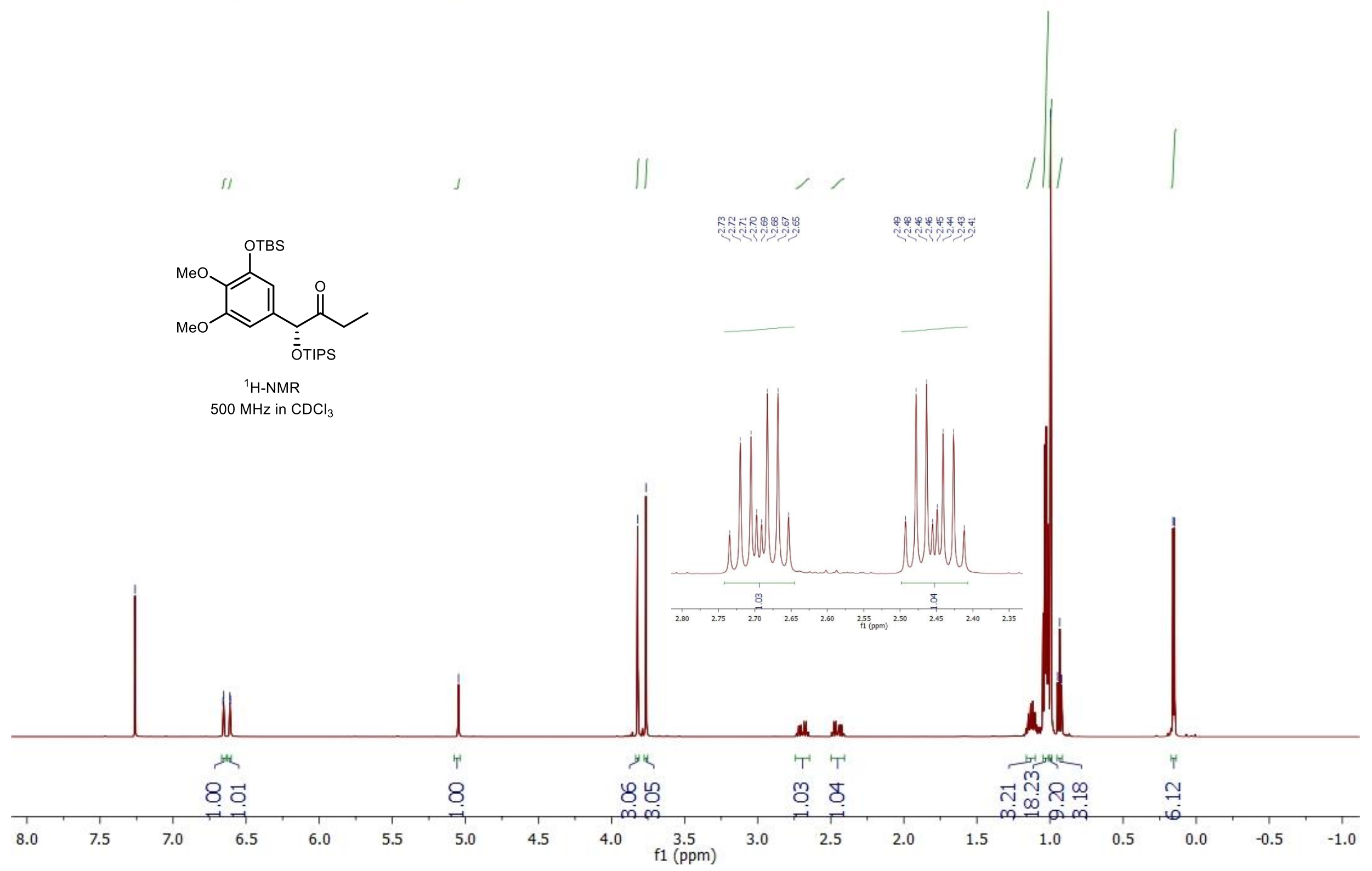


${ }^{13} \mathrm{C}-\mathrm{NMR}$ of compound 24

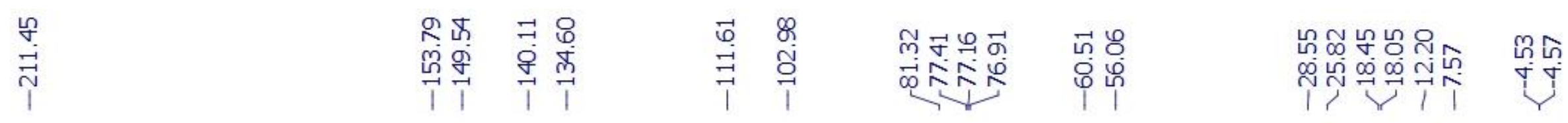

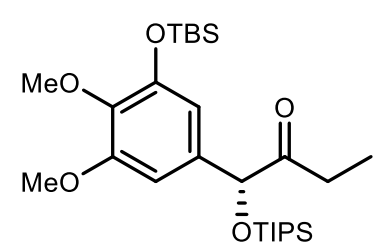

${ }^{13} \mathrm{C}-\mathrm{NMR}$

$126 \mathrm{MHz}$ in $\mathrm{CDCl}_{3}$

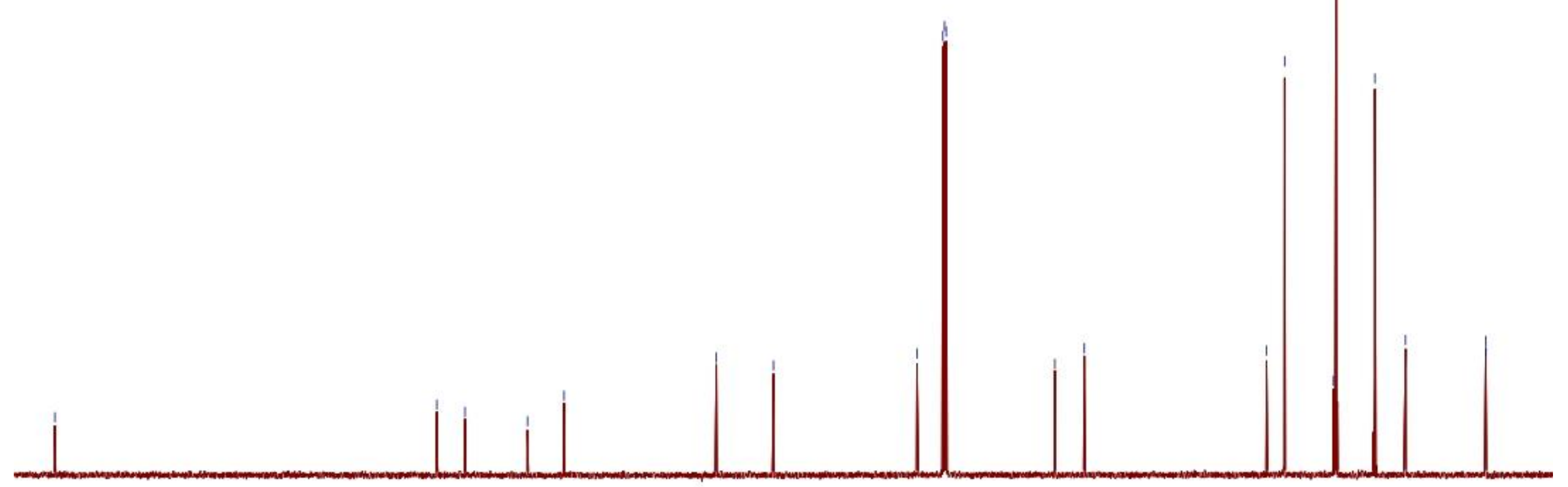

210

$200 \quad 190 \quad 180 \quad 170 \quad 160$

140

130

$110 \stackrel{100}{\mathrm{f} 1(\mathrm{ppm})}$
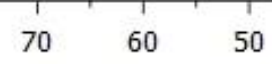

40

30 


\section{${ }^{1} \mathrm{H}-\mathrm{NMR}$ of compound 26}

$\stackrel{\text { : }}{\stackrel{ }{1}}$

to

i

$\begin{array}{ll}0 & m \\ 0 & m \\ 0 & 1\end{array}$

$\overrightarrow{0}$

।

in

i
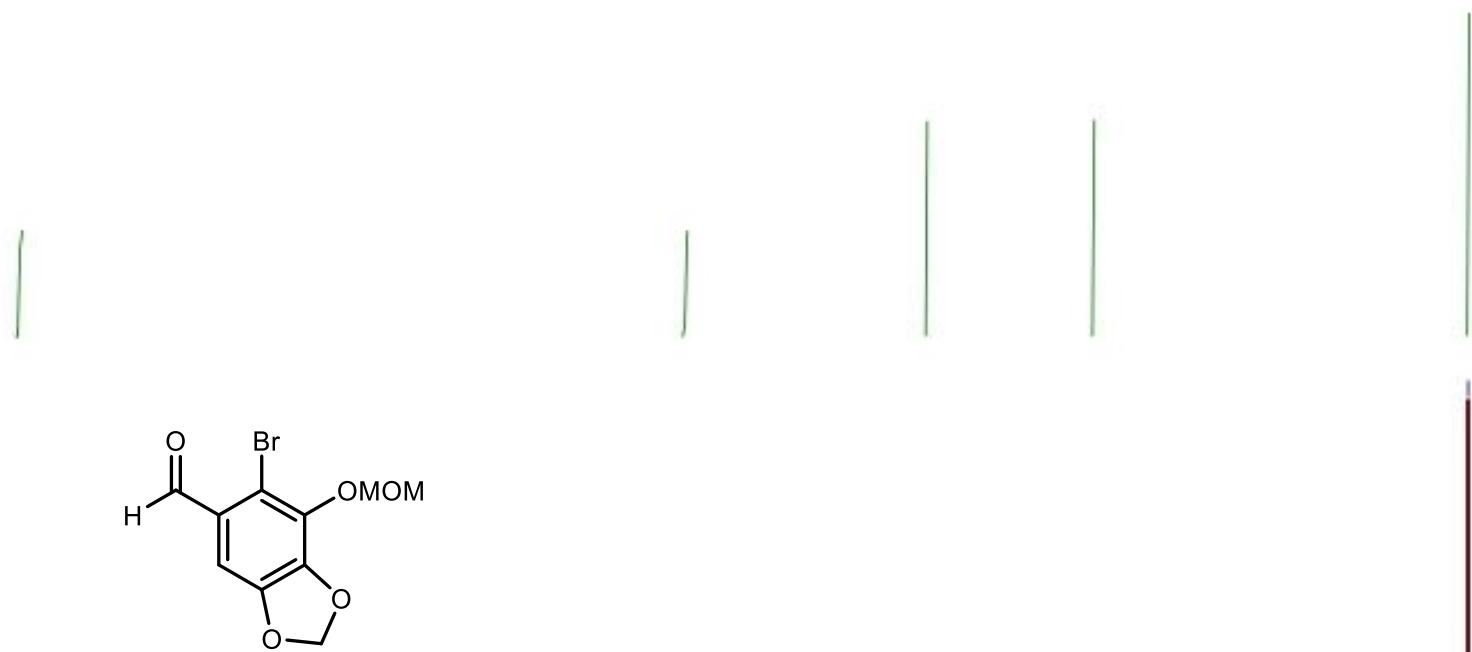

${ }^{1} \mathrm{H}-\mathrm{NMR}$

$500 \mathrm{MHz}$ in $\mathrm{CDCl}_{3}$

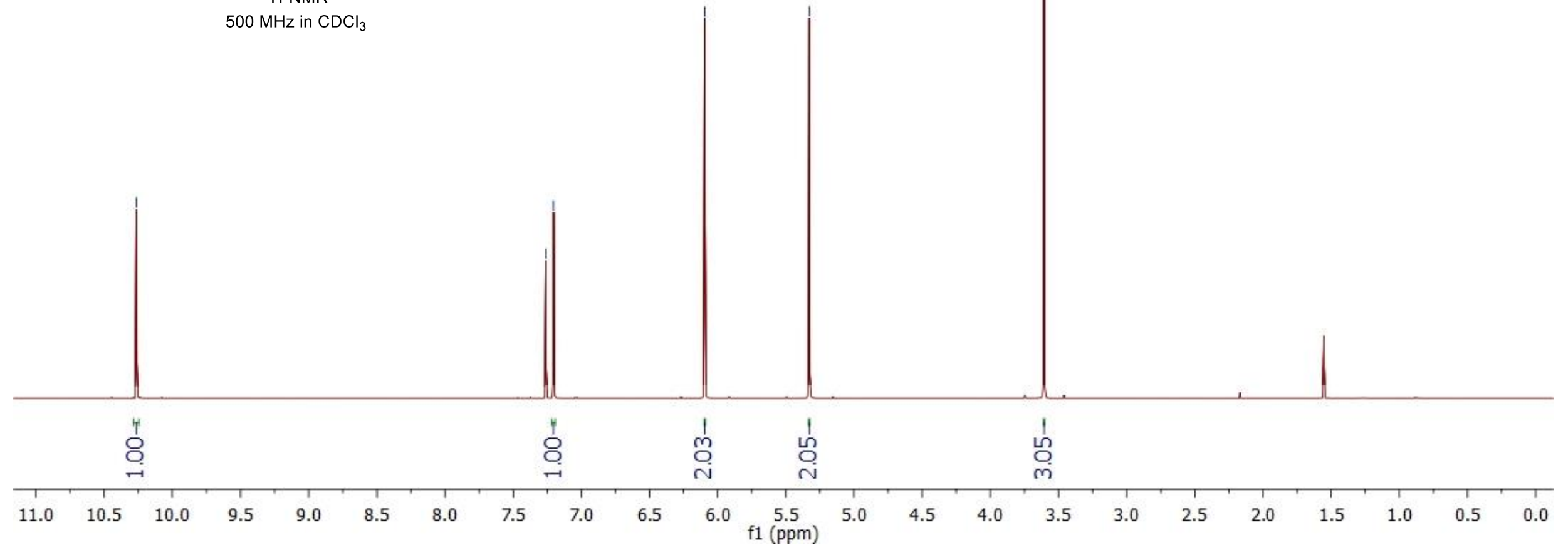

34 / 77 
${ }^{13} \mathrm{C}-\mathrm{NMR}$ of compound 26

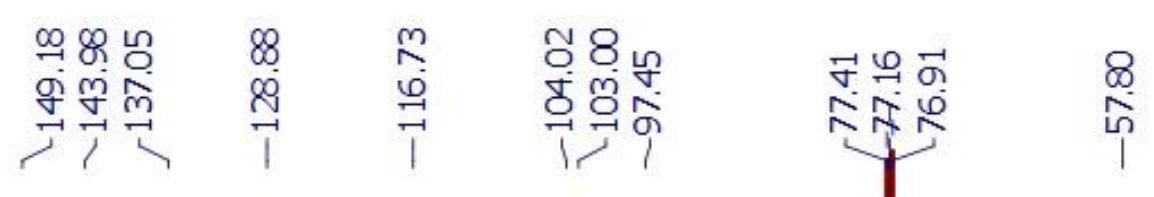

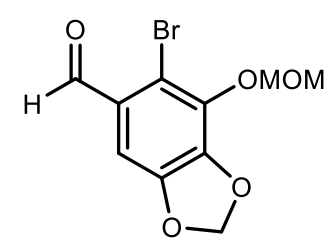

${ }^{13} \mathrm{C}-\mathrm{NMR}$

$126 \mathrm{MHz}$ in $\mathrm{CDCl}_{3}$

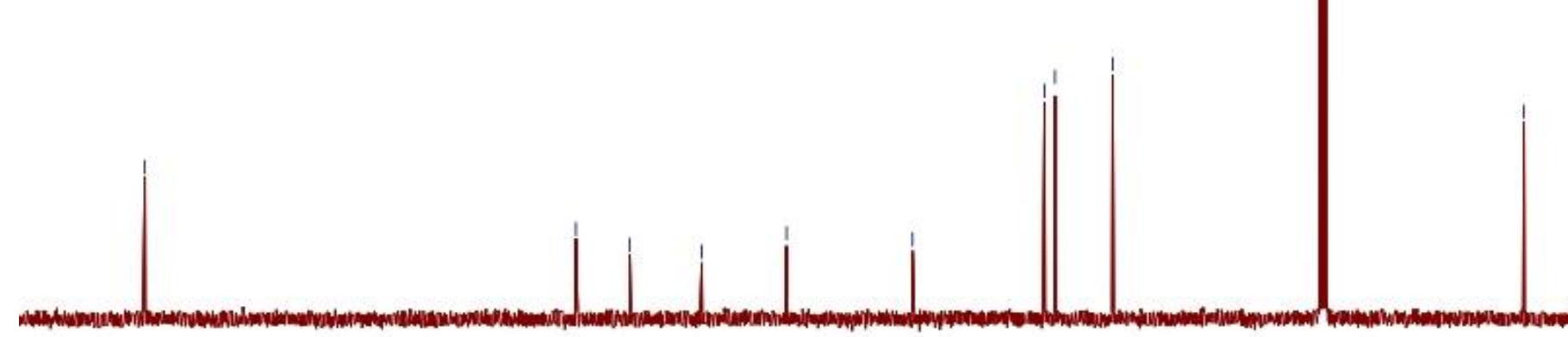




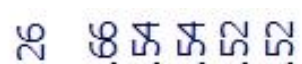

i

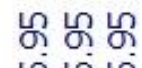

ก๊

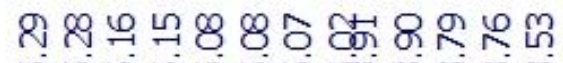

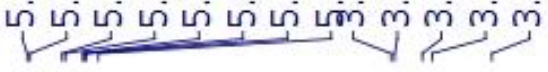

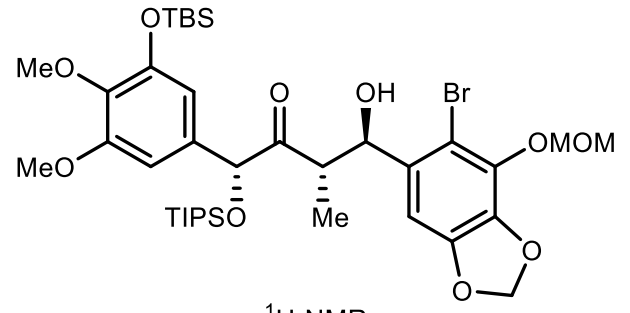

${ }^{1} \mathrm{H}-\mathrm{NMR}$

$500 \mathrm{MHz}$ in $\mathrm{CDCl}_{3}$

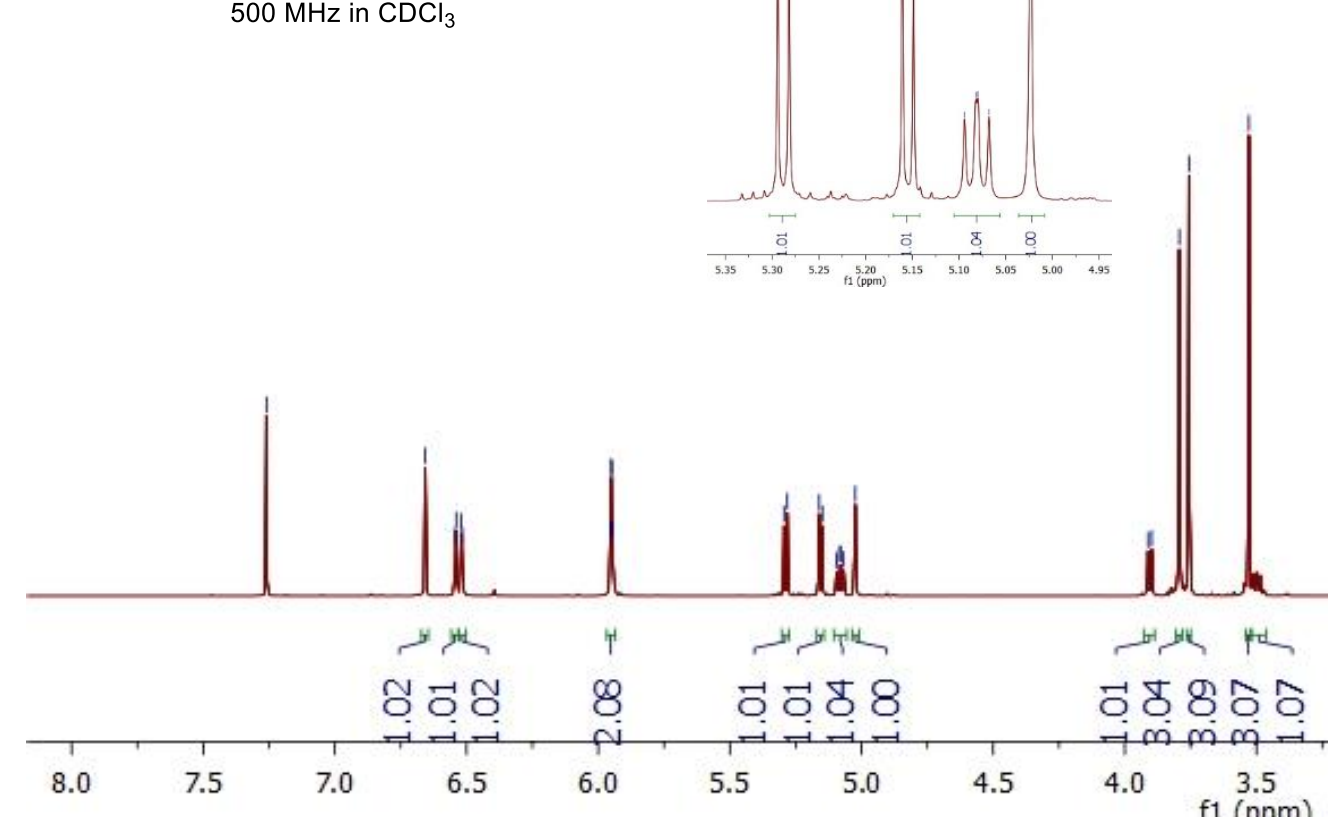

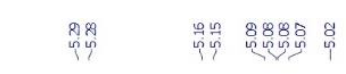

, II I,

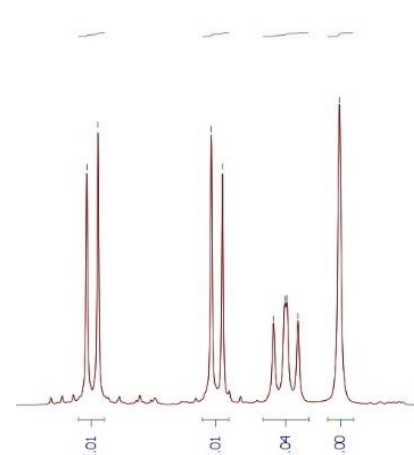

4.0 3.5
f1 $(\mathrm{ppm})$

苗西

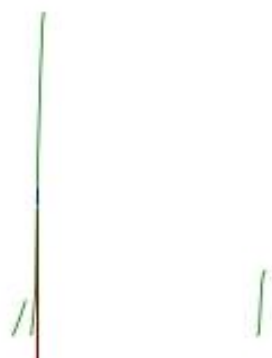

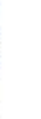

$36 / 77$ 
${ }^{13} \mathrm{C}-\mathrm{NMR}$ of compound 27

華

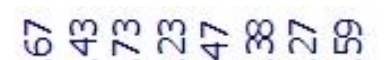

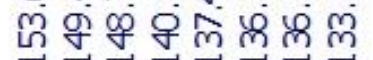

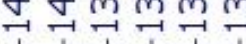

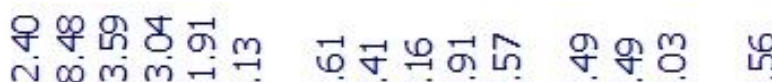

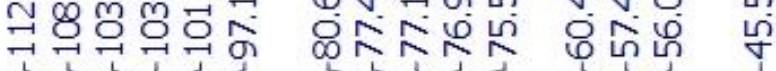

र $\overrightarrow{7} 88 \%$ no 0 ณ i

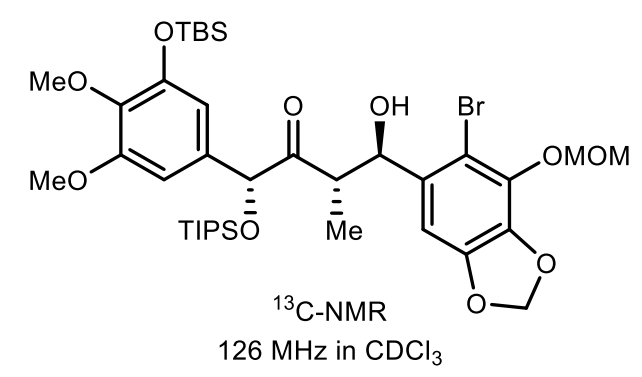

$126 \mathrm{MHz}$ in $\mathrm{CDCl}_{3}$

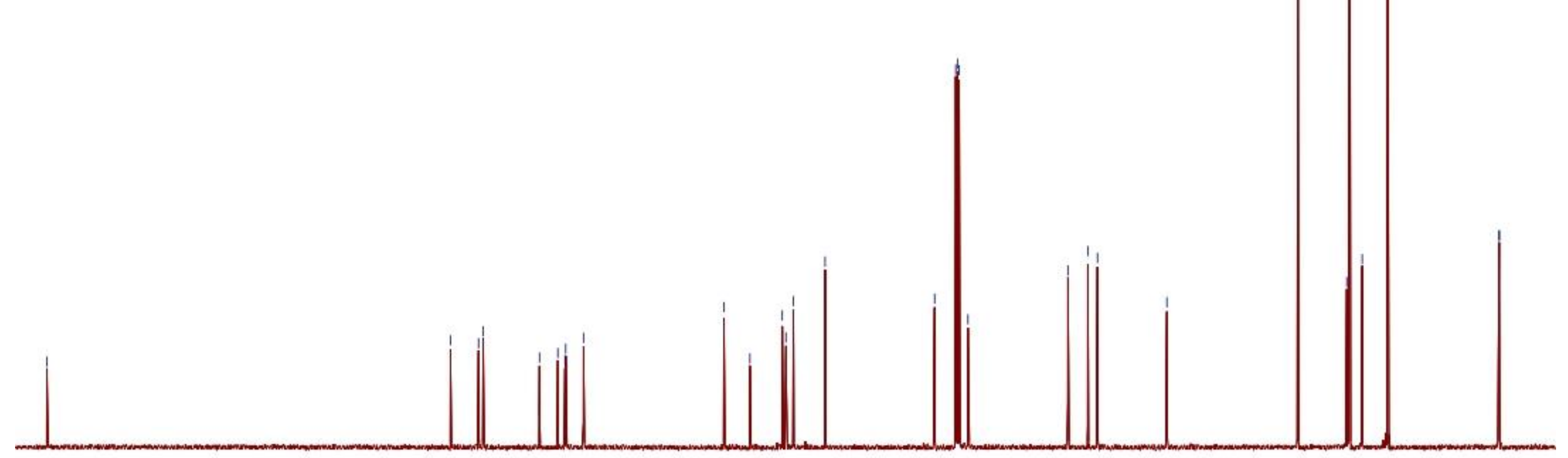




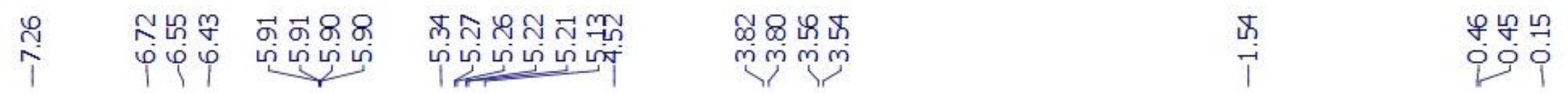

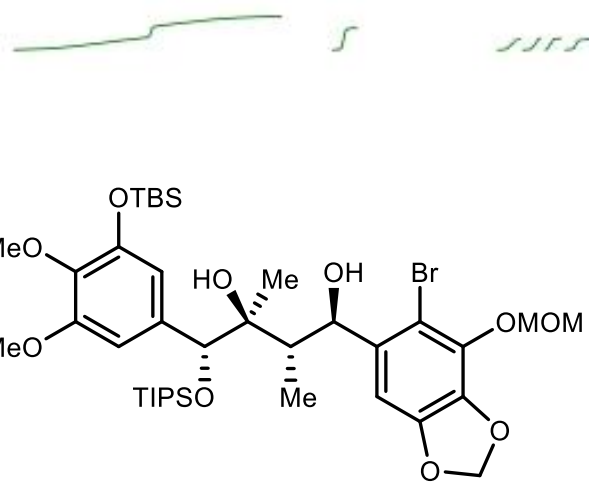

${ }^{1} \mathrm{H}-\mathrm{NMR}$

$500 \mathrm{MHz}$ in $\mathrm{CDCl}_{3}$

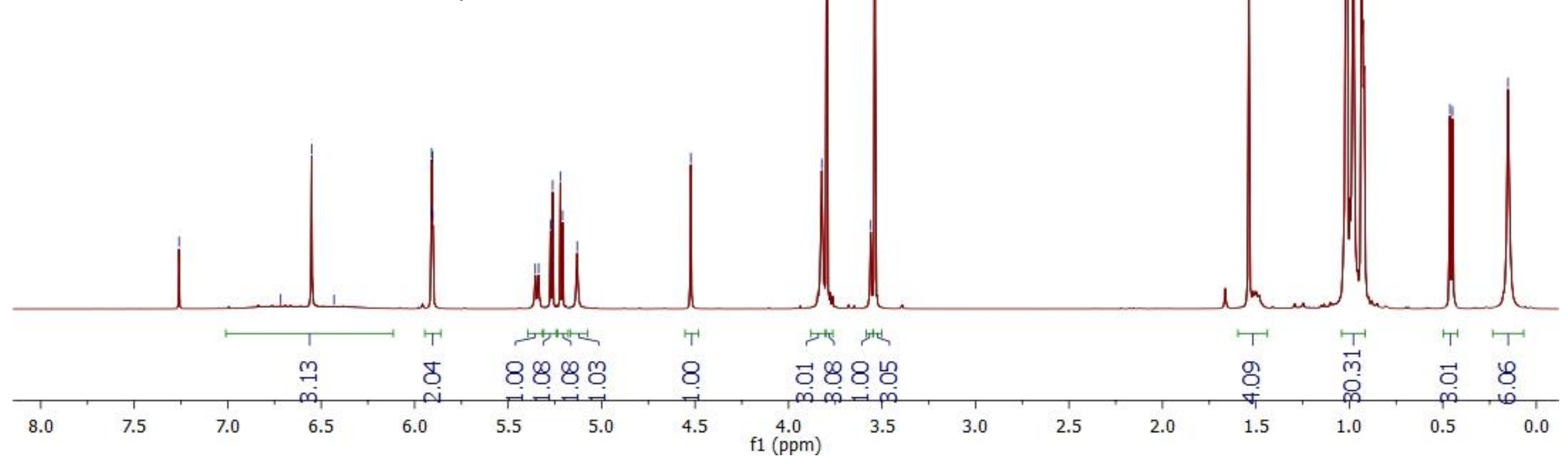


ले 8 퐁ㅇㅇㅛ

贶

\%

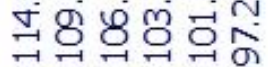

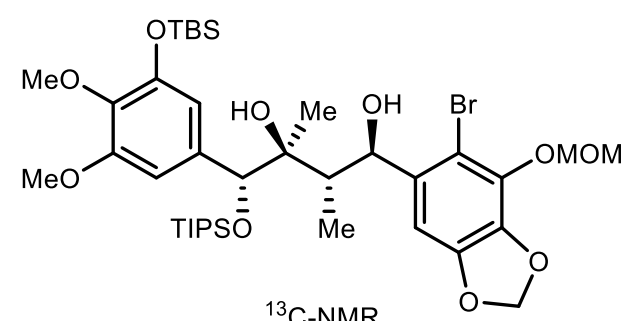

$126 \mathrm{MHz}$ in $\mathrm{CDCl}_{3}$

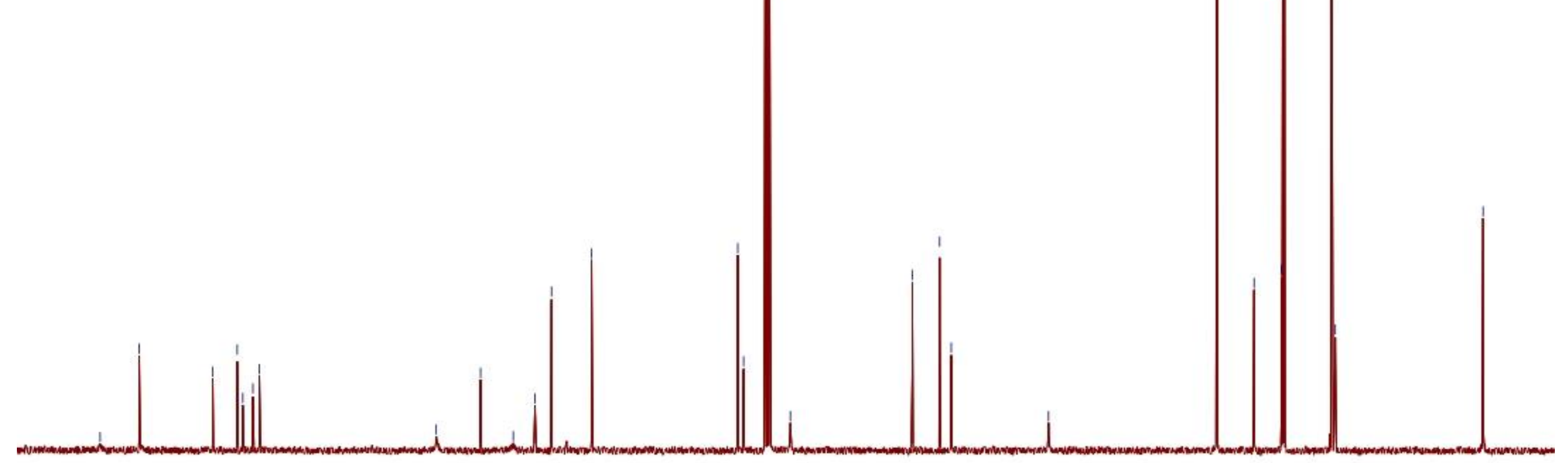


등도용 8 \%

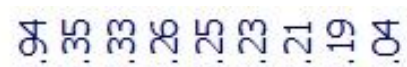

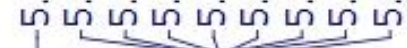

๓ํํํำ

mimm

1) 1 ?

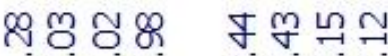

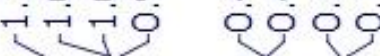

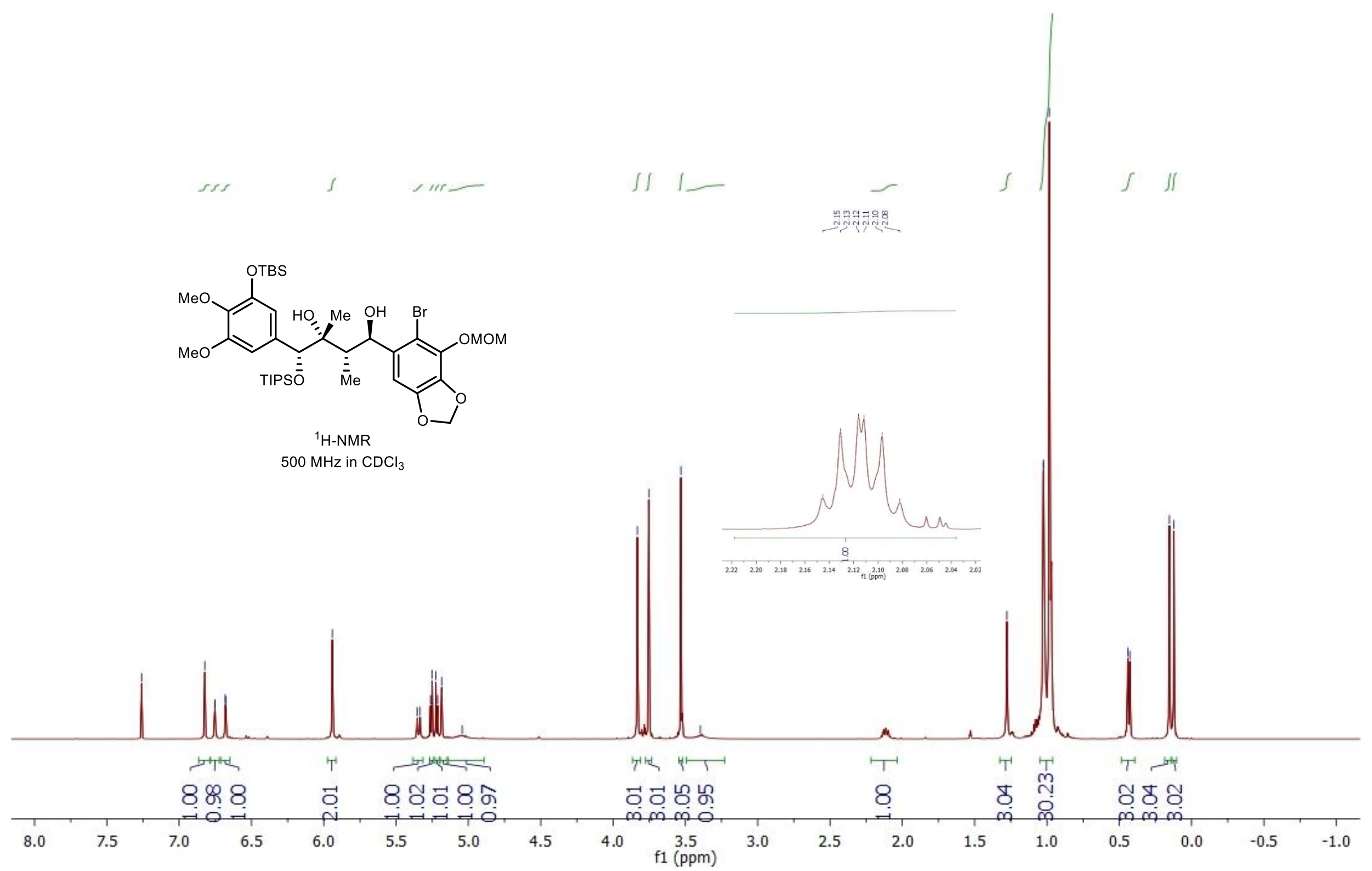


のฺ구요

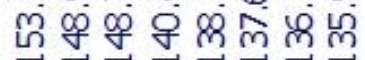
가가각
นกำ

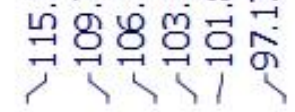

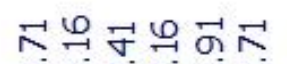

बiRN
กิำำ

员电
쇼ㅇㅛㅛ응요요

นู่สำ

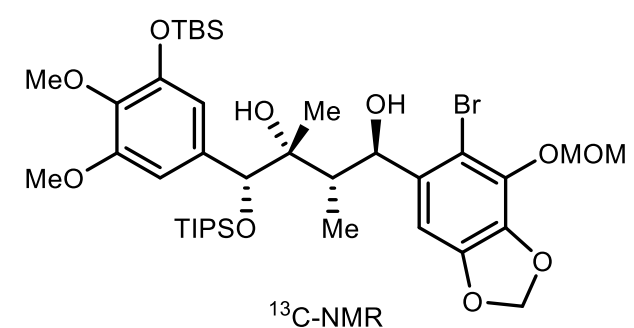

$126 \mathrm{MHz}$ in $\mathrm{CDCl}_{3}$

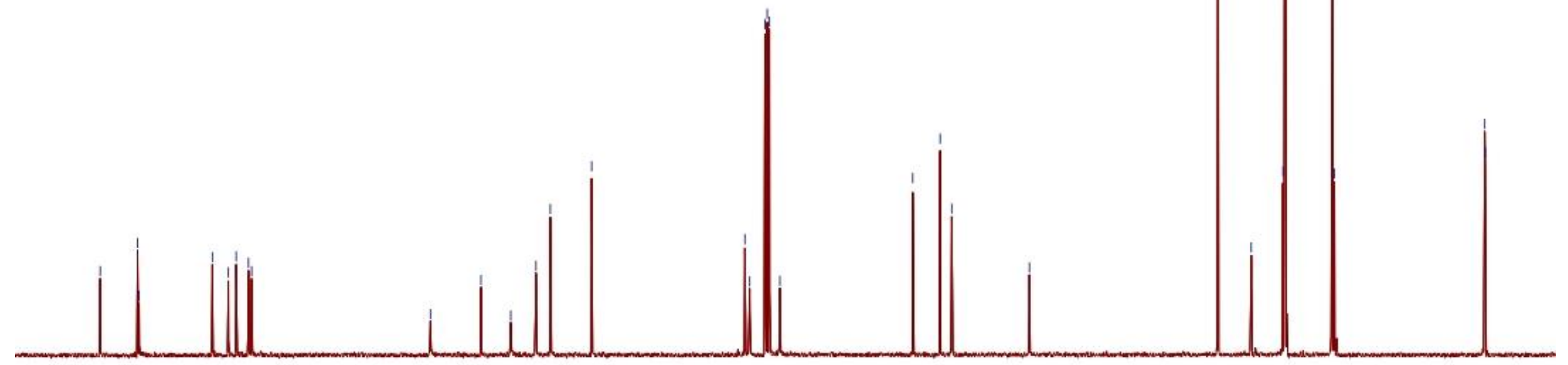




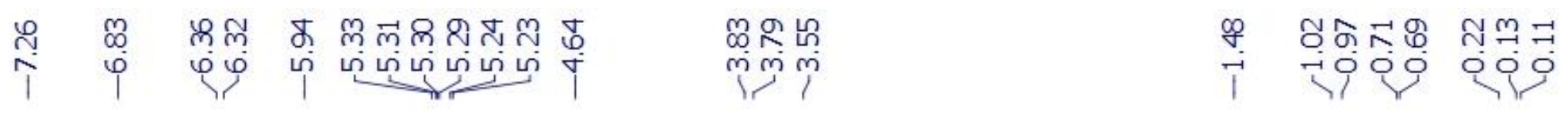

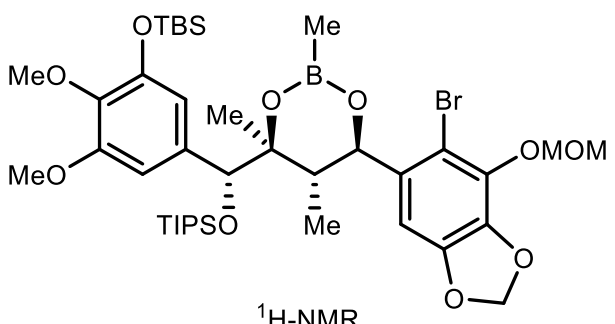

${ }^{1} \mathrm{H}-\mathrm{NMR}$

$500 \mathrm{MHz}$ in $\mathrm{CDCl}_{3}$

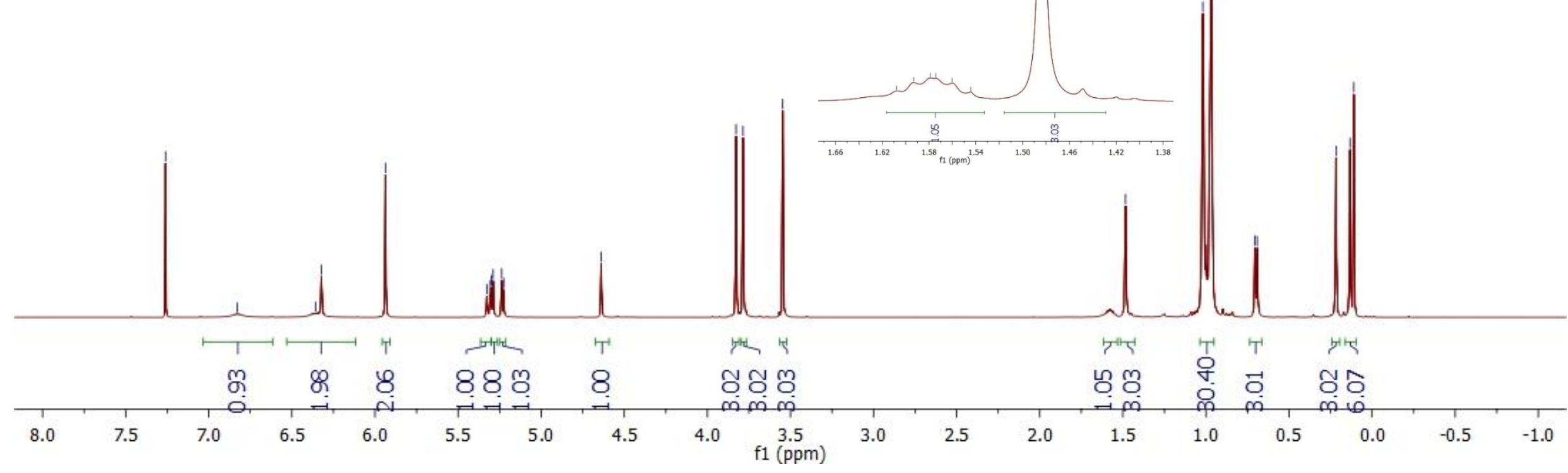


능의

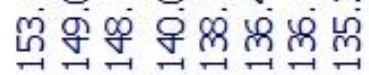

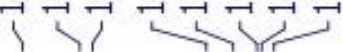

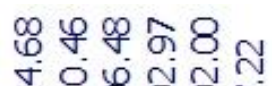

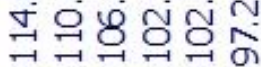

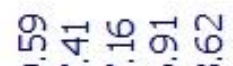
䠌员
ํํํ내요

ชิธ่ำ i

ก

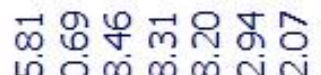

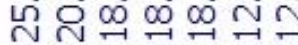

ஜํำ

PT

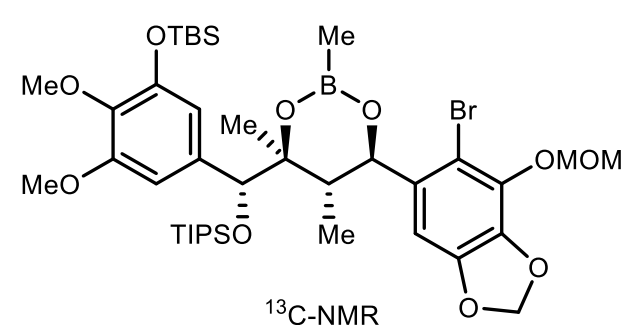

$126 \mathrm{MHz}$ in $\mathrm{CDCl}_{3}$
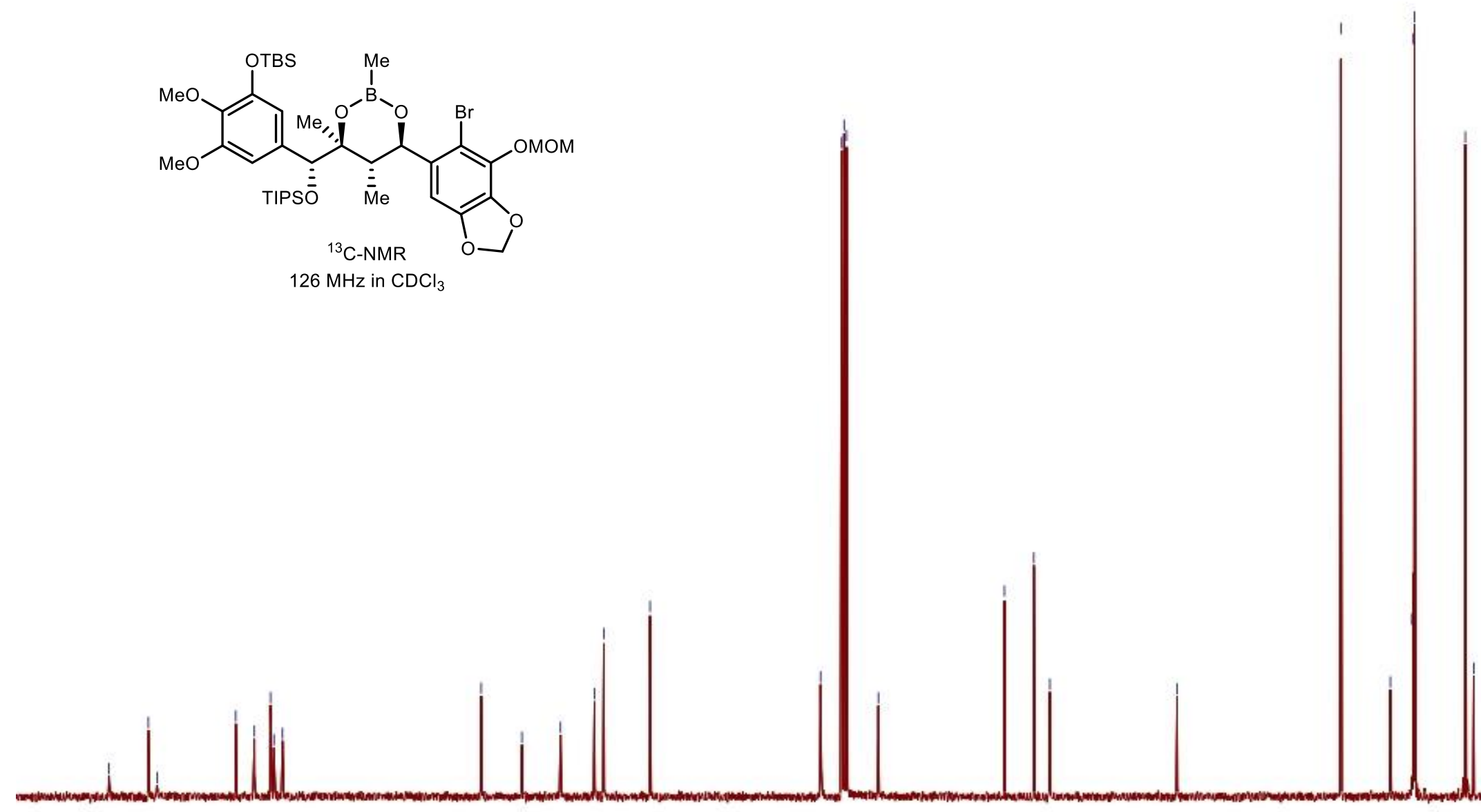


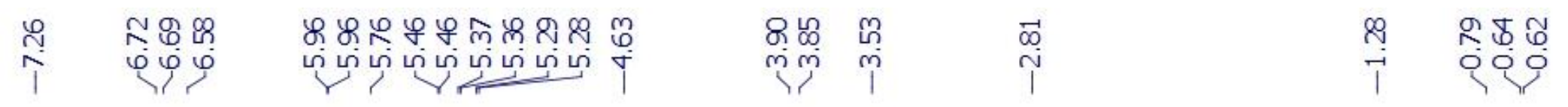

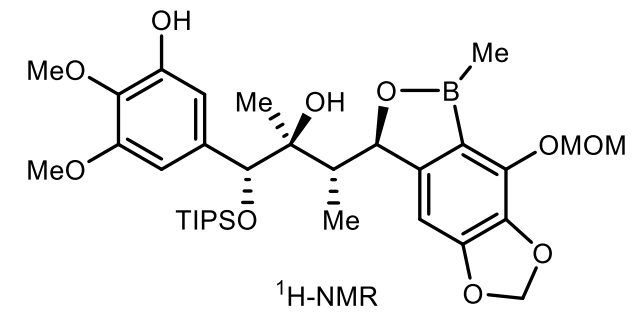

$500 \mathrm{MHz}$ in $\mathrm{CDCl}_{3}$

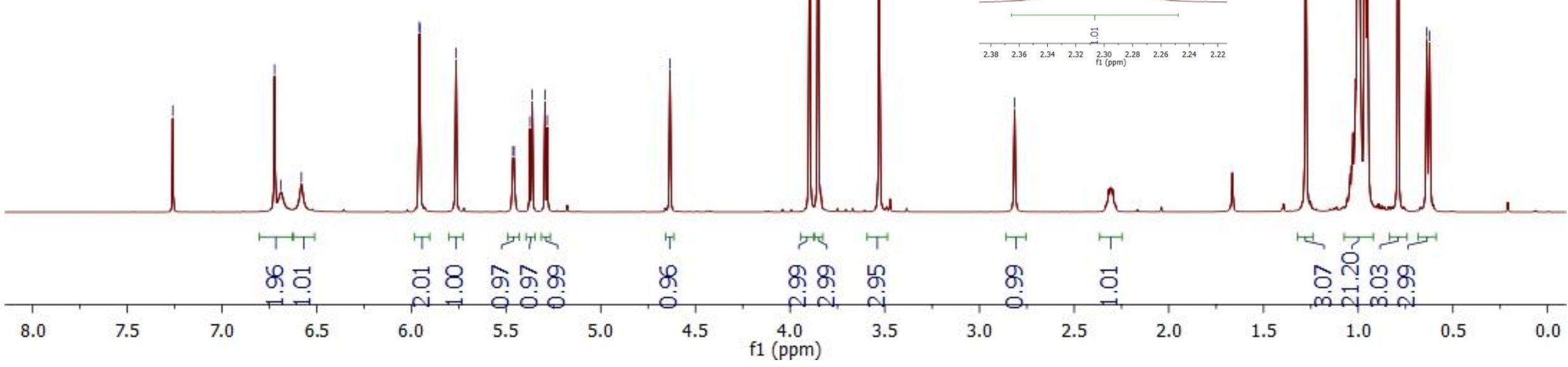



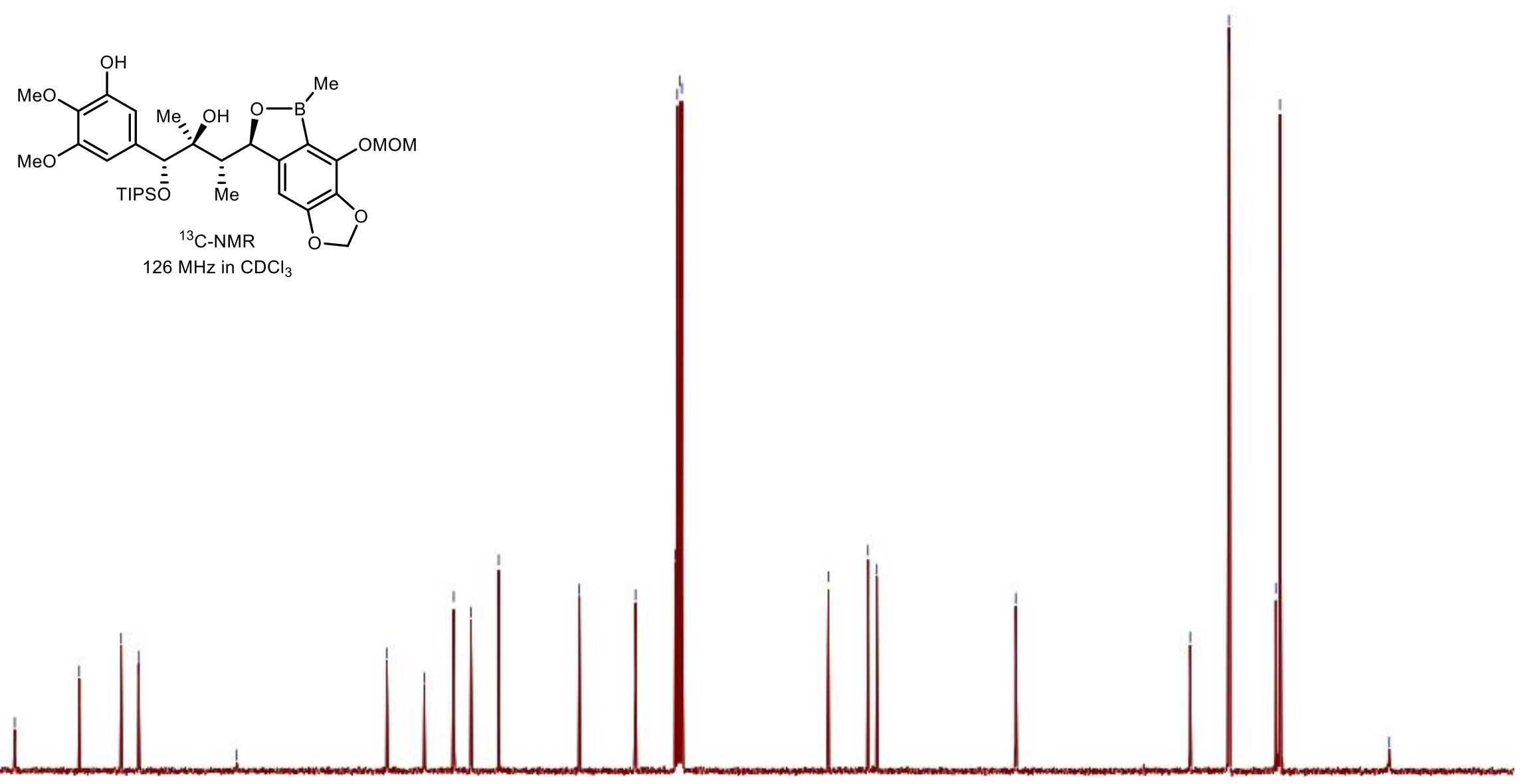


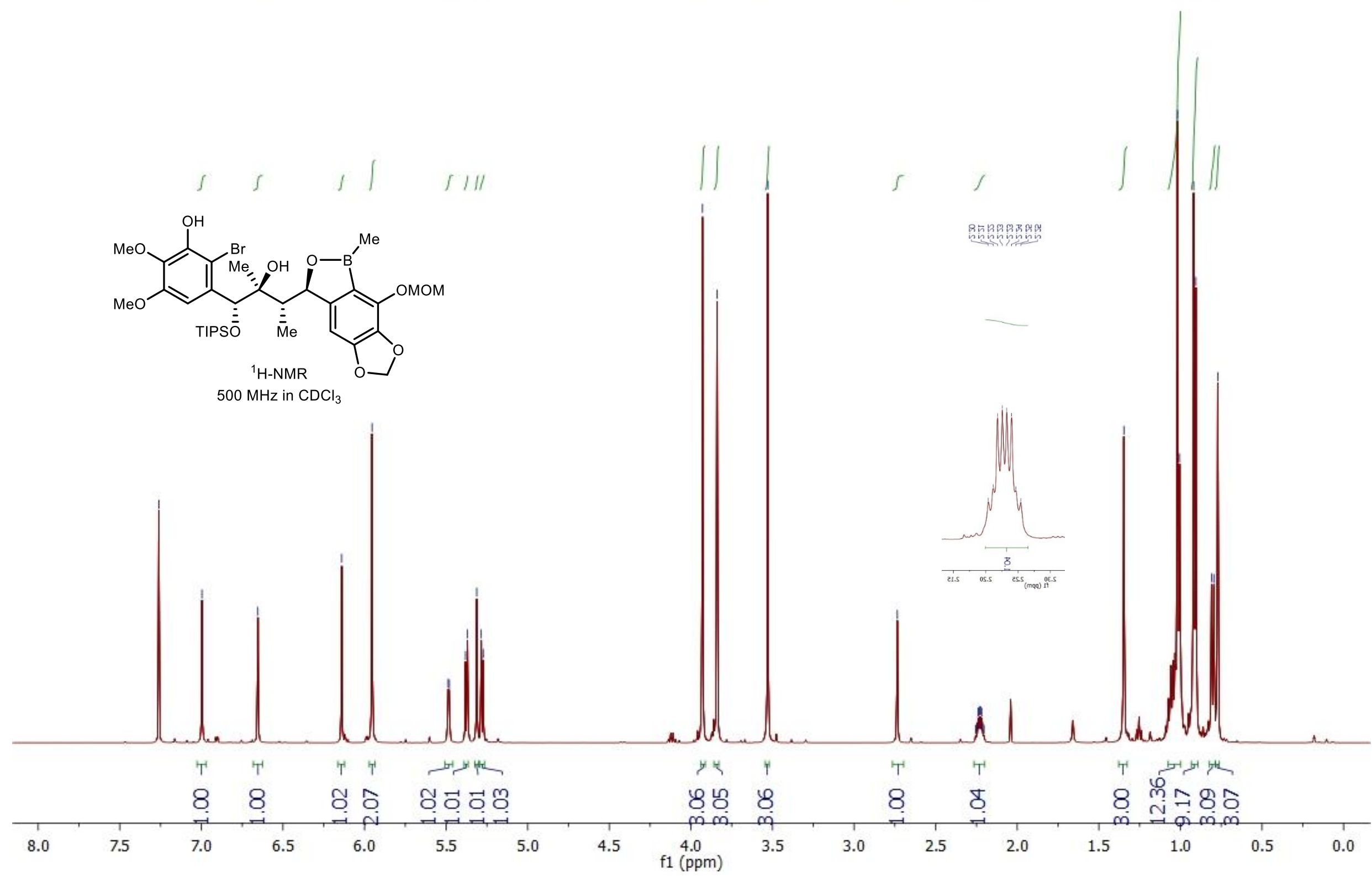


g욕

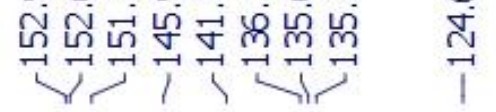

ชூ 8 ํㅓㅇㅇㅛ m ने 8

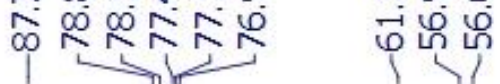
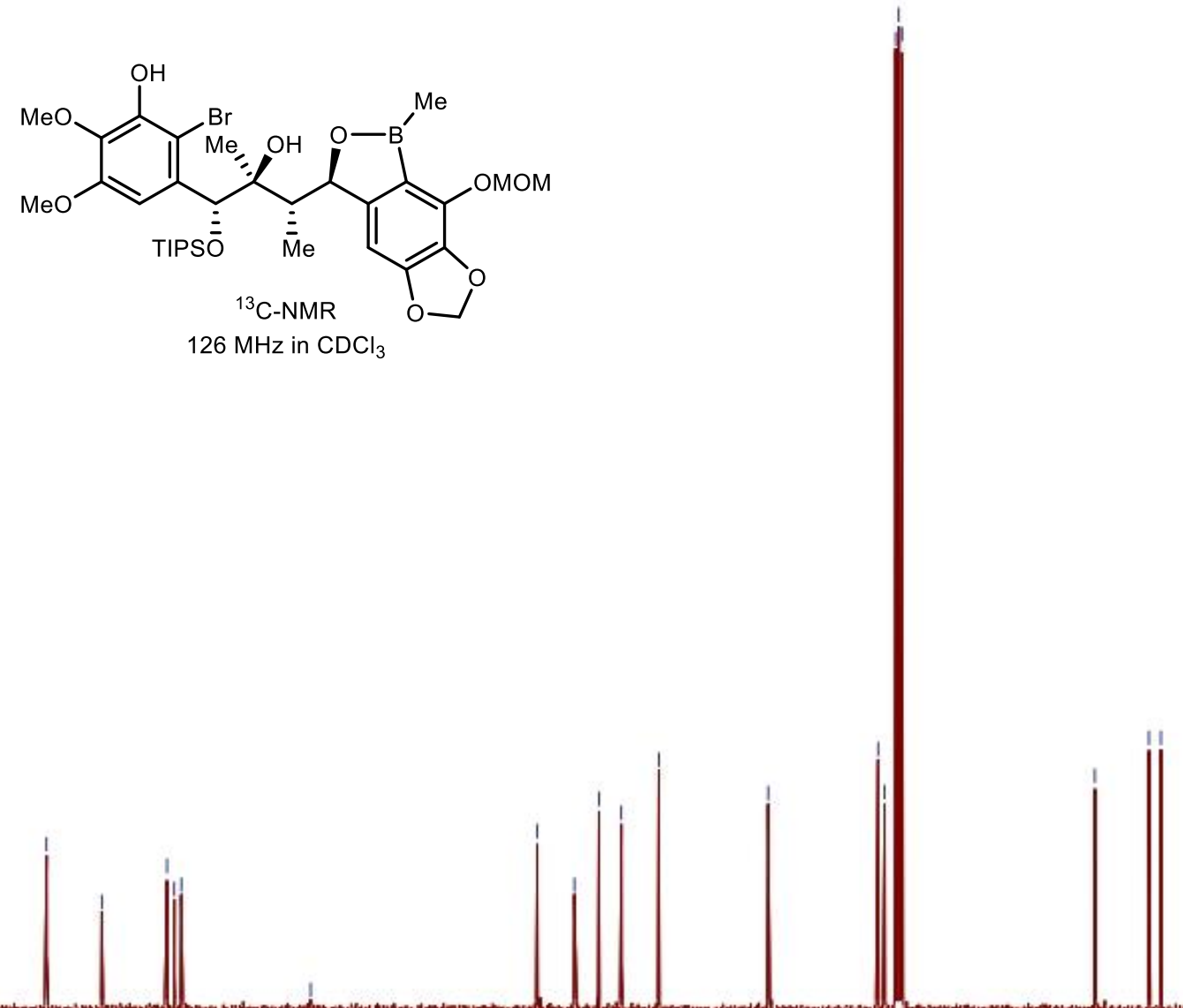


\section{${ }^{1} \mathrm{H}-\mathrm{NMR}$ of compound $\mathbf{3 0}$}

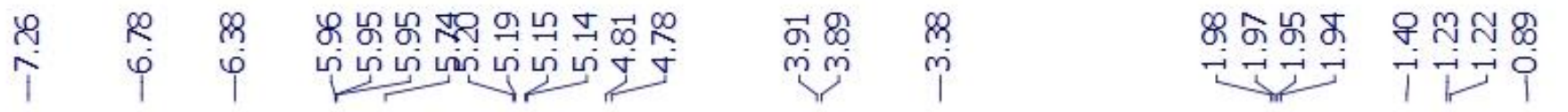

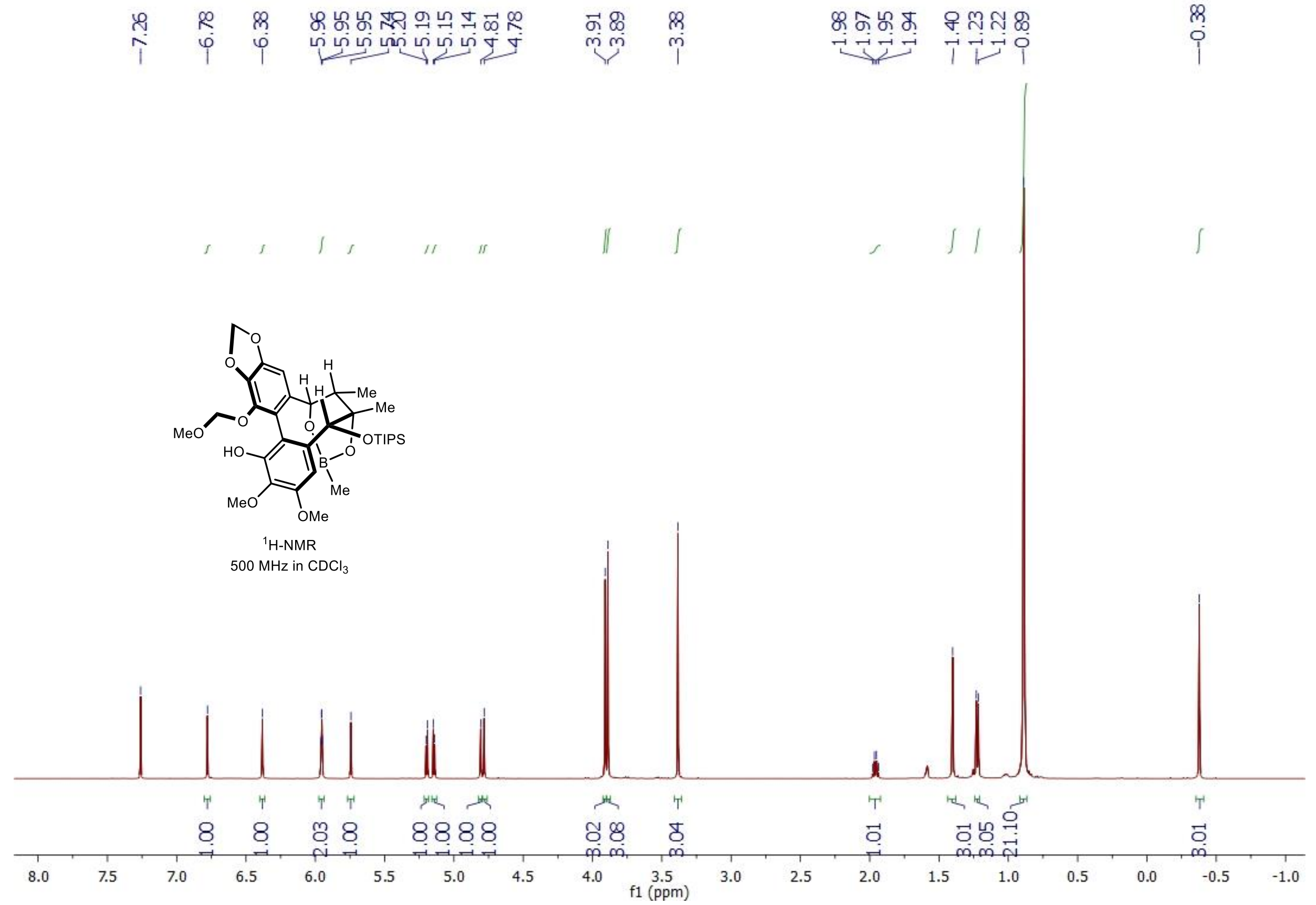


ニ 8 \& 8 g

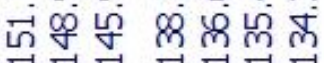
ज杪

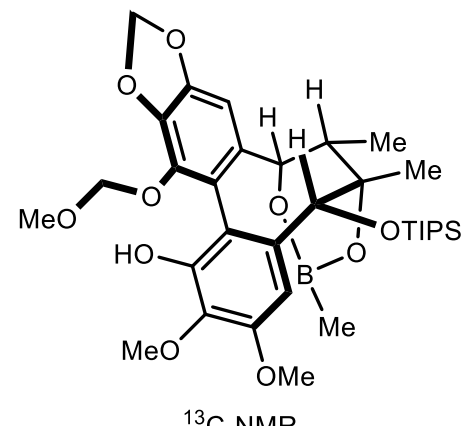

$126 \mathrm{MHz}$ in $\mathrm{CDCl}_{3}$

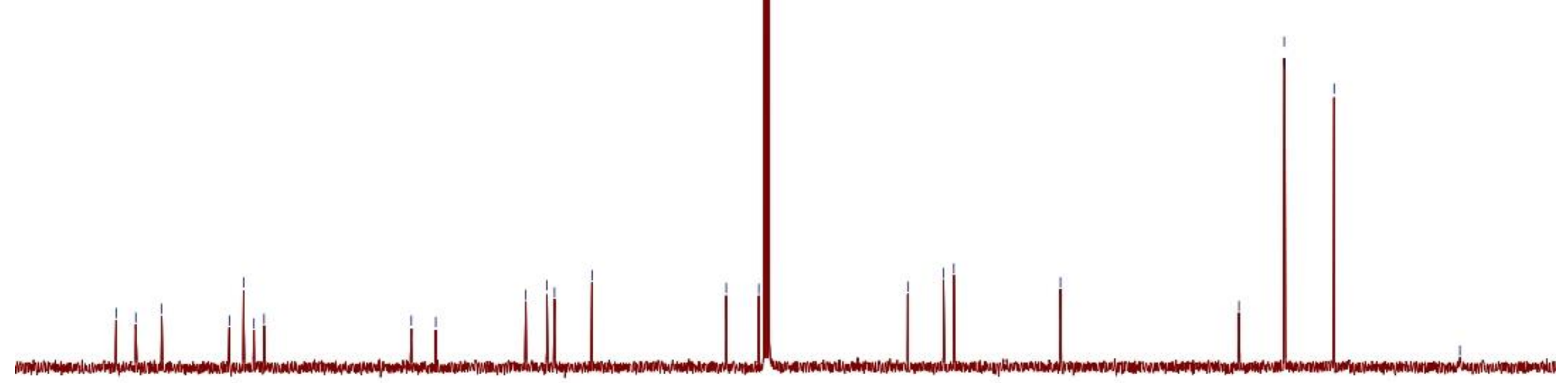




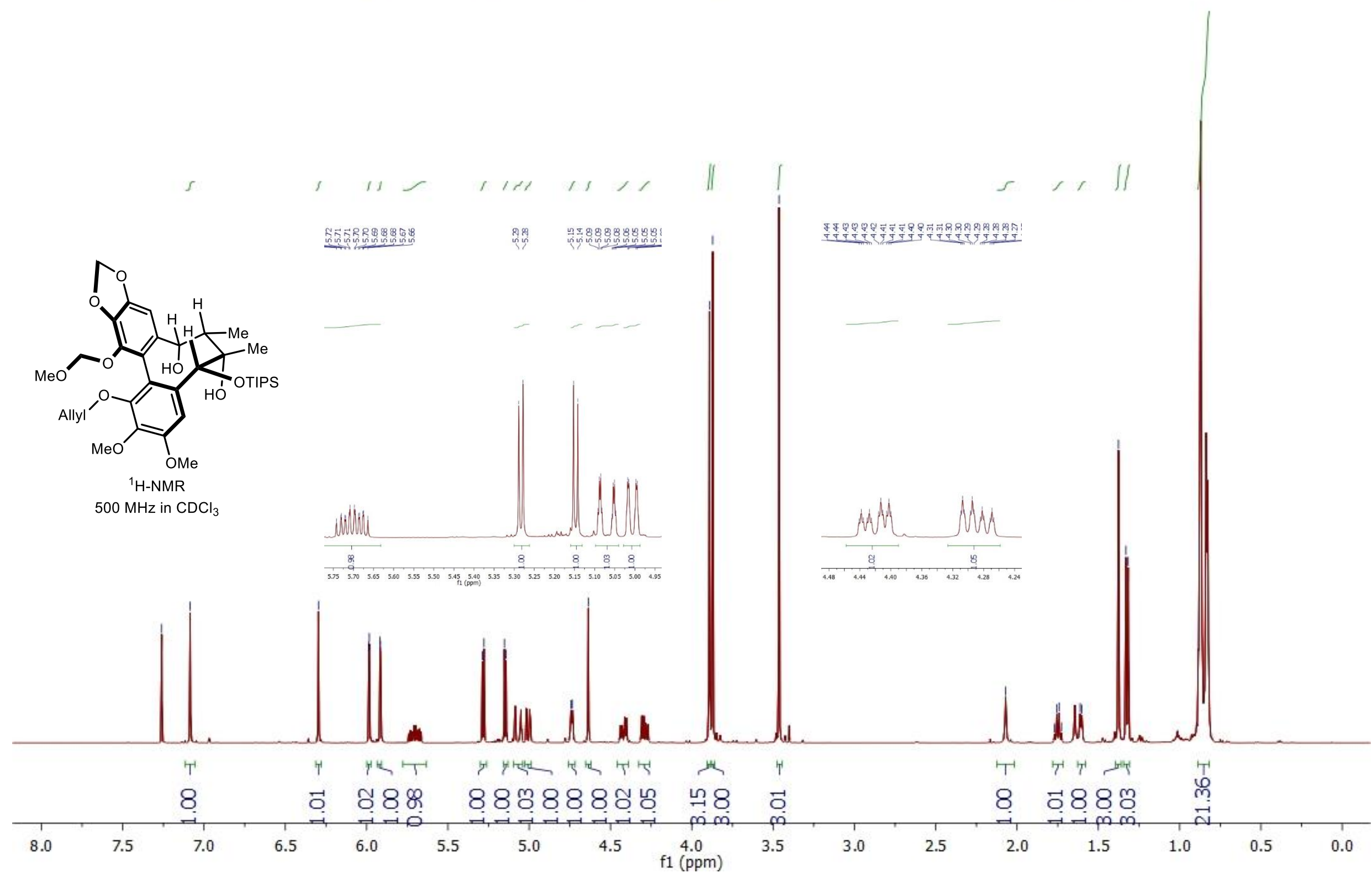




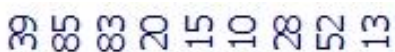
กิ่ कo

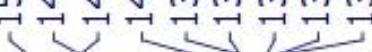

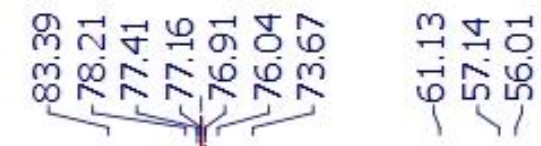

市

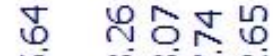

峲

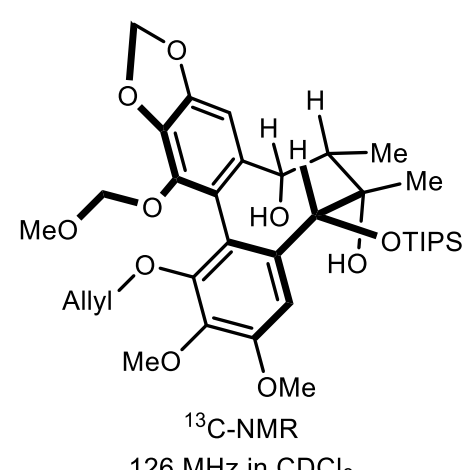

$126 \mathrm{MHz}$ in $\mathrm{CDCl}_{3}$

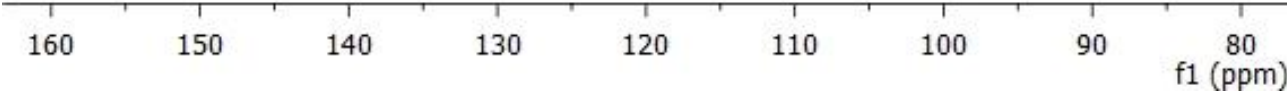

$51 / 77$ 


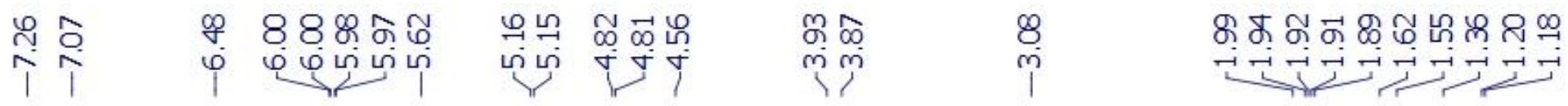

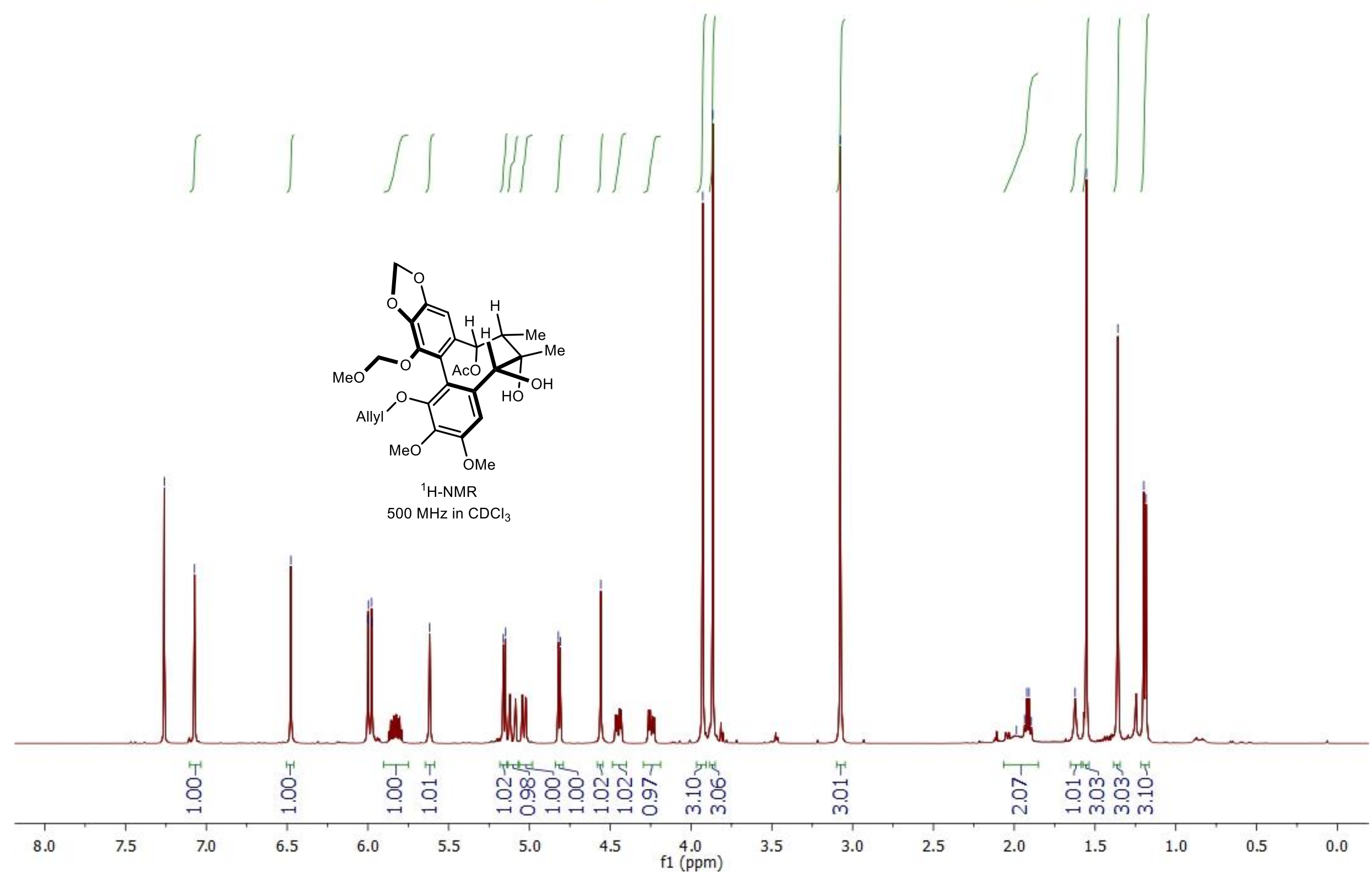




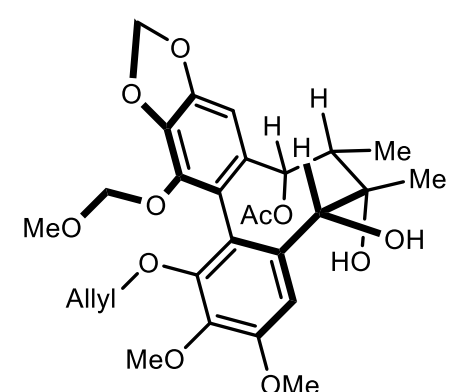

${ }^{13} \mathrm{C}-\mathrm{NMR}$

$126 \mathrm{MHz}$ in $\mathrm{CDCl}_{3}$ 
${ }^{1} \mathrm{H}-\mathrm{NMR}$ of compound 31

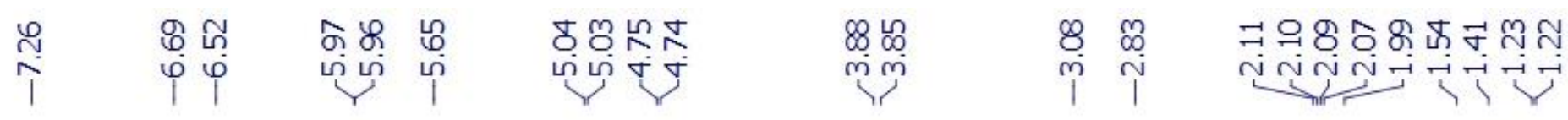

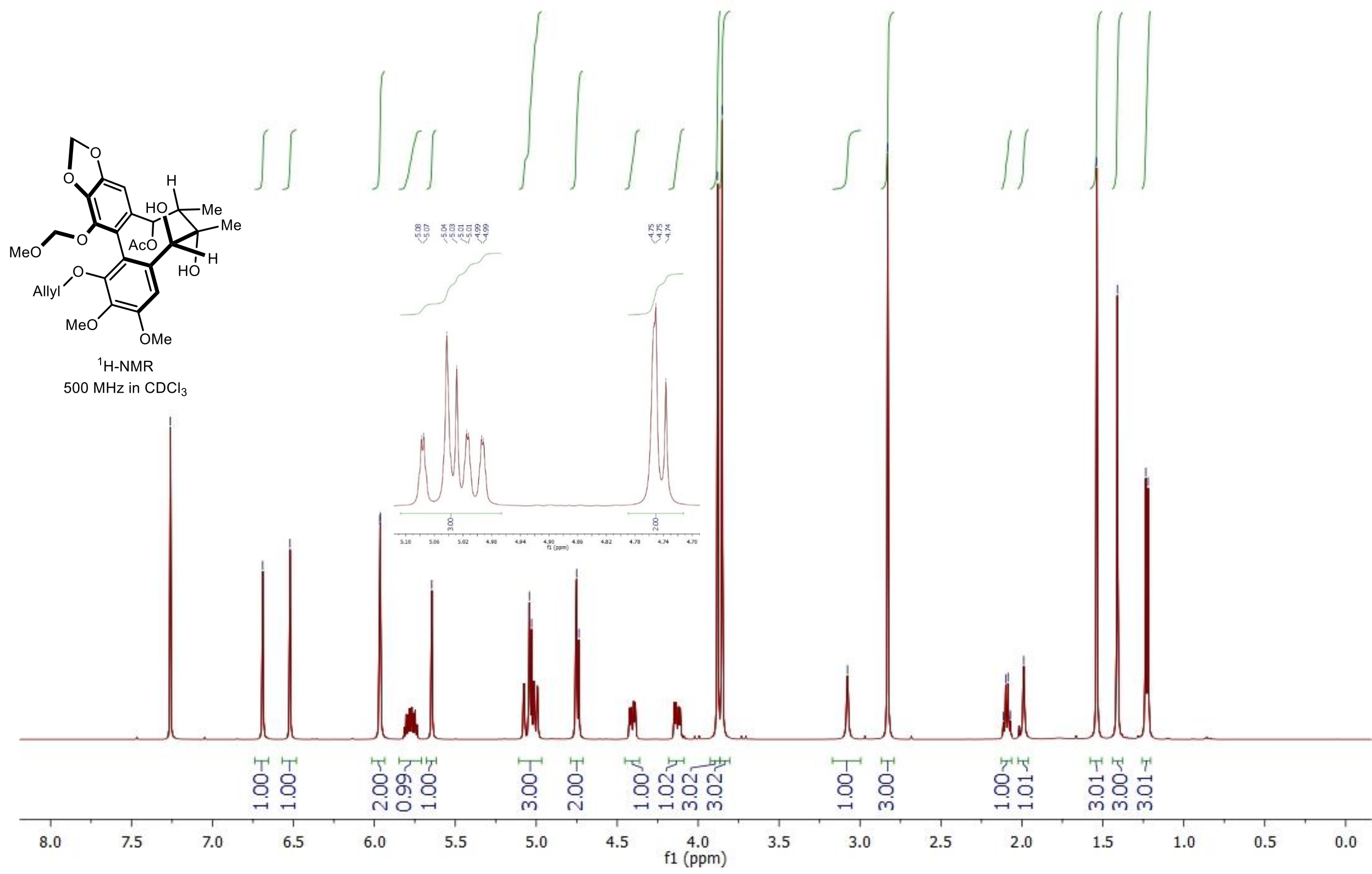

54 / 77 


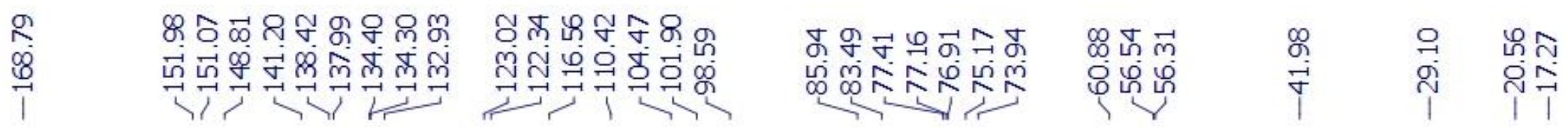

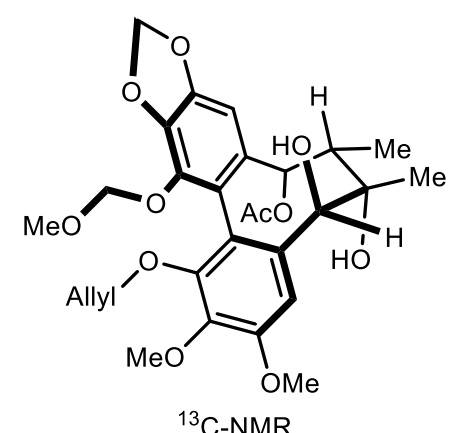

${ }^{13} \mathrm{C}-\mathrm{NMR}$

$126 \mathrm{MHz}$ in $\mathrm{CDCl}_{3}$
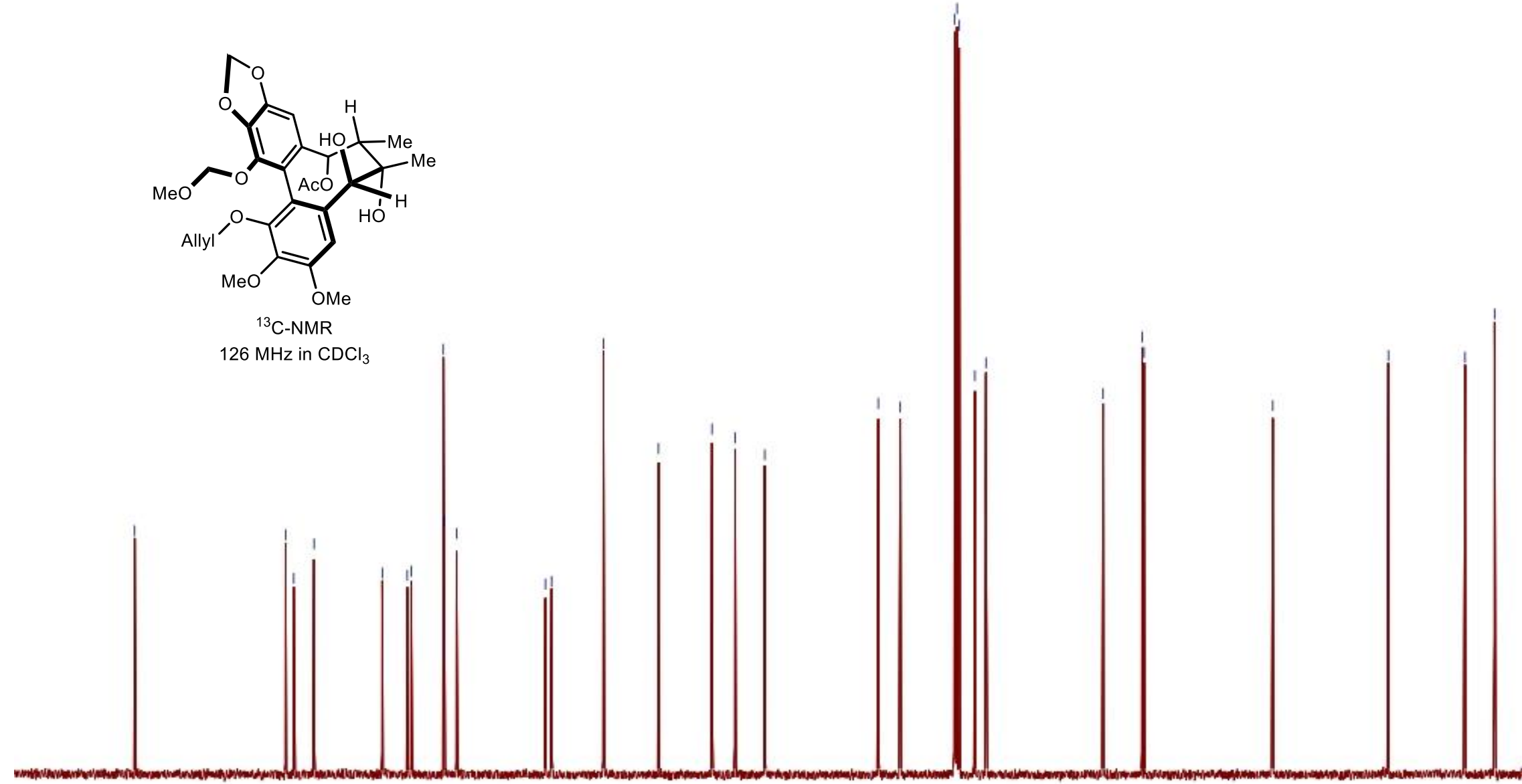


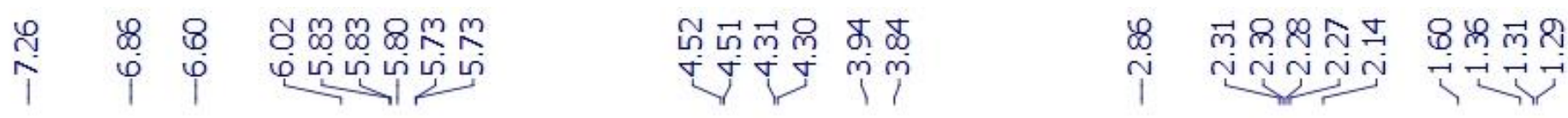

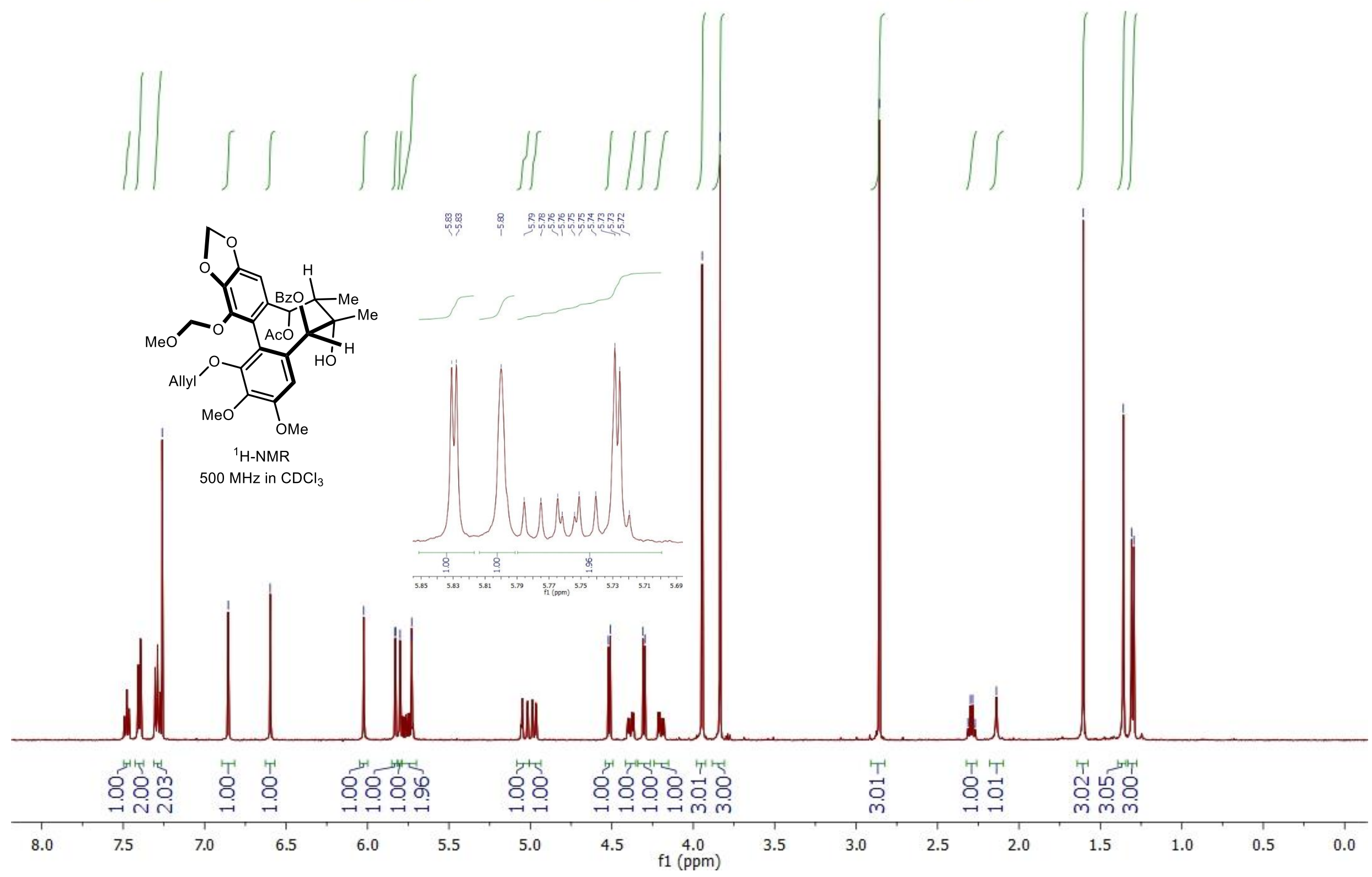

$56 / 77$ 


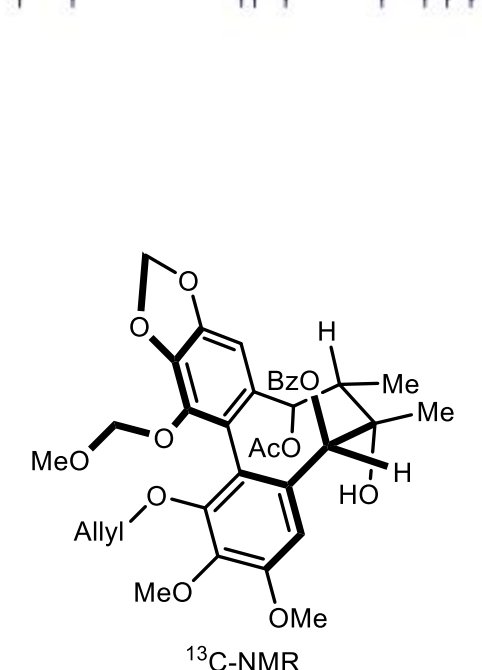

${ }^{13} \mathrm{C}-\mathrm{NMR}$

$126 \mathrm{MHz}$ in $\mathrm{CDCl}_{3}$

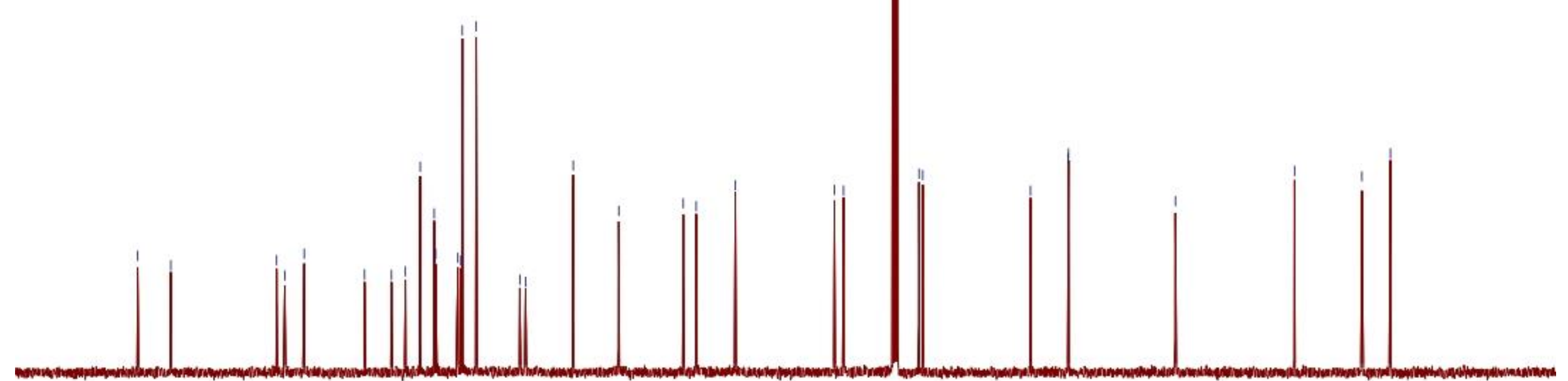




\section{${ }^{1} \mathrm{H}-\mathrm{NMR}$ of compound 32}

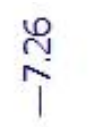

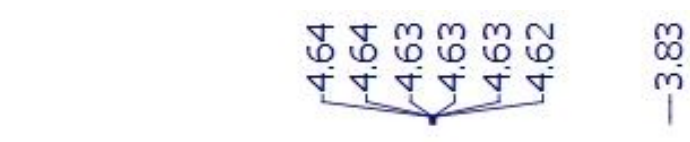

$\prod_{O}^{\mathrm{O}} \overbrace{A l l y l}$

${ }^{1} \mathrm{H}-\mathrm{NMR}$

$500 \mathrm{MHz}$ in $\mathrm{CDCl}_{3}$
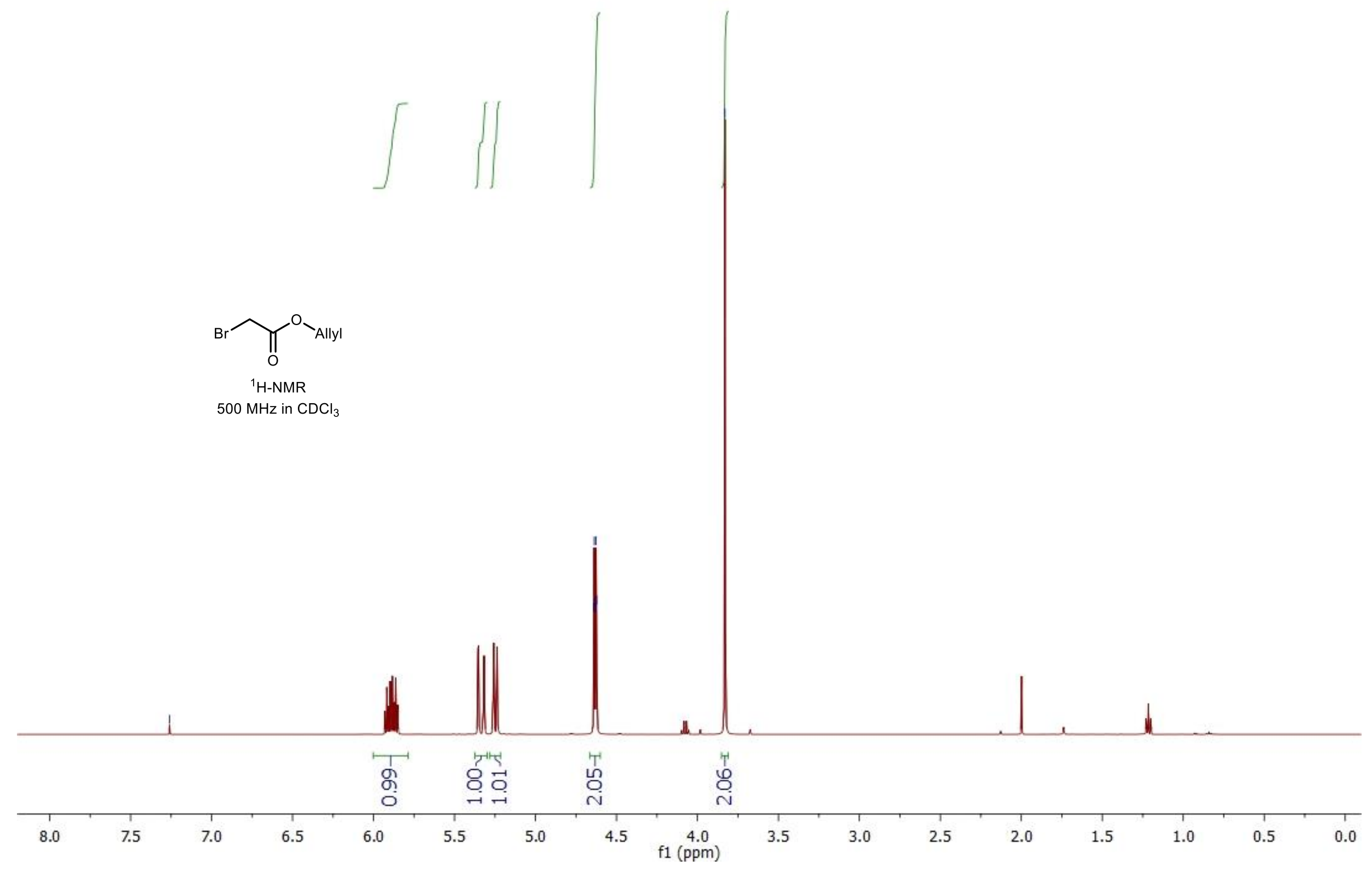

58 / 77 


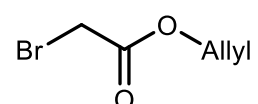

${ }^{13} \mathrm{C}-\mathrm{NMR}$

$126 \mathrm{MHz}$ in $\mathrm{CDCl}_{3}$

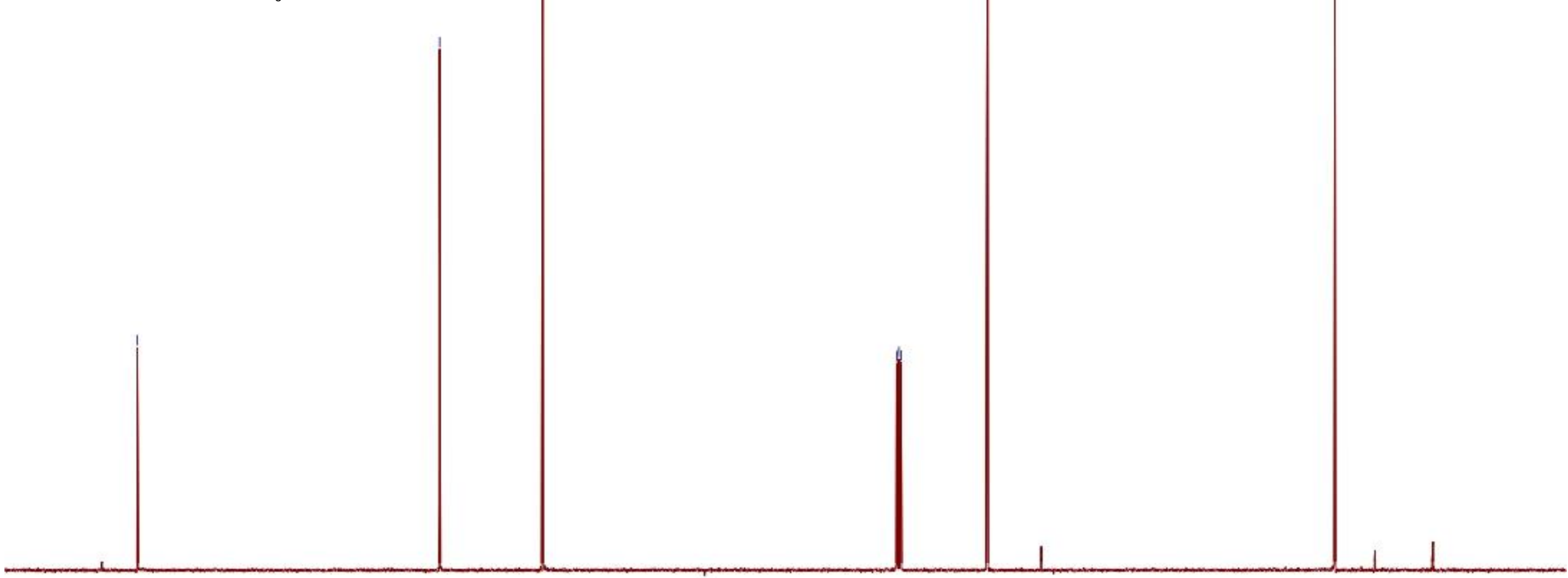




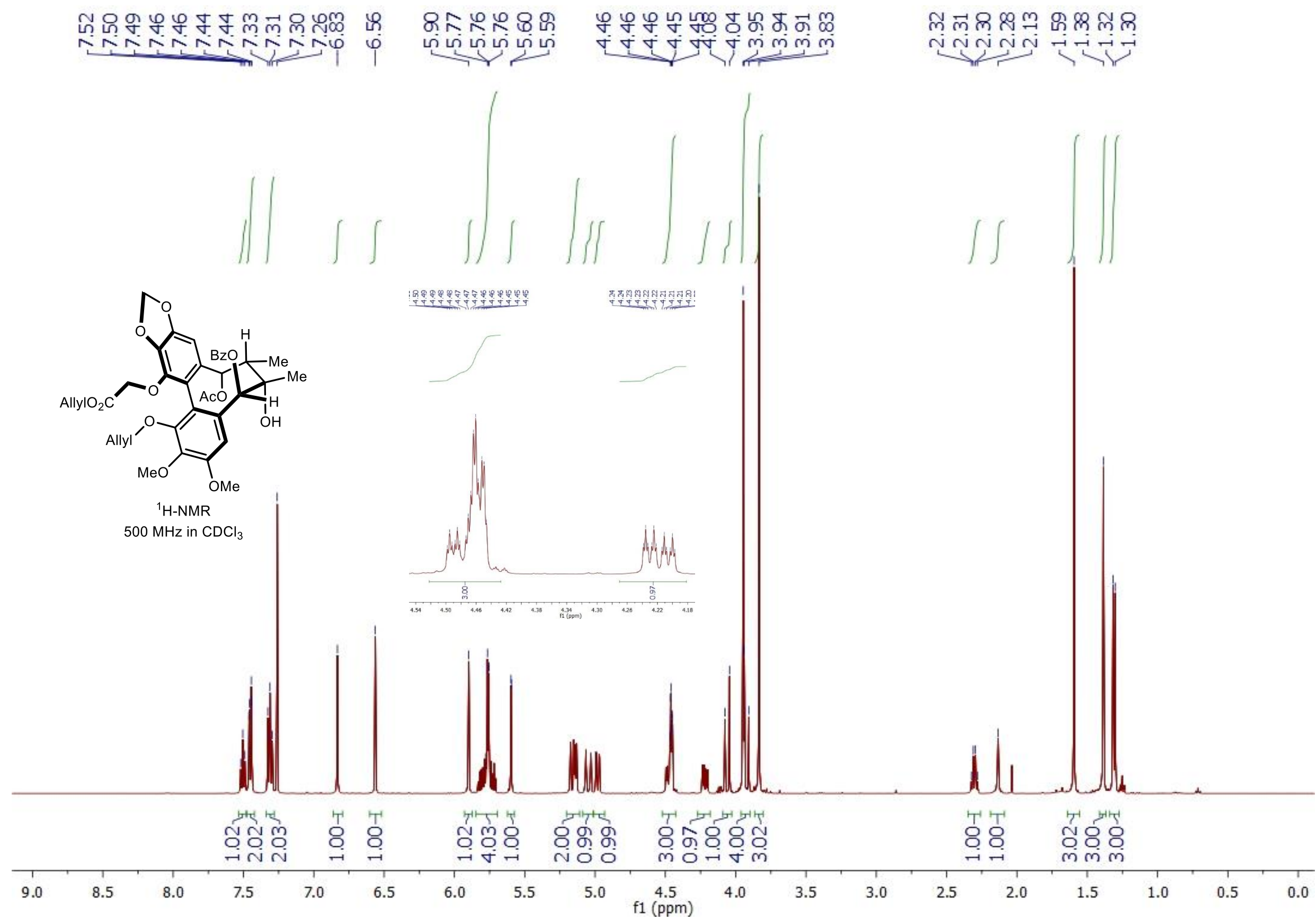

60 / 77 


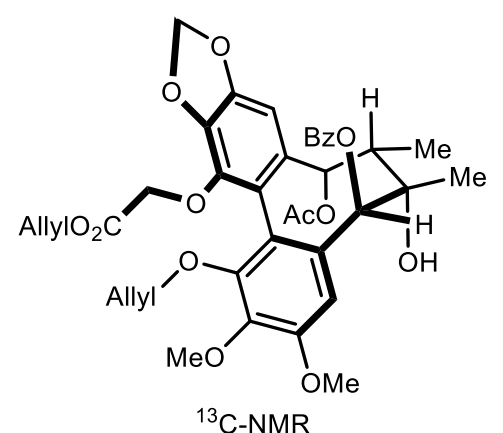

$126 \mathrm{MHz}$ in $\mathrm{CDCl}_{3}$

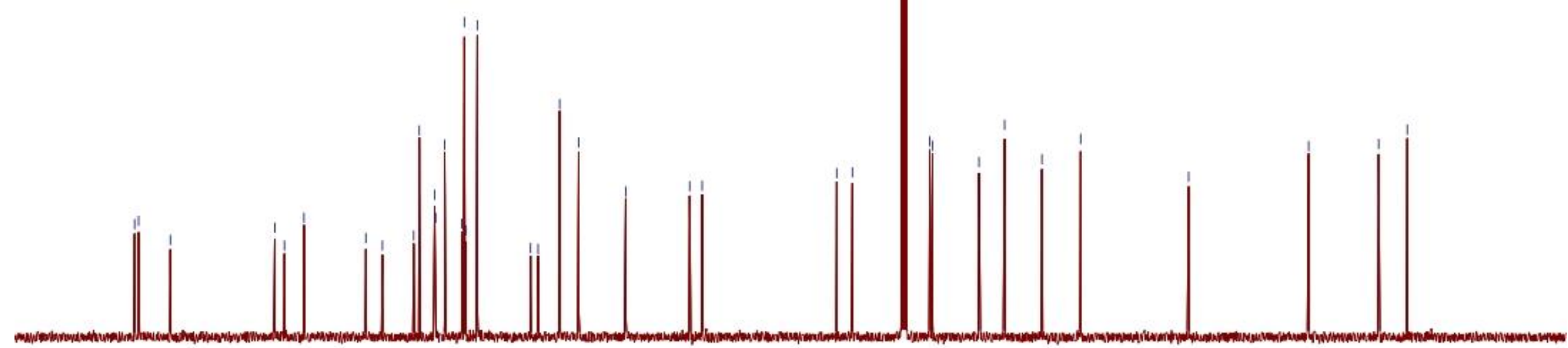




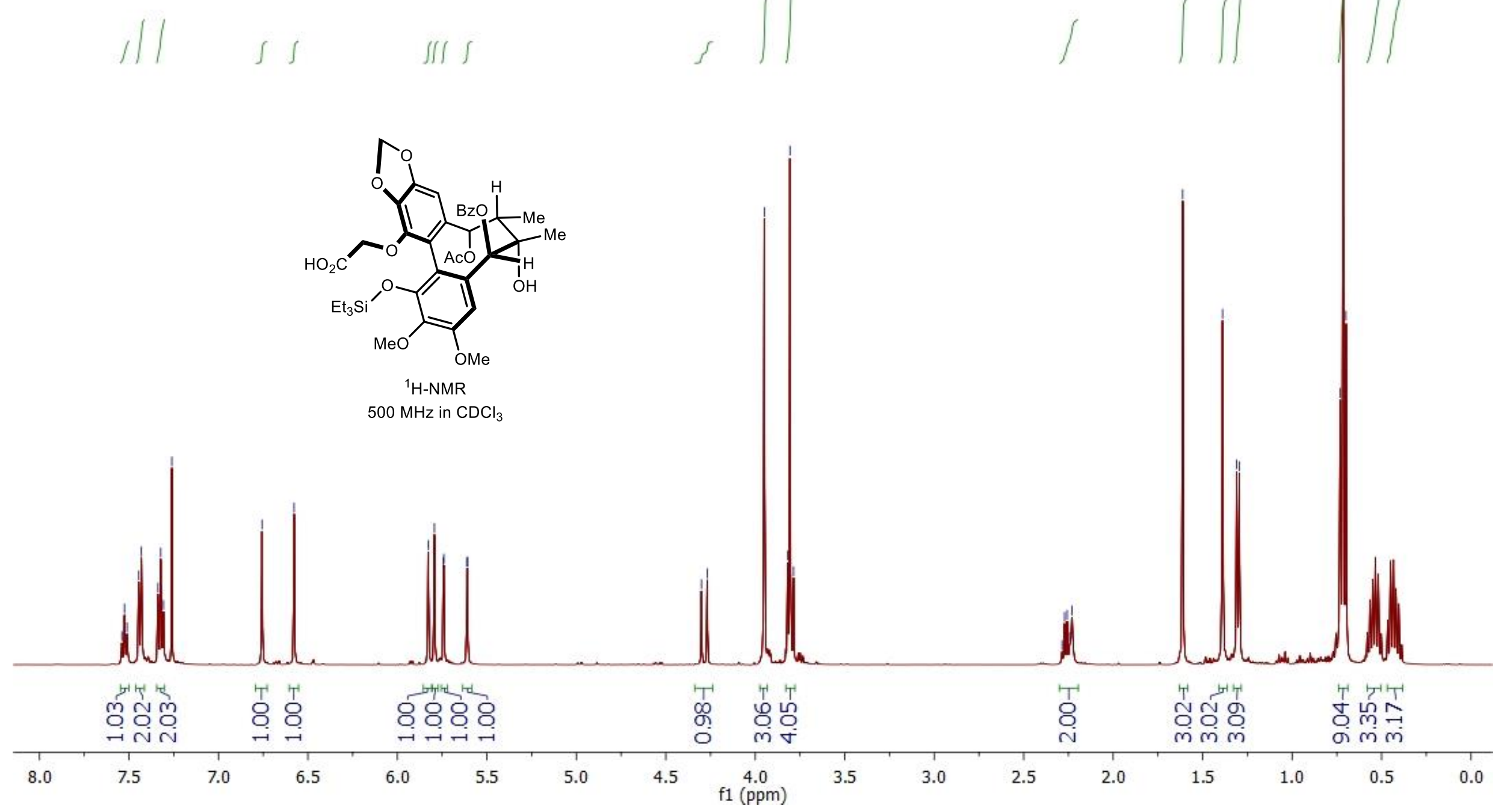


$8 \div 0$ 电要

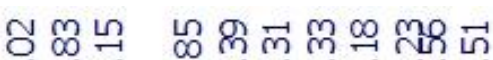

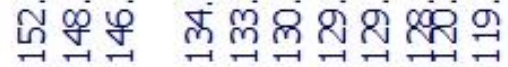
भान

\section{붕ํㅇ 8 엉으}

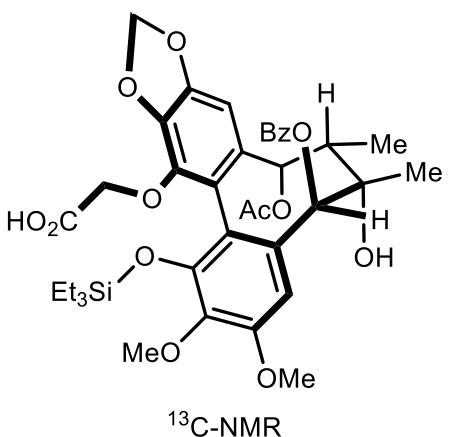

$126 \mathrm{MHz}$ in $\mathrm{CDCl}_{3}$

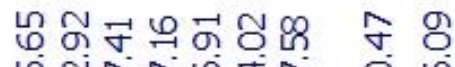

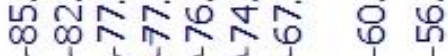

క

$\stackrel{m}{i}$ 


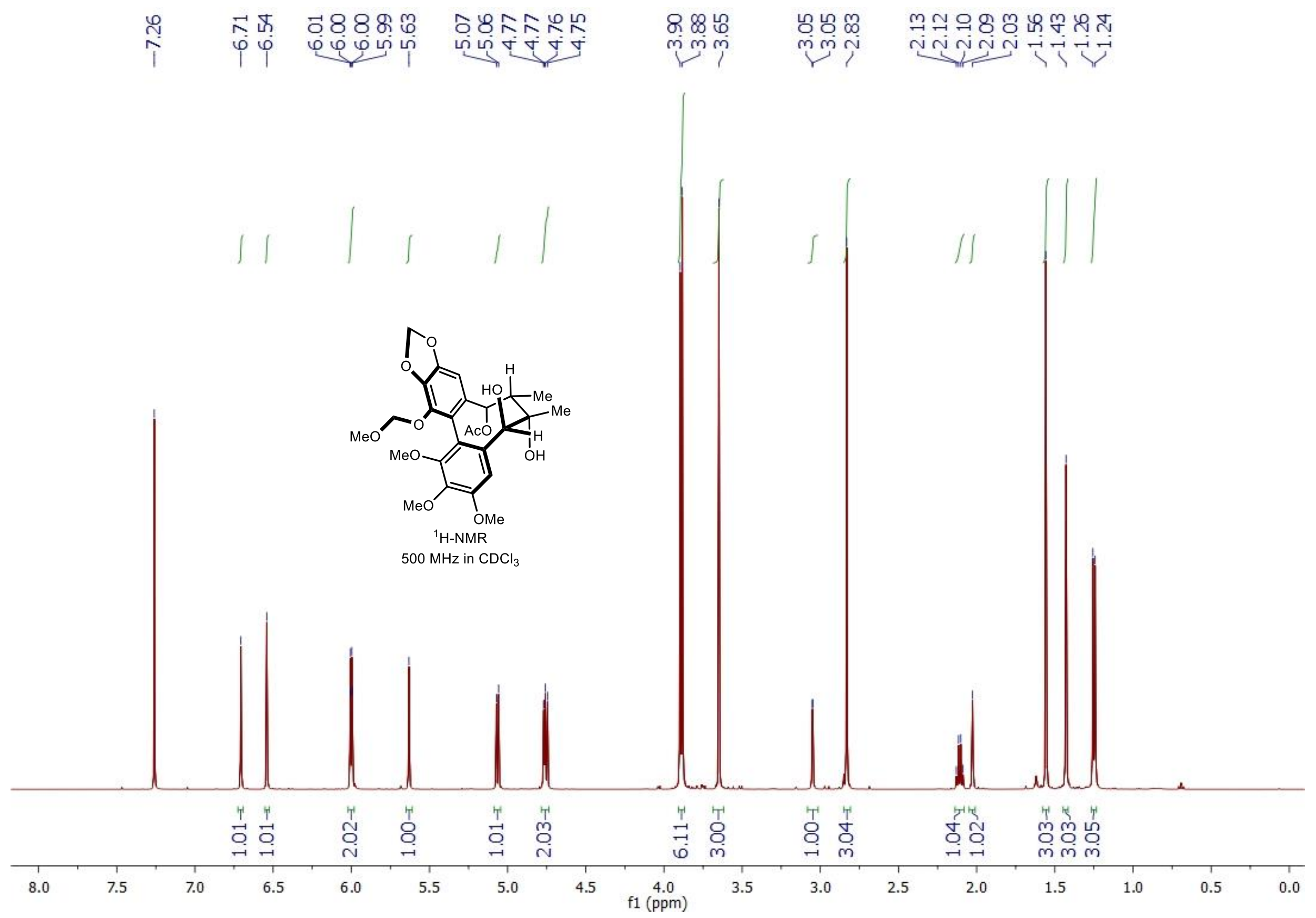

64 / 77 


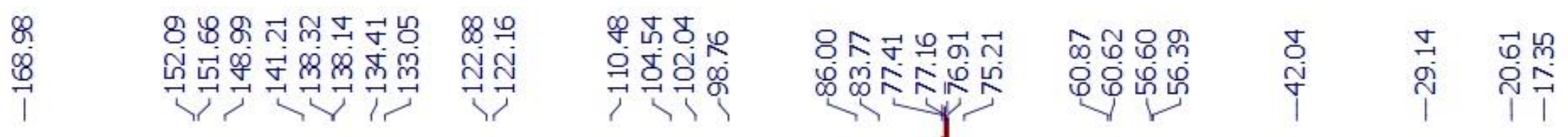

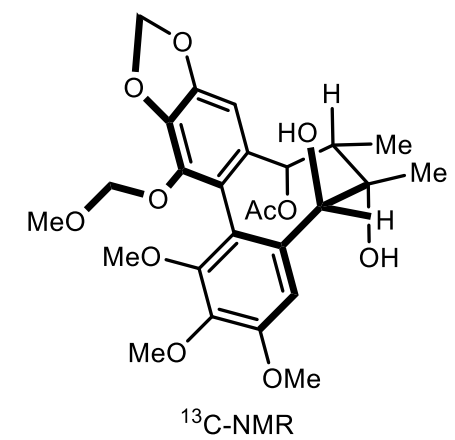

$126 \mathrm{MHz}^{\text {in } \mathrm{CDCl}_{3}}$ 


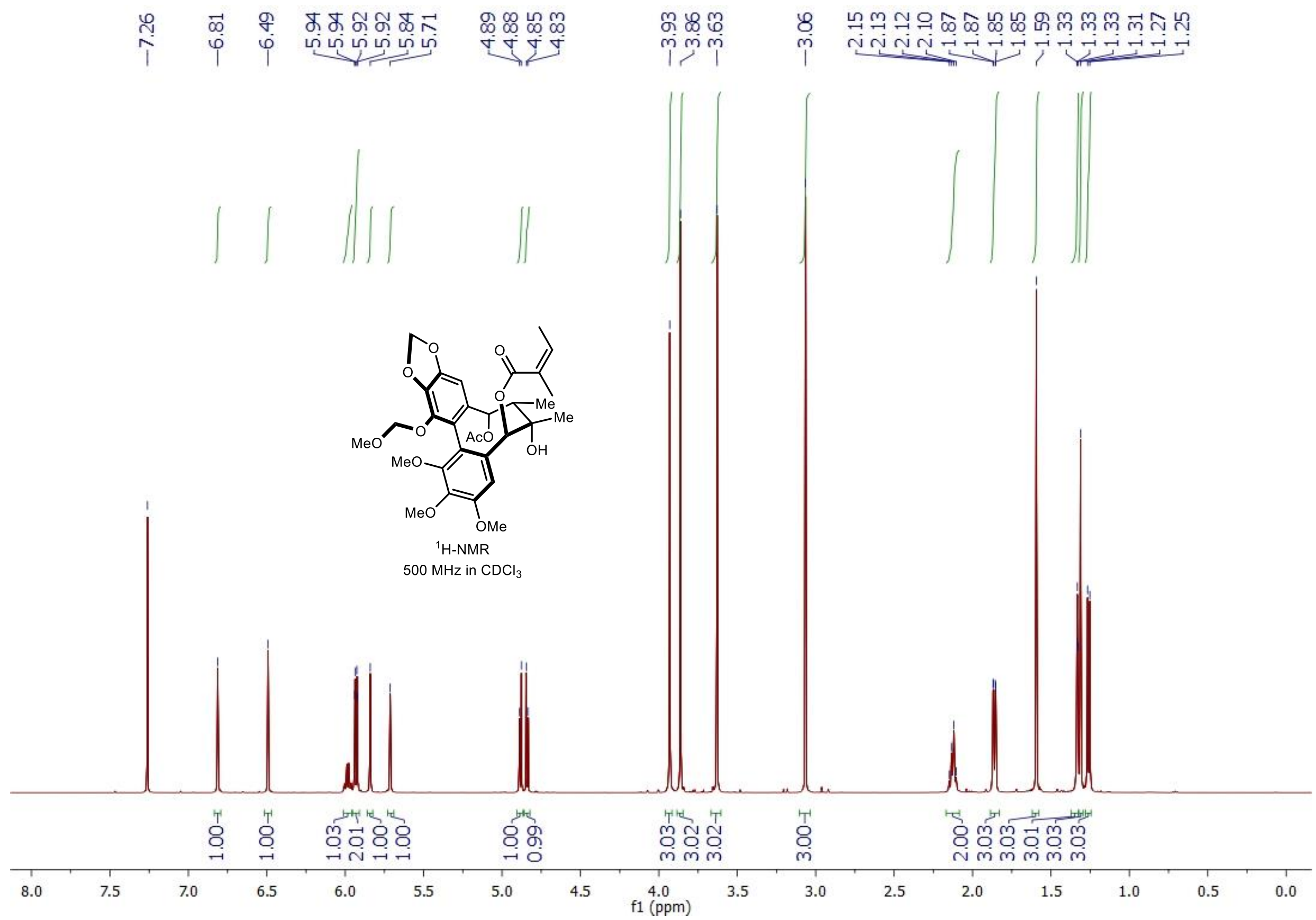

$66 / 77$ 


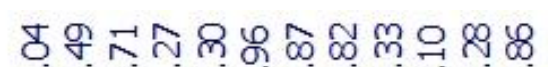

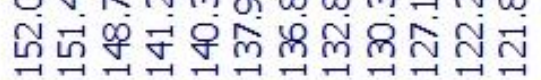

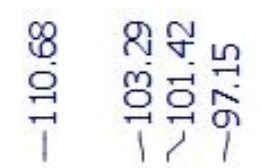

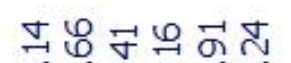

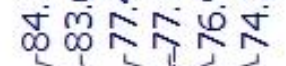

ㄴํำำ

○ำㄴำ

y닌

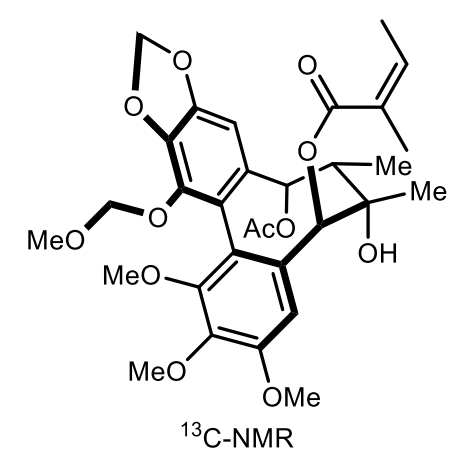

$126 \mathrm{MHz}^{\text {in } \mathrm{CDCl}_{3}}$

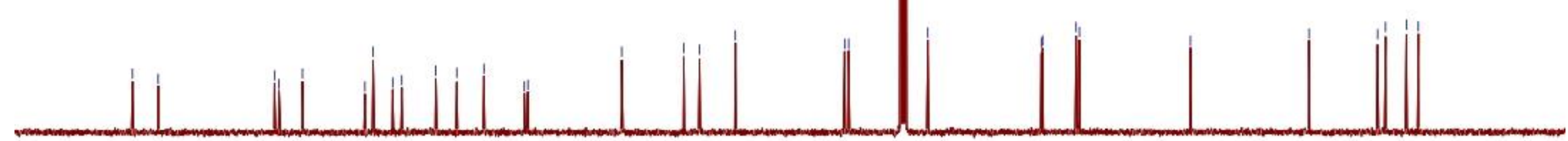




\section{${ }^{1} \mathrm{H}-\mathrm{NMR}$ of compound 35}

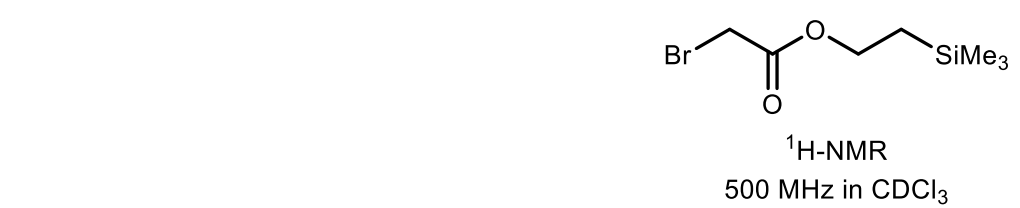

$500 \mathrm{MHz}$ in $\mathrm{CDCl}_{3}$
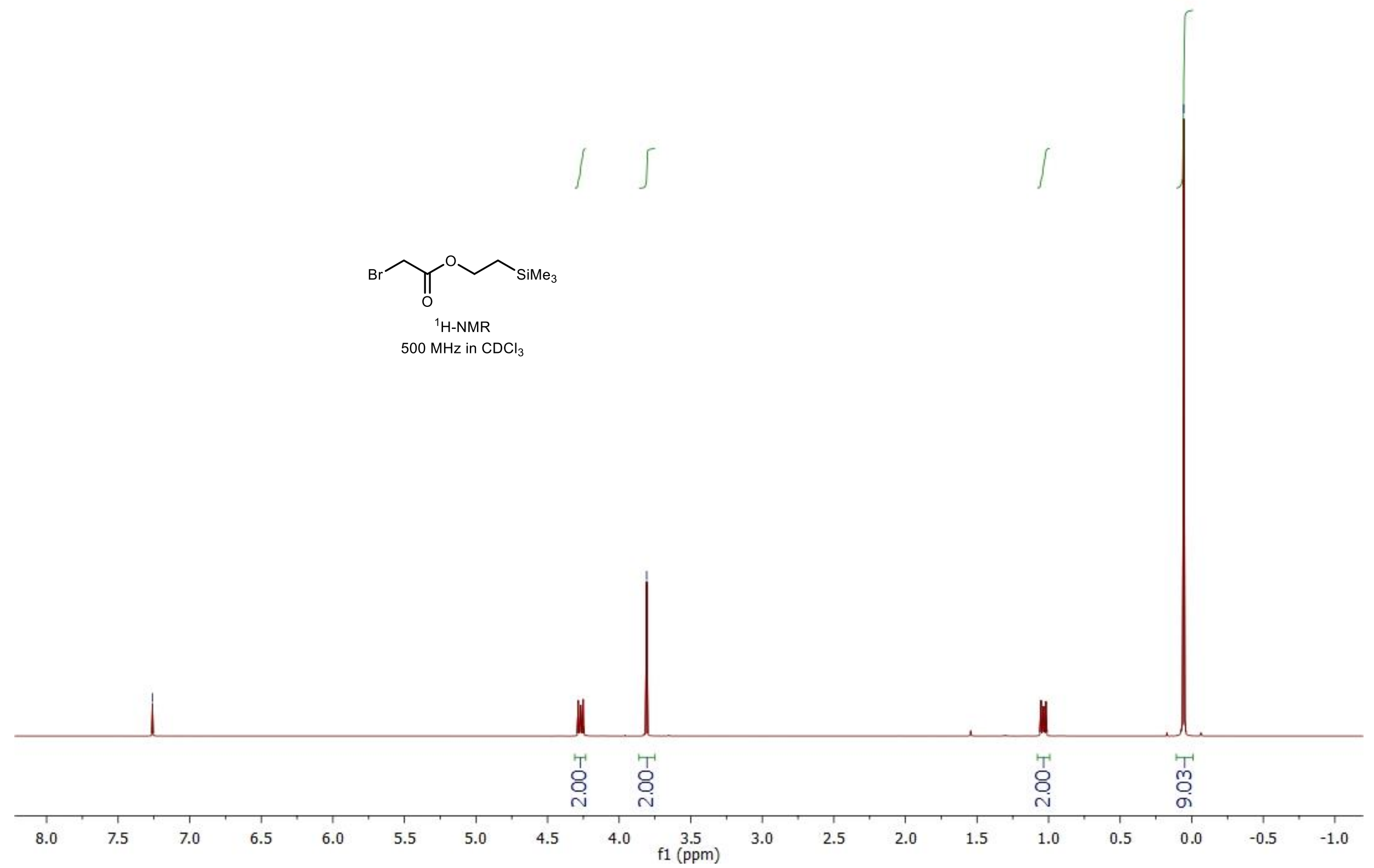

$68 / 77$ 
${ }^{13} \mathrm{C}-\mathrm{NMR}$ of compound 35

$$
\text { 告 }
$$

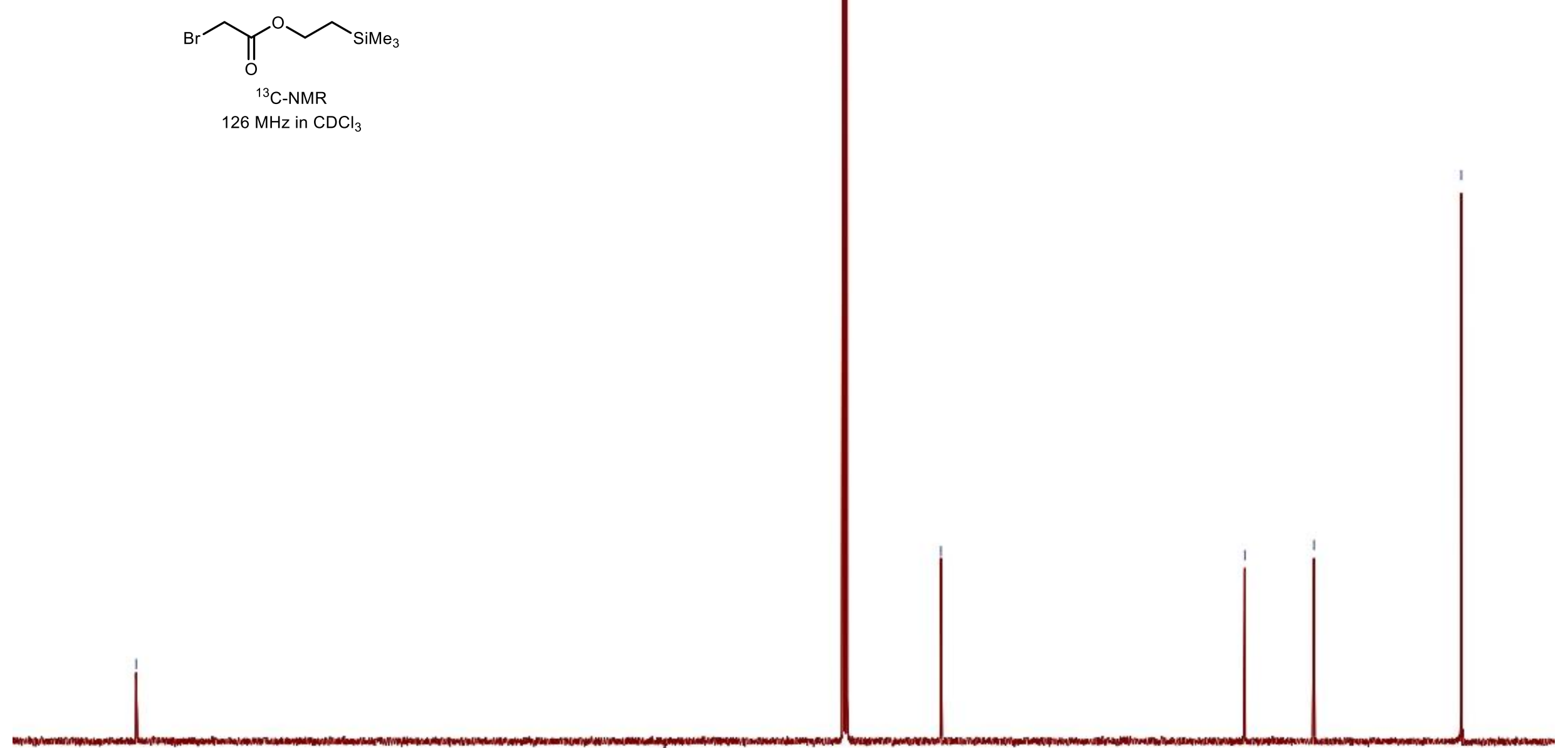

$126 \mathrm{MHz}$ in $\mathrm{CDCl}_{3}$

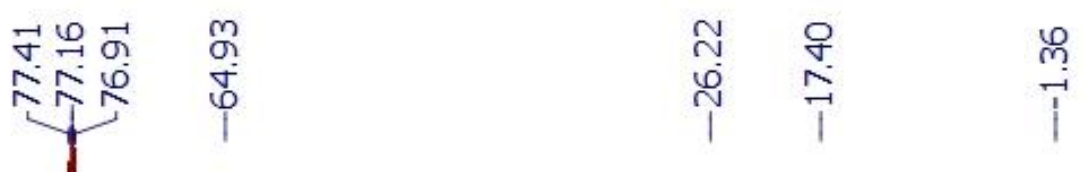

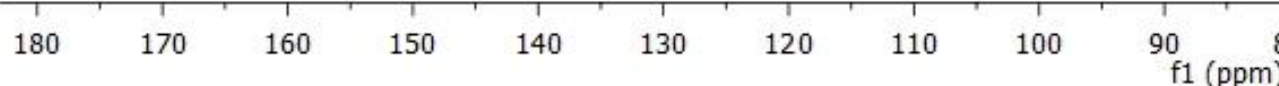

69 / 77 


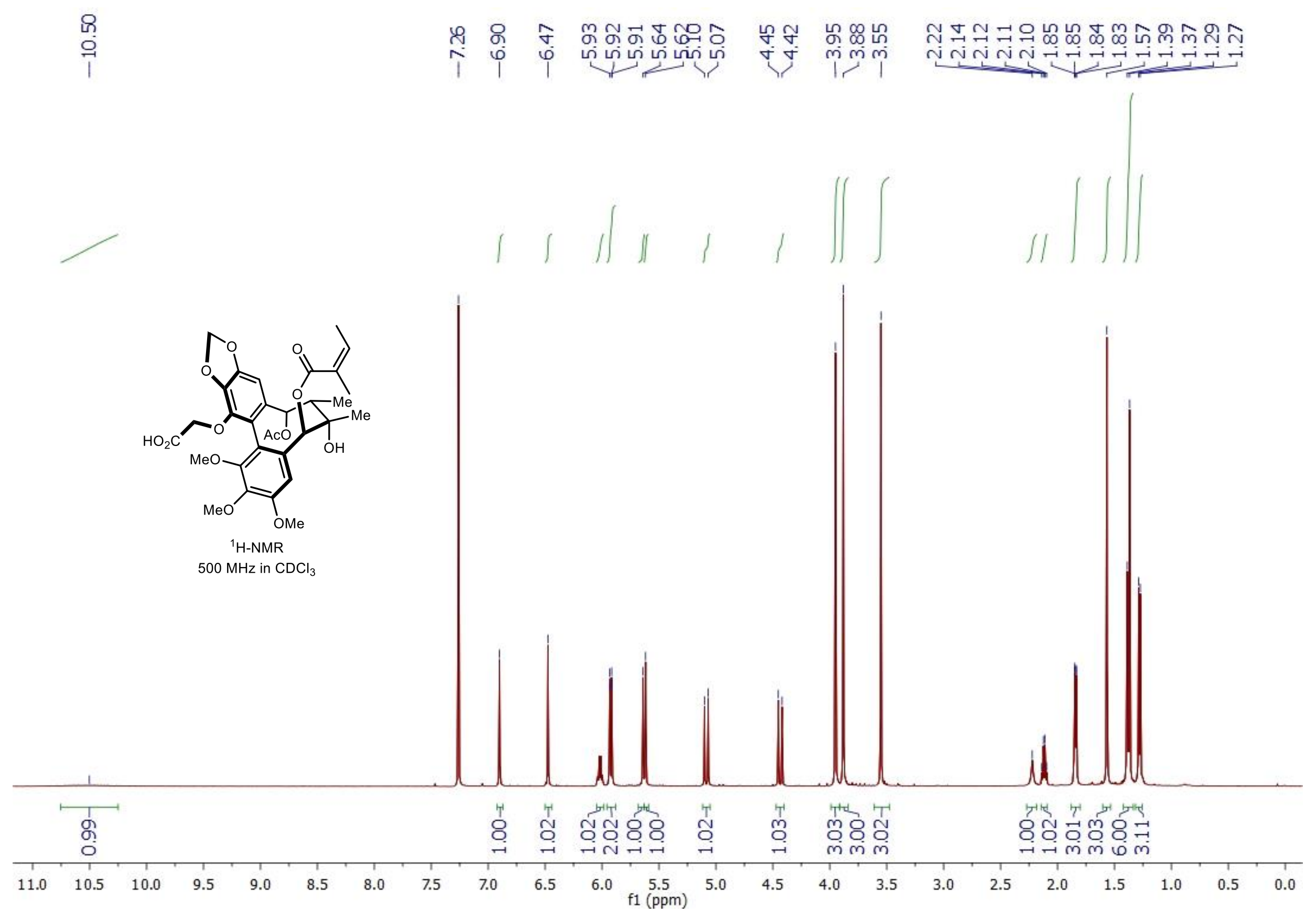




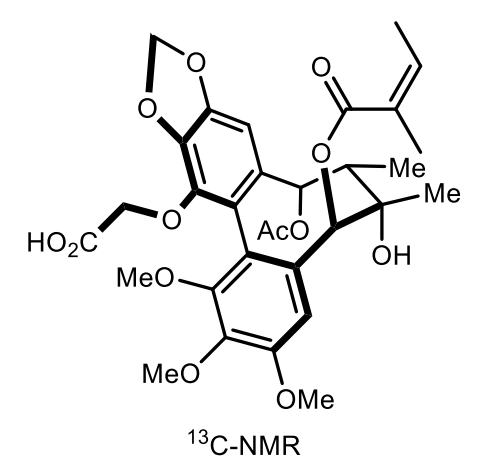

$126 \mathrm{MHz}$ in $\mathrm{CDCl}_{3}$ 


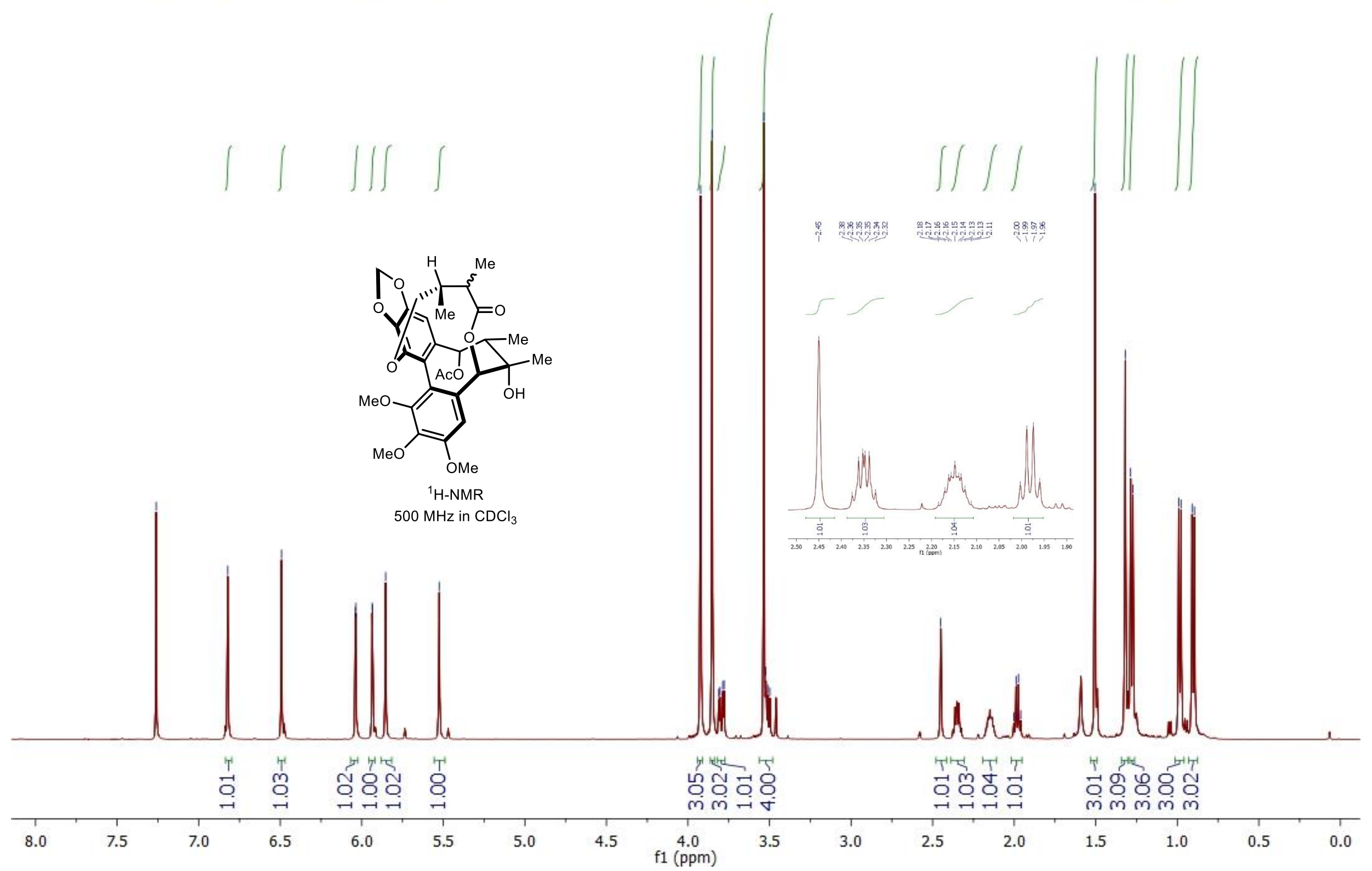



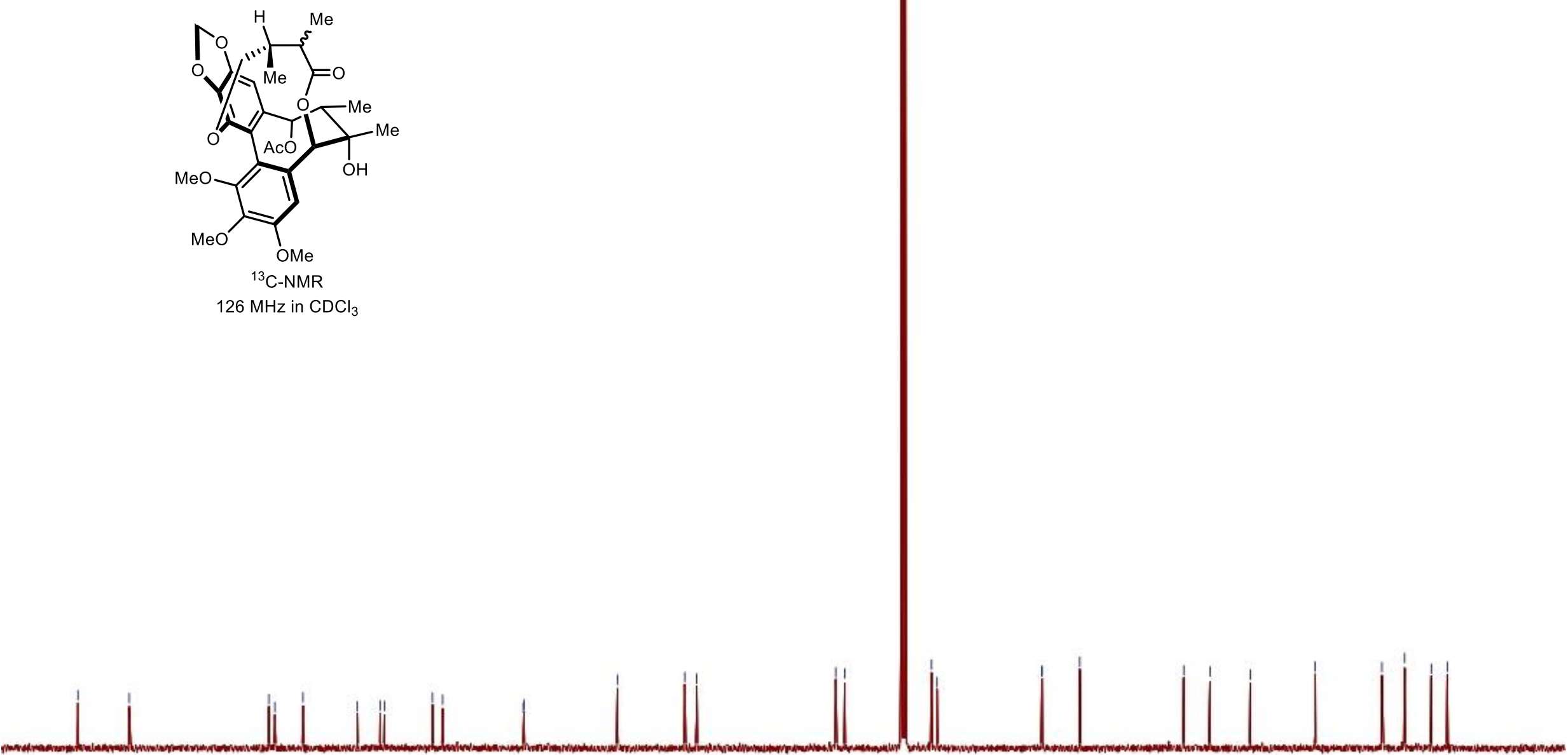


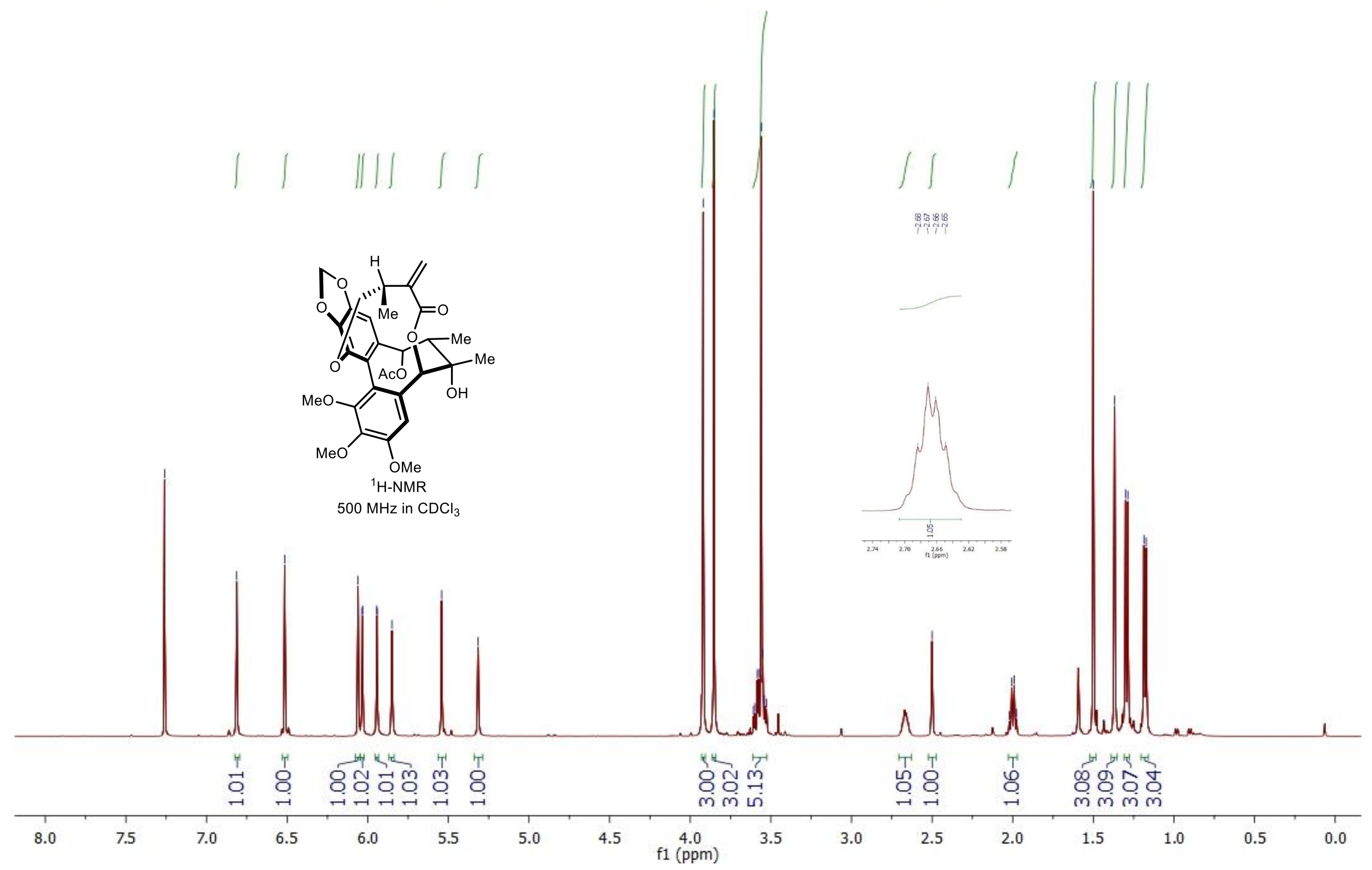



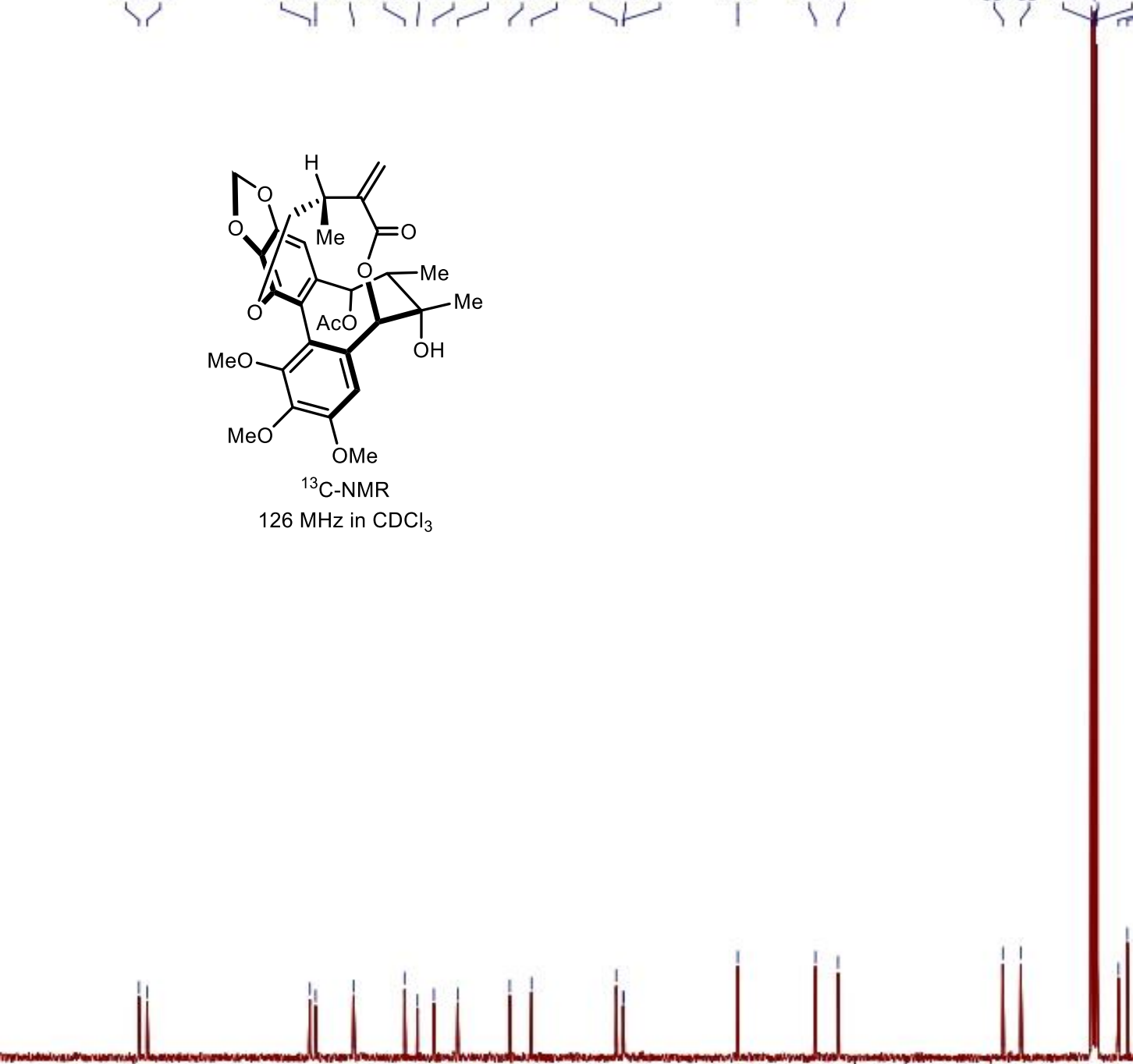


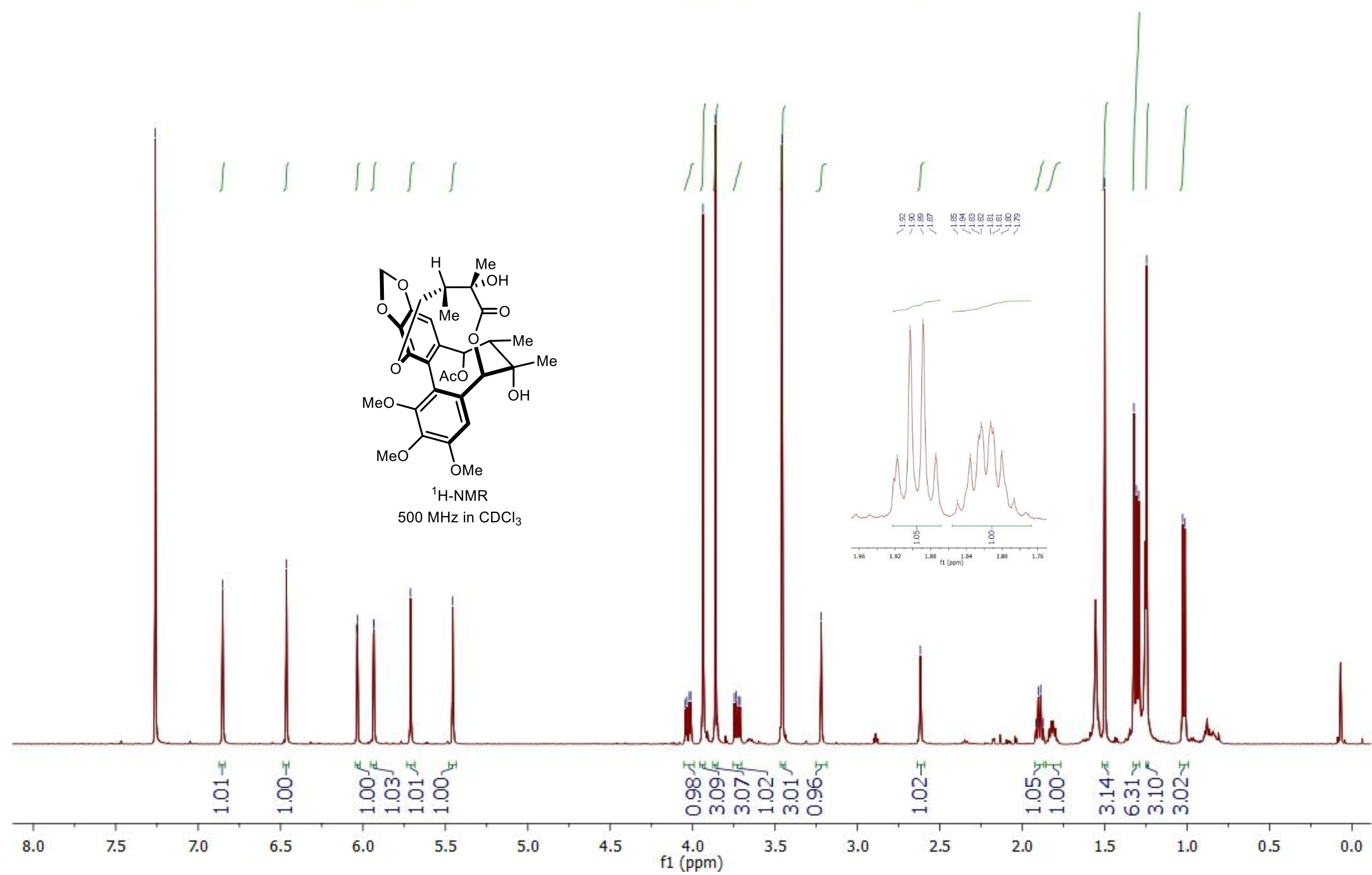




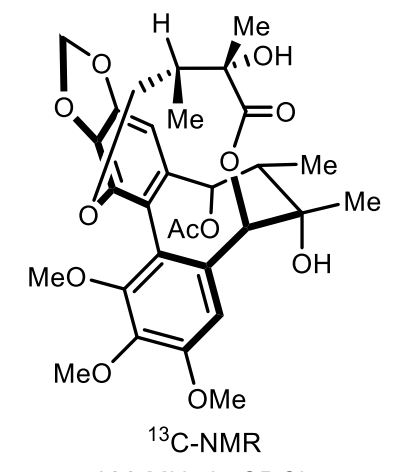

$126 \mathrm{MHz}$ in $\mathrm{CDCl}_{3}$

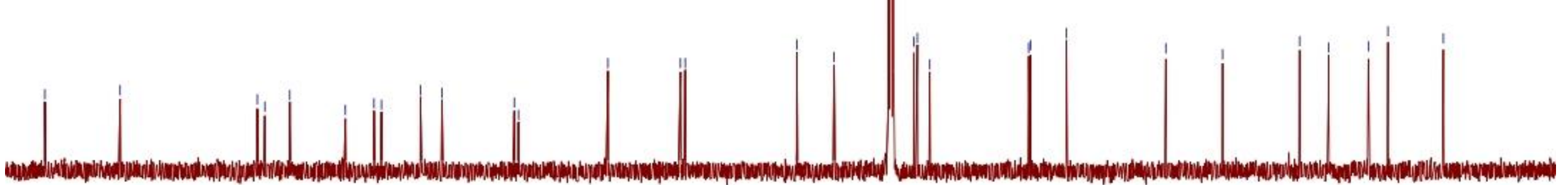

